\title{
An Improved Approach to Fracture Toughness Assessment of Brittle Coating on Ductile Substrate Systems under Indentation
}

\author{
by \\ Natalia V. Demidova, \\ B.Sc. (Honors), M.Sc. (Honors) \\ A thesis submitted to the Faculty of Graduate Studies and Research \\ in partial fulfillment of \\ the requirements for the degree of \\ Doctor of Philosophy \\ in
}

Mechanical Engineering

Ottawa-Carleton Institute for Mechanical and Aerospace Engineering

Department of Mechanical and Aerospace Engineering

Carleton University

Ottawa, Ontario

(C) Copyright,

2010, Natalia Demidova 


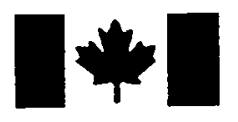

\author{
Library and Archives \\ Canada \\ Published Heritage \\ Branch \\ 395 Wellington Street \\ Ottawa ON K1A ON4 \\ Canada
}

Bibliotheque et

Archives Canada

Direction du

Patrimoine de l'édition

395 , rue Wellington

Ottawa ON K1A ON4

Canada
Your file Votre référence

ISBN: 978-0-494-70546-9

Our file Notre reférence

ISBN: 978-0-494-70546-9
NOTICE:

The author has granted a nonexclusive license allowing Library and Archives Canada to reproduce, publish, archive, preserve, conserve, communicate to the public by telecommunication or on the Internet, loan, distribute and sell theses worldwide, for commercial or noncommercial purposes, in microform, paper, electronic and/or any other formats.

The author retains copyright ownership and moral rights in this thesis. Neither the thesis nor substantial extracts from it may be printed or otherwise reproduced without the author's permission.
AVIS:

L'auteur a accordé une licence non exclusive permettant à la Bibliothèque et Archives Canada de reproduire, publier, archiver, sauvegarder, conserver, transmettre au public par télécommunication ou par l'Internet, prêter, distribuer et vendre des thèses partout dans le monde, à des fins commerciales ou autres, sur support microforme, papier, électronique et/ou autres formats.

L'auteur conserve la propriété du droit d'auteur et des droits moraux qui protège cette thèse. Ni la thèse ni des extraits substantiels de celle-ci ne doivent être imprimés ou autrement reproduits sans son autorisation.
In compliance with the Canadian Privacy Act some supporting forms may have been removed from this thesis.

While these forms may be included in the document page count, their removal does not represent any loss of content from the thesis.
Conformément à la loi canadienne sur la protection de la vie privée, quelques formulaires secondaires ont été enlevés de cette thèse.

Bien que ces formulaires aient inclus dans la pagination, il n'y aura aucun contenu manquant.

\section{Canadä}




\section{Abstract}

Fracture toughness is an important material property that determines the structural integrity of a component with pre-existing or service-generated flaws. In the present research, an indentation-based method and the associated fracture mechanics model are proposed for fracture toughness assessment of brittle coating/ductile substrate systems.

The proposed models consider well-developed radial/median cracks generated under sharp indentation, despite that the crack formation process may have gone through crack initiation and propagation phases. For generality, the geometry of a well-developed crack is assumed to be semi-elliptical in shape. The driving force of the crack is considered to stem from the residual plastic zone expansion under the indenter, as well as the far-field Boussinesq (elastic) stress. Three well-defined configurations are studied. For the first configuration, a crack with a depth of less than $7 \%$ of the coating thickness is considered. In this case, the problem is treated as the one for the monolithic material with the coating material properties. For the second configuration, a crack that runs deeper than $7 \%$ of the coating thickness but is still within the coating layer is analyzed. In this case, the composite hardness is introduced into the analysis to account for the influence of the substrate material properties; and furthermore, an interface correction factor is proposed to take into account the presence of the coating/substrate interface and its influence on the stress intensity factor of the well-developed elliptical cracks. For the third configuration, a crack penetrating into the substrate is considered. In this case, based on the condition of deformation compatibility across the coating/substrate interface, the bulk modulus for the coating/substrate system is introduced into the analysis. 
A series of indentation tests are conducted on a $\mathrm{WC} / 10 \mathrm{Co} / 4 \mathrm{Cr}$ coating/1080 low carbon steel substrate specimen, which is a brittle coating on a ductile substrate system, in order to obtain the required information for validating the proposed fracture toughness model. It is found that radial/median cracks are generated beneath the indenter within the coating layer in an elliptical shape and stopped at the interface under the indentation loads being used in the present experiments. Optical microscopy is employed to examine the surface (radial) crack lengths and the focused ion beam (FIB) technique is applied to dissecting the coating/substrate specimen and thereby allows for measuring the crack penetration depth; thus the full crack profile is determined. These tests have also verified the non-linear relationship between $c^{3 / 2}$ (where $c$ is a radial crack length) and the applied indentation load $P$, which is exhibited in the proposed fracture toughness model.

The fracture toughness of the tested $\mathrm{WC} / 10 \mathrm{Co} / 4 \mathrm{Cr}$ coating $/ 1080$ steel substrate system is determined in terms of the proposed fracture toughness model, utilizing the experimental results. The fracture toughness value for this coating/substrate system is then compared to the known fracture toughness value of a similar brittle coating/ductile substrate system (WC/12Co coating/1020 low carbon steel substrate) and is found to be comparable. The proposed fracture toughness model best describes the experimental observation of cracking/fracture behavior of brittle coating/ductile substrate systems under indentation, compared with other existing models, which can be utilized to assess the fracture toughness of these coating/substrate systems. The developed approach makes a significant improvement in the existing fracture mechanics methods for fracture toughness assessment of brittle coating/ductile substrate systems. 
In the loving memory of my grandmother, Albina Grigorevna Kostenko, who showed me the path to success through hard work, patience, humility and dedication. 


\section{Acknowledgements}

I owe my sincere gratitude to my supervisors, Prof. Rong Liu (Carleton University, Canada) and Dr. Xijia Wu (National Research Council, Canada) for their constant support and valuable suggestions. Thank you for being there for me when I needed your advice or recommendations.

I would like to express my great appreciation to my future husband, Kyriacos Miltiadous, for his continuous support and patience during the write-up phase of my Ph.D. thesis. I would also like to thank my parents, Marina and Sergey, for having faith in me and providing me with their guidance throughout the years.

I would like to thank my colleagues from the Canadian Nuclear Safety Commission, Canada. Special thanks to Raoul Awad, Director of the Operational Engineering Assessment Division (OEAD), for providing me with time to focus on my $\mathrm{Ph} . \mathrm{D}$. thesis write-up. I am also grateful for having constructive discussions with Dr. Nicolas Christodoulou and Glen McDougall from the OEAD.

I thank the staff of the Mechanical and Aerospace Engineering at Carleton University for all the help that they have provided me with throughout my studies at Carleton University. 


\section{Table of Contents}

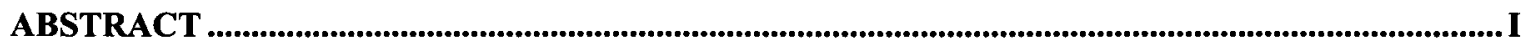

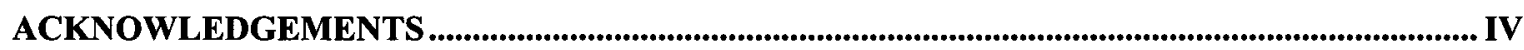

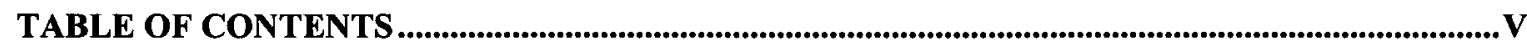

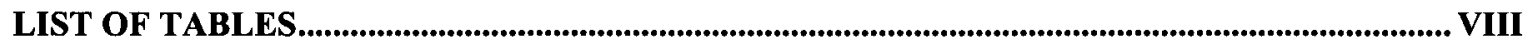

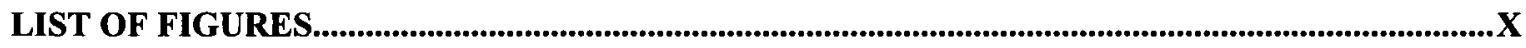

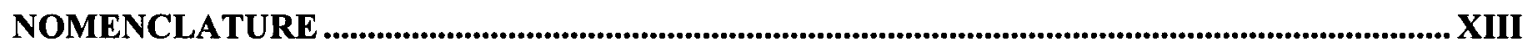

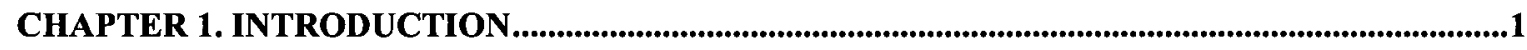

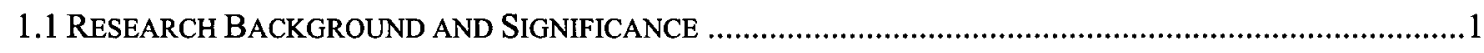

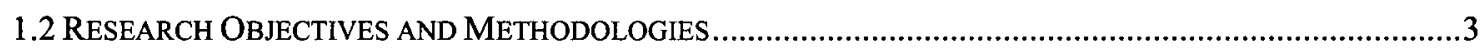

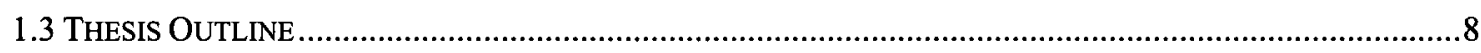

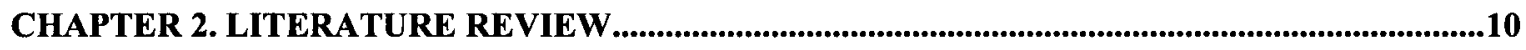

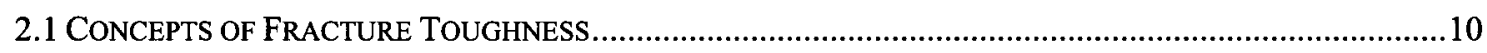

2.2 AN OVERVIEW of Fracture TOUGHNESS MEASUREMENTS For BRITTLE COATngs ........................12

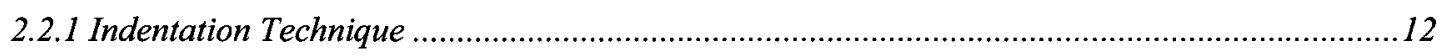

2.2.1.1 Indentation Fracture Toughness Evaluation for Median Cracks......................................................... 12

2.2.1.2 Indentation Fracture Toughness Evaluation for Palmqvist Cracks .................................................. 23

2.2.1.3 Indentation Fracture Toughness Evaluation for Ring Cracks ........................................................... 26

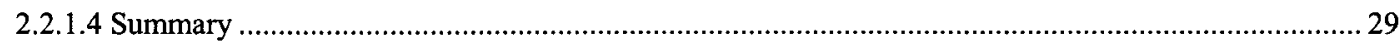

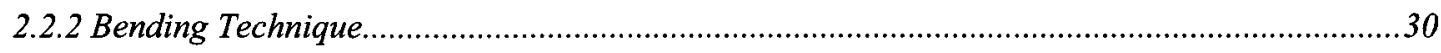

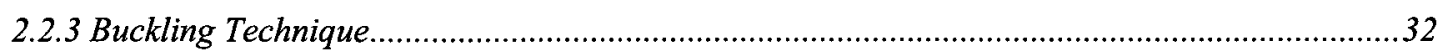

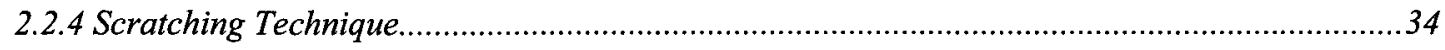

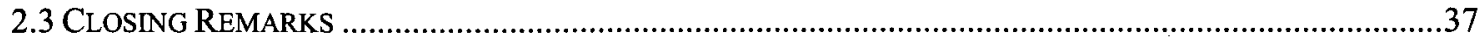




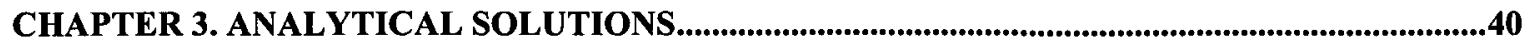

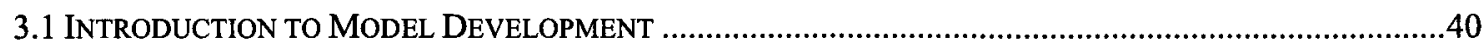

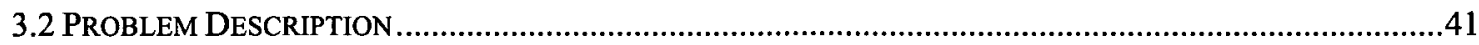

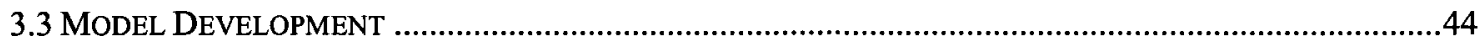

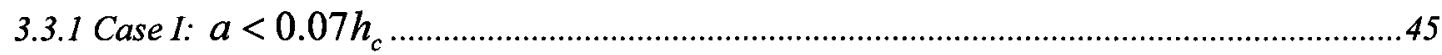

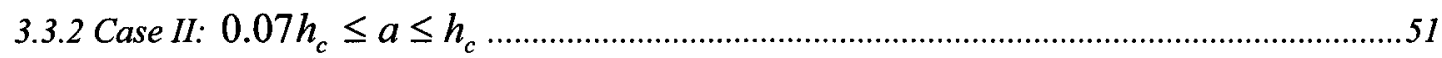

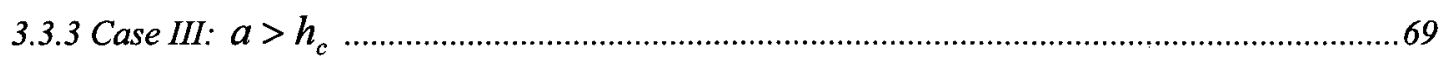

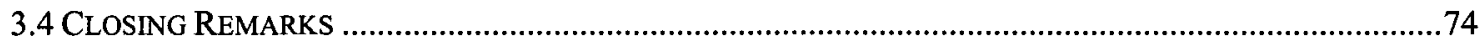

CHAPTER 4. EXPERIMENTAL DETAILS.............................................................................................76

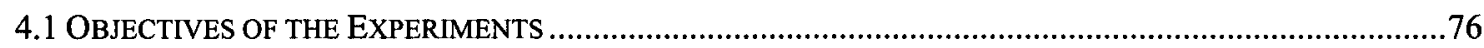

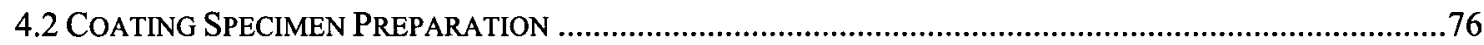

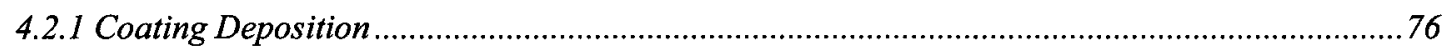

4.2.2 Specimens for Coating Thickness Measurement ..............................................................77

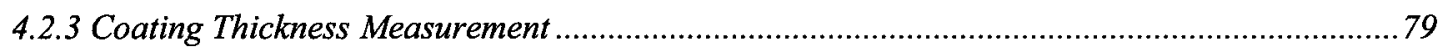

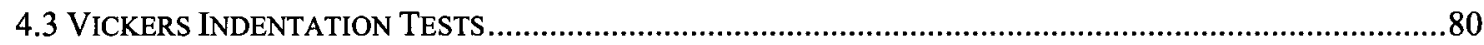

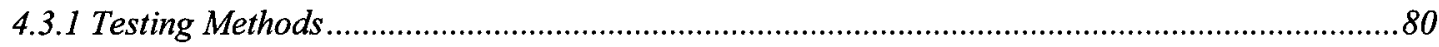

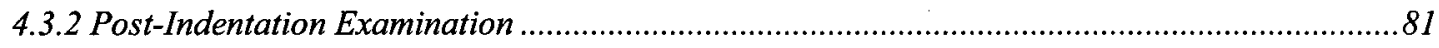

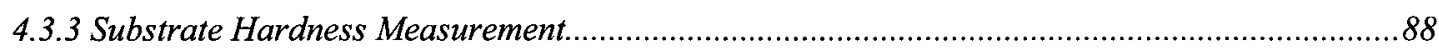

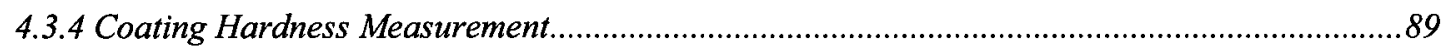

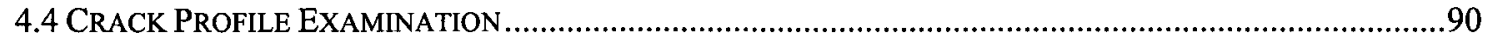

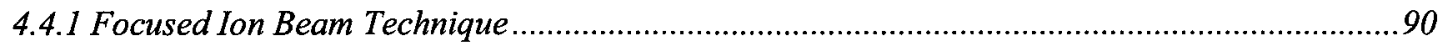

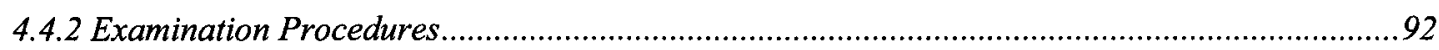

4.4.3 Post-Indentation Coating Surface Examination .................................................................93

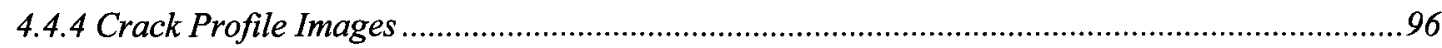

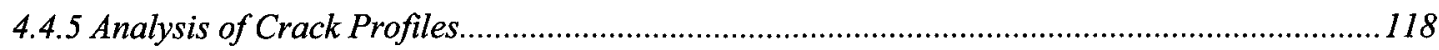

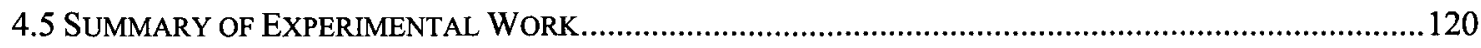




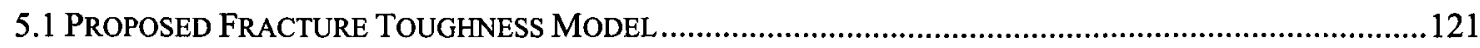

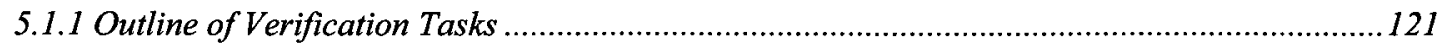

5.1.2 Relevant Considerations for the Proposed Model ...........................................................122

5.1 .3 Evaluation of Key Parameters in the Proposed Model .......................................................125

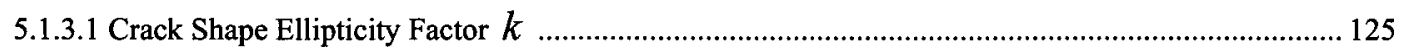

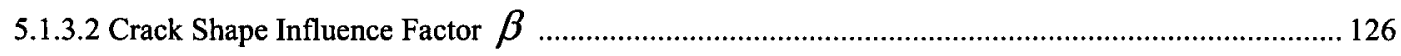

5.1.3.3 Residual Factor $\chi_{r}$ Corresponding to Residual Stress Component .............................................. 129

5.1.4 Determination of Fracture Toughness with the Proposed Model .........................................131

5.1.5 Response Behaviour of Crack Length to Indentation Load .................................................134

5.2 COMPARISON BETWEen THE Proposed MODEL AND OTHER EXISTING MOdELS................................136

5.2.1 Smith-Scattergood Fracture Toughness Model...................................................................136

5.2.2 Lawn et al. Fracture Toughness Model ........................................................................... 140

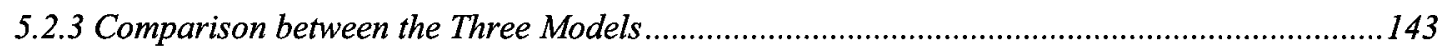

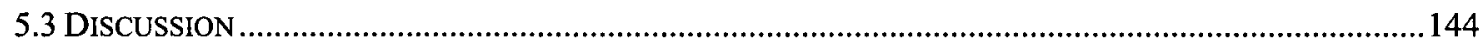

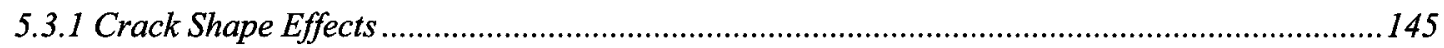

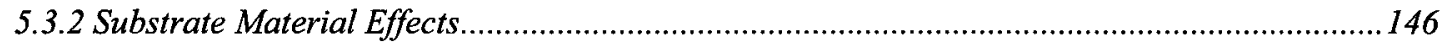

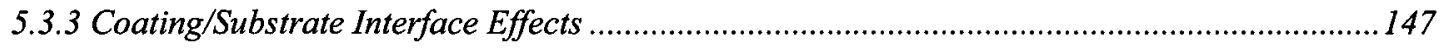

5.3.4 Prediction of Fracture Toughness and Fracture Behaviour ...............................................149

5.3.5 Implications for the Coating System Design ................................................................... 151

CHAPTER 6. CONCLUSIONS AND FUTURE WORK ….................................................................154

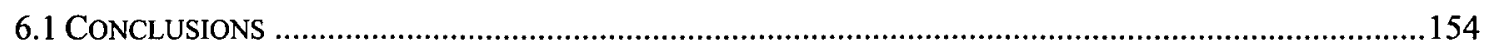

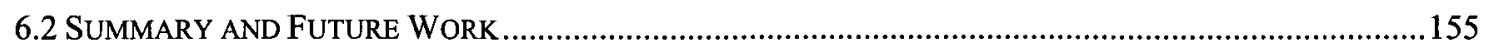

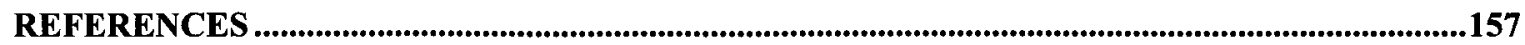




\section{List of Tables}

Table 3-1. A boundary correction factor $F_{I}$ for an elliptical crack near a bi-material interface at point $A$

Table 3-2. Interface correction factor $Y_{I}=F_{I} \cdot \sqrt{Q}$ at point $A$. 56

Table 3-3. Proposed interface correction factor $Y_{I}$ for an elliptical crack near a bi-material interface at point $A$

Table 3-4. A summary of $F_{I}$ values corresponding to the cases $\mu_{s} / \mu_{c}=0.5$ and $\mu_{s} / \mu_{c}=2$ at $h / a=1.2, a / c=0.5$

Table 3-5. A summary of $Y_{I n}$ values corresponding to the cases $\mu_{s} / \mu_{c}=0.5$ and $\mu_{s} / \mu_{c}=2$ at $h / a=1.2, a / c=0.5$

Table 3-6. Percent error (\%) between $Y_{I n}$ and $F_{I}$ at $\mu_{s} / \mu_{c}=0.5, h / a=1.2, a / c=0.5$;

and at $\mu_{s} / \mu_{c}=2, h / a=1.2, a / c=0.5$.

Table 4-1. Average radial crack lengths $c(\mu \mathrm{m})$ at corresponding loads $P(\mathrm{~N})$. 87

Table 4-2. Average values of indentation diagonals $l(\mu \mathrm{m})$ in the $\mathrm{WC} / 10 \mathrm{Co} / 4 \mathrm{Cr}$ coating. 88

Table 4-3. Average diagonals $l(\mathrm{~mm})$ of indentation marks on the 1080 low carbon steel substrate and the resulting hardness values $H_{s}(\mathrm{GPa})$ 89

Table 4-4. A summary of hardness values $H_{c}$ (HV) of the WC/10Co/4Cr coating...... 90

Table 4-5. A summary of the observed cracks.

Table 5-1. A summary of the crack shape ellipticity factor $k$ values. 125

Table 5-2. A summary of the crack shape influence factor $\beta$ values. 127 
Table 5-3. A summary of the material parameters of the $\mathrm{WC} / 10 \mathrm{Co} / 4 \mathrm{Cr}$ coating $/ 1080$ steel

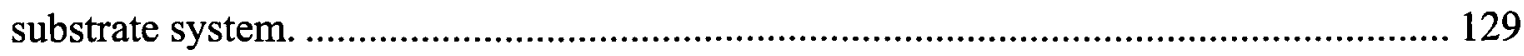

Table 5-4. A summary of the composite hardness $H_{m}$ values..................................... 130

Table 5-5. A summary of the residual factor $\chi_{r}$ values............................................... 131

Table 5-6. Fracture toughness values based on the proposed model............................... 132

Table 5-7. Ratios of hardness to Young's modulus of the two similar coating/substrate

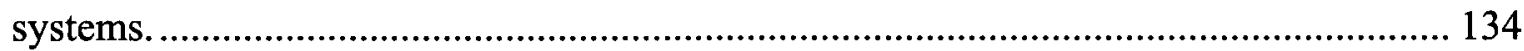

Table 5-8. A summary of $f_{\text {res }}$ values corresponding to $k$ values................................. 137

Table 5-9. Fracture toughness values based on Smith-Scattergood model. ................... 138

Table 5-10. Fracture toughness values based on Lawn et al. model. ............................. 141 


\section{List of Figures}

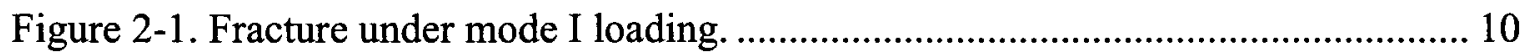

Figure 2-2. A stress field near a crack tip in a linear-elastic material................................ 11

Figure 2-3. Median (half-penny) crack geometry under Vickers indentation. .................. 14

Figure 2-4. Palmqvist crack geometry under Vickers indentation.................................. 24

Figure 2-5. Schematic of a load-displacement curve showing a step with energy release

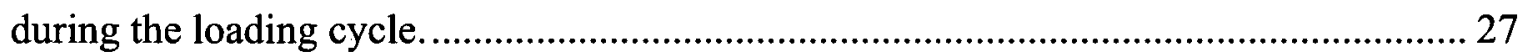

Figure 2-6. Schematic diagram of the pre-crack introduced with a bending method....... 30

Figure 2-7. Schematic of the three-point bending test for the freestanding film with a

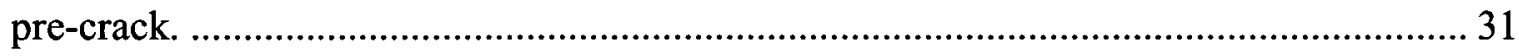

Figure 2-8. Schematic of the buckling test with simple support ends............................. 33

Figure 2-9. Schematic of the stylus drawn along the coated specimen surface................ 35

Figure 3-1 a. Schematic of a radial/median crack with the depth of $0.07 h_{c}$ or less in a

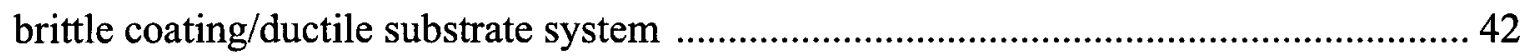

Figure 3-1 b. Schematic of an elastic component during full loading .............................. 43

Figure 3-1 c. Schematic of a residual component after complete indenter removal ........ 44

Figure 3-2. Schematic of a radial/median crack penetrating into the substrate at full

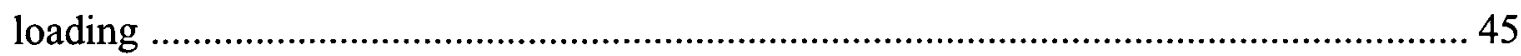

Figure 3-3. Notation for the weight function regarding an embedded elliptical crack .... 50

Figure 3-4. An elliptical crack perpendicular to a bi-material interface subjected to remote tensile loading 54

Figure 3-5. Comparison between the proposed interface correction factor $Y_{I}$ predicted with Eq. (3-24) to those values calculated with Eq. (3-23)............................................... 60 
Figure 3-6. Screenshot of xyExtract software with the graph from Noda et al.

Figure 3-7. Comparison of the normalized angular interface correction factor $Y_{I a}$ to $F_{I}$ of

Noda et al. 66

Figure 4-1. SEM images of the cross-section of the WC/10Co/4Cr coating/1080 low

carbon steel substrate specimen: (a) before polishing and (b) after polishing. 80

Figure 4-2. Optical images of the indented $\mathrm{WC} / 10 \mathrm{Co} / 4 \mathrm{Cr}$ coating surfaces with the radial crack length and indentation mark diagonal measurements. 86

Figure 4-3. Focused Ion Beam system set-up 91

Figure 4-4. Focused Ion Beam system sputtering process 92

Figure 4-5. Typical radial/median cracks sectioned at four locations along their lengths in the plane normal to the coating surface. 93

Figure 4-6. Top views of the indented $\mathrm{WC} / 10 \mathrm{Co} / 4 \mathrm{Cr}$ coating surface at all indentation loads. .95

Figure 4-7. Correlation between radial, median, lateral and edge cracks. 97

Figure 4-8. Cross-sectional views of the $\mathrm{WC} / 10 \mathrm{Co} / 4 \mathrm{Cr}$ coating/1080 steel substrate system at $P=196 \mathrm{~N}$.

Figure 4-9. Cross-sectional views of the $\mathrm{WC} / 10 \mathrm{Co} / 4 \mathrm{Cr}$ coating/1080 steel substrate system at $P=294 \mathrm{~N}$. 105

Figure 4-10. Cross-sectional views of the $\mathrm{WC} / 10 \mathrm{Co} / 4 \mathrm{Cr}$ coating/1080 steel substrate system at $P=392 \mathrm{~N}$ 109

Figure 4-11. Cross-sectional views of the $\mathrm{WC} / 10 \mathrm{Co} / 4 \mathrm{Cr}$ coating/1080 steel substrate system at $P=490 \mathrm{~N}$. 
Figure 4-12. Cross-sectional views of the $\mathrm{WC} / 10 \mathrm{Co} / 4 \mathrm{Cr}$ coating/1080 steel substrate system at $P=720 \mathrm{~N}$.

Figure 5-1. Crack shape influence factor $\beta$ vs. the crack shape ellipticity factor $k \ldots . .128$

Figure 5-2. Fracture toughness values based on the proposed model............................ 132

Figure 5-3. Non-linear behavior of radial crack length $c^{3 / 2}$ vs. indentation load $P \ldots . .135$

Figure 5-4. Fracture toughness values based on Smith-Scattergood model.................... 139

Figure 5-5. Fracture toughness values based on Lawn et al. model............................. 141

Figure 5-6. Comparison of the fracture toughness values predicted with the proposed model, Smith-Scattergood model and Lawn et al. model. 143 


\section{Nomenclature}

$a$

$a_{p c}$

$a_{s c r}$

$A_{c}$

$A_{s}$

$A_{\text {total }}$

$b$

$b_{s p}$

$c$

$c_{\exp }$

$c_{m}$

$C_{R}$

$d$

$d_{\text {ind }}$

$D(\alpha, \gamma) \quad$ function of Dundur's parameters

$e$

E

$E(k)$

$E_{b}$

crack length at scratching

area of plastic zone in coating

area of plastic zone in substrate

total area of plastic zone

radius of spherical plastic zone

crack spacing

crack length

experimental radial crack length

depth of indenter penetration

shortening of beam

Young's modulus

elliptical integral of second kind crack depth (corresponds to minor ellipse axis)

length of pre-existing crack at bending

median (half-penny) crack length

length of ring-like through-thickness crack in film plane

depth of Vickers indenter penetration into substrate

Young's modulus of bulk brittle material 


\begin{tabular}{|c|c|}
\hline$E_{c}$ & Young's modulus of coating \\
\hline$E_{m}$ & Young's modulus of coating/substrate system \\
\hline$E_{s}$ & Young's modulus of substrate \\
\hline$f$ & function of $a / W$ \\
\hline$f_{i j}^{(I)}$ & angular function for mode I loading \\
\hline$f_{c}$ & volume fraction of plastic zone under pressure in coating \\
\hline$f_{s}$ & volume fraction of plastic zone under pressure in substrate \\
\hline$f_{s c r}\left(a_{s c r}, b_{s p}\right)$ & non-dimensional function at scratching test \\
\hline$f_{\text {res }}$ & shape correction factor \\
\hline$F_{I}$ & dimensionless stress intensity factor normalized by $\sigma_{\infty} \sqrt{\pi a}$ \\
\hline$F_{\text {res }}$ & shape correction factor \\
\hline$g$ & Palmqvist crack length \\
\hline$g_{\text {res }}$ & constant $(\approx 1.22)$ \\
\hline$G_{c}(\varphi)$ & angular function \\
\hline$G_{c r i t}$ & critical strain energy release rate \\
\hline$h$ & distance between crack and bi-material interface \\
\hline$h_{c}$ & coating thickness \\
\hline$h_{s}$ & substrate thickness \\
\hline$H$ & Hardness \\
\hline$H_{b}$ & Vickers hardness of bulk brittle material \\
\hline
\end{tabular}




\begin{tabular}{|c|c|}
\hline$H_{c}$ & coating hardness \\
\hline$H_{m}$ & composite hardness \\
\hline$H_{s}$ & substrate hardness \\
\hline$k$ & crack shape ellipticity factor \\
\hline$k_{c}$ & bulk modulus of coating \\
\hline$K$ & stress intensity factor \\
\hline$K_{b}(t)$ & elliptical integral used in buckling method \\
\hline$K_{c}$ & fracture toughness \\
\hline$K_{e}$ & stress intensity factor corresponding to elastic component \\
\hline$K_{I}$ & stress intensity factor corresponding to mode I loading \\
\hline$K_{I, \infty}$ & $\begin{array}{l}\text { stress intensity factor for embedded elliptical crack in infinite } \\
\text { medium under tensile loading }\end{array}$ \\
\hline$K_{m}$ & bulk modulus of coating/substrate system \\
\hline$K_{r}$ & stress intensity factor corresponding to plastic component \\
\hline$l$ & half-diagonal of indenter \\
\hline$L(k)$ & elliptical function \\
\hline$L_{\text {beam }}$ & length of beam \\
\hline$M(x, y)$ & weight function \\
\hline$M_{b}(t)$ & elliptical integral used in buckling method \\
\hline$m$ & constant $(\approx 1 / 2)$ \\
\hline$n$ & number of collocation points \\
\hline
\end{tabular}




\begin{tabular}{|c|c|}
\hline$p_{b}$ & hydrostatic compression induced by elastic matrix \\
\hline$p_{c}$ & average hydrostatic pressure in coating \\
\hline$p_{s}$ & average hydrostatic pressure in substrate \\
\hline$P$ & indentation load \\
\hline$P_{b}$ & bending load \\
\hline$P^{\prime}$ & point on elliptical crack front \\
\hline$P^{\prime \prime}$ & load point \\
\hline$p_{a v}$ & average pressure distributed over internal surface \\
\hline$P_{c}$ & load at fracture during bending \\
\hline$P_{r}$ & force exuded by plastic field approximated as point \\
\hline$Q$ & crack shape factor \\
\hline$r$ & length in polar coordinates \\
\hline$R$ & distance from crack tip \\
\hline$R_{b}$ & radius of curvature \\
\hline$s$ & shortest distance between load point and crack boundary \\
\hline$S$ & span between two supporting positions \\
\hline$S^{\prime}$ & crack front boundary \\
\hline$t$ & sinusoidal function \\
\hline$U$ & elastic strain energy difference before and after cracking \\
\hline$V$ & volume of plastic zone \\
\hline$W$ & coating width \\
\hline
\end{tabular}




\begin{tabular}{|c|c|}
\hline$(\mathrm{x}, \mathrm{y}, \mathrm{z})$ & Cartesian coordinate system \\
\hline $\mathrm{X}$ & contraction ratio \\
\hline$Y$ & interface boundary factor \\
\hline$Y_{I}$ & interface correction factor for mode I load \\
\hline$Y_{I a}$ & angular interface correction factor \\
\hline$Y_{I n}$ & normalized angular interface correction factor \\
\hline$Z$ & crack shape factor \\
\hline$\alpha$ & Dundur's parameter \\
\hline$\beta$ & crack shape influence factor \\
\hline$\chi$ & coefficient of proportionality \\
\hline$\chi_{e}$ & calibration coefficient corresponding to elastic stress component \\
\hline$\chi_{r}$ & residual factor \\
\hline$\delta$ & empirical constant depending on indenter geometry \\
\hline$\delta V$ & volume of indentation impression \\
\hline$\varepsilon_{\text {bend }}$ & bending strain \\
\hline$\varepsilon^{c}$ & volumetric strain in coating \\
\hline$\varepsilon_{\text {crit }}$ & critical strain \\
\hline$\varepsilon^{s}$ & volumetric strain in substrate \\
\hline$\left(\varepsilon_{x}^{c}, \varepsilon_{y}^{c}, \varepsilon_{z}^{c}\right)$ & strain components associated with coating \\
\hline$\left(\varepsilon_{x}^{s}, \varepsilon_{y}^{s}, \varepsilon_{z}^{s}\right)$ & strain components associated with substrate \\
\hline$\phi$ & constraint factor $(\approx 3)$ \\
\hline
\end{tabular}




\begin{tabular}{ll}
$\gamma$ & Dundur's parameter \\
$\varphi$ & angle in polar coordinates \\
$\lambda$ & adjustable parameter \\
$\lambda_{\max }$ & best-fit parameter \\
$\mu$ & shear modulus \\
$\mu_{c}$ & shear modulus of coating \\
$\mu_{s}$ & shear modulus of substrate \\
$v_{c}$ & Poisson's ratio of coating \\
$v_{m}$ & Poisson's ratio of coating/substrate system \\
$v_{s}$ & Poisson's ratio of substrate \\
$\theta$ & angle around crack tip \\
$\rho_{i}(i=1 \ldots 4)$ & parameters controlled by shape of ellipse \\
$\sigma_{\infty}(r, \varphi)$ & stress distribution over crack plane \\
$\sigma_{a p p}$ & applied stress \\
$\sigma_{i j}^{(I)}$ & stress components for mode I loading \\
$\sigma_{y}^{\infty}$ & uniform tension in y-direction \\
$\left.\sigma_{z}^{c}, \sigma_{y}^{c}, \sigma_{z}^{c}\right)$ & stress components associated with coating \\
\hline
\end{tabular}


$\left(\sigma_{x}^{s}, \sigma_{y}^{s}, \sigma_{z}^{s}\right) \quad$ stress components associated with substrate

$\xi \quad$ parametric polar angle of ellipse

$\psi \quad$ indenter half- angle

$\Delta$

percent error

$\Theta$

bending angle

$\Phi(\varphi, k) \quad$ elliptical function 


\section{Chapter 1. Introduction}

\subsection{Research Background and Significance}

Coatings are extensively used as integral parts of engineering components to combat thermal, abrasive and environmental attacks such as wear, erosion, corrosion, machining, and grinding [1,2]. A hard outer layer confers enhanced load-bearing capacity, wear resistance, thermal and corrosion protection while a softer substrate provides stress redistribution and mitigates impact [3]. In order to increase the overall in-service life, improve performance and reliability, reduce friction/wear/fretting damage, and increase corrosion and thermal protection, thin brittle coatings (mostly ceramic) are often applied on tools, engines and machine components [2]. In medical devices and implants, brittle coatings provide longer in-service life, wear resistance, biocompatibility, and chemical durability [4].

In such applications, the integrity of protective coatings determines the useful life of the components since coating failure (spallation or cracking) usually either exposes the substrate to hostile environment or initiates cracks to propagate under fatigue loading. Therefore, the fracture toughness $K_{c}$ is a paramount material property concerning the design of engineering components with protective coatings.

The methodologies currently employed to analyze the fracture toughness of thin brittle coatings include indentation, bending, buckling, and scratching tests [5]. Bending, buckling and scratching tests require complex high-precision experimental procedures and a significant number of samples for testing. On the contrary, indentation methods 
provide the simplicity and convenience allowing for a straightforward experimental assessment of fracture toughness using a small number of samples.

Employing either Vickers, Knoop or Berkovich indenter, various indentation-based fracture mechanics models for bulk brittle materials have been developed over time, assuming the formation of well-developed median (half penny-shape) cracks in the coating/substrate systems. Many of these methods have proven to be effective for fracture toughness assessment of bulk brittle materials with various material properties [6-11]. However, the fracture toughness analysis becomes less straightforward when the same indentation methods are applied to assess the fracture toughness of brittle coating/ductile substrate systems. When applying the models initially developed for bulk brittle materials to thick brittle coatings $(>200 \mu \mathrm{m})$, the reliability of these engaged methods has been well documented [7], while attempts to apply similar methods to thin coatings $(<100 \mu \mathrm{m})$ result in at least $30 \sim 40 \%$ uncertainty [12]; thus providing unreliable results with respect to fracture toughness assessment. This could be attributed to a more complex underlying mechanism contributing to the fracture behavior in the bi-layer systems. For example, well-developed cracks generated from the indentation might be arrested at the interface, thus imminently departing from a perfect half-penny shape postulated for bulk brittle materials. Moreover, at high indentation loads, a well developed crack can also be affected by the presence of the coating/substrate interface and the material properties of the substrate. Obviously, these models originally developed for bulk brittle materials, are lacking the consideration of aforementioned factors that may play a crucial role in affecting the fracture toughness of brittle coatings on ductile substrates. Hence, their applications to the coating/substrate 
systems are limited. Therefore, for the case where indentation depth exceeds $7 \%$ of the coating thickness, there are serious concerns regarding the influence of the substrate and applicability of the prior existing models [5]. This research is motivated by the necessity to have an accessible indentation-based model that would be developed specifically for the bi-layer systems and could be easily employed for fracture toughness assessment of brittle coatings of various thicknesses on ductile substrates.

\subsection{Research Objectives and Methodologies}

The primary objective of this research is to develop a universal indentation approach based on the principles of linear elastic-plastic indentation fracture mechanics, which can be utilized to evaluate the fracture toughness of brittle coating/ductile substrate systems subjected to a sharp contact load. Theory and analysis focus on the well-developed cracks formed under sharp indentation. Due to the nature of brittle coatings, crack nucleation would occur immediately upon loading and its further propagation would rapidly reach a stable size. Therefore, a well-developed crack means that a crack has propagated to a length that is well beyond the influence of the near field around the indenter and the associated plastic zone. Furthermore, for generality, the radial/median cracks generated in the brittle coating/ductile substrate system under indentation are assumed to be semi-elliptical in shape due to the presence of the interface.

The formulation of the fracture mechanics model follows the general scheme originally proposed by Lawn et al. [11] and further refined by other researchers [13-17], where the stress field due to the crack-driving force under an indentation load is treated as elastic-plastic in nature. The elastic-plastic field at the full load is represented as a superposition of a residual plastic stress field and an elastic field acting outside of the 
plastic zone generated under the contact of the indenter with the specimen. The elastic field reaches its maximum at the full loading and diminishes completely during unloading. The plastic component, by nature, reaches its maximum at full loading but persists throughout the unloading phase due to the irreversible expansion of the plastic zone developed under the full load of indentation, in accordance with the scheme proposed by Lawn et al. [11]. Thus, by superposition, the total stress intensity factor of the well-developed crack geometry is a superposition of the elastic and residual stress intensity factors which corresponds to the elastic and plastic stress fields respectively.

The elastic component that operates outside of the plastic zone can be approximated with a classic Boussinesq solution for a point force acting on a semi-infinite plane. In the realm of the well-developed cracks, i.e., the cracks having a much larger size than the size of the plastic zone under an indenter, the residual field can be treated as an outward crack driving force which appears as a push force of the plastically expanded material on the surrounding elastic matrix. Thus, the plastic residual component can be considered as a concentrated force acting at the center of the crack.

For the well-developed elliptical radial/median crack, Borodachev's solution [18] is adopted to formulate the residual stress intensity factor $K_{r}$, which is pertinent to the plastic residual component. To estimate the stress intensity factor $K_{e}$, pertinent to the elastic stress field, a weight function method proposed by Lambert and Glinka [19] is used. A total stress intensity factor for the system is obtained based on the condition of crack equilibrium (crack stable state) by equating the total stress intensity factor to the fracture toughness $K_{c}$. 
To cover the full range of the crack propagation, three crack configurations are considered and the solutions corresponding to each of the configurations are developed respectively. The first crack configuration is a well-developed half-elliptical crack with a depth less than $7 \%$ of the coating thickness. The second configuration is a well-developed half-elliptical crack deeper than $7 \%$ of the coating thickness but still confined within the coating layer. The third configuration is the radial/median crack penetrating the coating into the substrate. In the first case, the $7 \%$ demarcation is based on a previous experimental observation according to which the coating hardness is not affected by the substrate material properties [20]. For the second case, the appropriate modifications to the fracture toughness formula are made with the introduction of the composite hardness and an interface correction factor accounting for the influence of the substrate material properties and the presence of the coating/substrate interface. With regards to the third case, based on the condition of deformation compatibility across the coating/substrate interface, the bulk Young's modulus of the coating/substrate system is developed and included as part of the analysis.

Furthermore, a series of Vickers indentation tests at different load levels were performed on the specimen of a brittle coating/ductile substrate system, which is $\mathrm{WC} / 10 \mathrm{Co} / 4 \mathrm{Cr}$ coating/1080 low carbon steel substrate. These experiments were executed for multiple purposes. Firstly, they allowed establishing the material parameters of the coating/substrate system in question. Secondly, they confirmed the assumption made in the proposed fracture toughness model that radial/median cracks were induced in a shape of ellipse under the indentation load. Thirdly, they investigated whether the radial/median cracks would be confined within the coating layer or penetrate into the 
substrate through the interface. Fourthly, they provided dimension data on the cracks including crack lengths and depths. The obtained information and data were used to determine the fracture toughness of the tested coating/substrate system, in terms of the proposed fracture toughness model. Finally, based on the indentation experiments the relationship between $c^{3 / 2}$ (where $c$ is a radial crack length) and the applied indentation load $P$ was established to confirm its non-linearity, which is demonstrated with the proposed fracture toughness model. Optical microscopy and focused ion beam technique (FIB) were utilized to obtain the crack profile and crack dimension data, corresponding to a particular indentation load.

Based on experimental observations, material property data and crack dimensions of the $\mathrm{WC} / 10 \mathrm{Co} / 4 \mathrm{Cr}$ coating/1080 steel substrate system, the fracture toughness of this coating/substrate system was determined in terms of the proposed fracture toughness model. This fracture toughness value was then compared with the known fracture toughness value of a similar coating/substrate system (WC/12Co coating/1020 steel substrate) to validate the proposed fracture toughness model. Other existing fracture toughness models proposed by Lawn et al. [11] and Smith and Scattergood [21] were also applied to determine the fracture toughness for the $\mathrm{WC} / 10 \mathrm{Co} / 4 \mathrm{Cr}$ coating/1080 steel substrate system, utilizing the experimental data. These fracture toughness values were also compared to the known fracture toughness value of the similar coating/substrate system for discussion.

The main tasks towards the completion of the objectives can be summarized as follows: 
1. Define the domain for a brittle coating/ductile substrate system under a point indentation load; formulate the problem in consideration of the three crack configurations.

2. Develop a solution of the fracture toughness problem for the first crack configuration. It would correspond to a half-elliptical radial/median crack reaching its equilibrium at the coating depth less than $7 \%$ of the total coating thickness, in terms of the fracture mechanics approach to brittle monolithic materials.

3. Develop a solution of the fracture toughness problem for the second crack configuration. It would correspond to a radial/median crack propagating further into the coating but still being confined within the coating layer. The solution should include the composite hardness for the coating/substrate system and an interface correction factor in the analysis to take into account the influence of the substrate material properties and the presence of the interface on the fracture toughness.

4. Develop a solution of the fracture toughness problem for the third crack configuration. It would correspond to a radial/median crack penetrating into the substrate. The solution should incorporate the bulk Young's modulus for the coating/substrate system based on the condition of deformation compatibility across the coating/substrate interface.

5. Conduct Vickers indentation tests on a brittle coating/ductile substrate specimen (WC/10Co/4Cr coating/1080 low carbon steel substrate) at various load levels. Post-indentation treatment includes investigation of crack profiles and crack dimension data with the assistance of optical microscopy and focused ion beam (FIB) techniques. 
6. Establish the relationship between $c^{3 / 2}$ (where $c$ is a radial crack length) and the applied indentation load $P$ based on the experimental data. Furthermore, compute the parameters involved in the equation for fracture toughness evaluation in the proposed model such as crack shape ellipticity factor, crack shape influence factor and residual factor, using the crack dimension data from the tests. Then determine the fracture toughness of the tested coating/substrate system in terms of the proposed fracture toughness model.

7. Determine the fracture toughness of the tested coating/substrate system in terms of Lawn et al. model [11] and Smith-Scattergood model [21], respectively; compare these values with those from the proposed model and the known fracture toughness for a similar coating/substrate system (WC/12Co coating/1020 low carbon steel substrate); discuss these results and validate the proposed model.

\subsection{Thesis Outline}

Chapter 1 outlines the background and significance of the present research, its objectives and the utilized approaches and methodologies to accomplish the set of objectives.

Chapter 2 provides a detailed description of the currently existing models and techniques utilized in fracture toughness analysis of coating/substrate systems with a focus on stress-based indentation models for the radial/median crack regime. According to the literature review, the limitations of various models for brittle coating/ductile substrate systems used for fracture toughness assessment are summarized. Therefore, the need for improvement in the area of indentation-based fracture toughness analysis of brittle coating/ductile substrate systems is emphasized. 
Chapter 3 presents the detailed development procedures of the fracture toughness model for different crack depth scenarios in terms of fracture mechanics principles. Certain assumptions are made in developing the model for each crack depth scenario. At the end of this chapter, the fracture mechanics model for fracture toughness assessment of brittle coating/ductile substrate systems under indentation loading is established.

Chapter 4 describes the experimental procedures on a brittle coating/ductile substrate system including specimen preparation, testing facilities, operations, observations and results. It focuses on the experimental work and results which are used to verify the underlying assumptions made for developing the fracture mechanics model, as detailed in Chapter 3, and the experimental data which are subsequently used in Chapter 5 to verify the proposed model.

Chapter 5 provides the verification procedures of the proposed fracture toughness model utilizing the experimental results obtained in Chapter 4. The proposed model is compared with two other indentation-based fracture toughness models developed by previous researchers in terms of the fracture toughness value determined for the tested coating/substrate system against the known fracture toughness value of a similar coating/substrate system. A discussion on the results of the verification and comparison, together with the description of the advantages and limitations of the proposed model, is provided consequently.

Chapter 6 outlines the conclusions drawn from this research, elaborates on the contributions of this work towards improving existing solutions for coating fracture mechanics problems compared to the existing approaches. It also focuses on potential future research resulting from this work. 


\section{Chapter 2. Literature Review}

\subsection{Concepts of Fracture Toughness}

The concept of fracture toughness stems from linear elastic fracture mechanics (LEFM), where stress intensity factor $K$ is the characteristic parameter of the stress field ahead of the crack tip. For the mode I loading, i.e., under a normal tensile stress perpendicular to the crack, as shown in Figure 2-1, the stress intensity factor $K_{I}$ relates to the sample geometry, the size and location of the crack, and the magnitude of loads in the materials as follows [22]:

$\sigma_{i j}^{(I)}=\frac{K_{I}}{\sqrt{2 \pi R}} \cdot f_{i j}^{(I)}(\theta)$

where $R$ is the distance from the crack tip, $\theta$ is the angle around the crack tip, $f_{i j}^{(I)}(\theta)$ is the angular function and $\sigma_{i j}^{(I)}$ are stress components as shown in Figure 2-2.

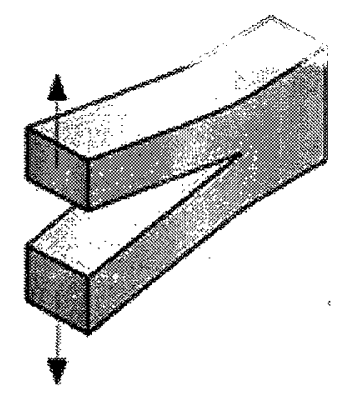

Figure 2-1. Fracture under mode I loading. 


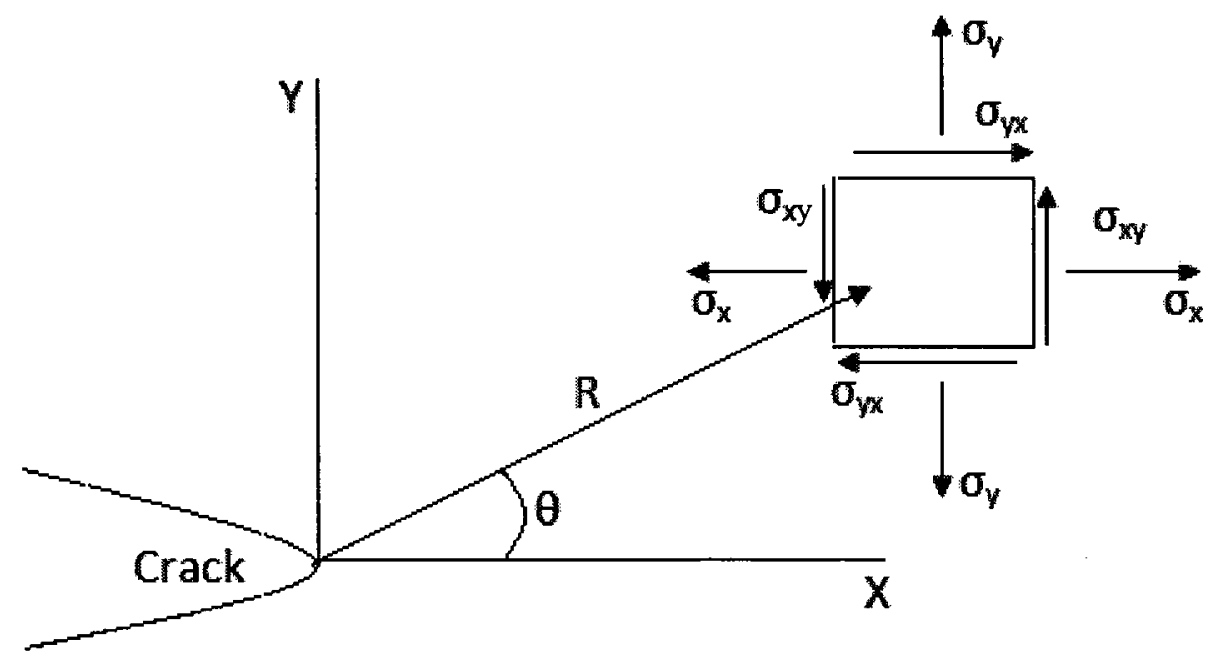

Figure 2-2. A stress field near a crack tip in a linear-elastic material.

Therefore, fracture toughness $K_{c}$, defined as the critical mode I stress intensity factor, is an indication of the amount of stress required to propagate a pre-existing flaw. It is considered to be an important material parameter for brittle materials and even ductile materials under small scale yielding conditions. It marks the ability of a material with pre-existing cracks to withstand further fracture propagation. In evaluation of fracture mechanics behavior of coated components, researchers usually utilize either stress-based or energy-based approaches. Stress-based approaches refer to determination of a critical stress intensity factor, i.e., fracture toughness, with such techniques as bending, buckling, scratching and indentation. In the energy-based approaches, a load-displacement curve, generated by an indentation, is typically utilized to establish the energy release rate.

An overview of the commonly used methodologies for fracture toughness assessment of coating systems is presented in the following sections. In particular, a 
detailed discussion on the indentation-based models for fracture toughness assessment of brittle coatings is provided.

\subsection{An Overview of Fracture Toughness Measurements for}

\section{Brittle Coatings}

\subsubsection{Indentation Technique}

\subsubsection{Indentation Fracture Toughness Evaluation for Median Cracks}

Originally, the indentation-based techniques were widely utilized to assess the fracture toughness of bulk brittle materials $[5,7]$. Lawn et al. developed a quantitative relationship to correlate the fracture toughness $K_{c}$ with the radial/median (half-penny) crack size $c_{m}$, as shown in Figure 2-3, and the indentation load $P$ applied in bulk brittle materials [11]:

$$
K_{c}=\chi_{r} \cdot\left(\frac{P}{c_{m}^{3 / 2}}\right)
$$

where $E_{b}$ and $H_{b}$ are the Young's modulus and hardness of the bulk material, respectively; $P$ is the critical load corresponding to onset of a radial fracture. $P$ and $c_{m}$ are determined from the indentation tests. The underlying assumption for this model is that the expanding spherical plastic zone of a size $b$ would act as a point force on the center of the crack. Lawn et al. used the analog of a spherical cavity under internal pressure developed by Hill [23] to relate the relative plastic zone radius $b / l$, where $l$ is a Vickers indentation half-diagonal, to the hardness-to-modulus ratio $H_{b} / E_{b}$ and the indenter angle $\psi[11]$ (see the details in Chapter 3). 
In this model, $\chi_{r}$ is a residual factor which depends on the material parameters, i.e., $\chi_{r} \approx \chi\left(\frac{E_{b}}{H_{b}}\right)^{1 / 2}$ (where $\chi$ is a coefficient of proportionality). For such well-developed radial/median cracks induced by a Vickers indenter, $\chi=0.016 \pm 0.004[5,7]$, in terms of $\chi=K_{c}\left(H_{b} / E_{b}\right)^{1 / 2} /\left(P / c_{m}^{3 / 2}\right)$. This range of $\chi$ values was initially obtained by Anstis et al. [24]. The authors averaged the data for such materials as glass-ceramic (C9606), soda-lime glass, $\mathrm{Al}_{2} \mathrm{O}_{3}$ and $\mathrm{Si}_{3} \mathrm{~N}_{4}$ with the known material properties $H_{b}, E_{b}$ and $K_{c}$ subjected to Vickers indentation testing. A series of Vickers indentation tests were also conducted on these materials to gather post-indentation crack size values $c_{m}$ corresponding to the critical loads $P$. The indentation loads were selected in such a way that the radial/median fracture pattern for these materials was well-defined; this lead to a minimum requirement of $c_{m} \geq 2 l$, where $l$ is the half-indenter diagonal, as shown in Figure 2-3. The fracture toughness values $K_{c}$ for these materials were verified by independent experiments using the double cantilever technique [24]. This constant $\chi=0.016 \pm 0.004$ was later adopted by many researchers [25-32] in the studies of brittle coating/ductile substrate systems without further verification of the acceptability of this constant for the coating systems. 


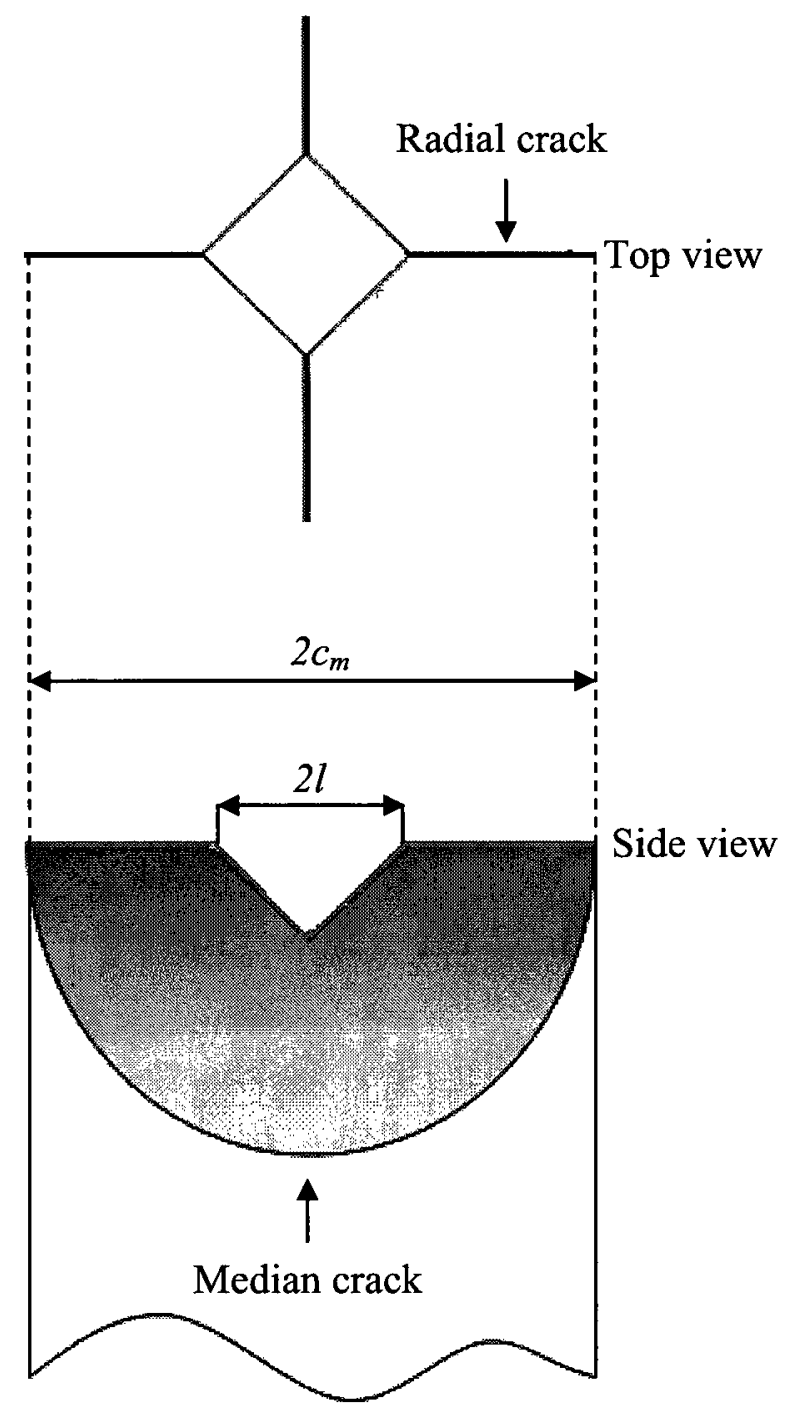

Figure 2-3. Median (half-penny) crack geometry under Vickers indentation

Li et al. [25] used Eq. (2-2) to determine the fracture toughness of high-velocity oxy-fuel (HVOF) sprayed hydroxyapatite $(\mathrm{HA})$ and $\mathrm{HA} /$ titania $\left(\mathrm{TiO}_{2}\right)$ coatings (about $80 \sim 200 \mu \mathrm{m}$ thick) on titanium alloy substrates. The authors adopted the constant $\chi=0.016$ for the model described with Eq. (2-2). Assuming that a well-developed indention crack generated by the Vickers indenter develops into a median (half-penny) shape, the authors demonstrated that the fracture toughness values increased with an increase in the amount of $\mathrm{TiO}_{2}$ in the HA coatings, from $K_{c}=0.48( \pm 0.08) \mathrm{MPa} \mathrm{m}^{1 / 2}$ to 
$0.60( \pm 0.07) \mathrm{MPa} \mathrm{m} \mathrm{m}^{1 / 2}$ and $0.67( \pm 0.06) \mathrm{MPa} \mathrm{m}^{1 / 2}$ for $0 \%, 10$ vol.\% and 20 vol.\% titania reinforcement, respectively. However, these fracture toughness values were not compared with any other known fracture toughness values for these coating/substrate systems.

Kodali et al. [26] studied the fracture toughness of amorphous diamond-like carbon (DLC) coatings on a $\mathrm{Si}(100)$ substrate. The DLC coatings were deposited in thicknesses varying from 0.7 to $7 \mu \mathrm{m}$ on the substrates using a pulse bias deposition method. Twenty microsecond pulses of $20 \mathrm{kV}$ bias were applied to the samples at about $500 \mathrm{~Hz}$ in the implantation step. The fracture response of the DLC-Si systems was studied using the Vickers indentation at loads ranging from 25 to $1000 \mathrm{~g}$. For the coating/substrate system in question, without further validation the authors assumed that the constant $\chi=0.016$ in Eq. (2-2). The radial/median cracks were selected based on the criterion: $c_{m} \geq 2 l$, where $2 l$ was the indenter diagonal. The fracture toughness values were determined to be $0.77 \pm 0.02 \mathrm{MPa} \mathrm{m}^{1 / 2}$ for a $0.7 \mu \mathrm{m}$ thick coating and $1.572 \pm 0.002 \mathrm{MPa} \mathrm{m}^{1 / 2}$ for a $7 \mu \mathrm{m}$ thick coating. The authors compared the obtained fracture toughness values to those determined by Mecholsky et al. [27] who studied the fracture toughness behavior of the polycrystalline diamond $6 \sim 12 \mu \mathrm{m}$ thick coatings grown on a silicon ( $\mathrm{Si}$ ) substrate. The comparison showed that the fracture toughness values obtained from both studies were close.

Jedrzejowski et al. [28] investigated the fracture toughness of nanocomposite (nc) TiN/SiN ${ }_{1.3}$ coatings $(1.5 \sim 2.0 \mu \mathrm{m}$ thick) deposited on crystalline silicon, (c-Si) (100), substrates using plasma enhanced chemical vapor disposition (PECVD) techniques. A Vickers indenter was used to apply an indentation load of $2 \mathrm{~N}$. Without verification for 
the coating/substrate system under investigation, the authors assumed the constant $\chi=0.016$. It was further speculated that the indenter generated a well-developed radial/median (half-penny) crack of a size $c_{m}$. The fracture toughness values, obtained using Eq. (2-2), varied from $1.8 \mathrm{MPa} \mathrm{m}^{1 / 2}$ for [Si] concentration of $5 \sim 10$ at. $\%$ to $4 \mathrm{MPa} \mathrm{m}{ }^{1 / 2}$ for $[\mathrm{Si}]>40$ at.\%. Again, these fracture toughness values were not compared with any other known fracture toughness values for these coating/substrate systems.

$\mathrm{Li}$ and Bhushan [29] characterized the fracture toughness of SiC films $(\sim 3 \mu \mathrm{m}$ thick) deposited on a $\mathrm{Si}(100)$ substrate using atmospheric pressure chemical vapor deposition (APCVD). The authors speculated that cracks developed in the SiC films in a half-penny shape and, utilizing Eq. (2-2), they determined the fracture toughness of the coating as $0.78 \mathrm{MPa} \mathrm{m}^{1 / 2}$. This fracture toughness value was found to be much lower than that of the bulk polycrystal $\mathrm{SiC}$ materials which was $3.6 \mathrm{MPa} \mathrm{m}^{1 / 2}$. In their work, the authors also adopted the constant $\chi=0.016$ based on the argument that this constant corresponds to the Vickers indenter geometry, as demonstrated by Lawn et al. [11] in their studies.

Marshall and Lawn [9] proposed a fracture mechanics model for measuring stresses in tempered surfaces of bulk glass materials. The approach was based on the indentation fracture mechanics principle where the scale of micro-cracking around an impression, generated by a diamond Vickers indenter, provided a measure of resistance to crack extension. Assuming a radial/median well-developed crack configuration in a bulk brittle material, the critical stress intensity factor $K_{c}$ for the glass system was determined (neglecting a free surface effect) as [9]: 
$K_{c}=\chi_{r} \cdot \frac{P}{c_{m}^{3 / 2}}-2 \sigma_{a p p}\left(c_{m} / \pi\right)^{1 / 2}$

where $\sigma_{a p p}$ is an applied biaxial compressive stress, $c_{m}$ is a radial/median (half-penny) crack size, $P$ is the applied indentation load corresponding to the onset of fracture and $\chi_{r}$ is the same constant as used in Eq. (2-2). In this model, for the Vickers indenter, the constant was assumed as $\chi_{r} \approx \chi\left(\frac{E_{b}}{H_{b}}\right)^{1 / 2}$ with $\chi=0.016$ according to the work reported by Lawn et al. [11]. The first term of Eq. (2-3) represents the indentation driving force on the crack, while the second term is associated with the residual resistance force. The parameters $P, c_{m}, H_{b}$ (bulk material hardness) and $E_{b}$ (bulk material Young's modulus) are determined experimentally using Berkovich or Vickers indentation tests. Plotting $\chi_{r} \cdot \frac{P}{c_{m}^{3 / 2}}$ against $2\left(c_{m} / \pi\right)^{1 / 2}$ yields a straight line, with $K_{c}$ as an interception with the ordinate axis and the applied stress $\left(-\sigma_{a p p}\right)$ as the slope. Although initially developed for bulk brittle glasses, this indentation method was utilized by some researchers to determine the fracture toughness of organic-inorganic hybrid coatings on glass [30-32].

Malzbender et al. [30] utilized Eq. (2-3) to analyze the fracture toughness of organic-inorganic hybrid coatings $(1 \sim 10 \mu \mathrm{m}$ thick) that were deposited using the spinning technique at 100 and $2500 \mathrm{rev} / \mathrm{min}$ on a glass substrate $(0.2 \mathrm{~cm}$ thick). Since the hardness to Young's modulus ratios of the coating and the substrate were similar, the stress field in the coating/substrate system was assumed to be equivalent to that of the substrate material. The authors speculated that the well-developed crack profile was 
half-penny; the constant $\chi=0.016$ was also assumed without further verification. In terms of Eq. (2-3), the fracture toughness of the substrate was determined as $K_{c}=0.62 \mathrm{MPa} \mathrm{m}{ }^{1 / 2}$ and then the same value was assumed for the coating material.

To account for the shape of radial/median cracks, Malzbender et al. [31] incorporated an empirical crack shape factor $Z$ into Eq. (2-3). The authors used it to analyze the fracture toughness of alumina filled $(1 \sim 10 \mu \mathrm{m}$ thick $)$ and silica filled (1 $\sim 10 \mu \mathrm{m}$ thick) coatings deposited on a B270 glass substrate with a spin deposition process:

$$
\begin{aligned}
& K_{c}=\chi_{r} \cdot \frac{P}{c^{3 / 2}}+Z \sigma_{a p p} c^{1 / 2} \\
& Z=1.12 \sqrt{\pi} \cdot \frac{a / c}{(3 \pi / 8)+(\pi / 8) \cdot(a / c)}
\end{aligned}
$$

where $a$ is a crack depth and $c$ is a crack length. Malzbender $e t$ al. evaluated a $Z$ shape crack factor based on the onset of delamination which commenced once the depth of the crack reached the coating/substrate interface. By measuring a radial crack length at that moment, the value of the $Z$ parameter was established as follows: $Z=0.34 \pm 0.04$ [31]. The constant $\chi=0.016$ was assumed in this work without further verification. The fracture toughness values of the alumina filled coatings were determined as $K_{c}=0.2 \pm 0.025 \mathrm{MPa} \mathrm{m}^{1 / 2}$ and $K_{c}=0.2 \pm 0.075 \mathrm{MPa} \mathrm{m}^{1 / 2}$ for the silica filled coatings, respectively. The authors recognized that the model described with Eq. (2-4 a) was originally developed for monolithic materials, and a suitable extension to the coating/substrate systems was not available. Thus, although used for the brittle coatings in this case, the model could strictly be applied only if the stress intensity factor was not 
influenced by the substrate, i.e., if the radial cracks were confined to the surface of the coating. For the cracks that penetrated deeper into the coating, the influence of the substrate and coating/substrate interface should have been taken into consideration. Hence, the author stressed that the fracture toughness values calculated with the available model were only approximate estimations [31].

Malzbender et al. [32] also analyzed the fracture toughness of 2 and $4 \mu \mathrm{m}$ thick $\mathrm{SiO}_{2}$-filled methyltrimethoxysilane (MTMS) coatings on soda-lime silica glass (Glaverbel, Brussels, Belgium) or boro-silicate glass (AF-45, Schott, Germany) substrates. The coatings were deposited using a spinning technique with free evaporation at speed between 100 and $1600 \mathrm{rpm}$. In this case, the authors excluded the shape effect factor $Z$ from the analysis, since it was assumed that radial cracks, which were a subject of the analysis, were not influenced by the presence of the substrate and the stresses were similar to those in the bulk material. This implied that the half-penny shape crack profile was assumed and the constant $\chi=0.016$ was adopted in their work without further verification. In this work, the average fracture toughness was determined as $K_{c}=0.09 \pm 0.01 \mathrm{MPa} \mathrm{m}{ }^{1 / 2}$. The authors also chose to estimate the fracture toughness using the energy-based method on the basis of chipping proposed by Li et al. [43], which will be discussed later in this chapter. The fracture toughness values showed to be similar to those using the stress-based indentation method by comparison.

Smith and Scattergood [21] in their work observed that the radial/median cracks in a bulk soda-lime glass were arrested by intercepting lateral fractures that affected the final shape of the radial/median cracks. To describe the fracture toughness of these 
cracks, the authors modified the term $\chi_{r} \cdot \frac{P}{c_{m}^{3 / 2}}$ in Eq. (2-3) to account for deviation of the crack shape from the ideal penny shape geometry. Furthermore, presuming ellipticity of the well-developed radial/median cracks with a minor axis confined by the depth of the lateral cracks, Smith and Scattergood [21] followed the work of Oore and Burns [33] to develop a crack shape correction factor. Oore and Burns developed a so-called "0 integral" based on the weight function method [33]. The "0 integral" is a general expression for the stress intensity factor and potentially could be used to predict the fracture toughness of an embedded crack of an arbitrary shape in an infinite solid under any normal stress distributions. To deduce this expression, solely empirical assumptions were utilized and direct verification of the integral was not performed due to the lack of available solutions for the irregular cracks. Based on the "0 integral" solution, a shape correction factor $f_{\text {res }}$ was incorporated in the model by Smith and Scattergood [21] as follows:

$$
K_{c}=1.22 f_{r e s} \cdot \chi_{r} \cdot \frac{P}{c^{3 / 2}}+\sigma_{a p p} \cdot c^{1 / 2}
$$

where

$f_{r e s}=\left[\frac{1}{\pi} \int_{0}^{\pi} \frac{\left(1-k^{2} \cos ^{2} \varphi\right)^{1 / 2}}{1-k^{2} \sin ^{2} \varphi} d \varphi\right]^{-1 / 2}$

$\sigma_{a p p}$ is an applied stress, $k^{2}=1-\left(\frac{a}{c}\right)^{2}$ and $0<\varphi \leq 2 \pi$. Angle $\varphi=0$ or $\pi$ corresponds to the radial (or surface) cracks $c$. To utilize Eq. (2-5 a), the crack ellipticity $a / c$ was established through a scaling relationship between the lateral crack depth $a$ and the 
indentation plastic zone size, hence, with $P^{1 / 2}$; in other terms, $a$ is proportional to $P^{1 / 2}[21]$. The relation for crack ellipticity was then:

$\frac{a}{c}=\frac{\lambda P^{1 / 2}}{c}$

where $\lambda$ was deemed as an adjustable parameter, provided that lateral crack depth $a$ was experimentally measured. Based on their experimental results, $\lambda$ values varied between $1.2 \times 10^{-5} \mathrm{~m} / \mathrm{N}^{1 / 2}$ and $1.8 \times 10^{-5} \mathrm{~m} / \mathrm{N}^{1 / 2}$. In view of the fact that for all $\lambda$ values which were less than $\lambda_{\max }=1.8 \times 10^{-5} \mathrm{~m} / \mathrm{N}^{1 / 2}$, the fracture toughness value for soda-lime glass was unrealistically low, Smith and Scattergood [21] adopted $\lambda_{\max }$ as the "best-fit" value. For the maximum $\lambda_{\max }$ value of $1.8 \times 10^{-5} \mathrm{~m} / \mathrm{N}^{1 / 2}$, the average value of the fracture toughness at different indentation loads was determined as $0.54 \pm 0.07 \mathrm{MPa} \mathrm{m}^{1 / 2}$. Apparently, as $\lambda$ decreases, the ellipticity increases and the effect of the crack shape correction factor becomes more pronounced. However, as shown by Smith and Scattergood [21], there was, in general, a poor prediction of fracture toughness for a low aspect ratio $(a / c)$ elliptical crack. This may be attributed to the fact that there is a strong change in the stress intensity factor as $a / c$ approaches zero [34]. Hence, the accuracy of the empirical weight function method in this region is uncertain $[34,35]$.

On the basis of dimensional analysis of the underlying fracture mechanics principles and experimental data, Evans and Charles [39] showed that the relationship between fracture toughness $K_{c}$ and a crack-to-indent size ratio $c_{m} / l$ exhibited a universal behavior for bulk brittle materials as follows:

$\left(K_{c} / H_{b} \cdot l^{1 / 2}\right) \cdot\left(H_{b} / E_{b}\right)^{2 / 5} \propto\left(c_{m} / l\right)^{-3 / 2}$ 
where $H_{b}$ and $E_{b}$ are Vickers hardness and the Young's modulus of the bulk brittle material respectively, and $l$ is a half-diagonal of the Vickers indentation. By plotting $\left(K_{c} / H_{b} \cdot l^{1 / 2}\right) \cdot\left(H_{b} / E_{b}\right)^{2 / 5}$ against $c_{m} / l$ and fitting the plot to the literature data provided by Evans and Charles [39] and Evans and Wilshaw [36], Niihara et al. [37] derived the best fitting constant to these data for a median crack regime $\left(c_{m} / l \geq 2.5\right)$ as 0.0711 .

To optimize the cavitation erosion resistance, Lima et al. [38] tested thermally sprayed $\mathrm{WC} / 12 \% \mathrm{Co}$; as-sprayed (AS) $50 \%(\mathrm{WC} / 12 \% \mathrm{Co})+50 \%(\mathrm{NiCr})$ and post-melted (PM) $50 \%(\mathrm{WC} / 12 \% \mathrm{Co})+50 \%(\mathrm{NiCr})$ coatings on an AISI 1020 steel substrate according to the ASTM G 32-85 standard. The best cavitation erosion resistance was exhibited by $50 \%(\mathrm{WC} / 12 \% \mathrm{Co})+50 \%(\mathrm{NiCr})$ which also exhibited the highest fracture toughness. To estimate the fracture toughness of these coating/substrate systems, Lima et al. [38] assumed that well-developed indentation cracks formed in a half-penny shape. The authors [38] determined experimentally the indentation load $P$ to fracture and the radial crack length $c_{m}$. The authors adopted the best fit constant of 0.0711 and calculated the fracture toughness in terms of Eq. (2-7) as follows:

$$
\begin{aligned}
& K_{c}=1.6 \pm 0.9 \mathrm{MPa} \mathrm{m}^{1 / 2} \text { for } \mathrm{WC} / 12 \% \mathrm{Co} \\
& K_{c}=21 \pm 14 \mathrm{MPa} \mathrm{m}^{1 / 2} \text { for as-sprayed } 50 \%(\mathrm{WC} / 12 \% \mathrm{Co})+50 \%(\mathrm{NiCr}) ; \text { and } \\
& K_{c}=32 \pm 12 \mathrm{MPa} \mathrm{m}^{1 / 2} \text { for post-melted } 50 \%(\mathrm{WC} / 12 \% \mathrm{Co})+50 \%(\mathrm{NiCr})
\end{aligned}
$$

Again, Eq. (2-7) was proposed for bulk brittle materials, and the authors did not verify the underlying assumptions regarding the well-developed crack geometry for the coating systems under investigation. 


\subsubsection{Indentation Fracture Toughness Evaluation for Palmqvist Cracks}

Recognizing the complexity of indentation fracture patterns and the observed deviation from the predictions of the models proposed by Lawn et al. [11] and Evans and Charles [39] for median cracks $c_{m}$ in the regime of $c_{m} / l \leq 3$, where $l$ is the indenter half-diagonal, Niihara et al. [40] postulated that cracks forming during indentation might have a Palmqvist configuration as shown in Figure 2-4.

Asserting such assumption about a final crack configuration allowed making more accurate predictions of the fracture toughness behavior for some bulk brittle materials than those initially established with the models proposed by Lawn et al. [11] and Evans and Wilshaw [36]. For instance, when $\mathrm{Al}_{2} \mathrm{O}_{3}$ (Lucalox), $\alpha$-SiC (sintered), $\mathrm{NaCl}$ (single crystal) and soda-lime glass were tested at Vickers indentation loads ranging between 10 and $1000 \mathrm{~g}$ [41], their fracture toughness values were predicted with $\pm 35 \%$ accuracy compared to other fracture toughness evaluation methods. 


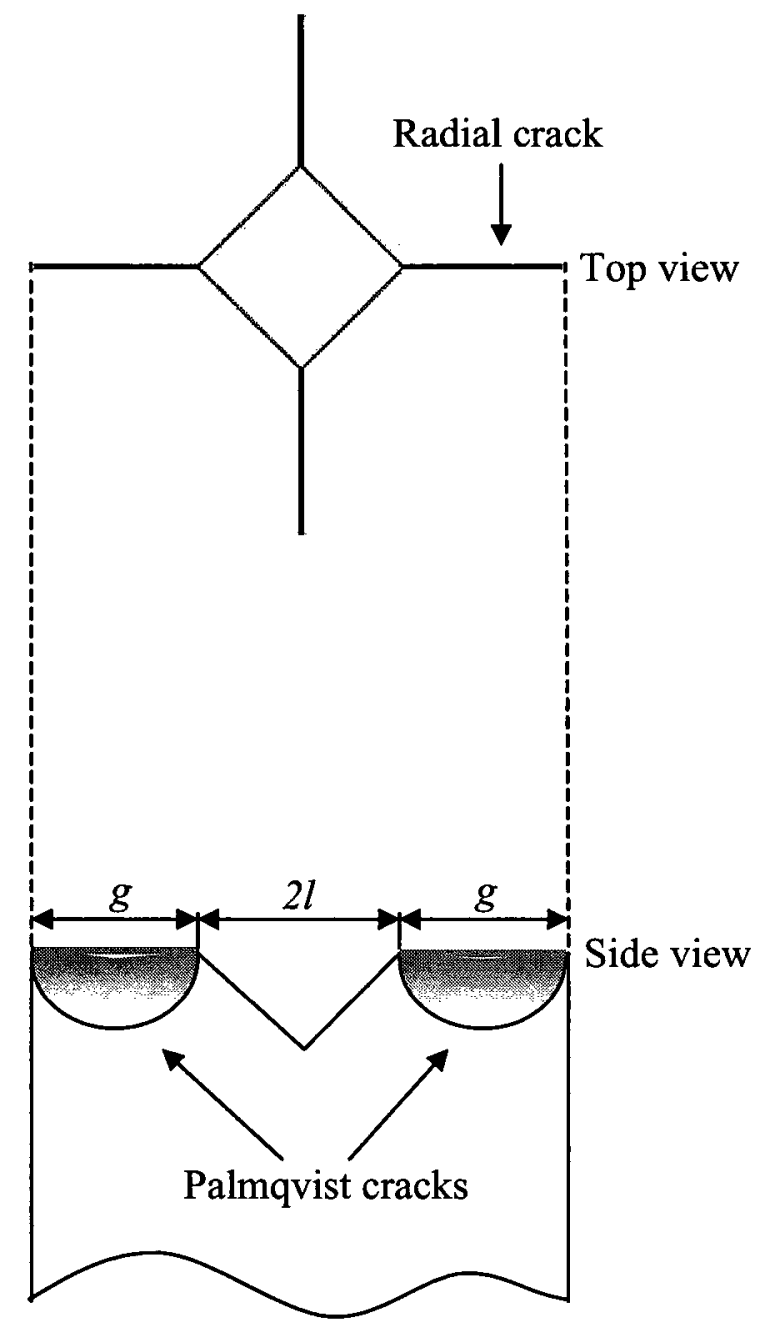

Figure 2-4. Palmqvist crack geometry under Vickers indentation.

By ascertaining that Palmqvist cracks developed in a median (half-penny) form, Niihara proposed to relate the fracture toughness $K_{c}$ of the bulk brittle material to the ratio of the Palmqvist crack length $g$, to the half-diagonal length of the indentation $l$, as shown in Figure 2-4, in the following way [37]:

$\left(\frac{K_{c} \phi}{H_{b} l^{1 / 2}}\right) \cdot\left(\frac{H_{b}}{E_{b} \phi}\right)^{2 / 5}=0.048\left(\frac{g}{l}\right)^{-1 / 2}$ 
where $E_{b}$ and $H_{b}$ are Young's modulus and Vickers hardness of the bulk brittle material respectively; and $\phi$ is a constraint factor $(\approx 3)$, and the dimensional parameters $g$ and $l$ are defined in Figure 2-4.

Campos et al. [6] used the model described with Eq. (2-8) to investigate the fracture toughness of borided AISI 1045 steels. To develop a continuous $\mathrm{Fe}_{2} \mathrm{~B}$ boride layer on the surface of AISI 1045 steel, the paste boriding process was implemented. For a layer with thickness of $46 \pm 4 \mu \mathrm{m}$, the fracture toughness was determined as $3 \pm 1 \mathrm{MPa} \mathrm{m}{ }^{1 / 2}$; for a layer with thickness of $141 \pm 6 \mu \mathrm{m}$, the fracture toughness was $2.5 \pm 2 \mathrm{MPa} \mathrm{m}{ }^{1 / 2}$.

Shetty et al. considered penny-shape Palmqvist cracks forming in some bulk brittle materials and suggested that Palmqvist cracks in equilibrium with the post-indentation crack opening due to the residual plastic zone are equivalent to a through crack in equilibrium with a wedge acting as an opening force [8]. The fracture toughness $K_{c}$ for bulk brittle materials was related to the ratio of the Palmqvist crack length $g$ to the half-diagonal length of the indentation $l$ in the following way [8]:

$$
K_{c}=0.0319 \cdot \frac{P}{g^{1 / 2} l}
$$

Roman et al. [42] analyzed the fracture toughness of electroless nickel phosphorus coatings (Ni-P), with an average thickness of $80 \mu \mathrm{m}$, deposited using the Kanigen industrial process on an AISI 4135 steel substrate, in terms of Eq. (2-9). The coatings were subjected to the post-deposition treatments in air at $300{ }^{\circ} \mathrm{C}$ and $600{ }^{\circ} \mathrm{C}$ for 6 hours respectively. The fracture toughness values were calculated as 
$K_{c}=1.5 \mathrm{MPa} \mathrm{m}{ }^{1 / 2}$ for the coatings post-treated at $300^{\circ} \mathrm{C}$ and $K_{c}=2.1 \mathrm{MPa} \mathrm{m}{ }^{1 / 2}$ for the coatings post-treated at $600^{\circ} \mathrm{C}$.

\subsubsection{Indentation Fracture Toughness Evaluation for Ring Cracks}

In some cases, particularly in multi-layer thin film systems, indentation cracks may form in a ring-like pattern. For such a configuration, indentation energy-based method was developed by researchers to estimate the fracture toughness $K_{c}$ of thin brittle coatings [43]. Assuming that a ring-like through-thickness crack developed in thin films under indentation (a cube or conical indenter was used), Li et al. [43] derived the relationship between the fracture toughness $K_{c}$ and the released elastic strain energy $U$, during fracture as follows:

$K_{c}=\left[\left(\frac{E_{c}}{\left(1-v_{c}^{2}\right) \cdot 2 \pi C_{R}}\right) \cdot\left(\frac{U}{h_{c}}\right)\right]^{1 / 2}$

where $2 \pi C_{R}$ is the total crack length, $E_{c}$ and $v_{c}$ are the Young's modulus and Poisson's ratio of the film respectively. The dissipated energy $U$ is regarded as the difference between an extrapolated loading-displacement curve $O A B$ (corresponding to the case when cracking does not occur) and a measured loading-displacement curve $O A C D$ [7], see Figure 2-5. A step (line $A C$ ) on the load-displacement curve is associated with the development of a new surface, i.e., a through-thickness fracture, as shown in Figure 2-5. An area under curve $A B C$ represents the energy difference before (curve $O B F$ ) and after (curve $O A C F$ ) the ring-like cracking, which is released as strain energy during the ring-like through-thickness crack formation. $D E$ is the unloading curve. 


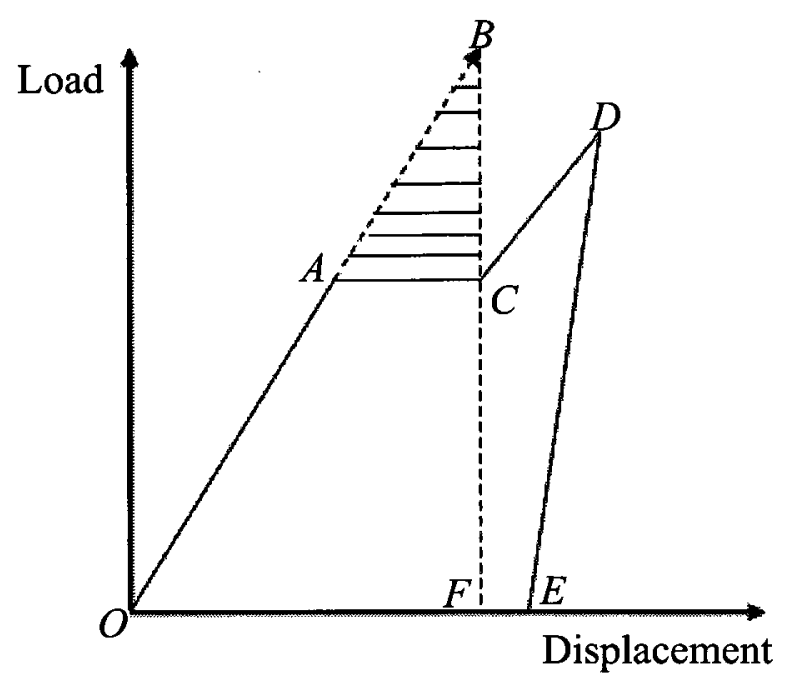

Figure 2-5. Schematic of a load-displacement curve showing a step with energy release during the loading cycle.

Neralla et al. [44] studied the fracture toughness of Ni nanoparticle-dispersed $\mathrm{Al}_{2} \mathrm{O}_{3}$ ceramic composite coatings on a silicon (100) substrate deposited by an alternating-target pulsed laser. $\mathrm{Ni}$ deposition time varied as $35,50,65$, and $80 \mathrm{~s}$ to obtain different sizes of $\mathrm{Ni}$ particles per $\mathrm{Ni}$ layer, while deposition time for $\mathrm{Al}_{2} \mathrm{O}_{3}$ was kept constant. Six layers of alumina alternating with five layers of $\mathrm{Ni}$ were deposited on a silicon (100) substrate. The average total thickness of the deposited films was around $150 \mathrm{~nm}$. Utilizing Berkovich indenter, the indentation load varied between 100 to $500 \mathrm{mN}$. Under the indentation, both ring-like through-thickness and radial cracks were observed. In the case of the ring-like cracks, the fracture toughness values for $\mathrm{Ni}$ deposition times varying between 0 to $80 \mathrm{~s}$, ranged between $1.7 \pm 0.3$ to $2.4 \pm 0.2 \mathrm{MPa} \mathrm{m} \mathrm{m}^{1 / 2}$, which were evaluated using Eq. (2-10). These fracture toughness values were also compared to those obtained with conventional means, i.e., utilizing the approach of Lawn et al. for radial/median cracks [11] described in detail in 
Section 2.2.1.1. The authors used the sum of radial cracks emanating from the three corners of an indentation mark generated by Berkovich indenter to calculate the fracture toughness. A constant $\chi=0.016$ was selected in this work without verification. The fracture toughness results yielded between $2.3 \pm 0.5$ to $3.2 \pm 0.7 \mathrm{MPa} \mathrm{m}{ }^{1 / 2}$, which were similar to the predictions obtained with Eq. (2-10).

Using Eq. (2-10), $\mathrm{Li}$ and Bhushan [45] evaluated the fracture toughness of $100 \mathrm{~nm}$ thick amorphous carbon coating on a Si substrate deposited by filtered cathodic arc (FCA), direct ion beam (IB), electron-cyclotron resonance plasma chemical vapour (ECR-CVD) and sputter (SP) deposition processes, respectively. Using a cube indenter, the specimens were loaded with a force of about $75 \mathrm{nN}$. At all loads, the indenter penetrated the coating into the substrate generating through-thickness cracks. For the FCA coating, the fracture toughness was $K_{c}=11.8 \mathrm{MPa} \mathrm{m} \mathrm{m}^{1 / 2}$; for the IB coating $K_{c}=4.3 \mathrm{MPa} \mathrm{m}{ }^{1 / 2} ;$ for the ECR-CVD coating $K_{c}=6.4 \mathrm{MPa} \mathrm{m}^{1 / 2}$; and for the SP coating $K_{c}=2.8 \mathrm{MPa} \mathrm{m}^{1 / 2}$.

Ding et al. [46] studied a multilayer system of TiN/Ti(C,N)/TiC coatings under a Vickers diamond indentation load of $10 \mu \mathrm{N}$. The following four multilayer coatings were all deposited using a CVD deposition method on cemented carbide (WC) disks with each layer of approximately $1 \mu \mathrm{m}$ thick:

(1) $\mathrm{TiN} / \mathrm{Ti}(\mathrm{C}, \mathrm{N}) / \mathrm{TiC}$,

(2) $\mathrm{Al}_{2} \mathrm{O}_{3} / \mathrm{TiC} / \mathrm{Ti}(\mathrm{C}, \mathrm{N}) / \mathrm{TiC}$,

(3) TiN/Ti(C,N)/TiC/Ti(C,N)/TiC, and

(4) TiN/Ti(C,N)/TiC/Ti(C,N)/TiC/Ti(C,N)/TiC. 
Assuming that the Vickers indenter generated the through-thickness cracks, the fracture toughness values of these coatings were determined using Eq. (2-10); the results were found to be $2.18,1.74,3.40$ and $3.90 \mathrm{MPa} \mathrm{m}^{1 / 2}$ respectively.

\subsubsection{Summary}

Due to their simplicity, indentation methods are extensively used for assessment of various mechanical properties, in particular, the fracture toughness of brittle coatings. In general, the formation of either median or Palmqvist cracks depends on the loading conditions and the type of brittle materials. For example, at higher indentation loads $(5 \sim 80 \mathrm{~N})$, radial/median cracks were observed in soda-lime glass [21]. Moreover, a median fracture mode was also observed to develop in $\mathrm{ZnSe}$ and $\mathrm{ZnS}$ [41], $\mathrm{B}_{4} \mathrm{C}$, and $\mathrm{Si}_{3} \mathrm{~N}_{4}[37,47]$ ceramics at indentation loads $\mathrm{P}<500 \mathrm{~N}$. Shetty et al. found that for bulk cemented carbides (WC/Co), both models (one based on the half-penny median crack geometry and the other on the Palmqvist crack geometry) provided very similar predictions in terms of fracture toughness behavior (within $35 \%$ similarity) [8].

The aforementioned fracture mechanics models were mostly proposed for bulk brittle materials. As for brittle coatings, on many occasions researchers speculated that an indentation crack induced by a sharp indenter (typically, Vickers or Berkovich) would develop into either a Palmqvist or a radial/median crack. Therefore, the indentation-based models, originally developed for bulk brittle materials, were often used to assess the fracture toughness of brittle coatings $[6,8,25-3238,42]$. On the other hand, the indentation method with Eq. (2-10) is applicable only for ring-like crack regime. 


\subsubsection{Bending Technique}

In addition to the indentation technique, the bending technique has also been developed for fracture toughness measurement of brittle coatings. The mechanical principle is similar to that for bulk brittle materials by employing a pre-cracked specimen subjected to a three-point bending load to induce crack propagation, though it usually needs special care in specimen preparation.

Jiang et al. [48] investigated experimentally plane strain fracture toughness of chemical vapor deposition (CVD) coatings ( $\sim 250 \mu \mathrm{m}$ thick) utilizing the three-point bending method. A diamond film was laser-cut to create a notch at one edge of the specimen, and then glued onto a brass beam. Following that, the brass beam was bent to generate a pre-crack in the film at the end of the notch. The set-up of the test for generating a pre-crack is shown in Figure 2-6.

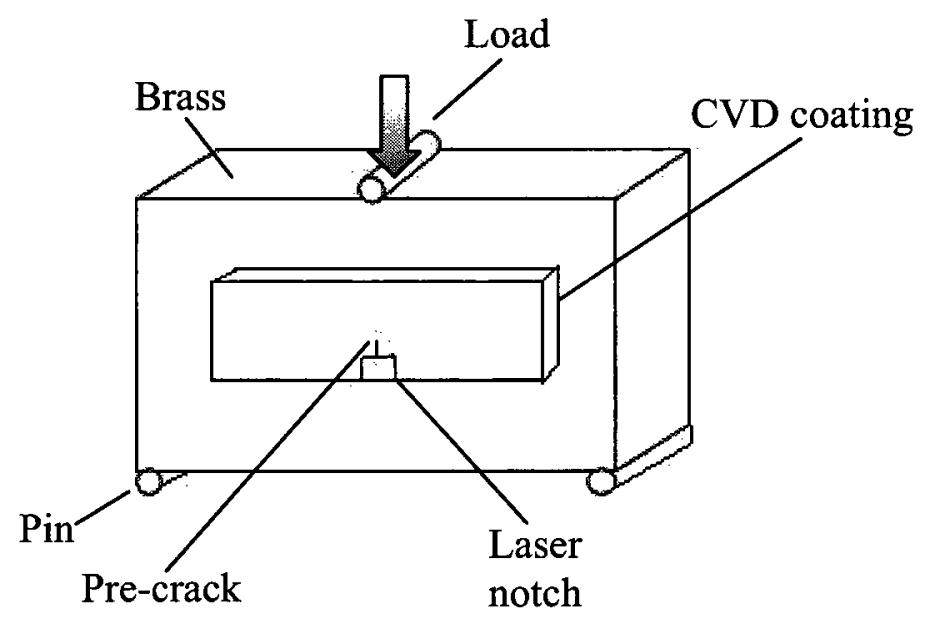

Figure 2-6. Schematic diagram of the pre-crack introduced with a bending method.

The pre-cracked specimen (i.e., the coating) was then removed from the beam and attached to a frame for the three-point bending test. The critical stress required to inflict a 
fracture was measured and then related to the fracture toughness using a well-established relationship from ASTM standard E-399 [5]:

$$
K_{c}=\left(\frac{P_{c} S}{h_{c} W^{2 / 3}}\right) \cdot f\left(a_{p c} / W\right)
$$

where $P_{c}$ is the load at fracture, $h_{c}$ and $W$ are the thickness and the width of the film respectively, $S$ is the span between the two supporting positions, $a_{p c}$ is the length of the pre-existing crack, and $f$ is a function of $a / W$, as indicated in Figure 2-7. An average value of the fracture toughness for diamond films on pre-cracked specimens, assessed with Eq. (2-11), was $K_{c}=8.0 \mathrm{MPa} \mathrm{m}^{1 / 2}$.

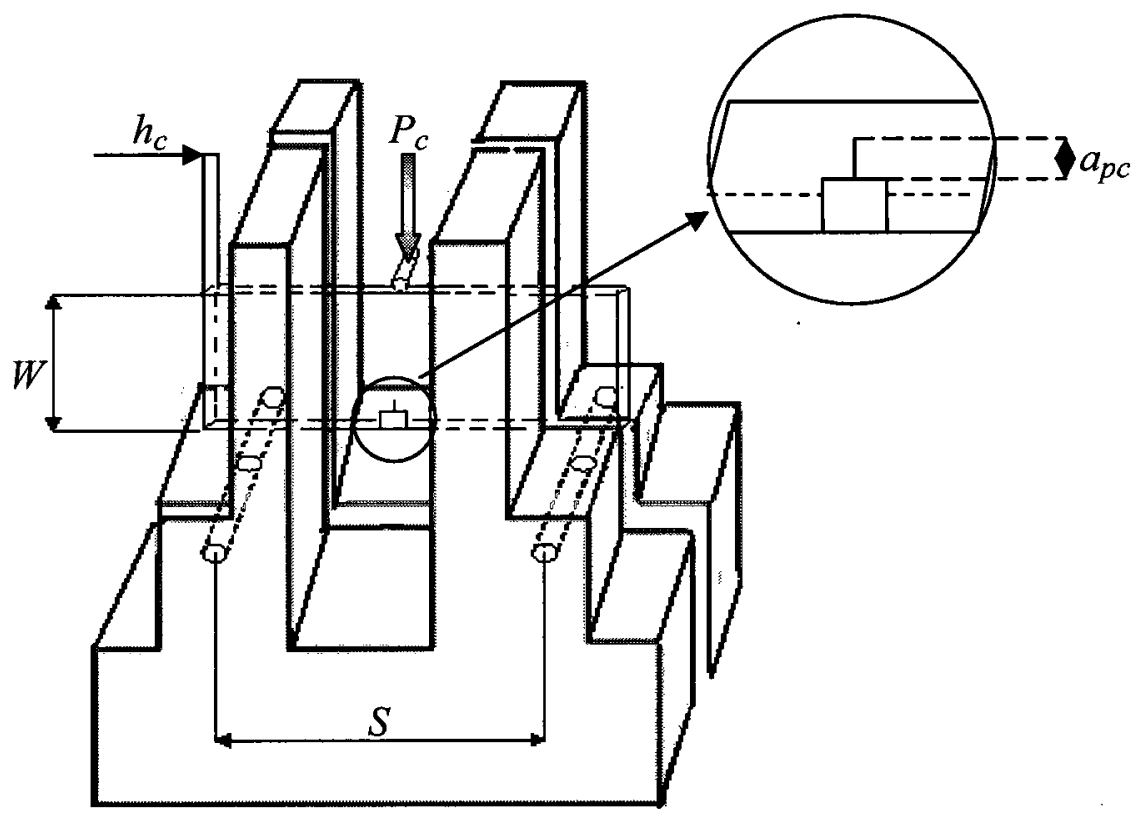

Figure 2-7. Schematic of the three-point bending test for the freestanding film with a pre-crack. 
The limitation of this method is that it is a complex procedure requiring tedious preparation. Gluing to the beam and subsequent ungluing of the pre-cracked specimen are difficult and may easily introduce errors into the fracture toughness value. Obviously, this method is not applicable for thin coatings [5].

\subsubsection{Buckling Technique}

Buckling test methods for delamination fracture toughness measurement of coated systems employ a coated specimen which is subjected to a compressive loading to induce compressive strains. The film delaminates and buckles from the substrate under the loading. The critical strain energy release rate is then related to the critical strain generated by compressive loading which is determined just before the sudden change in electrical conductivity between the film and the substrate.

Cotterell and Chen [49] employed the buckling technique to analyze the fracture behavior of indium oxide (ITO) films $(80 \sim 140 \mathrm{~nm}$ thick) under compression. Polyethylene terephthalate (PET) $0.2 \mathrm{~mm}$ thick substrates with commercially available ITO coatings were tested. The test was set-up in such a way that the compressed coated specimen could be analyzed as a plane strain beam loaded along its axis. Using the large deformation buckling theory of beams [50], the contraction ratio $\mathrm{X}$ and a radius of curvature $R_{b}$ can be determined with the following expressions:

$$
\begin{aligned}
& \mathrm{X}=2\left[1-\frac{M_{b}(t)}{K_{b}(t)}\right] \\
& \frac{L_{\text {beam }}}{R_{b}}=4 K_{b}(t) t
\end{aligned}
$$


where $K_{b}(t)$ and $M_{b}(t)$ are elliptical integrals, $t=\sin (\Theta / 2)$ is a sinusoidal function, $\Theta$ is a bending angle, $L_{b e a m}$ is the original length of the beam, $R_{b}$ is the radius of curvature, and $\mathrm{X}=e / L_{\text {beam }}$ is the contraction ratio. The radius of curvature under bending load $P_{b}$ can be calculated by measuring the shortening of the beam $e$, see Figure 2-8.

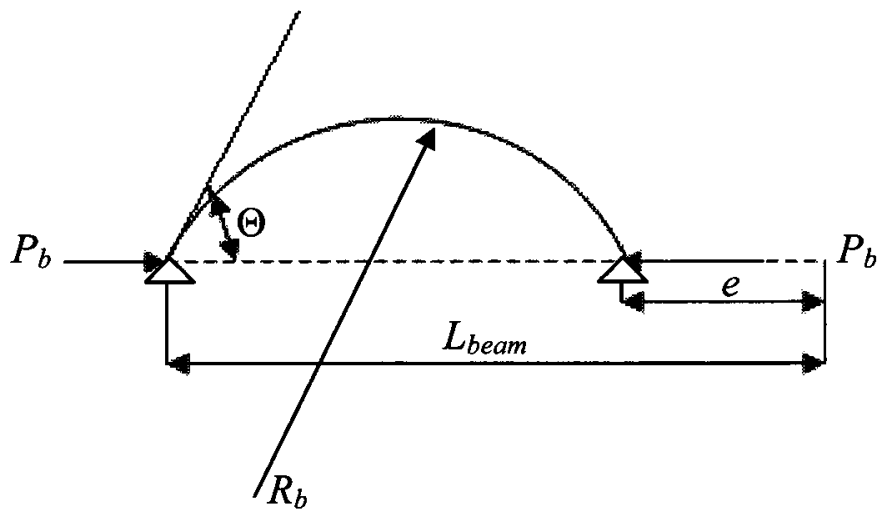

Figure 2-8. Schematic of the buckling test with simple support ends.

Due to the much smaller thickness of the film compared to that of the substrate, it could be assumed that the neutral axis of the system is near its center. Hence, the strain in the thin film could be evaluated as:

$\varepsilon_{\text {bend }}=\frac{h_{s}+h_{c}}{2 R_{b}}$

where $h_{c}$ and $h_{s}$ are the coating and the substrate thicknesses respectively. Provided that the conductivities of the film and the substrate are different, the cracking of the film can be determined from a change in electrical conductivity. The critical strain $\varepsilon_{c r i t}$, corresponding to the sudden change in resistance, is related to the critical strain energy release rate $G_{c r i t}$ as follows: 
$G_{\text {crit }}=\frac{1}{2} E_{c} \varepsilon_{c r i t}^{2} \pi h_{c} D(\alpha, \gamma)$

where $E_{c}$ is the coating Young's modulus, $D(\alpha, \gamma)$ is a function of Dundur's parameters which can be computed by finite element methods.

The PET polymer was used as a substrate in these tests due to its excellent elasticity; hence, large elastic deformation in the substrate before film fracture was possible. However, this method involves a complex numerical procedure that depends on the evaluated system.

\subsubsection{Scratching Technique}

Scratch testing is a straightforward technique for characterization of the adhesion strength of films on their substrates. During the testing process, a diamond stylus is driven across the coated surface to produce a scratch, as illustrated in Figure 2-9. Shear forces induced by an indenter distribute linearly across the coated specimen and increase with the applied load. Since mechanical properties of the coating and substrate materials are different, this would inevitably generate a discontinuity in the shear stresses across a coating/substrate interface, leading to eventual adhesive failure. Scratch tracks are observed carefully and indications of cracking within or from the scratch track are measured and recorded at the loads corresponding to the onset of fracture. Furthermore, scratch test data can be related to the fracture toughness.

Holmberg et al. [51] evaluated the fracture toughness of coatings on the basis of experimental measurements for cracking in the scratch groove in combination with finite element analysis. The authors used a three-dimensional finite element model (FEM) to simulate scratching of hard ceramic TiN coatings $(\sim 2 \mu \mathrm{m}$ thick) with a spherical rigid diamond tip. 


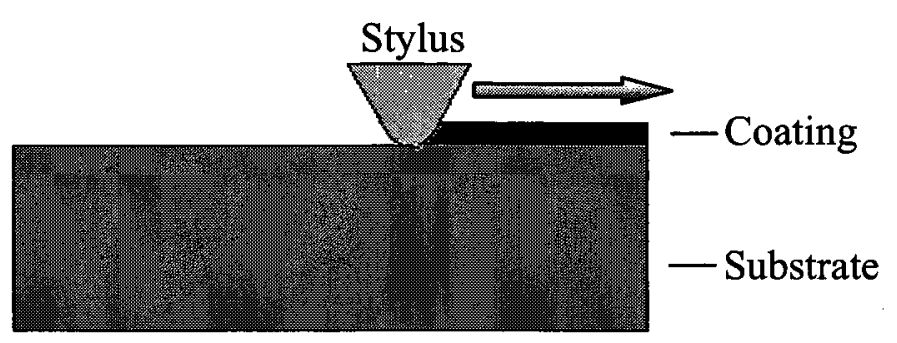

Figure 2-9. Schematic of the stylus drawn along the coated specimen surface.

The FEM model was used to determine the stresses and strains in the elastic-plastic bi-layer. The critical stress intensity factor, i.e., the fracture toughness $K_{c}$, was related to the tensile stresses $\sigma_{s c r}$ obtained through FEM modeling in the following way:

$K_{c}=\sigma_{s c r} \cdot \sqrt{b_{s p}} f_{s c r}\left(a_{s c r}, b_{s p}\right)$

where $b_{s p}$ is the crack spacing, $a_{s c r}$ is the crack length, $f_{s c r}\left(a_{s c r}, b_{s p}\right)$ is a non-dimensional function dependent on the crack length $a_{s c r}$ and the crack spacing $b_{s p}$ measured from the scratch track. Different crack types in the TiN coatings produced different fracture toughness values. The fracture toughness of transverse cracks (these are cracks that are normal to the direction of the stylus movement) in the TiN coatings was determined as $K_{c}=7 \mathrm{MPa} \mathrm{m}{ }^{1 / 2}$ with this method. This value was similar to an average fracture toughness value of $K_{c}=7.5 \mathrm{MPa} \mathrm{m}^{1 / 2}$ with variation of $\pm 1.5 \mathrm{MPa} \mathrm{m}^{1 / 2}$ for a similar TiN coating deposited on a steel substrate, which was calculated based on the experimental data provided by Wiklund et al. [52]. However, this method is not easily accessible since the function $f_{s c r}\left(a_{s c r}, b_{s p}\right)$ varies depending on the system in question. Furthermore, implementation of this technique requires rather complex FE simulation. 
Laukkanen et al. [53] conducted experimental studies with a scratch tester and three-dimensional finite element modeling (FEM) to obtain stresses and strains on a micro-level in thin titanium nitride (TiN) coatings deposited on a steel substrate under a contact load. Bi-linear hybrid elements were used in ABAQUS 5.8-14, 6.2-1 and Warp3D 14.2 finite element software packages to simulate a sphere sliding on a TiN-coated $2 \mu \mathrm{m}$ thick flat surface with an increasing normal load. The data from the scratch tests were used to calibrate the FEM results. The FEM analysis provided data about the stresses, strains and stress peak concentrations associated with potential crack locations in the coating and indicated that the cracks were subjected to general mixed-mode loading. Fracture mechanics evaluation of the crack driving force and fracture toughness were performed by deriving the stress intensity factor (SIF) solutions using boundary element analysis. The results of the finite element evaluation (particularly, peak stresses) were used as boundary conditions for the boundary element model (BEM).

The authors experimented with different types of crack fields at various densities to obtain a generic stress intensity factor (SIF) solution in a generic stress field. The SIFs were derived eventually based on the weight function approach. The weight functions for generalized loading were assumed to depend on partial derivatives of the in-plane displacements and were evaluated by the boundary element modeling (BEM) loaded with displacement boundary conditions. The SIFs were computed using a displacement correlation approach, i.e., the local near-tip displacements were used to compute the different mode SIFs. The experimentally obtained scratch densities were used as inputs for the numerical weight functions. The average fracture toughness value for the crack in 
the thin titanium nitride (TiN) coating was estimated as $4 \mathrm{MPa} \mathrm{m}^{1 / 2}[53,54]$. However, the $\mathrm{FE}$ and $\mathrm{BE}$ analyses are also very complex and require replication of the evaluation procedure for each system in question.

\subsection{Closing Remarks}

The significance of fracture toughness as a leading parameter in determining component integrity was emphasized in this chapter. An overview of existing fracture toughness measurement techniques for coating/substrate systems was reported. Special focus was placed on the existing indentation-based fracture toughness assessment models. As known from the literature review, the energy-based indentation model focuses on a system's energy state before and after fracturing of the coated system. The assumption about a ring-like through-thickness crack profile generated by an indenter limits application of this model, since from the previous studies such a crack profile does not necessarily always develop in brittle coatings.

On the other hand, indentation techniques for fracture toughness analysis of radial/median and Palmqvist cracks have also been discussed. In these indentation methods, estimation of all parameters such as hardness, Young's modulus, coating fracture load and crack length can be obtained directly from the straightforward indentation experiments. It is also evident from the literature review that to analyse the fracture toughness of a brittle coating/ductile substrate system, one often assumes that a residual factor $\chi_{r} \approx \chi\left(\frac{E_{b}}{H_{b}}\right)^{1 / 2}$ with $\chi=0.016$. However, this $\chi=0.016$ value was initially determined based on a limited pool of bulk brittle materials, as reported by Lawn et al. [11] and Anstis et al. [24]. It has not been verified for brittle coatings on 
ductile substrates. A question may be raised regarding whether this constant $\chi$ is applicable for brittle coatings on ductile substrates systems, since in most cases the fracture toughness values have not been verified by other independent means. Furthermore, to use the indentation-based models for radial/median cracks, the authors did not verify the crack profile of the well-developed crack, but speculated that it was in a half-penny shape. With the presence of the coating/substrate interface and the interference thereof, the crack shape may deviate significantly from the ideal half-penny shape geometry at higher indentation loads.

As demonstrated in the literature review, some authors attempted to address the concern regarding a change of the crack shape by incorporating a crack shape effect into the analysis $[21,31]$. However, the existing shape correction factors either have limitations in predicting crack shape behavior as the ellipticity increases [21], i.e., for the low aspect ratio elliptical crack, or are purely empirical and their valid and applicable ranges for brittle coating/ductile substrate systems have not been verified [31].

Therefore, there is an obvious demand for an indentation-based fracture toughness model which is specifically developed for brittle coating/ductile substrate systems. A fracture toughness model which takes into consideration the effects of the presence of the coating/substrate interface, material properties of the substrate, and also the crack shape geometry will essentially increase the reliability of fracture toughness assessment of brittle coating/ductile substrate systems.

It is also evident from the literature review that a derivation of a quantitative model for fracture toughness analysis of brittle coatings is governed by the assumption of a well-developed crack geometry. Since in this approach there are no imposed 
restrictions on a coating thickness, a physically realistic choice is to consider a well-developed radial/median crack geometry for the fracture mechanics model. The next chapter will focus on the development of such an indentation-based fracture mechanics model. 


\section{Chapter 3. Analytical Solutions}

\subsection{Introduction to Model Development}

This chapter presents the detailed procedures of fracture toughness model development for brittle coating/ductile substrate systems under indentation loading. It is assumed that coating and substrate media are both isotropic throughout the work. The derivation of the fracture mechanics model is based on the assumption that well-developed radial/median cracks are in elliptical shape. To deal with crack penetration in relation to the coating thickness, the fracture mechanics problem is divided into three categories based on the depth of the radial/median crack penetration into the coating/substrate system. Then, models for each category are individually developed.

The first category addresses radial/median cracks with a penetration depth less than $7 \%$ of the coating thickness. In this case, the coating hardness is presumably not affected by the substrate material properties [20], thus the problem is treated as the one for the monolithic material with the coating properties. The second category addresses cracks with a depth greater than $7 \%$ of the coating thickness but still confined within the coating. The treatment of this problem requires consideration of the influence of the substrate material properties and the coating/substrate interface as well as the crack geometry. To account for that, an interface correction factor is introduced, which is a modification of the interface correction factor given by Noda et al. [58] for an elliptical crack near a bi-material interface under remote tension. An engineering formula is proposed to describe the effects of the shear moduli ratio of substrate to coating, the distance to the interface and the position of an angle along the crack front. The third 
category addresses cracks propagating into the substrate. Based on strain compatibility across the coating/substrate interface, an expression for the bulk modulus of the coating/substrate system is obtained and incorporated into the analysis.

\subsection{Problem Description}

Consider a coating/substrate system subjected to a sharp contact under an indentation load $P$, see Figure 3-1 a. The brittle coating has a thickness $h_{c}$ with Young's modulus $E_{c}$ and Poisson's ratio $v_{c}$. The substrate has a thickness $h_{s}\left(h_{s} \gg h_{c}\right)$ with the elastic properties $E_{s}$ and $v_{s}$. Assume that the Vickers indentation generates a radial/median half-elliptical crack with the depth $a$ and the length $c(c \geq a)$, see Figure 3-1 a. In this case we follow a well-established approach [11] of treating the stress field, which is generated by a sharp indenter, as elastic-plastic in nature. According to Lawn et al. theory [11], the elastic field operates outside of the plastic zone (Figure 3-1 b) and reverses completely during the unloading half-cycle. On the other hand, the plastic component remains irreversible during the unloading half cycle (Figure 3-1 c). The plastic zone under indentation is characterized by an indenter angle $2 \psi$, a contact dimension $l$ (a half-diagonal of the Vickers indenter), a depth of the indenter penetration $d$ and a spherical expansion of the plastic zone of the radius $b$, as shown in Figure 3-1 a. 


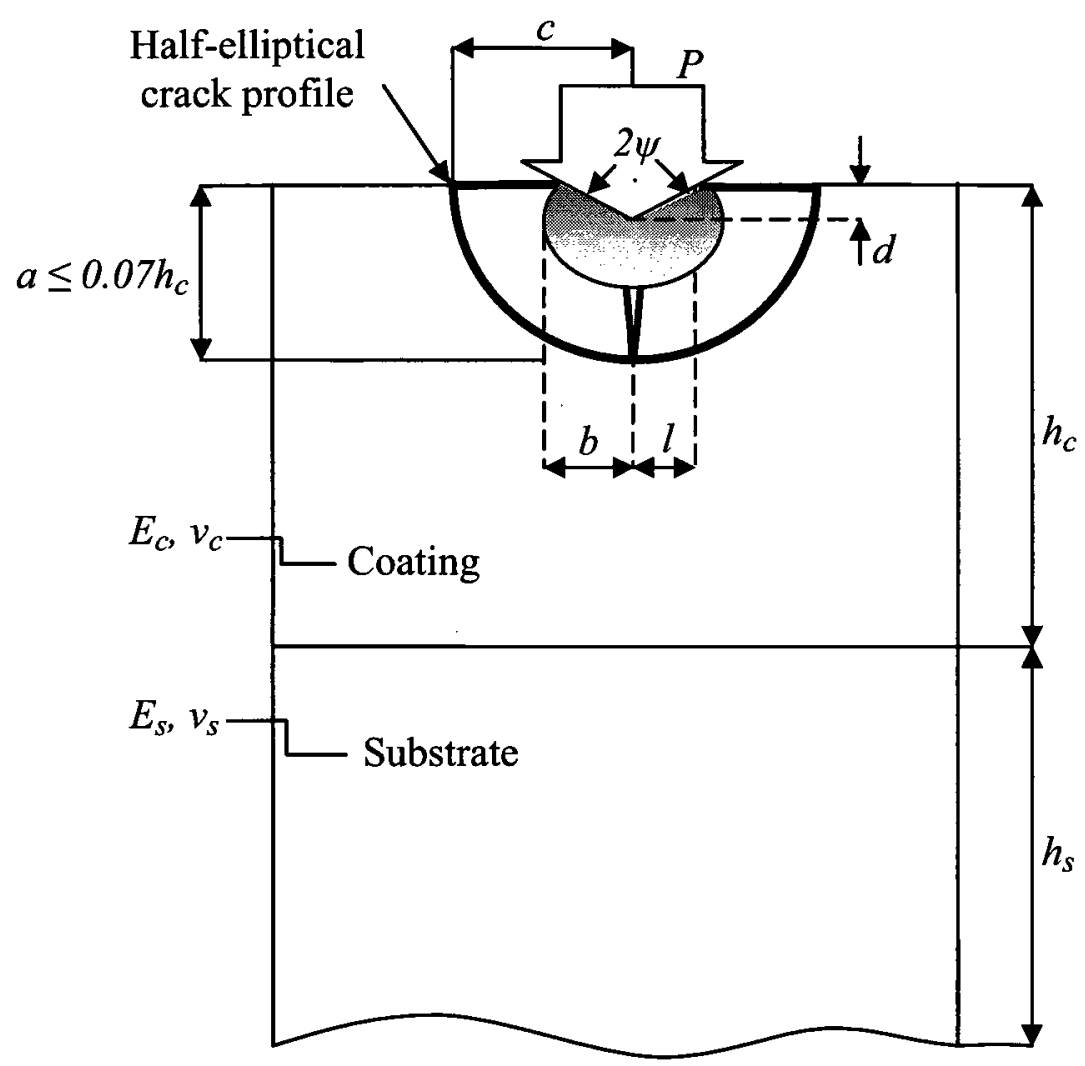

Figure 3-1 a. Schematic of a radial/median crack with the depth of $0.07 h_{c}$ or less in a brittle coating/ductile substrate system (The sketch is not scaled for clarity). 


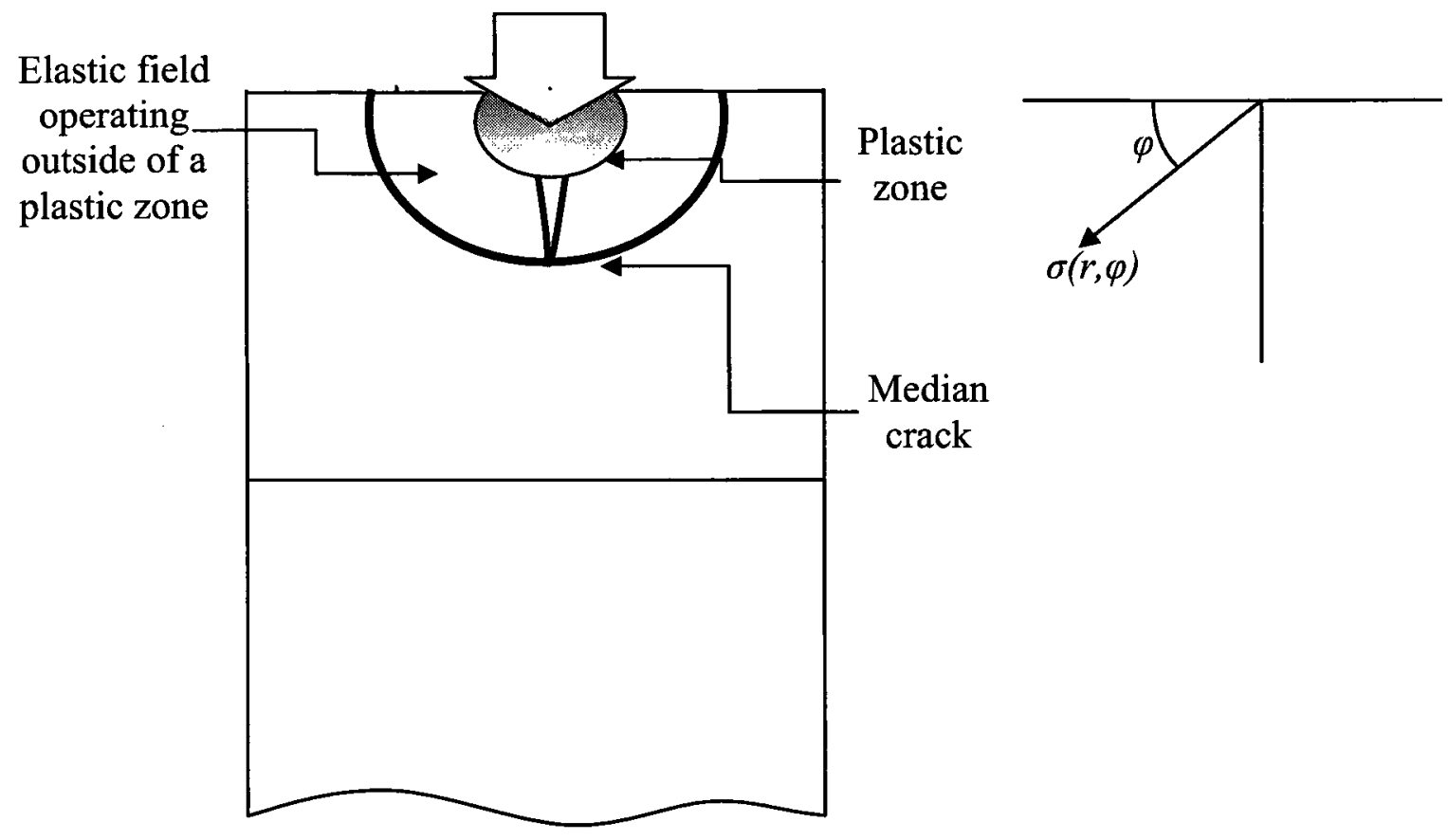

Figure 3-1 b. Schematic of an elastic component during full loading

(The sketch is not scaled for clarity). 


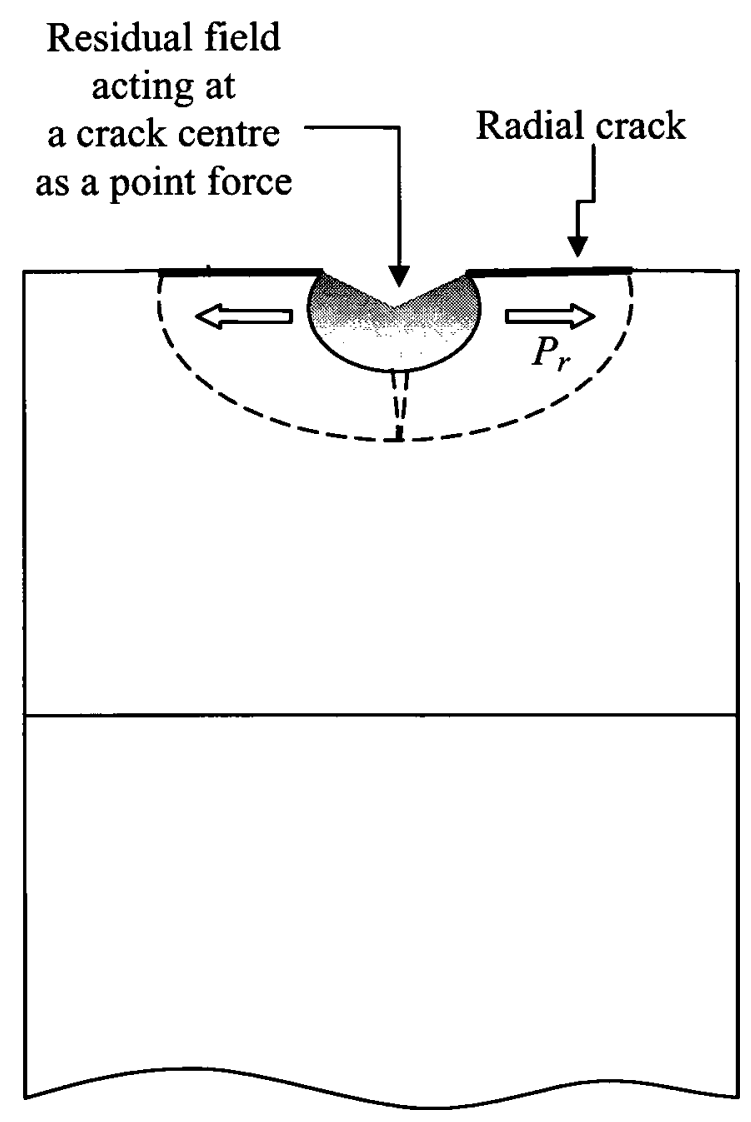

Figure 3-1 c. Schematic of a residual component after complete indenter removal (The sketch is not scaled for clarity).

\subsection{Model Development}

In consideration of crack depth variation, we divide the problem into three categories: 1) the crack depth is less than $7 \%$ of the coating thickness $h_{c}\left(a \leq 0.07 h_{c}\right)$ (Figure 3-1 a - Figure 3-1 c); 2) the crack lies in-between $0.07 h_{c}$ and a full coating thickness $h_{c}\left(0.07 h_{c}<a \leq h_{c}\right)$ and 3) the crack penetrates the substrate at a depth $d=h_{c}+d_{\text {ind }}$, where $d_{\text {ind }}$ is the depth at which the Vickers indenter penetrates into the substrate (see Figure 3-2). 


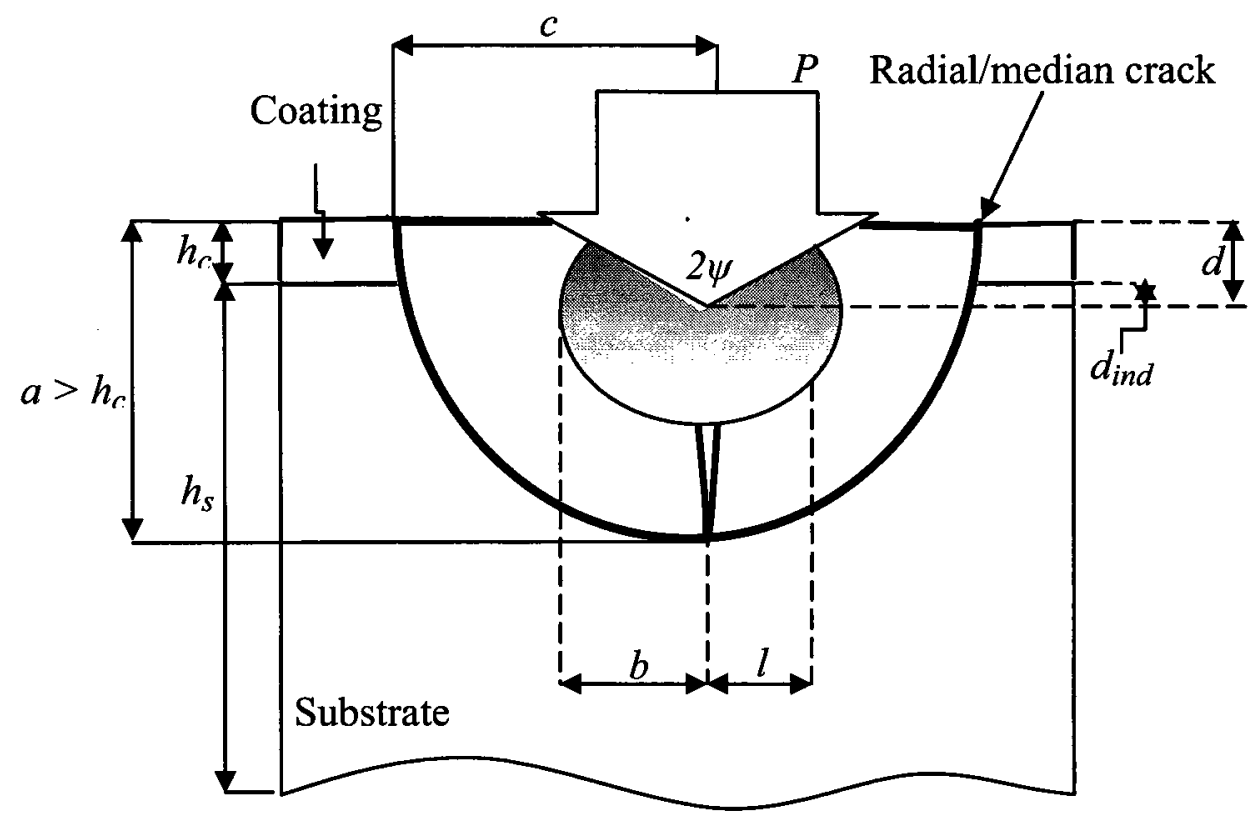

Figure 3-2. Schematic of a radial/median crack penetrating into the substrate at full loading (The sketch is not scaled for clarity).

\subsubsection{Case I: $a<0.07 h_{c}$}

Assume that a half-elliptical radial/median crack reaches its equilibrium at the coating depth less than $7 \%$ of the total coating thickness $h_{c}$, see Figure $3-1$ a. This problem can be virtually treated as the one for a brittle monolithic material with the coating properties. The expressions for the stress intensity factors pertinent to the elastic and residual components of the elastic-plastic field are considered separately.

Let us consider a residual component first. A brittle coating/ductile substrate system is deformed by an indenter over a contact length $l$ (which is equivalent to a half-diagonal of an indentation mark) and penetration depth $d$. The irreversible strains associated with the creation of the impression are accommodated by the expansion of the plastic zone at a constant volume of the material. The ratio of the volume of the 
indentation impression $\delta V$ to the volume of the plastic zone $V$ developed under the indenter can be defined as (Figure 3-1 a):

$\frac{\delta V}{V} \approx \frac{l^{2} \cdot d}{b^{3}} \approx \frac{l^{3} \cdot \cot \psi}{b^{3}}$

The plastic zone is subjected to hydrostatic compression from the surrounding elastic matrix, which is distributed across its outer boundaries. It is determined using:

$p_{b} \approx k_{c} \cdot \frac{\delta V}{V}$

where [23]

$k_{c}=\frac{1}{3} \cdot \frac{E_{c}}{\left(1-2 v_{c}\right)}$

$k_{c}$ is the bulk modulus, $E_{c}$ and $v_{c}$ are the elastic modulus and Poisson's ratio of the coating material, respectively. Thus,

$$
p_{b} \approx \frac{E_{c}}{3\left(1-2 v_{c}\right)} \cdot\left(\frac{l}{b}\right)^{3} \cdot \cot \psi
$$

It is also known that the Vickers hardness of the coating determined by indentation is described by the classic expression:

$$
H_{c} \approx \frac{P}{l^{2}}
$$

Since the plastic field remains virtually constrained by the surrounding elastic matrix, it is irreversible after complete indenter removal. Hence, the plastic zone generated underneath the indenter, can be treated as an outward force acting on the crack face. Its magnitude can be obtained by integrating the horizontal stress component over the plastic zone cross-section within the crack plane, assuming that hydrostatic pressure 
$p_{b}$ is uniformly distributed within the crack profile [11]. The combination of equations (3-4) and (3-5) yields the following expression for the residual plastic force:

$$
P_{r} \approx p_{b} \cdot b^{2}=\frac{E_{c}}{3\left(1-2 v_{c}\right)} \cdot\left(\frac{l}{b}\right)^{3} \cdot \cot \psi \cdot b^{2}=\frac{E_{c}}{3\left(1-2 v_{c}\right)} \cdot \frac{P}{H_{c}} \cdot \frac{l}{b} \cdot \cot \psi
$$

By analogy to an expanded cavity in an infinite isotropic elastic-plastic matrix [11],

$$
\frac{b}{l}=\left(\frac{E_{c}}{H_{c}}\right)^{m} \cdot(\cot \psi)^{1 / 3}
$$

where $m \approx 1 / 2[11]$.

Then, Eq. (3-6) reduces to

$$
P_{r}=\frac{P}{3\left(1-2 v_{c}\right)} \cdot\left(\frac{E_{c}}{H_{c}}\right)^{1 / 2} \cdot(\cot \psi)^{2 / 3}
$$

For well-developed cracks, i.e., $a \gg b, P_{r}$ can be treated as a force concentrated at the center point of the crack. Assuming that well-developed cracks develop into a half-elliptical shape, we further utilize the solution developed by Borodachev [18] for the stress intensity factor (SIF) of an elliptical crack subjected to a centered point load in an unbounded elastic solid. To establish the SIF, Borodachev used a semi-inverse method to solve an integral equation for mode I cracking under a point force condition. In comparison with the basic solution for a circular crack, the author introduced the crack shape influence factor $\beta$, which is a function of the crack shape ellipticity factor $k$. The $\beta$ factor was obtained using the variational formula for a solid with a crack [18] and it was tested for the well-established solution for an elliptical crack under a constant pressure. Therefore, the stress intensity factor $K_{r}$ corresponding to the residual plastic far-field force can be written (neglecting free surface effect) as [18]: 


$$
K_{r}=\frac{\beta}{\pi^{2}} \cdot \frac{P_{r}}{c^{3 / 2}} \cdot \Phi(\varphi, k) \quad(0 \leq \varphi \leq \pi)
$$

where

$$
\begin{aligned}
& \beta=\frac{\pi}{2\left(1-k^{2}\right)^{1 / 2} \cdot L(k)} \\
& L(k)=\int_{0}^{\pi / 2} \Phi(\varphi, k) \cdot\left(1+\frac{k^{2}}{1-k^{2}} \cdot \sin ^{2} \varphi\right)^{1 / 4} d \varphi \\
& \Phi(\varphi, k)=\left(1+\frac{k^{2}}{1-k^{2}} \cdot \sin ^{2} \varphi\right)^{1 / 4} \cdot\left(1-k^{2} \cdot \sin ^{2} \varphi\right)^{-1} \\
& k^{2}=1-\left(\frac{a}{c}\right)^{2}
\end{aligned}
$$

where $\Phi(\varphi, k)$ and $L(k)$ are elliptical functions.

Substituting equation (3-8) into equation (3-9 a) gives

$$
K_{r} \approx \chi_{r} \cdot \beta \cdot \frac{P}{c^{3 / 2}} \cdot \Phi(\varphi, k)
$$

where

$$
\chi_{r} \approx \frac{1}{3 \pi^{2} \cdot\left(1-2 v_{c}\right)} \cdot\left(\frac{E_{c}}{H_{c}}\right)^{1 / 2} \cdot(\cot \psi)^{2 / 3}
$$

The next step is to evaluate the influence of the elastic matrix on the crack (Figure 3-1 b), i.e., elastic component. Since the elastic field is reversible, its contribution to the crack propagation can be assessed in terms of the prior elastic contact stress distribution $\sigma(r, \varphi)$ over a prospective crack plane at $r \geq b$. Provided that a far-field solution is considered, the stresses at $r \gg>b$ can be represented by the point-load Boussinesq solution [11]: 
$\sigma(r, \varphi) \approx G_{c}(\varphi) \cdot \frac{P}{r^{2}} \quad(0 \leq \varphi \leq \pi)$

where $G_{c}(\varphi)$ is an angular function.

To obtain the stress intensity factor pertinent to the elliptical crack, subjected to radial stresses distributed between $r \geq b$ and the crack profile (i.e., the crack front), the weight function method is utilized. Lambert and Glinka [19] developed an approximate weight function $M(x, y)$ for embedded elliptical cracks in an infinite body under arbitrary loading:

$$
M(x, y)=\frac{\sqrt{2 s}}{\pi^{3 / 2} \cdot \rho^{2}} \cdot \sqrt{1-\frac{s}{8 \rho_{1}}-\frac{s}{8 \rho_{2}}-\frac{s}{8 \rho_{3}}-\frac{s}{8 \rho_{4}}}
$$

where $s$ and $\rho$ are the key parameters in the weight function expression. $s$ is the shortest distance between the load point $P^{\prime \prime}(x, y)$ and the boundary of the crack front $S^{\prime}$; and $\rho$ is the distance between the point load $P^{\prime \prime}(x, y)$ and the point $P^{\prime}\left(x^{\prime}, y^{\prime}\right)$ on the crack front $S^{\prime}$, as shown in Figure 3-3. $\rho_{1}$ through $\rho_{4}$ are parameters depending on the shape of the ellipse. They are the distances between the point $C$ and those four points on the crack front $S^{\prime}$, where the lines connecting the point $C$ to any point on the crack front are normal to the tangent of the crack front at that point. The point $C$ is the intercept between $s$ (the shortest distance from the load point $P^{\prime \prime}(x, y)$ to the crack front $S^{\prime}$ ) and the major axis of the ellipse [19] (see Figure 3-3). Using equations (3-11) and (3-12), the stress intensity factor $K_{e}$ pertinent to the elastic component can be written according to the weight function method as follows:

$$
K_{e}=\iint_{S^{\prime}} \sigma(x) \cdot M(x, y) d x d y=P \cdot G_{c}(\varphi) \cdot \iint_{S^{\prime}} \frac{M(x, y)}{r^{2}} d x d y
$$


where $S^{\prime}$ is the crack front.

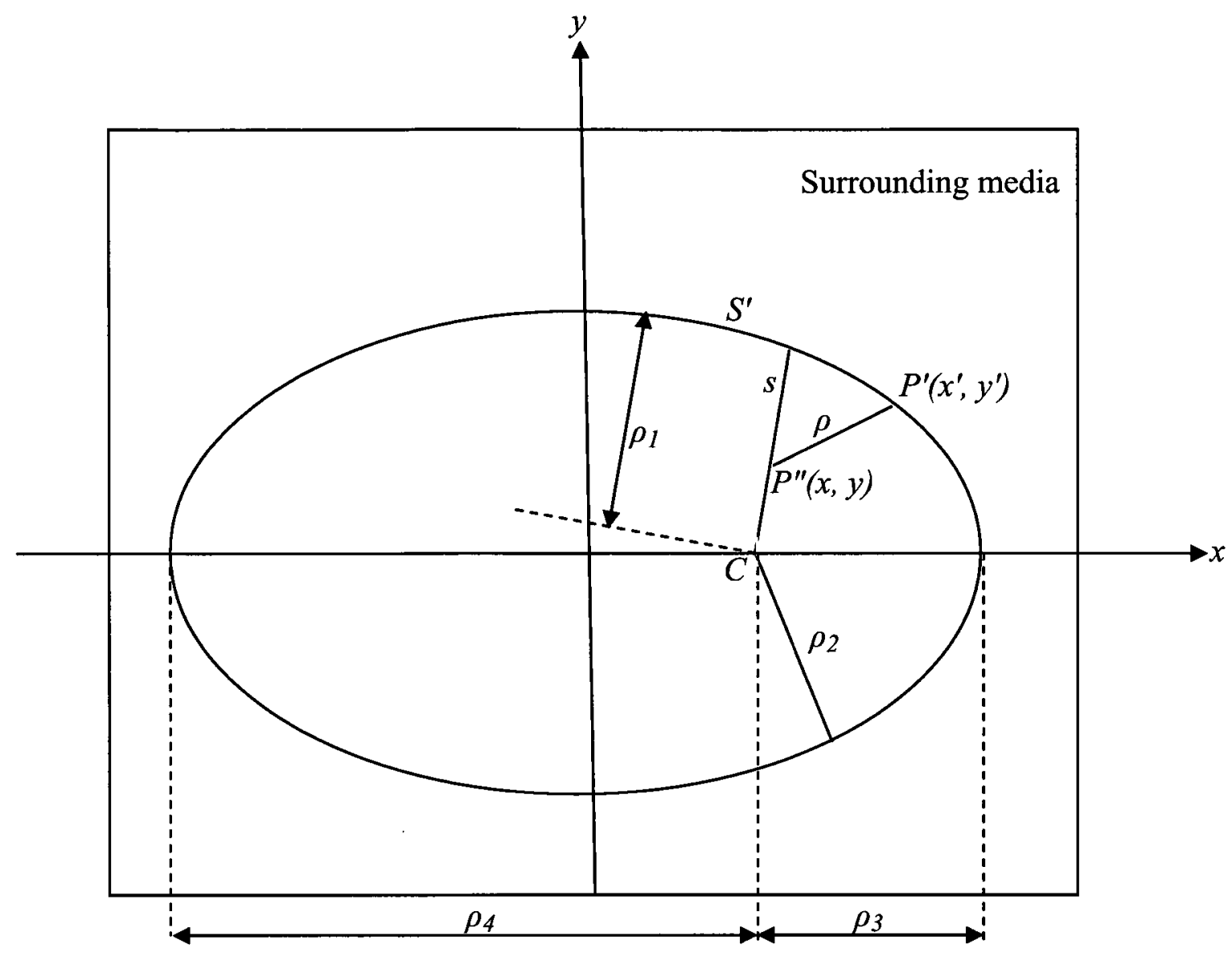

Figure 3-3. Notation for the weight function regarding an embedded elliptical crack [19].

The total stress intensity factor $K$ is defined as a superposition of both stress intensity factors $K_{r}$ and $K_{e}$. The condition of crack equilibrium is obtained by equating the total stress intensity factor $K$ to the fracture toughness $K_{c}$ as follows [11]:

$K=K_{e}+K_{r}=K_{c}$

where $K_{r}$ and $K_{e}$ are the expressions (3-10 a) and (3-13) accordingly. 
3.3.2 Case II: $0.07 h_{c} \leq a \leq h_{c}$

Consider a radial/median crack which propagates further into the coating but is still confined within it, i.e., $0.07 h_{c} \leq a \leq h_{c}$. In this case, the coating hardness may be influenced by the material properties of the substrate. Therefore, the composite hardness is introduced into the analysis. Ichimura et al. [55] described the composite hardness $H_{m}$ as a function of the film thickness $h_{c}$, the coating hardness $H_{c}$ and Young's modulus $E_{c}$, the indentation diagonal length $2 l$, the indenter half-angle $\psi$ and the substrate hardness $H_{s}$ as follows:

$H_{m}=3\left(\frac{h_{c}}{2 l}\right)\left(\frac{H_{c}}{E_{c}}\right)^{1 / 2} \cdot\left(H_{c}-H_{s}\right)(\tan \psi)^{1 / 3}+H_{s}$

Substituting equation (3-15) into equation (3-10 b) gives

$\chi_{r} \approx \frac{1}{3 \pi^{2} \cdot\left(1-2 v_{c}\right)} \cdot\left(\frac{E_{c}}{H_{m}}\right)^{1 / 2} \cdot(\cot \psi)^{2 / 3}$

while an expression for the SIF corresponding to the residual stress component persists as in Eq. (3-10 a), i.e.,

$K_{r}=\beta \cdot \Phi(\varphi, k) \cdot \chi_{r} \cdot \frac{P}{c^{3 / 2}}$

The stress intensity factor corresponding to the elastic component persists as expressed in Eqs. (3-12) and (3-13).

The crack equilibrium is defined as

$K=K_{e}+K_{r}=K_{c}$

where $K_{r}$ and $K_{e}$ are expressions (3-10 a) and (3-13) accordingly. 
To incorporate the effect of the interface on the elliptical crack located in the close proximity to the interface (such as a well-developed indentation median crack in a brittle coating/ductile substrate system), the expression (3-10 a) is further modified by an interface boundary factor $Y$. In linear elastic fracture mechanics, an engineering approach to expressing the boundary effect is as follows [56]:

$$
K=Y \sigma_{\infty} \sqrt{\frac{\pi a}{Q}}
$$

where $\sigma_{\infty} \sqrt{\pi a / Q}$ is a stress intensity factor (SIF) for an elliptical crack embedded in an infinite body under uniform stress $\sigma_{\infty}$ and $Q$ is the crack shape factor (for a through-the-thickness crack, $Q=1$; and for an elliptical crack, $Q$ will be determined in the following), and $a$ is a minor radius of an elliptical crack.

Consider an elliptical crack near a bi-material interface under uniform stress. Since the boundary factor $Y$ is independent of the load conditions, it is also applicable for the loading which is a concern of the present study, i.e., the point-force case. It is well-known that the mode I stress intensity factor of an embedded elliptical crack in an infinite medium, subjected to the remote tensile loading, can be expressed as [57]:

$$
K_{I, \infty}=\frac{\sigma_{\infty} \cdot \sqrt{\pi a}}{E(k)} \cdot\left(\sin ^{2} \xi+\left(\frac{a}{c}\right)^{2} \cos ^{2} \xi\right)^{1 / 4} \quad(0<\xi<2 \pi)
$$

where $E(k)$ is an elliptical integral of a second kind.

The stress intensity factor for an embedded elliptical crack reaches its maximum at $\xi= \pm \frac{\pi}{2}$ (Figure 3-4) and can be approximated in these points as:

$$
K_{I, \infty}=\sigma_{\infty} \sqrt{\frac{\pi a}{Q}}
$$


where

$$
Q=1+1.46 \cdot\left(\frac{a}{c}\right)^{1.65}
$$

Noda et al. have analyzed the case of an elliptical crack near a bi-material interface under remote uniform tensile stresses $\sigma_{y}^{\infty}$ and $\sigma_{z}^{\infty}$, utilizing the body-force method [58]. The configuration of the problem is shown in Figure 3-4. The authors represented a boundary factor corresponding to the mode I loading with a system of algebraic equations containing $1 / 2(n+1)(n+2)$ coefficients which could be determined by selecting a set of collocation points. The number of collocation points was $(n+1)$. The boundary correction factor $F_{I}$ for the mode I loading was related to the stress intensity factor outlined by Noda et al. as follows [58]:

$$
F_{I}=\frac{K_{I}}{\sigma_{\infty} \sqrt{\pi a}}
$$

The boundary correction factor $F_{I}$, calculated at point $A$ (see Figure 3-4), which is nearest to the interface boundary in the elliptical crack profile for a crack of the aspect ratio $\frac{a}{c}(0 \sim 1)$ and with the ratio $\frac{h}{a}(1.05 \sim \infty)$ in a bi-material system $\frac{\mu_{s}}{\mu_{c}}(0 \sim \infty)$, were calculated by Noda et al. [58]. $a$ and $c$ are minor and major radii of the ellipse; $h$ is the distance between the major axis of the elliptical crack (or the crack tips) and the coating/substrate interface; $\mu_{c}$ and $\mu_{s}$ are shear moduli of the coating and the substrate respectively, as shown in Figure 3-4. $F_{I}$ values at point $A$ are summarized in Table 3-1. 


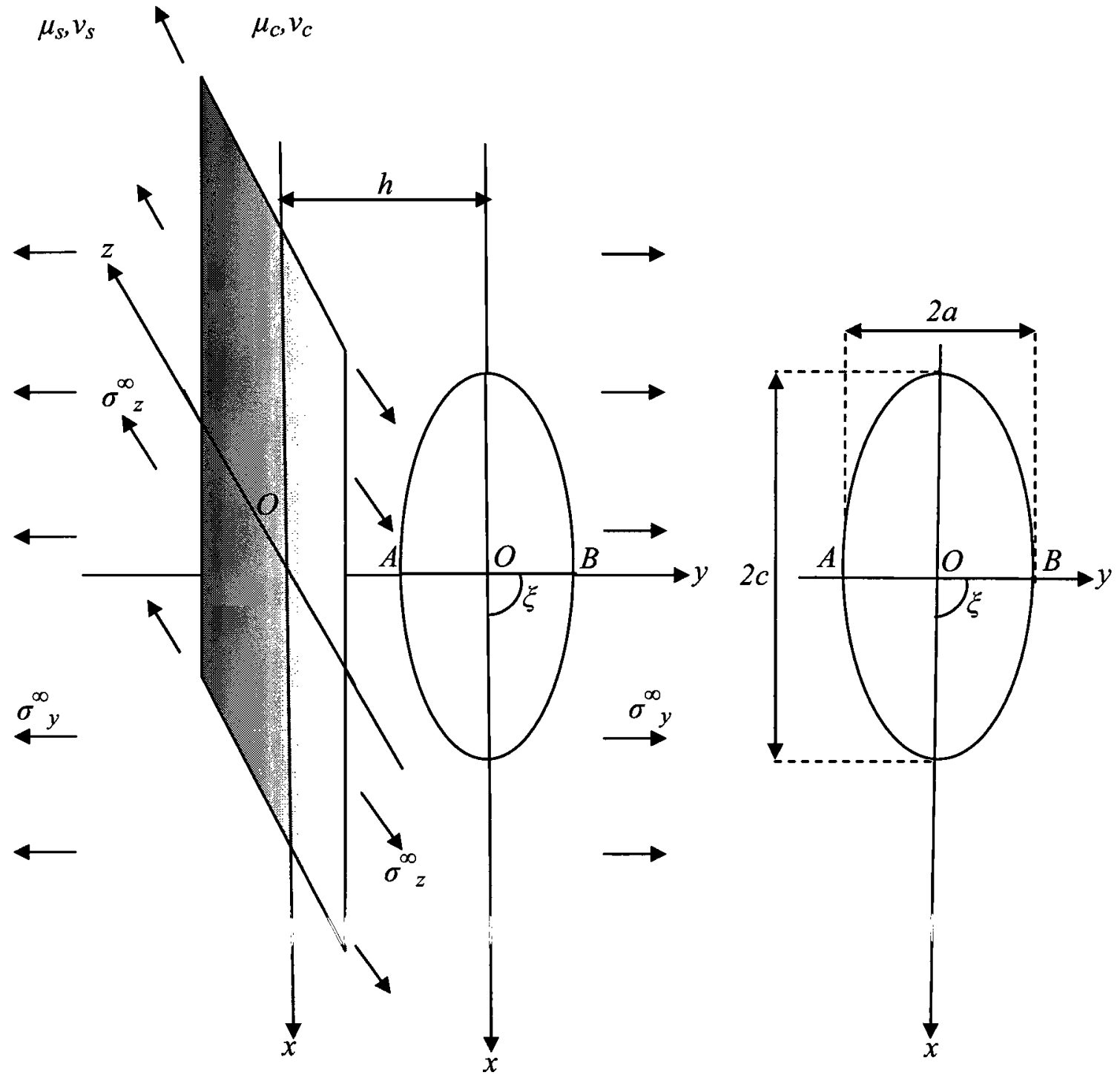

Figure 3-4. An elliptical crack perpendicular to a bi-material interface subjected to remote tensile loading [60]. 
Table 3-1. A boundary correction factor $F_{1}$ for an elliptical crack near a bi-material interface at point $A[58]$.

\begin{tabular}{|c|c|c|c|c|c|}
\hline \multirow{2}{*}{$h / a$} & \multirow{2}{*}{$a / c$} & \multicolumn{4}{|c|}{$F_{I}$} \\
\hline & & $\mu_{s} / \mu_{c}=0$ & $\mu_{s} / \mu_{c}=0.5$ & $\mu_{s} / \mu_{c}=2$ & $\mu_{s} / \mu_{c}=\infty$ \\
\hline \multirow{3}{*}{1.05} & 0 & 2.1551 & 1.2334 & 0.8212 & 0.5387 \\
\hline & 0.5 & 1.3781 & 0.9654 & 00.7070 & 0.4999 \\
\hline & 1 & 0.9066 & 0.7120 & 0.5680 & 0.4381 \\
\hline \multirow{3}{*}{1.2} & 0 & 1.4621 & 1.1136 & 0.9029 & 0.7278 \\
\hline & 0.5 & 1.0408 & 0.8866 & 0.7699 & 0.6627 \\
\hline & 1 & 0.7331 & 0.6653 & 0.6094 & 0.5550 \\
\hline \multirow{3}{*}{1.4} & 0 & 1.2535 & 1.0676 & 0.9399 & 0.8264 \\
\hline & 0.5 & 0.9337 & 0.8573 & 0.7961 & 0.7379 \\
\hline & 1 & 0.6806 & 0.6499 & 0.6239 & 0.5985 \\
\hline \multirow{3}{*}{1.6} & 0 & 1.1670 & 1.0406 & 0.9683 & 0.8774 \\
\hline & 0.5 & 0.8904 & 0.8449 & 0.8077 & 0.7719 \\
\hline & 1 & 0.6615 & 0.6441 & 0.6295 & 0.6152 \\
\hline \multirow{3}{*}{1.8} & 0 & 1.1200 & 1.0336 & 0.9691 & 0.9082 \\
\hline & 0.5 & 0.8681 & 0.8383 & 0.8138 & 0.7901 \\
\hline & 1 & 0.6522 & 0.6413 & 0.6322 & 0.6232 \\
\hline \multirow{3}{*}{2} & 0 & 1.0909 & 1.0258 & 0.9761 & 0.9285 \\
\hline & 0.5 & 0.8551 & 0.8345 & 0.8174 & 0.8010 \\
\hline & 1 & 0.6470 & 0.6397 & 0.6336 & 0.6277 \\
\hline \multirow{3}{*}{$1.00 \mathrm{E}+10$} & 0 & 1.000 & 1.00 & 1.00 & 1.00 \\
\hline & 0.5 & 0.8527 & 0.8257 & 0.8257 & 0.8527 \\
\hline & 1 & 0.6366 & 0.6366 & 0.6366 & 0.6366 \\
\hline
\end{tabular}

To consolidate the effect of the crack aspect ratio $a / c$ and to single out the effect of the interface in terms of a mode I interface correction factor $Y_{I}$, we follow an 
engineering approach described with Eq. (3-18) and further normalize the results provided by Noda et al. (see Table 3-1 and Eq. (3-22)) with the crack shape factor $\sqrt{Q}$, as follows

$$
Y_{I}=F_{I} \cdot \sqrt{Q}=\frac{K_{I} \sqrt{Q}}{\sigma_{\infty} \sqrt{\pi a}}
$$

The values of $\sqrt{Q}$ are calculated with Eq. (3-21) for different $a / c$ ratios. A summary of the $Y_{I}$ values calculated with Eq. (3-23) is provided in Table 3-2.

Table 3-2. Interface correction factor $Y_{I}=F_{I} \cdot \sqrt{Q}$ at point $A$.

\begin{tabular}{|c|c|c|c|c|c|}
\hline \multirow{2}{*}{$h / a$} & \multirow{2}{*}{$a / c$} & \multicolumn{5}{|c|}{$Y_{I}$} \\
\cline { 3 - 6 } & & $\mu_{s} / \mu_{c}=0$ & $\mu_{s} / \mu_{c}=0.5$ & $\mu_{s} / \mu_{c}=2$ & $\mu_{s} / \mu_{c}=\infty$ \\
\hline 1.05 & 0 & 2.1551 & 1.2334 & 0.8212 & 0.5387 \\
\hline 1.05 & 0.5 & 1.6681356 & 1.1685786 & 0.8557956 & 0.6051092 \\
\hline 1.05 & 1 & 1.4219465 & 1.1167284 & 0.8908732 & 0.687133 \\
\hline 1.2 & 0 & 1.4621 & 1.1136 & 0.9029 & 0.7278 \\
\hline 1.2 & 0.5 & 1.2598473 & 1.0731943 & 0.9319335 & 0.8021722 \\
\hline 1.2 & 1 & 1.1498224 & 1.0434823 & 0.9558066 & 0.8704835 \\
\hline 1.4 & 0 & 1.2535 & 1.0676 & 0.9399 & 0.8264 \\
\hline 1.4 & 0.5 & 1.130207 & 1.0377278 & 0.9636476 & 0.8931988 \\
\hline 1.4 & 1 & 1.0674794 & 1.0193283 & 0.9785489 & 0.9387106 \\
\hline 1.6 & 0 & 1.167 & 1.046 & 0.9683 & 0.8774 \\
\hline 1.6 & 0.5 & 1.077794 & 1.0227181 & 0.9776889 & 0.9343545 \\
\hline 1.6 & 1 & 1.0375222 & 1.0102314 & 0.9873322 & 0.9649035 \\
\hline 1.8 & 0 & 1.12 & 1.0336 & 0.9691 & 0.9082 \\
\hline 1.8 & 0.5 & 1.0508008 & 1.014729 & 0.9850728 & 0.9563848 \\
\hline 1.8 & 1 & 1.0229357 & 1.0058397 & 0.9776079 & 0.8276314 \\
\hline 2 & 0 & 1.0909 & 1.0258 & 0.9761 & 0.9285 \\
\hline 2 & 0.5 & 1.0350648 & 1.0101293 & 0.9894304 & 0.9695789 \\
\hline 2 & 1 & 1.0147798 & 1.0033302 & 0.9937628 & 0.984509 \\
\hline $1.00 \mathrm{E}+10$ & 0 & 1 & 1 & 1 & 1 \\
\hline $1.00 \mathrm{E}+10$ & 0.5 & 0.9994772 & 0.9994772 & 0.9994772 & 0.9994772 \\
\hline $1.00 \mathrm{E}+10$ & 1 & 0.9984681 & 0.9984681 & 0.9984681 & 0.9984681 \\
\hline
\end{tabular}


A comparison between Table 3-2 and Table 3-1 demonstrates that the effect of the crack aspect ratio $a / c$ on the interface correction factor $Y_{I}$ is minimized. Generally speaking, the influence of the interface, in terms of a mode I interface correction factor $Y_{I}$, decreases as the distance $h$ between the crack tip and the interface increases. For $\frac{\mu_{s}}{\mu_{c}}<1$, the presence of the interface magnifies the stress intensity factor, but for $\frac{\mu_{s}}{\mu_{c}}>1$, it diminishes the stress intensity factor. In order to better represent the interface effect on the stress intensity factor, engineering judgment is further utilized to derive an equation fitting the data in Table 3-2.

It is well known that for a solid with a free surface $\left(\frac{\mu_{s}}{\mu_{c}}=0\right.$, that is the substrate does not exist), the finite body effect on the stress intensity factor can be generally characterized by a factor of $\frac{1}{\cos \left(\frac{\pi a}{2 h}\right)}[59]$. This effect will decrease if the crack-containing body is perfectly bonded to another solid body. It should be equal to 1 for a system of $\frac{\mu_{s}}{\mu_{c}}=1$. To the other extreme, if the crack-containing body is interfaced with a rigid body $\left(\frac{\mu_{s}}{\mu_{c}}=\infty\right)$, the finite-body effect should be the inverse to that of a free surface, i.e., $\cos \left(\frac{\pi a}{2 h}\right)$. To cover these relations and also represent this trend with the moduli ratio, as a first approximation we propose the following formula to fit the data in Table 3-2: 
$Y_{I}=\frac{1+\frac{\mu_{s}}{\mu_{c}} \cos \left(\frac{\pi a}{2(a+h)}\right)}{\frac{\mu_{s}}{\mu_{c}}+\cos \left(\frac{\pi a}{2(a+h)}\right)}$

which is deemed to be an interface correction factor $Y_{I}$, since it only depends on the distance to the interface boundary and the nature of this boundary.

Then Eq. (3-24) is utilized to estimate values of the interface correction factor $Y_{I}$ at point $A$. This is to verify the validity of the proposed Eq. (3-24) against the values in Table 3-2. The obtained values using Eq. (3-24) at point $A$ for an elliptical crack with the aspect ratio $\frac{a}{c}(0 \sim 1)$, with the ratio $\frac{h}{a}(1.05 \sim \infty)$ in a bi-material system $\frac{\mu_{s}}{\mu_{c}}(0 \sim \infty)$, are summarized in Table 3-3. 
Table 3-3. Proposed interface correction factor $Y_{I}$ for an elliptical crack

near a bi-material interface at point $A$.

\begin{tabular}{|c|c|c|c|c|c|}
\hline \multirow{2}{*}{$h / a$} & \multirow{2}{*}{$a / c$} & \multicolumn{5}{|c|}{$Y_{I}$} \\
\cline { 3 - 6 } & & $\mu_{s} / \mu_{c}=0$ & $\mu_{s} / \mu_{c}=0.5$ & $\mu_{s} / \mu_{c}=2$ & $\mu_{s} / \mu_{c}=\infty$ \\
\hline 1.05 & 0 & 1.38788344 & 1.11449138 & 0.89727029 & 0.7205216 \\
\hline 1.05 & 0.5 & 1.38788344 & 1.11449138 & 0.89727029 & 0.7205216 \\
\hline 1.05 & 1 & 1.38788344 & 1.11449138 & 0.89727029 & 0.7205216 \\
\hline 1.2 & 0 & 1.32318962 & 1.09725284 & 0.91136698 & 0.75574958 \\
\hline 1.2 & 0.5 & 1.32318962 & 1.09725284 & 0.91136698 & 0.75574958 \\
\hline 1.2 & 1 & 1.32318962 & 1.09725284 & 0.91136698 & 0.75574958 \\
\hline 1.4 & 0 & 1.2604724 & 1.07988793 & 0.92602201 & 0.79335335 \\
\hline 1.4 & 0.5 & 1.2604724 & 1.07988793 & 0.92602201 & 0.79335335 \\
\hline 1.4 & 1 & 1.2604724 & 1.07988793 & 0.92602201 & 0.79335335 \\
\hline 1.6 & 0 & 1.21509064 & 1.06690033 & 0.93729467 & 0.82298387 \\
\hline 1.6 & 0.5 & 1.21509064 & 1.06690033 & 0.93729467 & 0.82298387 \\
\hline 1.6 & 1 & 1.21509064 & 1.06690033 & 0.93729467 & 0.82298387 \\
\hline 1.8 & 0 & 1.1810221 & 1.0569069 & 0.94615713 & 0.8467242 \\
\hline 1.8 & 0.5 & 1.1810221 & 1.0569069 & 0.94615713 & 0.8467242 \\
\hline 1.8 & 1 & 1.1810221 & 1.0569069 & 0.94615713 & 0.8467242 \\
\hline 2 & 0 & 1.15470053 & 1.0490381 & 0.95325422 & 0.86602541 \\
\hline 2 & 0.5 & 1.15470053 & 1.0490381 & 0.95325422 & 0.86602541 \\
\hline 2 & 1 & 1.15470053 & 1.0490381 & 0.95325422 & 0.86602541 \\
\hline $1.00 \mathrm{E}+10$ & 0 & 1 & 1 & 1 & 1 \\
\hline $1.00 \mathrm{E}+10$ & 0.5 & 1 & 1 & 1 & 1 \\
\hline $1.00 \mathrm{E}+10$ & 1 & 1 & 1 & 1 & 1 \\
\hline
\end{tabular}

Since the effect of the crack aspect ratio $a / c$ on the interface correction factor $Y_{I}$ is excluded in Eq. (3-24), which is consistent with Eq. (3-23), the values in Table 3-3 do not vary with the $a / c$ ratio. To examine how Eq. (3-24) (i.e., the values from Table 3-3) fits the data in Table 3-2 (i.e., the values calculated with Eq. (3-23)), the comparison of these values is shown in Figure 3-5. 


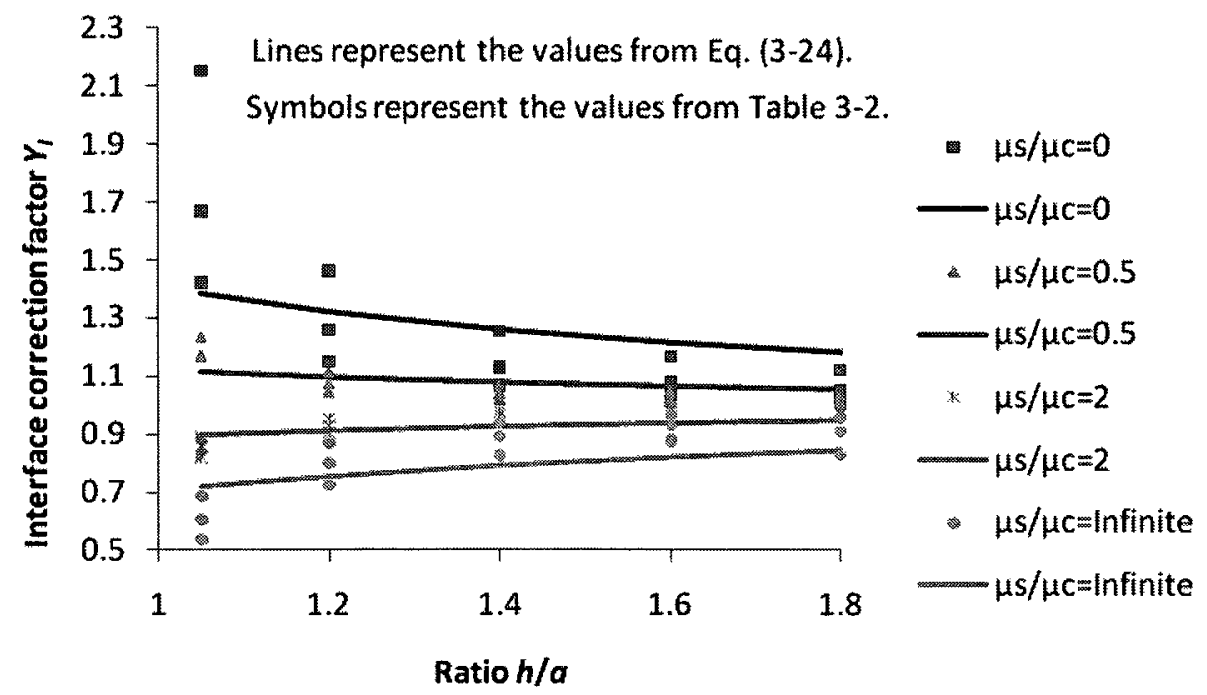

Figure 3-5. Comparison between the proposed interface correction factor $Y_{I}$ predicted with Eq. (3-24) to those values calculated with Eq. (3-23).

It is obvious that in a general case, there is a very good correlation between the proposed interface correction factor $Y_{I}$ described with Eq. (3-24) and the normalized interface correction factor $F_{I} \cdot \sqrt{Q}$ of Noda et al. defined with Eq. (3-23), except for the near interface configuration in a system with $\frac{\mu_{s}}{\mu_{c}}=0$ and $\frac{\mu_{s}}{\mu_{c}}=\infty$. Since for most coating/substrate systems, the moduli ratio $\frac{\mu_{s}}{\mu_{c}}$ usually falls between $0.5 \sim 2$, further refinement of this interface correction factor is not pursued in this work.

Furthermore, to describe the influence of the interface on the stress intensity factor along the whole "lower" part of the elliptical crack front under mode I loading, we further propose to expand the interface correction factor $Y_{I}$ (Eq. (3-24)) to include an angular effect as follows: 


$$
Y_{I a}=\frac{1+\frac{\mu_{s}}{\mu_{c}} \cos \left(\frac{\pi a \sin \xi}{2(a \sin \xi+h)}\right)}{\frac{\mu_{s}}{\mu_{c}}+\cos \left(\frac{\pi a \sin \xi}{2(a \sin \xi+h)}\right)} \quad(\pi<\xi<2 \pi)
$$

To confirm validity of the proposed angular interface correction factor $Y_{I a}$ in the above equation, the predictions made with Eq. (3-25) are compared by fitting to the results obtained by Noda et al. [58] for the following cases: $a / c=0.5, h / a=1.2$, $\mu_{s} / \mu_{c}=0.5$ and $a / c=0.5, h / a=1.2, \mu_{s} / \mu_{c}=2$. These two cases were selected for further studies, since for most coating/substrate systems the ratio between shear moduli $\mu_{s} / \mu_{c}$ falls within the range of 0.5 to 2 .

For the purposes of comparison, the $F_{I}$ values [58] for the elliptical crack configurations, that is $a / c=0.5, h / a=1.2, \mu_{s} / \mu_{c}=0.5$ and $a / c=0.5, h / a=1.2$, $\mu_{s} / \mu_{c}=2$, were gathered with the xyExtract, version 2.3 software which is used to extract data from two-dimensional graphs. In this study, the graph from the article of Noda et al. was scanned and saved in a bitmap format and then read by the xyExtract software which converted the graph back to xy-data file as shown in Figure 3-6. It is important to note that in the work of Noda et al. the angle $\pi<\beta<2 \pi$ shown in Figure 3-6 corresponds to the angle $\pi<\xi<2 \pi$ in the present work. 


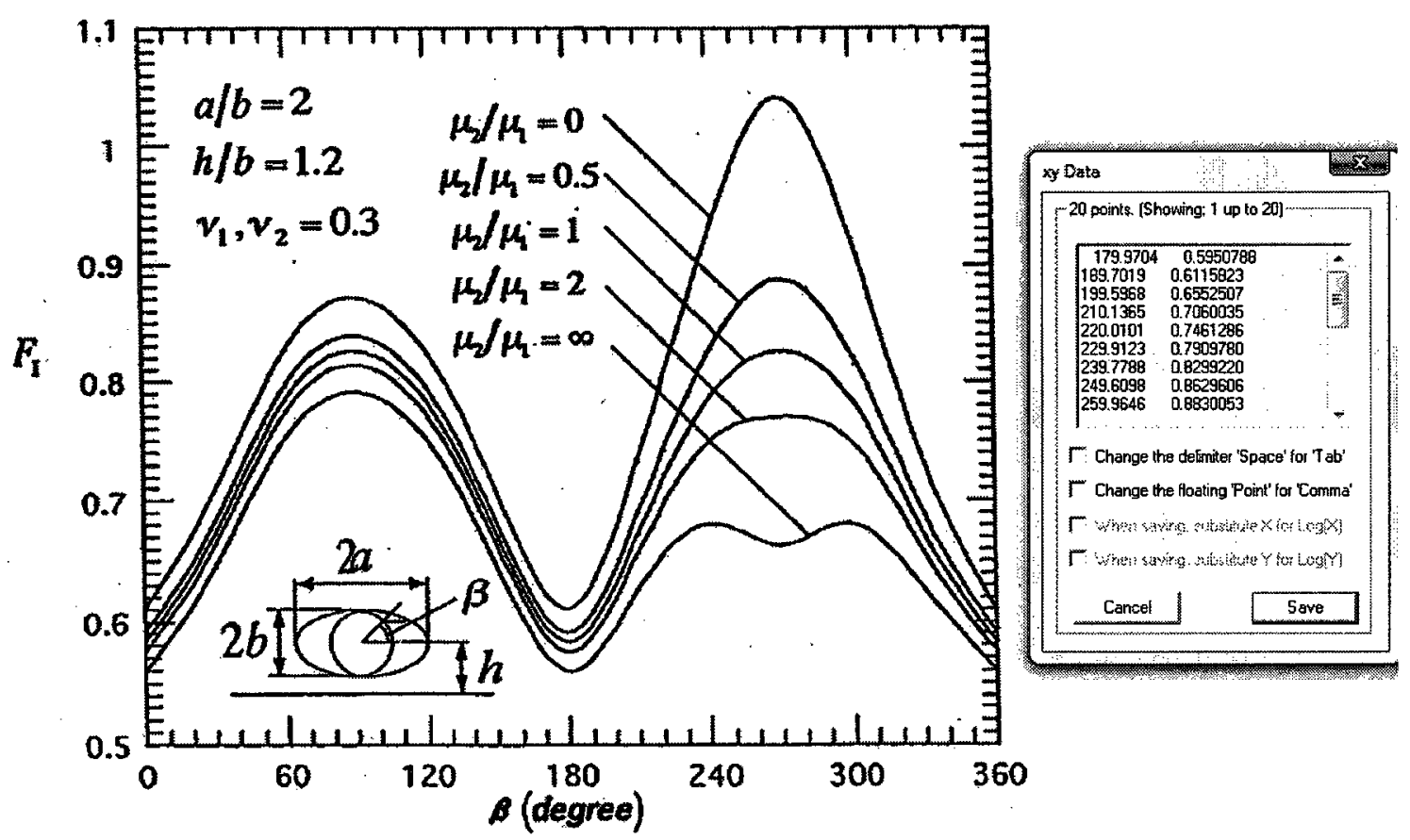

Figure 3-6. Screenshot of xyExtract software with the graph from Noda et al. [58].

A summary of the collected $F_{I}$ values corresponding to the cases $\frac{\mu_{s}}{\mu_{c}}=0.5$ and $\frac{\mu_{s}}{\mu_{c}}=2$ at $h / a=1.2, a / c=0.5$ for an angle $\pi<\xi<2 \pi$ (including the $F_{I}$ value corresponding to point $A$ mentioned in Table 3-1) is provided in Table 3-4. It is noticed that the $F_{I}$ value at point $A$ (corresponding to $\xi=\frac{3}{2} \pi$ ) extracted with the xyExtract software is slightly higher than the one reported by Noda et al. However, the difference between these values is less than $1 \%$ which is acceptable in engineering sense. 
Table 3-4. A summary of $F_{I}$ values corresponding to the cases $\mu_{s} / \mu_{c}=0.5$

and $\mu_{s} / \mu_{c}=2$ at $h / a=1.2, a / c=0.5$.

\begin{tabular}{|c|c|c|c|c|c|}
\hline$h / a$ & $a / c$ & $\mu_{s} / \mu_{c}$ & Angle $\xi(\mathrm{rad})$ & Angle $\xi(\mathrm{deg})$ & $F_{I}$ \\
\hline \multirow{19}{*}{$\frac{h}{a}=1.2$} & \multirow{19}{*}{$\frac{a}{c}=0.5$} & \multirow{19}{*}{$\frac{\mu_{s}}{\mu_{c}}=0.5$} & 3.14159 & 180 & 0.595 \\
\hline & & & 3.3161228 & 190 & 0.612 \\
\hline & & & 3.4906556 & 200 & 0.655 \\
\hline & & & 3.6651883 & 210 & 0.706 \\
\hline & & & 3.8397211 & 220 & 0.746 \\
\hline & & & 4.0142539 & 230 & 0.791 \\
\hline & & & 4.1887867 & 240 & 0.830 \\
\hline & & & 4.3633194 & 250 & 0.863 \\
\hline & & & 4.5378522 & 260 & 0.883 \\
\hline & & & 4.712385 & 270 & 0.889 \\
\hline & & & 4.8869178 & 280 & 0.882 \\
\hline & & & 5.0614506 & 290 & 0.856 \\
\hline & & & 5.2359833 & 300 & 0.818 \\
\hline & & & 5.4105161 & 310 & 0.779 \\
\hline & & & 5.5850489 & 320 & 0.736 \\
\hline & & & 5.7595817 & 330 & 0.688 \\
\hline & & & 5.9341144 & 340 & 0.650 \\
\hline & & & 6.1086472 & 350 & 0.621 \\
\hline & & & 6.28318 & 360 & 0.597 \\
\hline \multirow{19}{*}{$\frac{h}{a}=1.2$} & \multirow{19}{*}{$\frac{a}{c}=0.5$} & \multirow{19}{*}{$\frac{\mu_{s}}{\mu_{c}}=2$} & 3.14159 & 180 & 0.577 \\
\hline & & & 3.3161228 & 190 & 0.594 \\
\hline & & & 3.4906556 & 200 & 0.623 \\
\hline & & & 3.6651883 & 210 & 0.669 \\
\hline & & & 3.8397211 & 220 & 0.704 \\
\hline & & & 4.0142539 & 230 & 0.738 \\
\hline & & & 4.1887867 & 240 & 0.756 \\
\hline & & & 4.3633194 & 250 & 0.770 \\
\hline & & & 4.5378522 & 260 & 0.773 \\
\hline & & & 4.712385 & 270 & 0.775 \\
\hline & & & 4.8869178 & 280 & 0.774 \\
\hline & & & 5.0614506 & 290 & 0.768 \\
\hline & & & 5.2359833 & 300 & 0.752 \\
\hline & & & 5.4105161 & 310 & 0.728 \\
\hline & & & 5.5850489 & 320 & 0.695 \\
\hline & & & 5.7595817 & 330 & 0.658 \\
\hline & & & 5.9341144 & 340 & 0.632 \\
\hline & & & 6.1086472 & 350 & 0.602 \\
\hline & & & 6.28318 & 360 & 0.581 \\
\hline
\end{tabular}


Again, to consolidate the effect of the crack aspect ratio and to single out the effect of the interface, as we did previously, the angular interface correction factor $Y_{I a}$ was normalized with the elliptical shape factor $\sqrt{Q}$. It was also normalized with an angular function $\left(\sin ^{2} \xi+\left(\frac{a}{c}\right)^{2} \cos ^{2} \xi\right)^{1 / 4}$ (by comparison with Eqs. (3-19) and (3-22)), that is $Y_{I a}$ was multiplied by $1 / \sqrt{Q}$ and $\left(\sin ^{2} \xi+\left(\frac{a}{c}\right)^{2} \cos ^{2} \xi\right)^{1 / 4}$. Then $Y_{I n}$ was defined as normalized $Y_{I a}$, i.e., $Y_{I n}=Y_{I a} 1 / \sqrt{Q}\left(\sin ^{2} \xi+\left(\frac{a}{c}\right)^{2} \cos ^{2} \xi\right)^{1 / 4}$. A summary of normalized $Y_{I a}$ values, i.e., $Y_{I n}$ values, corresponding to the cases $\frac{\mu_{s}}{\mu_{c}}=0.5$ and $\frac{\mu_{s}}{\mu_{c}}=2$ at $h / a=1.2, a / c=0.5$ is provided in Table 3-5. 
Table 3-5. A summary of $Y_{I n}$ values corresponding to the cases $\mu_{s} / \mu_{c}=0.5$ and

$$
\mu_{s} / \mu_{c}=2 \text { at } h / a=1.2, a / c=0.5 \text {. }
$$

\begin{tabular}{|c|c|c|c|c|c|}
\hline$h / a$ & $a / c$ & $\mu_{s} / \mu_{c}$ & Angle $\xi(\mathrm{rad})$ & Angle $\xi$ (deg) & $Y_{I n}$ \\
\hline \multirow{19}{*}{$\frac{h}{a}=1.2$} & \multirow{19}{*}{$\frac{a}{c}=0.5$} & \multirow{19}{*}{$\frac{\mu_{s}}{\mu_{c}}=0.5$} & 3.1415900 & 180 & 0.584 \\
\hline & & & 3.3161228 & 190 & 0.600 \\
\hline & & & 3.4906556 & 200 & 0.643 \\
\hline & & & 3.6651883 & 210 & 0.697 \\
\hline & & & 3.8397211 & 220 & 0.754 \\
\hline & & & 4.0142539 & 230 & 0.805 \\
\hline & & & 4.1887867 & 240 & 0.848 \\
\hline & & & 4.3633194 & 250 & 0.880 \\
\hline & & & 4.5378522 & 260 & 0.899 \\
\hline & & & 4.7123850 & 270 & 0.906 \\
\hline & & & 4.8869178 & 280 & 0.899 \\
\hline & & & 5.0614506 & 290 & 0.880 \\
\hline & & & 5.2359833 & 300 & 0.848 \\
\hline & & & 5.4105161 & 310 & 0.805 \\
\hline & & & 5.5850489 & 320 & 0.754 \\
\hline & & & 5.7595817 & 330 & 0.697 \\
\hline & & & 5.9341144 & 340 & 0.643 \\
\hline & & & 6.1086472 & 350 & 0.600 \\
\hline & & & 6.2831800 & 360 & 0.584 \\
\hline \multirow{19}{*}{$\frac{h}{a}=1.2$} & \multirow{19}{*}{$\frac{a}{c}=0.5$} & \multirow{19}{*}{$\frac{\mu_{s}}{\mu_{c}}=2$} & 3.1415900 & 180 & 0.584 \\
\hline & & & 3.3161228 & 190 & 0.593 \\
\hline & & & 3.4906556 & 200 & 0.617 \\
\hline & & & 3.6651883 & 210 & 0.647 \\
\hline & & & 3.8397211 & 220 & 0.678 \\
\hline & & & 4.0142539 & 230 & 0.704 \\
\hline & & & 4.1887867 & 240 & 0.726 \\
\hline & & & 4.3633194 & 250 & 0.740 \\
\hline & & & 4.5378522 & 260 & 0.749 \\
\hline & & & 4.7123850 & 270 & 0.753 \\
\hline & & & 4.8869178 & 280 & 0.749 \\
\hline & & & 5.0614506 & 290 & 0.740 \\
\hline & & & 5.2359833 & 300 & 0.726 \\
\hline & & & 5.4105161 & 310 & 0.704 \\
\hline & & & 5.5850489 & 320 & 0.678 \\
\hline & & & 5.7595817 & 330 & 0.647 \\
\hline & & & 5.9341144 & 340 & 0.617 \\
\hline & & & 6.1086472 & 350 & 0.593 \\
\hline & & & 6.2831800 & 360 & 0.584 \\
\hline
\end{tabular}


The results from Table 3-4 and Table 3-5 are compared in Figure 3-7.

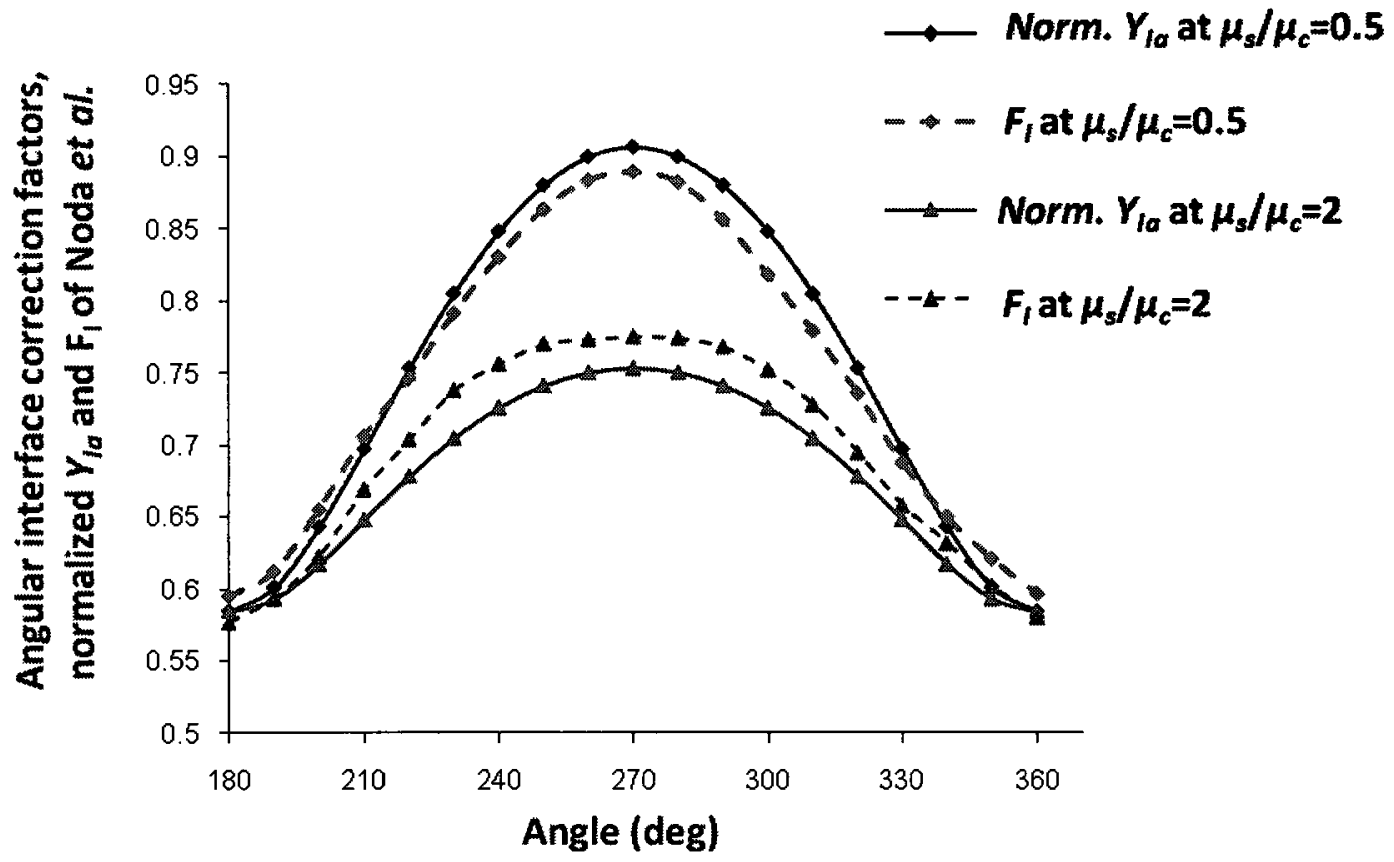

Figure 3-7. Comparison of the normalized angular interface correction factor $Y_{I a}$ to $F_{I}$ of Noda et al.

A percent error of the normalized $Y_{I a}$ (i.e., $Y_{I n}$ ) was calculated utilizing the following equation:

$\Delta=\frac{\left|F_{I}-Y_{I n}\right|}{\left|Y_{I n}\right|} \times 100 \%$

Summaries of the percent error values corresponding to the cases $\frac{\mu_{s}}{\mu_{c}}=0.5, \frac{h}{a}=1.2$, $\frac{a}{c}=0.5$ and $\frac{\mu_{s}}{\mu_{c}}=2, \frac{h}{a}=1.2$, and $\frac{a}{c}=0.5$ are presented in Table 3-6. 
Table 3-6. Percent error (\%) between $Y_{I n}$ and $F_{I}$ at $\mu_{s} / \mu_{c}=0.5, h / a=1.2$, $a / c=0.5 ;$ and at $\mu_{s} / \mu_{c}=2, h / a=1.2, a / c=0.5$.

\begin{tabular}{|c|c|c|c|c|c|c|}
\hline$h / a$ & $a / c$ & $\mu_{s} / \mu_{c}$ & Angle $\xi$ (deg) & $Y_{I n}$ & $F_{I}$ & $\Delta(\%)$ \\
\hline \multirow{19}{*}{$\frac{h}{a}=1.2$} & \multirow{19}{*}{$\frac{a}{c}=0.5$} & \multirow{19}{*}{$\frac{\mu_{s}}{\mu_{c}}=0.5$} & 180 & 0.584 & 0.595 & 1.834 \\
\hline & & & 190 & 0.600 & 0.612 & 1.812 \\
\hline & & & 200 & 0.643 & 0.655 & 1.844 \\
\hline & & & 210 & 0.697 & 0.706 & 1.258 \\
\hline & & & 220 & 0.754 & 0.746 & 0.973 \\
\hline & & & 230 & 0.805 & 0.791 & 1.745 \\
\hline & & & 240 & 0.848 & 0.830 & 2.150 \\
\hline & & & 250 & 0.880 & 0.863 & 1.968 \\
\hline & & & 260 & 0.899 & 0.883 & 1.901 \\
\hline & & & 270 & 0.906 & 0.889 & 1.966 \\
\hline & & & 280 & 0.899 & 0.882 & 2.017 \\
\hline & & & 290 & 0.880 & 0.856 & 2.803 \\
\hline & & & 300 & 0.848 & 0.818 & 3.649 \\
\hline & & & 310 & 0.805 & 0.779 & 3.313 \\
\hline & & & 320 & 0.754 & 0.736 & 2.345 \\
\hline & & & 330 & 0.697 & 0.688 & 1.327 \\
\hline & & & 340 & 0.643 & 0.650 & 1.088 \\
\hline & & & 350 & 0.600 & 0.621 & 3.235 \\
\hline & & & 360 & 0.584 & 0.597 & 2.150 \\
\hline \multirow{19}{*}{$\frac{h}{a}=1.2$} & \multirow{19}{*}{$\frac{a}{c}=0.5$} & \multirow{19}{*}{$\frac{\mu_{s}}{\mu_{c}}=2$} & 180 & 0.584 & 0.577 & 1.242 \\
\hline & & & 190 & 0.593 & 0.594 & 0.166 \\
\hline & & & 200 & 0.617 & 0.623 & 0.977 \\
\hline & & & 210 & 0.647 & 0.669 & 3.206 \\
\hline & & & 220 & 0.678 & 0.704 & 3.699 \\
\hline & & & 230 & 0.704 & 0.738 & 4.542 \\
\hline & & & 240 & 0.726 & 0.756 & 4.022 \\
\hline & & & 250 & 0.740 & 0.770 & 3.796 \\
\hline & & & 260 & 0.749 & 0.773 & 2.991 \\
\hline & & & 270 & 0.753 & 0.775 & 2.850 \\
\hline & & & 280 & 0.749 & 0.774 & 3.117 \\
\hline & & & 290 & 0.740 & 0.768 & 3.546 \\
\hline & & & 300 & 0.726 & 0.752 & 3.511 \\
\hline & & & 310 & 0.704 & 0.728 & 3.230 \\
\hline & & & 320 & 0.678 & 0.695 & 2.452 \\
\hline & & & 330 & 0.647 & 0.658 & 1.587 \\
\hline & & & 340 & 0.617 & 0.632 & 2.386 \\
\hline & & & 350 & 0.593 & 0.602 & 1.493 \\
\hline & & & 360 & 0.584 & 0.581 & 0.544 \\
\hline
\end{tabular}


The error values of the normalized angular interface correction factor $Y_{I a}$ are within 5\% compared to the results of Noda et al., which confirms the accuracy of the proposed interface correction factor $Y_{I a}$. As a result, the angular stress intensity factor of the elliptical crack embedded in a bi-material media under remote tensile loading can be represented with an expression as follows:

$$
\begin{aligned}
& K_{I}=\sigma_{\infty} \cdot \sqrt{\frac{\pi a}{Q}} \cdot\left(\sin ^{2} \xi+\left(\frac{a}{c}\right)^{2} \cos ^{2} \xi\right)^{1 / 4} \cdot \frac{1+\frac{\mu_{s}}{\mu_{c}} \cos \left(\frac{\pi a \sin \xi}{2(a \sin \xi+h)}\right)}{\frac{\mu_{s}}{\mu_{c}}+\cos \left(\frac{\pi a \sin \xi}{2(a \sin \xi+h)}\right)} \\
& (\pi<\xi<2 \pi)
\end{aligned}
$$

The interface correction factor verified for the remote tensile loading case is by nature independent from applied loading. Therefore, it is applicable to the condition of having well-developed median cracks near a bi-material interface under a point load (which is the case of the current study). Thus, the stress intensity factor $K_{r}$ represented by Eq. (3-10 a) could be normalized using the interface correction factor $Y_{I a}$ as follows:

$$
K_{r}=Y_{I a}(\xi) \cdot \chi_{r} \cdot \beta \cdot \frac{P}{c^{3 / 2}} \cdot \Phi(\xi, k) \quad(\pi<\xi<2 \pi)
$$

where according to Eq. (3-25):

$$
Y_{I a}(\xi)=\frac{1+\frac{\mu_{s}}{\mu_{c}} \cos \left(\frac{\pi a \sin \xi}{2(a \sin \xi+h)}\right)}{\frac{\mu_{s}}{\mu_{c}}+\cos \left(\frac{\pi a \sin \xi}{2(a \sin \xi+h)}\right)}
$$

and the residual factor $\chi_{r}$ is described with Eq. (3-16) as:

$$
\chi_{r}=\frac{1}{3 \pi^{2} \cdot\left(1-2 v_{c}\right)} \cdot\left(\frac{E_{c}}{H_{m}}\right)^{1 / 2} \cdot(\cot \psi)^{2 / 3}
$$


According to Eq. (3-25), the interface correction factor $Y_{I a}(\xi)=1$ at $\xi=\pi$ and $\xi=2 \pi$. These points are located on the major axis of the ellipse at the surface trace crack tips. This manifests that the stress intensity factor evaluated at the crack tips that are far from the interface, is not influenced by the interface correction factor.

\subsubsection{Case III: $a>h_{c}$}

Finally, consider the situation when the indenter penetrates through the coating layer under the load $P$ in a depth of $d=h_{c}+d_{i n d} \cdot d_{\text {ind }}$ is the depth of the indenter penetration into the substrate, and it generates a radial/median crack of a size $c$, as shown in Figure 3-2. Further, a coating/substrate system containing a radial/median crack after a full indenter removal is considered. This implies that the elastic component of the indentation field has diminished and the residual field is the only driving force for the crack.

In this analysis, it is postulated that the coating remains well-bonded to the substrate outside the crack area. Furthermore, a segment of the system is plastically deformed by the indenter over a contact $l$ (Figure 3-2) and penetration depth $d_{\text {ind }}$ in such a way that irreversible strains associated with the indenter penetration are accommodated by an expansion of the spherical plastic zone in the coating/substrate media at a constant volume of the coating/substrate material. Under these assumptions, a deformation compatibility condition in the near field of the brittle coating/ductile substrate system is naturally imposed. The residual stress field component after indenter removal can be evaluated as follows:

I. The volumetric expansion of the coating under the Vickers indenter is of the same order as the volumetric expansion of the substrate by compatibility; 
II. The coating deforms under the indenter and expands spherically with a hydrostatic pressure $p_{c}=\left(\sigma_{x}^{c}+\sigma_{y}^{c}+\sigma_{z}^{c}\right) / 3$. Similarly, a plastically deformed substrate also expands spherically and induces a tensile hydrostatic pressure $p_{s}=\left(\sigma_{x}^{s}+\sigma_{y}^{s}+\sigma_{z}^{s}\right) / 3$ in the surrounding elastic matrix.

Assuming that $\varepsilon_{x}^{c}, \varepsilon_{y}^{c}, \varepsilon_{z}^{c}$ and $\varepsilon_{x}^{s}, \varepsilon_{y}^{s}, \varepsilon_{z}^{s}$ are the strain components associated with the coating and substrate respectively, the strain-stress relationships for the coating and substrate can be written as:

$$
\begin{aligned}
& \varepsilon_{x}^{c}=\frac{\sigma_{x}^{c}}{E_{c}}-\frac{\nu_{c}\left(\sigma_{y}^{c}+\sigma_{z}^{c}\right)}{E_{c}} \\
& \varepsilon_{y}^{c}=\frac{\sigma_{y}^{c}}{E_{c}}-\frac{\nu_{c}\left(\sigma_{z}^{c}+\sigma_{x}^{c}\right)}{E_{c}} \\
& \varepsilon_{z}^{c}=\frac{\sigma_{z}^{c}}{E_{c}}-\frac{\nu_{c}\left(\sigma_{x}^{c}+\sigma_{y}^{c}\right)}{E_{c}} \\
& \varepsilon_{x}^{s}=\frac{\sigma_{x}^{s}}{E_{s}}-\frac{\nu_{s}\left(\sigma_{y}^{s}+\sigma_{z}^{s}\right)}{E_{s}} \\
& \varepsilon_{y}^{s}=\frac{\sigma_{y}^{s}}{E_{s}}-\frac{\nu_{s}\left(\sigma_{z}^{s}+\sigma_{x}^{s}\right)}{E_{s}} \\
& \varepsilon_{z}^{s}=\frac{\sigma_{z}^{s}}{E_{s}}-\frac{\nu_{s}\left(\sigma_{x}^{s}+\sigma_{y}^{s}\right)}{E_{s}}
\end{aligned}
$$

where $\left(\sigma_{x}^{c}, \sigma_{y}^{c}, \sigma_{z}^{c}\right)$ and $\left(\sigma_{x}^{s}, \sigma_{y}^{s}, \sigma_{z}^{s}\right)$ are the stress components associated with the coating and the substrate respectively. $E_{c}, v_{c}$ and $E_{s}, v_{s}$ are the Young's moduli and Poisson's ratios corresponding to the coating and substrate materials, respectively. The summation of Eqs. (3-29) to (3-31) yields: 
$\varepsilon_{x}^{c}+\varepsilon_{y}^{c}+\varepsilon_{z}^{c}=\frac{1}{E_{c}} \cdot\left(1-2 v_{c}\right)\left(\sigma_{x}^{c}+\sigma_{y}^{c}+\sigma_{z}^{c}\right)$

The summation of Eqs. (3-32) to (3-34) gives:

$\varepsilon_{x}^{s}+\varepsilon_{y}^{s}+\varepsilon_{z}^{s}=\frac{1}{E_{s}} \cdot\left(1-2 v_{s}\right)\left(\sigma_{x}^{s}+\sigma_{y}^{s}+\sigma_{z}^{s}\right)$

The summation of the three direct co-ordinate strains $\varepsilon_{x}^{c}+\varepsilon_{y}^{c}+\varepsilon_{z}^{c}$ and $\varepsilon_{x}^{s}+\varepsilon_{y}^{s}+\varepsilon_{z}^{s}$ are volumetric strains $\varepsilon^{c}$ and $\varepsilon^{s}$ in coating and substrate, respectively. Since

$\frac{1}{3}\left(\sigma_{x}^{c}+\sigma_{y}^{c}+\sigma_{z}^{c}\right)=p_{c}$

and

$\frac{1}{3}\left(\sigma_{x}^{s}+\sigma_{y}^{s}+\sigma_{z}^{s}\right)=p_{s}$

the expressions (3-35) and (3-36) can be re-written as

$$
\begin{aligned}
& \varepsilon^{c}=\frac{3\left(1-2 v_{c}\right)}{E_{c}} \cdot p_{c} \\
& \varepsilon^{s}=\frac{3\left(1-2 v_{s}\right)}{E_{s}} \cdot p_{s}
\end{aligned}
$$

By strain compatibility,

$$
\varepsilon^{c}=\frac{3\left(1-2 v_{c}\right) p_{c}}{E_{c}}=\varepsilon^{s}=\frac{3\left(1-2 v_{s}\right) p_{s}}{E_{s}}
$$

Furthermore, the average pressure induced on the deformed segment of the coating/substrate system is

$$
\begin{aligned}
& p_{c} A_{c}+p_{s} A_{s}=p_{a v} \cdot A_{\text {total }} \\
& A_{c}+A_{s}=A_{\text {total }}
\end{aligned}
$$


where $A_{c}$ and $A_{s}$ are the areas of the plastic zone in the coating portion and in the substrate portion, respectively. $A_{\text {total }}$ is the total area of the plastic zone surface; $p_{a v}$ is the average pressure distributed over this area. By utilizing composite volume fraction analogy, the volume fractions of the plastic zone under pressure in the coating/substrate system are respectively, as follows:

$$
\begin{gathered}
f_{c}=\frac{A_{c}}{A_{\text {total }}} \\
f_{s}=\frac{A_{s}}{A_{\text {total }}}
\end{gathered}
$$

Hence, the expression (3-42) can be rewritten as

$$
p_{c} f_{c}+p_{s} f_{s}=p_{a v}
$$

Substituting Eqs. (3-39) and (3-40) in Eq. (3-46) gives

$$
\left(\frac{E_{s}}{3\left(1-2 v_{s}\right)} f_{s}+\frac{E_{c}}{3\left(1-2 v_{c}\right)} f_{c}\right) \varepsilon^{s}=p_{a v}
$$

On the other hand, based on assumptions I and II, the relative change in volume of the expanding plastic zone is determined, similarly to Eq. (3-1), as

$$
\frac{\delta V}{V} \approx \varepsilon^{c}{ }_{x}+\varepsilon^{c}{ }_{y}+\varepsilon_{z}^{c}=\varepsilon_{x}^{s}+\varepsilon^{s}{ }_{y}+\varepsilon_{z}{ }^{s}=\frac{l^{3} \cdot \cot \psi}{b^{3}}
$$

The considered segment can be restored to its original characteristic $b$ (Figure 3-2) by imposed external hydrostatic compression across the outer boundaries [18]:

$$
p_{a v} \approx K_{m} \cdot \frac{\delta V}{V}
$$


where $K_{m}$ is the bulk modulus of the brittle coating/ductile substrate system, which can be defined based on Eq. (3-47) and provided that Poisson's ratios $v_{s} \approx v_{c} \approx v_{m}$, as follows:

$$
K_{m}=\frac{E_{m}}{3\left(1-2 v_{m}\right)} \approx \frac{\left(E_{s} f_{s}+E_{c} f_{c}\right)}{3\left(1-2 v_{m}\right)}
$$

where $E_{m}$ and $v_{m}$ are Young's modulus and Poisson's ratio of the coating/substrate system respectively. Then, following the procedures outlined in Eq. (3-1) to Eq. (3-6), we can obtain an expression for the force exuded by the plastic field approximated as a point force on the crack:

$$
P_{r} \approx p_{a v} \cdot b^{2}=\frac{E_{m}}{3\left(1-2 v_{m}\right)} \cdot\left(\frac{l}{b}\right)^{3} \cdot \cot \psi \cdot b^{2}
$$

By analogy to an expanded cavity in an infinite isotropic elastic-plastic matrix [11],

$$
\frac{b}{l}=\left(\frac{E_{m}}{H}\right)^{m} \cdot \cot \psi^{1 / 3}
$$

where $m \approx 1 / 2[11]$.

Using Eq. (3-5) to express Vickers hardness $H$ of the coating/substrate system combined with Eq. (3-52), Eq. (3-51) reduces to:

$$
P_{r}=\frac{P}{3\left(1-2 v_{m}\right)} \cdot\left(\frac{E_{m}}{H}\right)^{1 / 2} \cdot(\cot \psi)^{2 / 3}
$$

Substituting Eq. (3-53) in the expression of the stress intensity factor for an elliptical crack under a point force applied in the center of the crack yields an expression described with Eq. (3-10 a), i.e., 


$$
K_{r}=\chi_{r} \cdot \beta \cdot \frac{P}{c^{3 / 2}} \cdot \Phi(\varphi, k)
$$

where

$$
\chi_{r} \approx \frac{1}{3 \pi^{2} \cdot\left(1-2 v_{m}\right)} \cdot\left(\frac{E_{m}}{H}\right)^{1 / 2} \cdot(\cot \psi)^{2 / 3}
$$

\subsection{Closing Remarks}

Chapter 3 presents the development procedures of a model for fracture toughness assessment of brittle coating/ductile substrate systems in terms of linear elastic-plastic contact fracture mechanics principles. Radial/median cracks of an arbitrary depth in the coating are considered, with three scenarios involved. They are (1): the crack depth is less than $7 \%$ of the coating thickness; (2) the crack lies in-between $7 \%$ of the coating thickness and the total coating thickness, and (3) the crack penetrates the substrate. The solutions for each scenario are derived.

To incorporate the effect of the presence of the coating/substrate interface, an improved interface correction factor which highlights the effect of the distance of the crack to the interface, based on the work of Noda et al., is proposed and is further formalized in light of engineering judgment. For comparison, the proposed equation for the improved interface correction factor is normalized to be consistent with the interface correction factor provided by Noda $e t a l$. It is demonstrated that the values derived from the normalized equation are good fits to the data of the interface correction factor provided by Noda et al. However, this new interface correction factor is a compact analytical expression which can be easily utilized in engineering applications provided that shear moduli of a coating/substrate system are known. It offers a distinct advantage 
over the boundary factor provided by Noda et al. which is represented with a cumbersome set of expressions requiring complex numerical evaluation. Another advantage is that the proposed interface correction factor explicitly showcases an interface influence as a function of the distance between the crack and the interface as opposed to the set of equations derived by Noda et al.

Furthermore, the proposed equation for the stress intensity factor of the elliptical crack in the coating/substrate system takes into account the effect of the crack geometry, substrate material properties and the presence of the coating/substrate interface.

The proposed model needs to be further verified. For this purpose, a series of Vickers indentation tests were conducted. In the meanwhile, optical microscopy and focused ion beam techniques were utilized to confirm the assumptions made for the model development in Chapter 3. The details of the performed experimental work are described in Chapter 4. 


\section{Chapter 4. Experimental Details}

\subsection{Objectives of the Experiments}

Chapter 4 describes the experimental work conducted to address multiple objectives in this research. The first objective was to validate the key assumptions made in development of the fracture mechanics model in Chapter 3. As shown previously, the first assumption that requires validation is that a well-developed crack/fracture pattern is radial/median. The second assumption is that a well-developed radial/median crack is elliptical in shape. To confirm these assumptions, a series of Vickers indentation tests at different indentation loads were performed. Furthermore, the selected indentation marks in the coating specimen were dissected normally to the coating surface utilizing a focused ion beam (FIB) technique to examine the crack profile.

The second objective of the experimental work was to collect experimental data that could subsequently be used in Chapter 5 to validate the fracture toughness model proposed in Chapter 3. Thus, a scanning electron microscope was utilized to determine an average coating thickness. Utilizing optical microscopy, the radial crack lengths and indentation mark diagonals at the corresponding Vickers indentation loads were collected from the post-indentation tests. The coating and substrate hardness values were also determined under the Vickers indentation.

\subsection{Coating Specimen Preparation}

\subsubsection{Coating Deposition}

A hard tungsten carbide coating $\mathrm{WC} / 10 \mathrm{Co} / 4 \mathrm{Cr}$ deposited on a 1080 low carbon steel substrate specimens was chosen for the experimental study. $\mathrm{WC} / 10 \mathrm{Co} / 4 \mathrm{Cr}$ is a 
brittle material and 1080 low carbon steel is ductile. The values of the Young's moduli of the coating and substrate materials were adopted from the previous research work [60] as follows: Young's modulus $E_{c}=310 \mathrm{GPa}$ of the WC/10Co/4Cr coating and Young's modulus $E_{s}=250 \mathrm{GPa}$ of the 1080 low carbon steel.

The coating specimen of $\mathrm{WC} / 10 \mathrm{Co} / 4 \mathrm{Cr}$ was fabricated with the Jet Kote High Velocity Oxygen Fuel (HVOF) system at Deloro Stellite Group. In the High Velocity Oxygen Fuel (HVOF) process, thoroughly mixed powders were axially introduced into the chamber with the gas flame which was constantly burning under high pressure [60]. The exhaust gas was produced by internal combustion of oxygen and fuel gas, and fed through an expansion nozzle. The exhaust gas travelling through the expansion nozzle was accelerated to an initial velocity of $1350 \mathrm{~m} / \mathrm{s}$; the powder particles were heated to a temperature of $3000^{\circ} \mathrm{C}$ and directly propelled with kinetic energy onto the work piece surface without a bond coat. The resulting coating was very dense (porosity was less than $1 \%$ ); the powder particle size was approximately $40 \sim 100$ microns.

\subsubsection{Specimens for Coating Thickness Measurement}

The coating specimen was sectioned with a Diamond Cut Saw machine at cutting speed of $1000 \mathrm{rpm}$; the dimensions of the resulting smaller pieces were $13 \times 15 \mathrm{~mm}$. Additionally, one of the smaller specimens was further cut into half, which was used to estimate its coating thickness before and after grinding and polishing. All specimens were cleaned for approximately 10 minutes with acetone or methanol solution in an ultrasonic cleaner.

To prepare a specimen for an average thickness measurement of the coating, firstly, the unpolished $\mathrm{WC} / 10 \mathrm{Co} / 4 \mathrm{Cr}$ coating was cold mounted in a mounting cup with 
its cross-section exposed as the top surface. 10 parts of the Hysol epoxy resin and 1 part of the Hysol hardener HD3404 were mixed together to create $20 \mathrm{~g}$ of uniform mixture. The specimen was placed in a holder and then in a holding cup; the mixture was poured over it and the holding cup was placed in a vacuum chamber (Struers Epovoc). The cold mounted specimen was left in a vacuum chamber for 24 hours to complete the cycle. Following that, the prepared specimen (its exposed side) was then ground and polished with grit papers varying from $\# 180$ to $\# 1200$ on an automatic grinding machine Ecomet IV, Buehler with a power head Euromet I. The same procedure was followed in preparation of the specimen for measuring the average coating thickness after the surface was polished.

In parallel, several coating specimens were prepared to undergo Vickers indentation tests. To accomplish that, the coating specimens had to be ground and polished to provide for a smoother surface that allowed for post-indentation examination. To facilitate the manual grinding and polishing process of these specimens, the holding packs were manufactured. For this purpose, a fully automatic, hot mounting press LaboPress-3 (Struers) was used. Three powder packs were placed on the upper ram which was then lowered into a cylinder, closed with a top closure and left for a 9 minute cycle. The 9 minute cycle consisted of 6 minute heating time and 3 minute cooling time; the heating temperature was up to $180^{\circ} \mathrm{C}$. The specimens were mounted on the previously prepared packs; the coating surfaces of the specimens were then manually ground and polished with grit papers varying from $\# 180$ to $\# 1200$ and Texmet 1500 cloth with 3 micron abrasive fluid. The ground and polished specimens were finally cleaned 
once again inside the ultrasonic cleaner and stored in clean plastic packages for future Vickers indentation tests.

\subsubsection{Coating Thickness Measurement}

The average coating thickness measurements were taken with a Hitachi Model S-570 scanning electronic microscope (SEM). The SEM is a type of electron microscope that images the sample surface by scanning it with a high-energy beam of electrons in a raster scan pattern. The electrons interface with the atoms that make up the sample producing signals, and these signals contain information about the sample's surface topography and composition. The SEM can produce very high-resolution images of sample surfaces up to 1 to $5 \mathrm{~nm}$ in size. To use the SEM, the specimens must have an electrically conductive surface to prevent the accumulation of electrostatic charge at the surface [61].

The coating thickness measurements were performed on the cross-section of the coating specimen before and after polishing. The SEM images are presented in Figure 4-1. The images show that the deposited coating is not uniform in thickness. The coating thickness after polishing ranges from $38.11 \mu \mathrm{m}$ to $52.16 \mu \mathrm{m}$ yielding to the average thickness of $45.3 \mu \mathrm{m}$. The as-deposited average coating thickness (before polishing) is about $150 \mu \mathrm{m}$. 


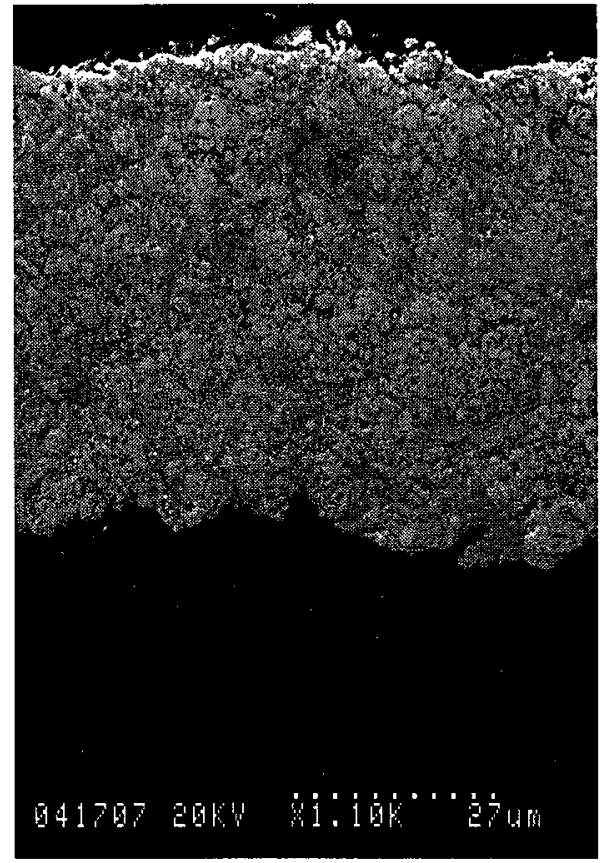

(a)

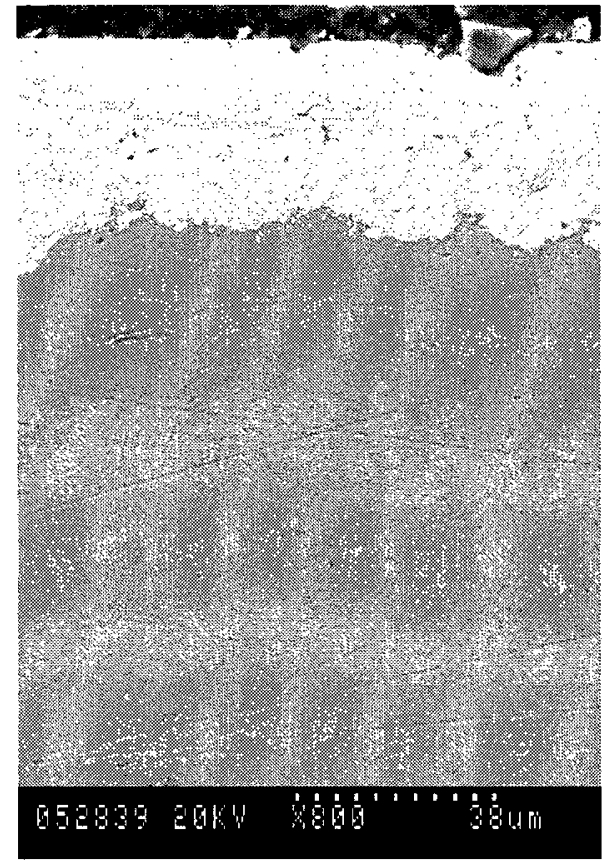

(b)

Figure 4-1. SEM images of the cross-section of the WC/10Co/4Cr coating/1080 low carbon steel substrate specimen: (a) before polishing and (b) after polishing.

\subsection{Vickers Indentation Tests}

\subsubsection{Testing Methods}

The polished coating specimens were subjected to the Vickers indentation tests at different loads such as: $P=196 \mathrm{~N}, P=294 \mathrm{~N}, P=392 \mathrm{~N}, P=490 \mathrm{~N}$ and $P=720 \mathrm{~N}$. The angle of the Vickers indenter is $136^{\circ}$. To ensure non-interference of the residual fields generated by the Vickers indenter, the indentation marks were made at least 2.5 times Vickers diagonals away from each other and the specimen edges; the angle between the indenter and specimen surface was within $2^{\circ}$ of the perpendicular [62]. At least three indentation marks were generated at each load. 


\subsubsection{Post-Indentation Examination}

The indented coating surfaces were examined with an optical microscope Olympus PMG-3. A Quartz PCI Version 5 image acquisition and archiving software package allowed analyzing and gathering of data in regard to radial crack lengths and indentation mark diagonals. The typical fracture patterns observed after the Vickers indentation tests on the $\mathrm{WC} / 10 \mathrm{Co} / 4 \mathrm{Cr}$ coating/1080 low carbon steel substrate specimen are shown in Figure 4-2 with the measurements of the indentation mark diagonals and radial crack lengths. The radial cracks were initiated at the corners of the indentation marks and were measured from the center of the indentation marks to the radial crack ends.

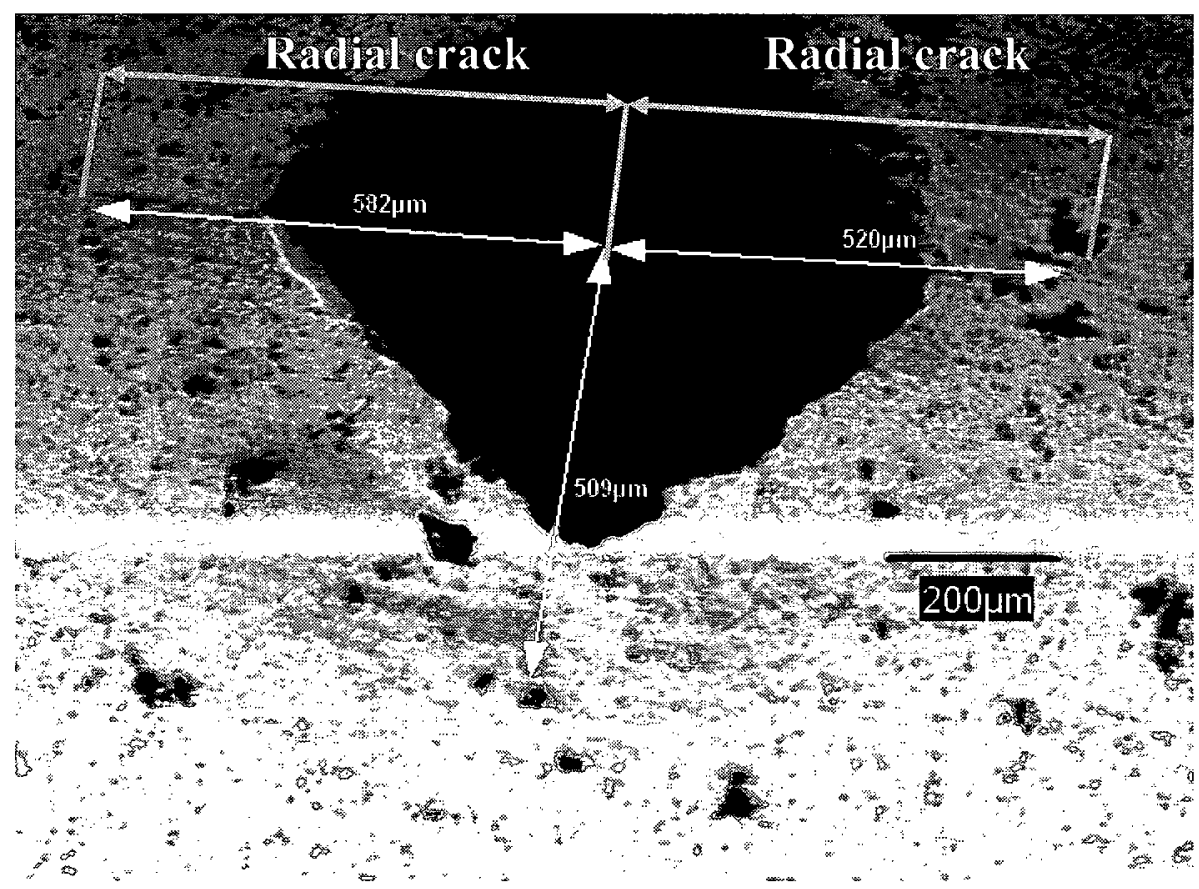

(a) Radial crack lengths at $\mathbb{P}=196 \mathbb{N}$. 


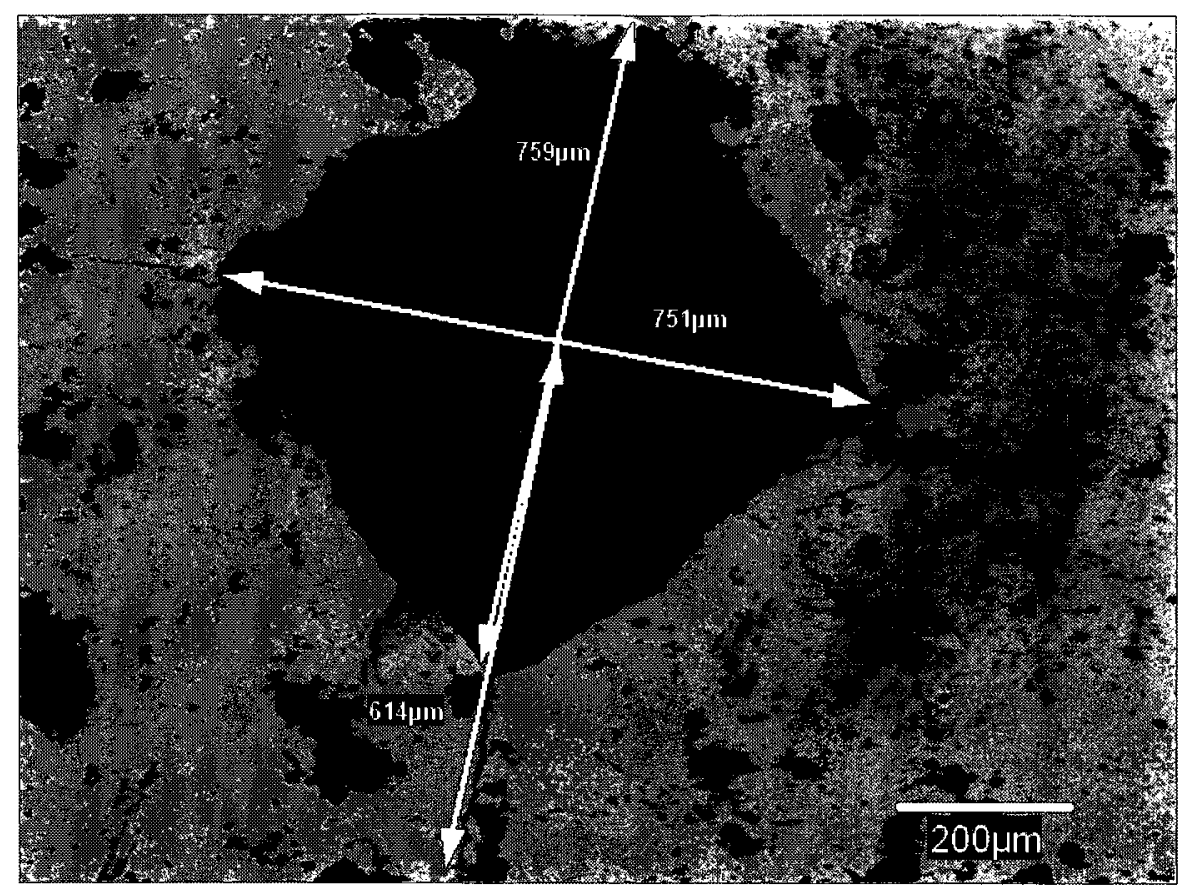

(b) Indentation mark diagonals at $P=196 \mathbb{N}$.

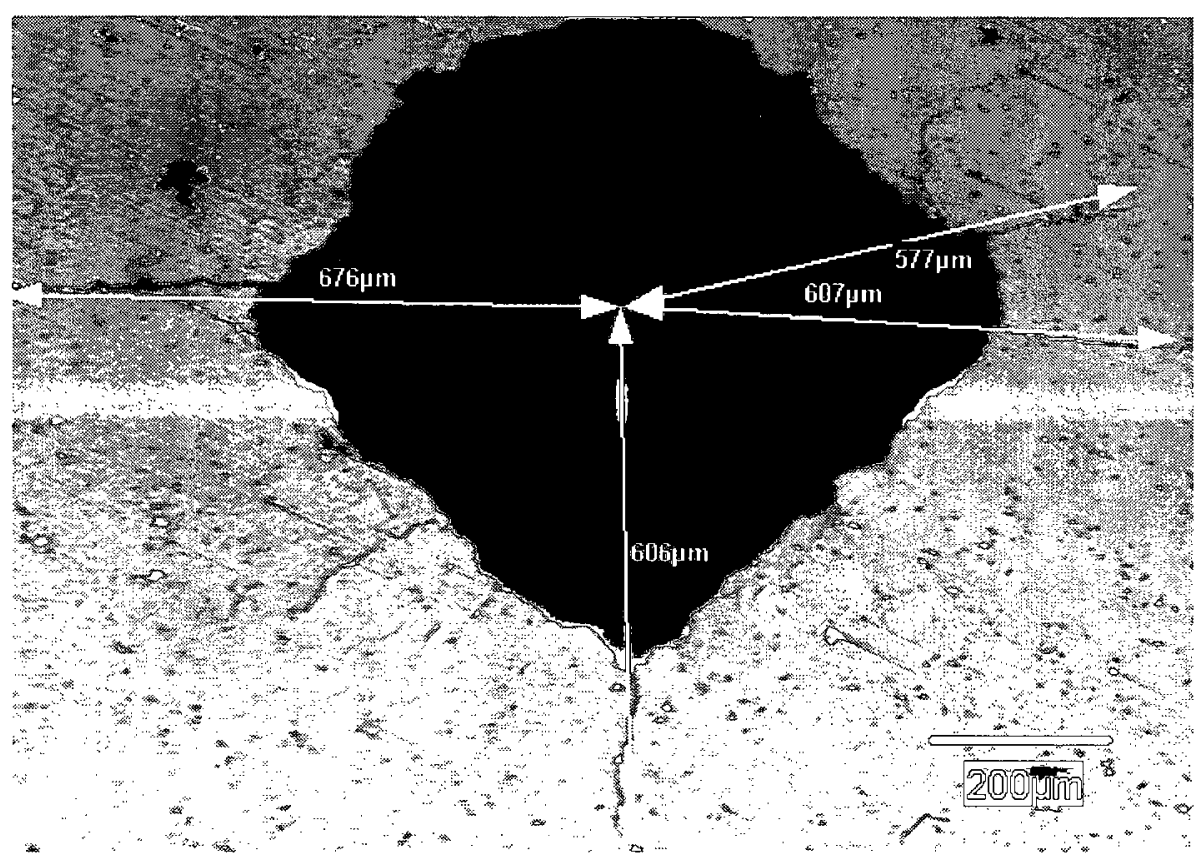

(c) Radial crack lengths at $\mathbb{P}=294 \mathbb{N}$. 


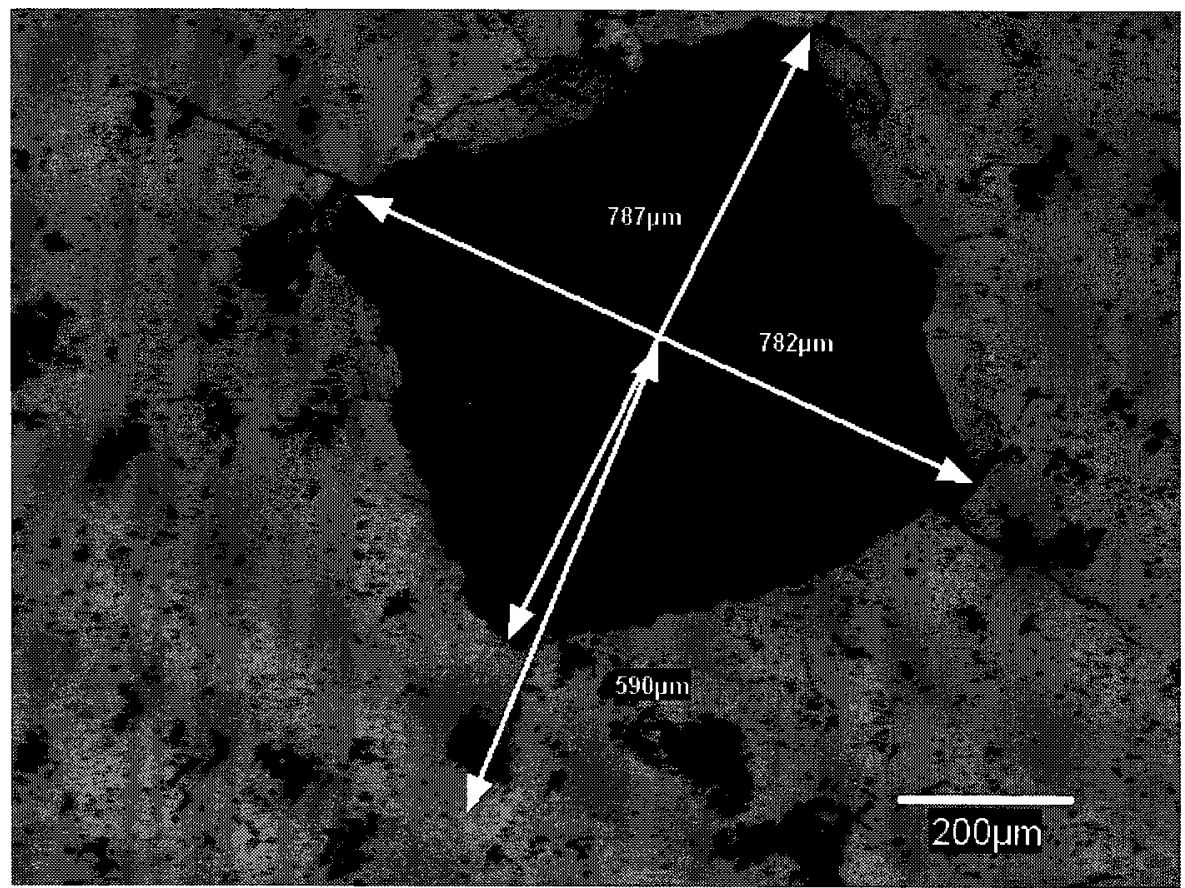

(d) Indentation mark diagonals at $P=294 \mathrm{~N}$.

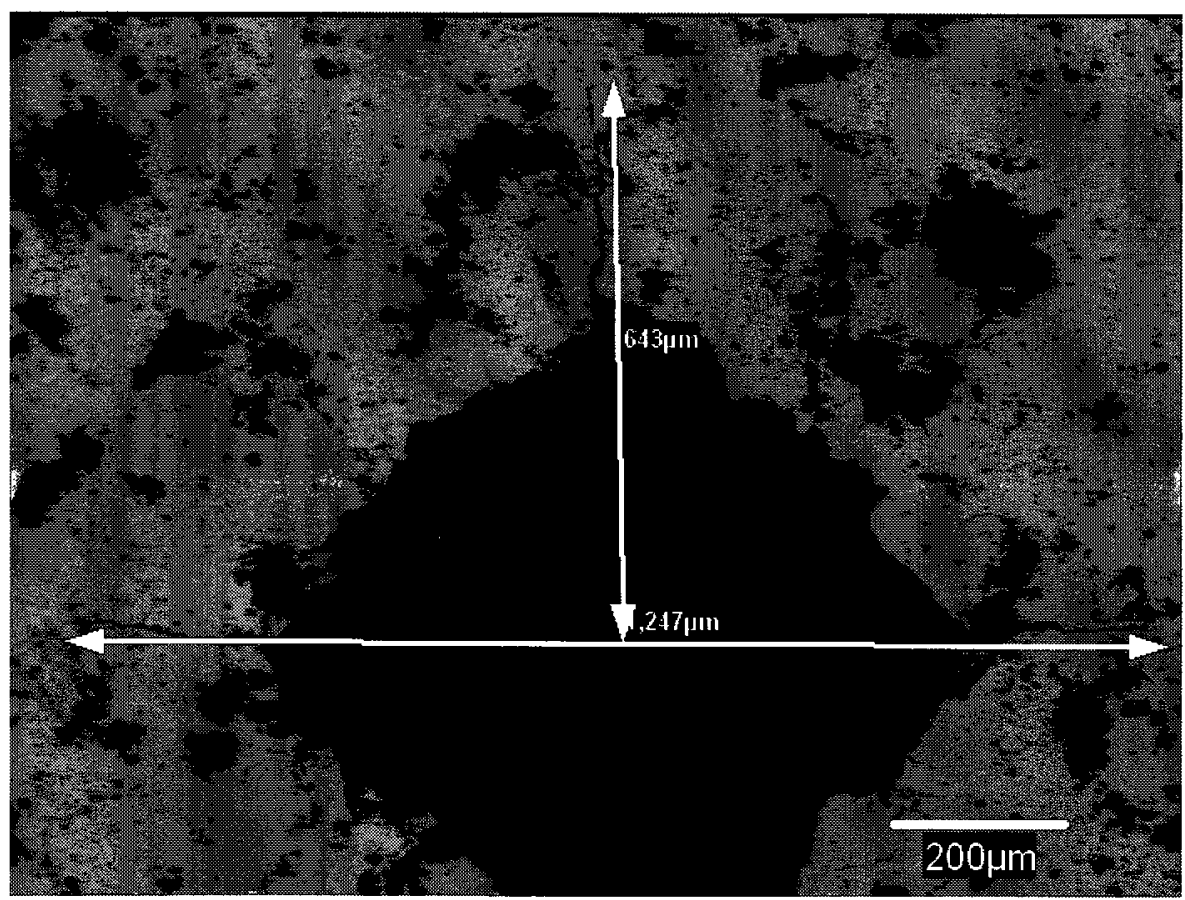

(e) Radial crack lengths at $P=392 \mathrm{~N}$. 


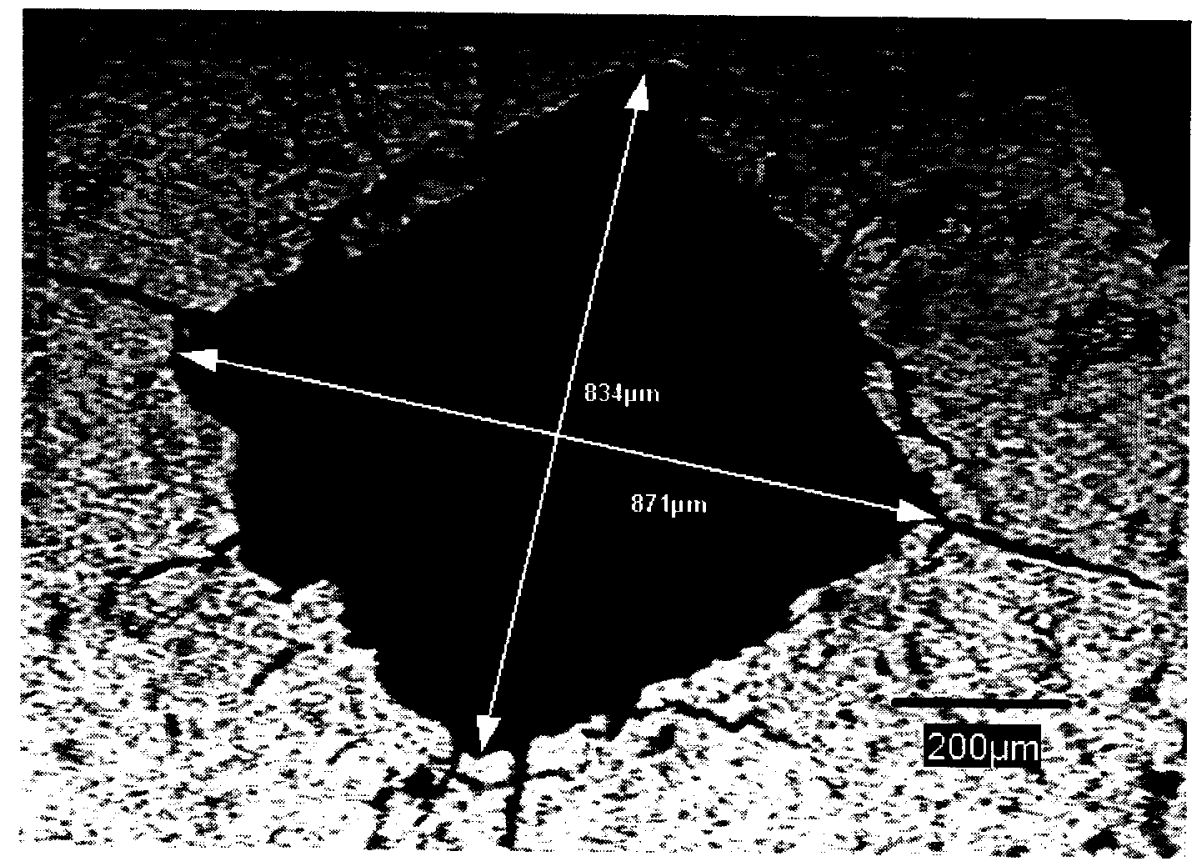

(f) Indentation mark diagonals at $P=392 \mathrm{~N}$.

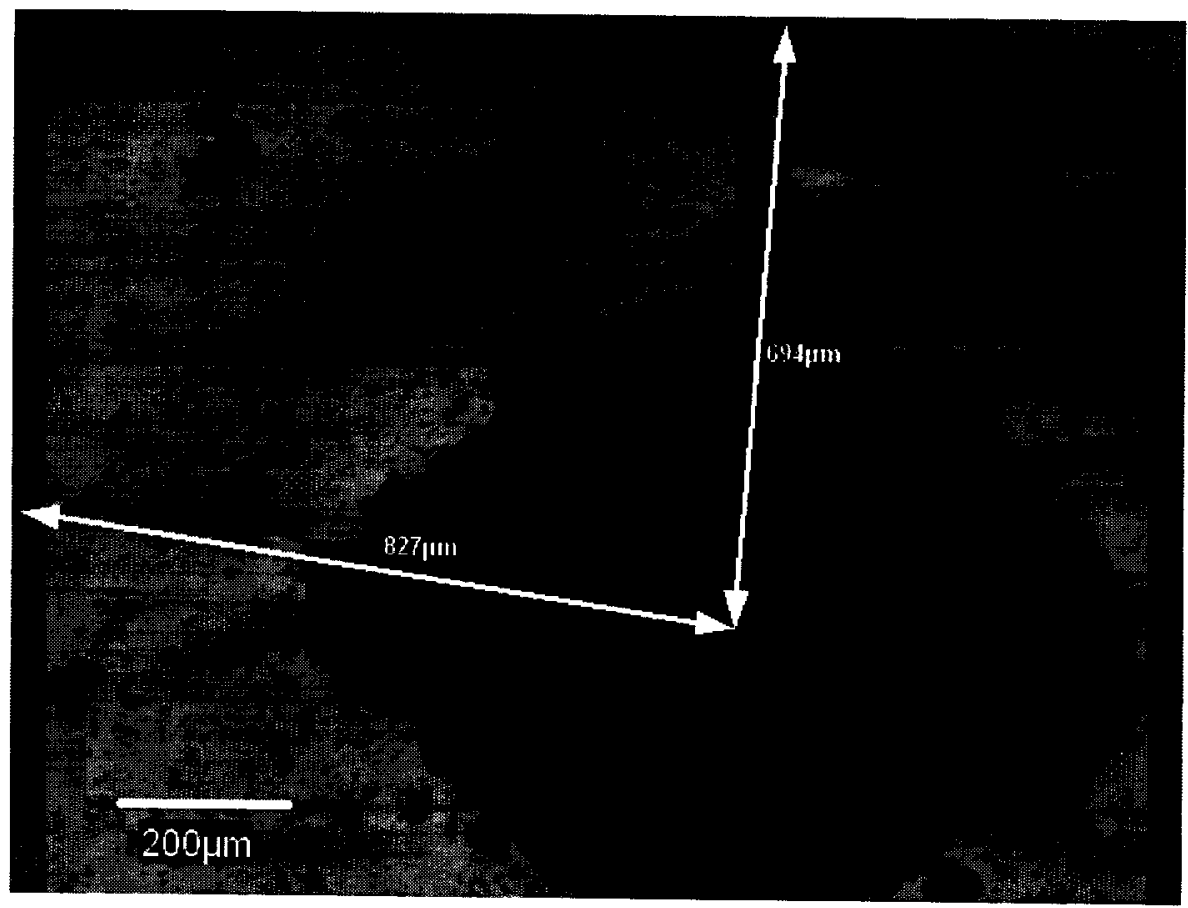

(g) Radial crack lengths at $P=490 \mathrm{~N}$. 


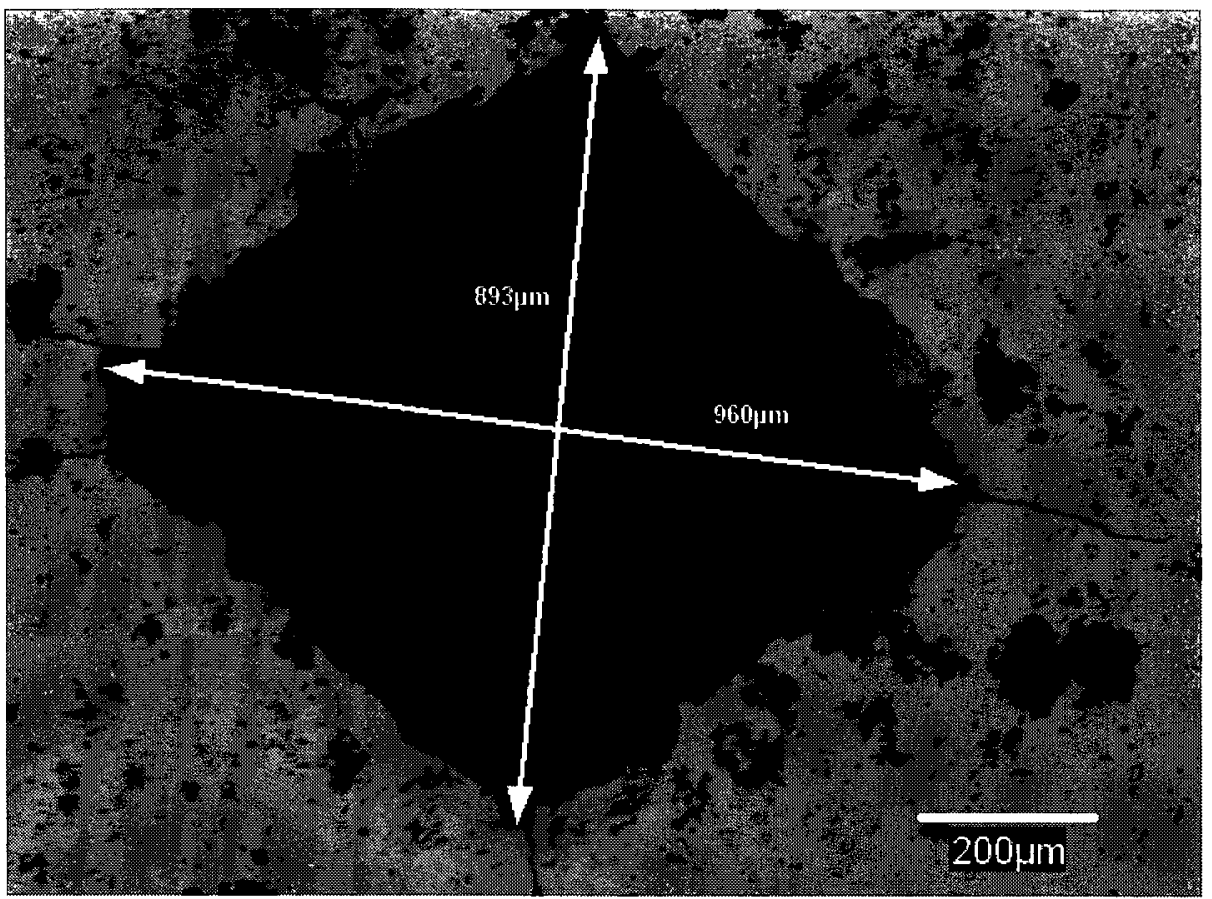

(h) Indentation mark diagonals at $P=490 \mathrm{~N}$.

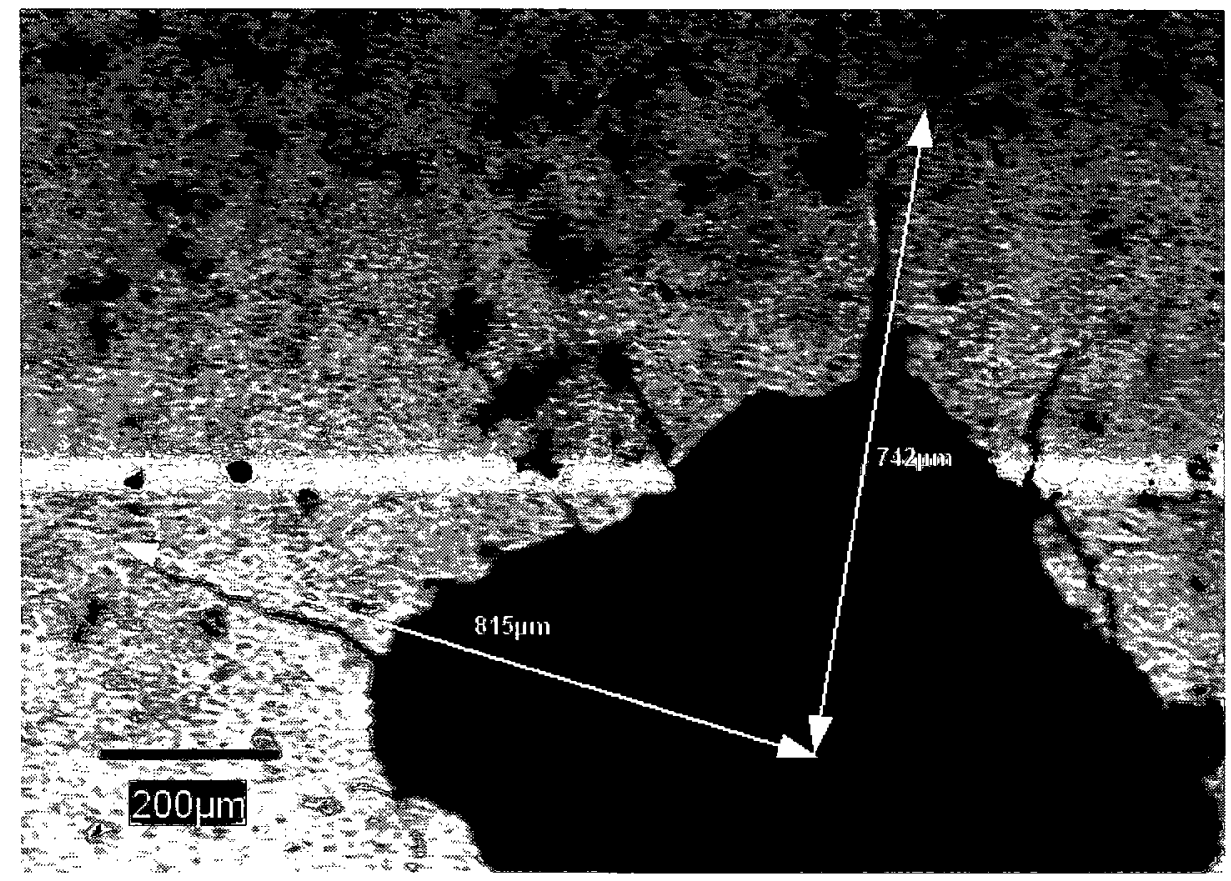

(ii) Radial crack lengths at $P=720 \mathbb{N}$. 


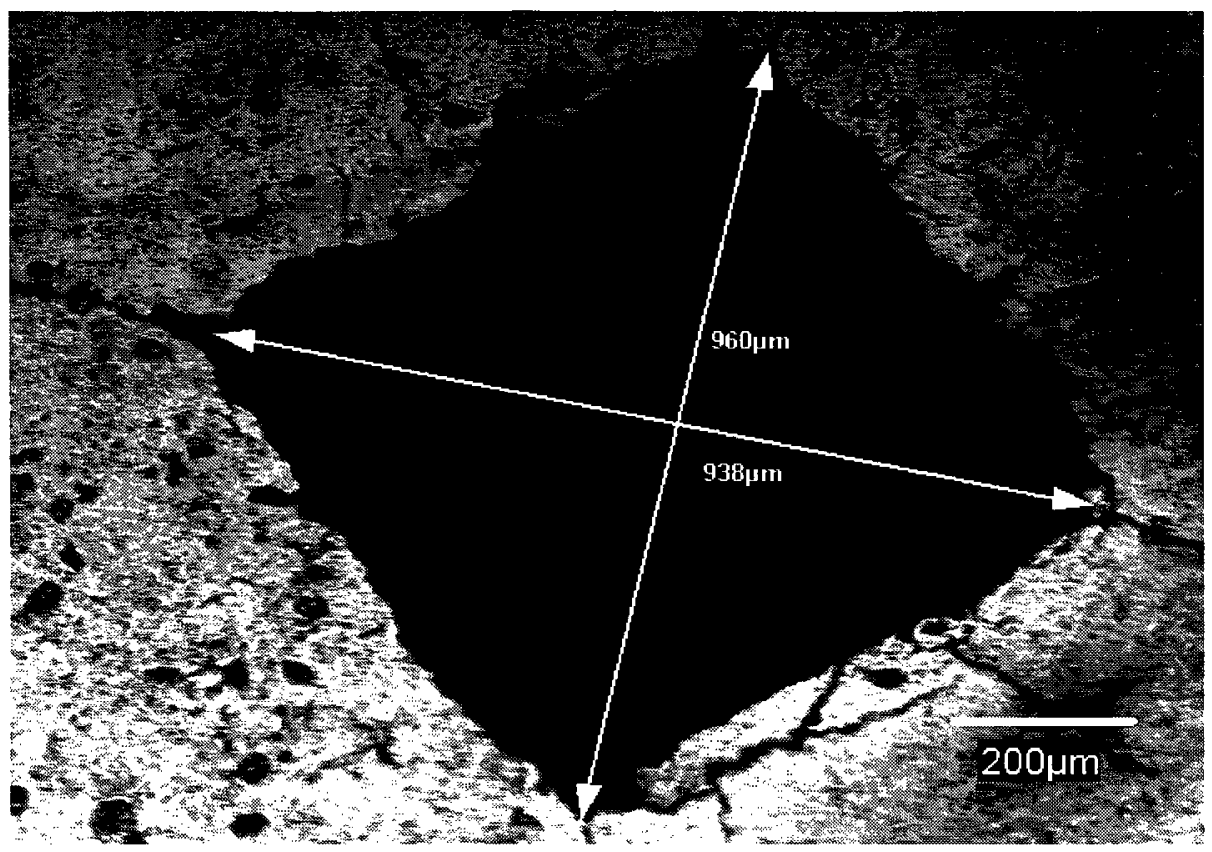

(j) Indentation mark diagonals at $P=720 \mathrm{~N}$.

Figure 4-2. Optical images of the indented $\mathrm{WC} / 10 \mathrm{Co} / 4 \mathrm{Cr}$ coating surfaces with the radial crack length and indentation mark diagonal measurements.

For the following analysis in Chapter 5, pairs of aligned well-pronounced radial cracks were identified with optical microscopy and then their crack lengths (measured from the center of the indentation mark) were added up and divided by 2 to obtain an average radial crack length $c$ for each indentation load $P$. These average radial crack lengths $c$ at the corresponding indentation loads $P$ are summarized in Table 4-1. 
Table 4-1. Average radial crack lengths $c(\mu \mathrm{m})$ at corresponding loads $P(\mathrm{~N})$.

\begin{tabular}{|c|c|}
\hline Load $P(\mathrm{~N})$ & Average radial crack length $c(\mu \mathrm{m})$ \\
\hline \multirow{5}{*}{196} & 433.0 \\
\hline & 474.5 \\
\hline & 508.5 \\
\hline & 521.5 \\
\hline & 551.0 \\
\hline \multirow{6}{*}{294} & 581.0 \\
\hline & 610.5 \\
\hline & 621.5 \\
\hline & 641.5 \\
\hline & 646.5 \\
\hline & 633.0 \\
\hline \multirow{4}{*}{392} & 653.5 \\
\hline & 678.5 \\
\hline & 684.0 \\
\hline & 730.5 \\
\hline \multirow{4}{*}{490} & 696.0 \\
\hline & 715.5 \\
\hline & 718.5 \\
\hline & 781.5 \\
\hline \multirow{5}{*}{720} & 872.5 \\
\hline & 868.5 \\
\hline & 864.0 \\
\hline & 825.5 \\
\hline & 865.0 \\
\hline
\end{tabular}


The average values of the indentation mark diagonals $l$ from three tests at each load level on the $\mathrm{WC} / 10 \mathrm{Co} / 4 \mathrm{Cr}$ coating surface were also calculated, they are summarized in Table 4-2. These values would be used in Chapter 5 for verification of the proposed fracture toughness model developed in Chapter 3.

Table 4-2. Average values of indentation diagonals $l(\mu \mathrm{m})$ in the $\mathrm{WC} / 10 \mathrm{Co} / 4 \mathrm{Cr}$ coating.

\begin{tabular}{|c|c|c|c|c|c|}
\hline Load $P(\mathrm{~N})$ & 196 & 294 & 392 & 490 & 720 \\
\hline$l(\mu \mathrm{m}),($ indentation mark \# 1) & 616.6 & 813 & 852.5 & 918 & 956.5 \\
\hline$l(\mu \mathrm{m}),($ indentation mark \#2) & 737.5 & 831.5 & 881.5 & 921.5 & 949 \\
\hline$l(\mu \mathrm{m}),($ indentation mark \#3) & 628.5 & 831 & 877 & 916.5 & 959.5 \\
\hline
\end{tabular}

\subsubsection{Substrate Hardness Measurement}

Vickers hardness of the substrate material (1080 low carbon steel) was measured in this experimental work, since this information would be used in Chapter 5 for verification of the proposed fracture toughness model developed in Chapter 3. To accomplish this, six indentation tests were performed on the 1080 low carbon steel at a load of $4.9 \mathrm{~N}$ with a Vickers hardness testing machine. The substrate hardness was evaluated using Eq. (3-5) based on the values of the indentation load $P$ and the average diagonal lengths $l$ of the indentation marks. The results are summarized in Table 4-3. 
Table 4-3. Average diagonals $l(\mathrm{~mm})$ of indentation marks on the 1080 low carbon steel substrate and the resulting hardness values $H_{s}(\mathrm{GPa})$.

\begin{tabular}{|c|c|c|}
\hline$P(\mathrm{~N})$ & $l(\mathrm{~mm})$ & $H_{s}(\mathrm{GPa})$ \\
\hline \multirow{4}{*}{4.9} & 0.0673 & 1.08 \\
\cline { 2 - 3 } & 0.0648 & 1.17 \\
\cline { 2 - 3 } & 0.0702 & 0.99 \\
\cline { 2 - 3 } & 0.0671 & 1.09 \\
\cline { 2 - 3 } & 0.0698 & 1.01 \\
\cline { 2 - 3 } & 0.0707 & 0.98 \\
\hline
\end{tabular}

Based on the testing results summarized in Table 4-3, an average Vickers hardness of the 1080 low carbon steel substrate was determined as $1.05 \mathrm{GPa}$. The data provided by UltraHard Materials Limited [63] showed that the Vickers hardness values (in $\mathrm{GPa}$ ) for the low carbon steels ranged from 0.8 to $1.8 \mathrm{GPa}$. Hence, the measured average Vickers hardness value in the present test was found acceptable for the following calculations.

\subsubsection{Coating Hardness Measurement}

Vickers hardness of the coating material ( $\mathrm{WC} / 10 \mathrm{Co} / 4 \mathrm{Cr}$ ) was also measured in this research, because this data would be required in Chapter 5 for verification of the proposed fracture toughness model developed in Chapter 3. To obtain this data, a LECO LM247AT Microhardness Tester was used. The coating surface was subjected to a $300 \mathrm{gf}$ load. To ensure non-interference of the residual fields generated by the Vickers indenter, ten indentation marks were generated at least 2.5 times Vickers diagonals away from each other and the specimen edges; the angle between the indenter and a specimen 
surface was within $2^{\circ}$ of a perpendicular [62]. The hardness values per each indentation mark are summarized in Table $4-4$. The average hardness values of the $\mathrm{WC} / 10 \mathrm{Co} / 4 \mathrm{Cr}$ coating was calculated as $1062 \mathrm{HV}$, corresponding to $1062 \mathrm{HV} \times 0.009807=10.4 \mathrm{GPa}$. The 0.009807 value is a conversion constant.

Table 4-4. A summary of hardness values $H_{c}(\mathrm{HV})$ of the $\mathrm{WC} / 10 \mathrm{Co} / 4 \mathrm{Cr}$ coating.

\begin{tabular}{|c|c|}
\hline $\begin{array}{c}\text { Indentation } \\
\text { mark \# }\end{array}$ & $H_{c}(\mathrm{HV})$ \\
\hline 1 & 1450 \\
\hline 2 & 986 \\
\hline 3 & 1180 \\
\hline 4 & 885 \\
\hline 5 & 1260 \\
\hline 6 & 714 \\
\hline 7 & 1310 \\
\hline 8 & 1010 \\
\hline 9 & 939 \\
\hline 10 & 885 \\
\hline
\end{tabular}

\subsection{Crack Profile Examination}

\subsubsection{Focused Ion Beam Technique}

To determine the in-depth radial/median crack profile and also to investigate whether the radial/median crack penetrated into the substrate under the indentation loads or not, the coated specimen was sectioned with Micrion 2500 Focused Ion Beam (FIB) microscope system.

The FIB systems use a finely focused beam of gallium ions that can be operated at either low beam current for imaging or high beam currents for high precision 
sputtering and milling (Figure 4-3). The positively charged focused ions of gallium $(\mathrm{Ga}+)$ hit the target surface and sputter a small amount of the material (Figure 4-4). Sputtering generates positive (i+) and negative (i-) ions of the target material together with some neutral atoms (n0) and secondary electrons (e-). As the ion beam scans the targeted surface, the sputtered ions and secondary electrons are collected to form an image [66].

The optional features of the Micrion system are a low energy flood gun that can be used to provide charge neutralization and a "flooding" gun that could be used to "flood" a specimen with a variety of gases. The former is utilized for non-conductive specimens that can be flooded with electrons and imaged with positive secondary ions using a positive primary ion beam. The latter is used to provide selective gas assisted etching through interaction of the primary gallium beam with the secondary gases. A "flooding" gun could also be used for the selective deposition of either conductive or insulating materials by decomposition of the deposition gas by the primary ion beam.

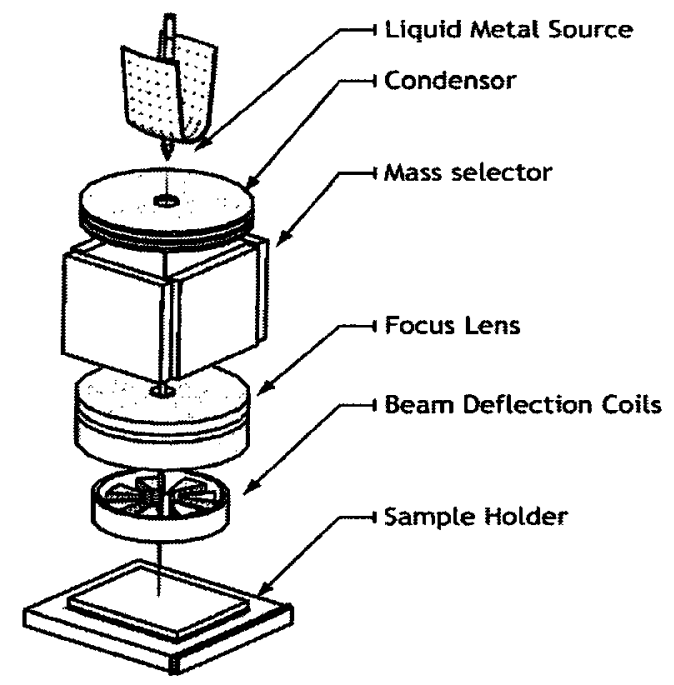

Figure 4-3. Focused Ion Beam system set-up [64]. 


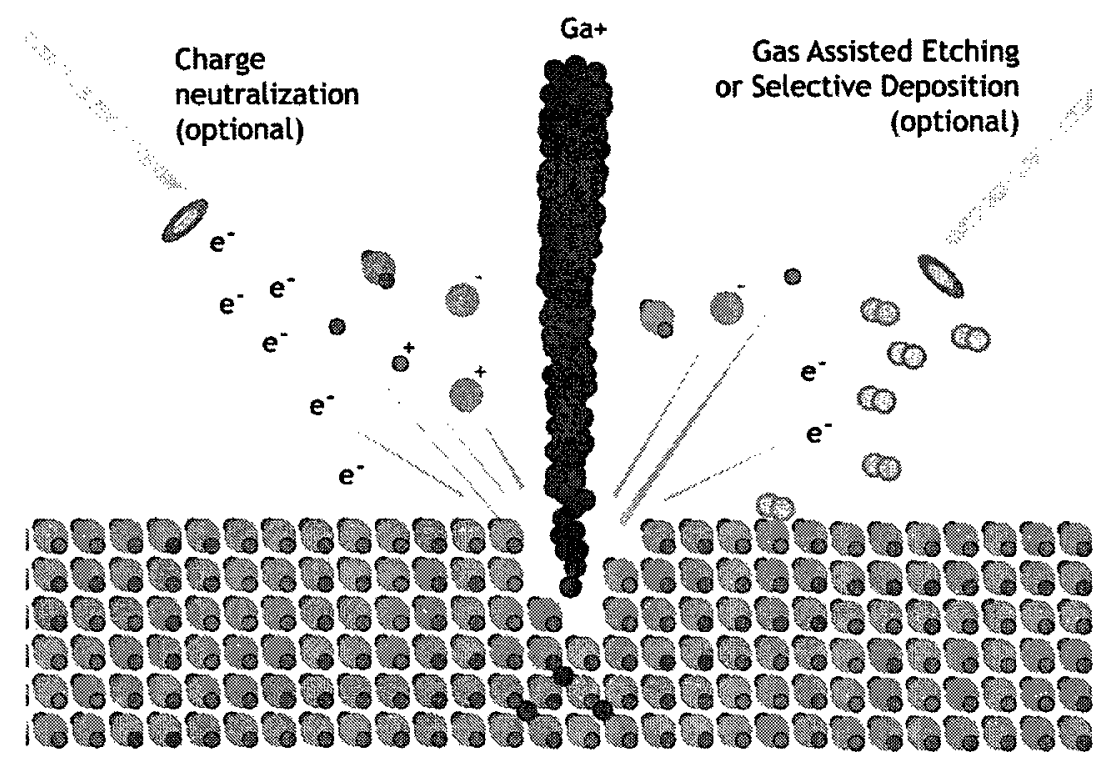

Figure 4-4. Focused Ion Beam system sputtering process [64].

\subsubsection{Examination Procedures}

Out of all indentation marks generated at different loads, five were selected for the destructive examination. Each mark corresponded to a specific load such as: $P=196 \mathrm{~N}, P=294 \mathrm{~N}, P=392 \mathrm{~N}, P=490 \mathrm{~N}$ and $P=720 \mathrm{~N}$ respectively. As clearly observed in Figure 4-5, radial cracks emanate from the opposite corners of each indentation mark. Thus, a couple of radial cracks per load were selected and sectioned at two locations along their length as shown in Figure 4-5. 


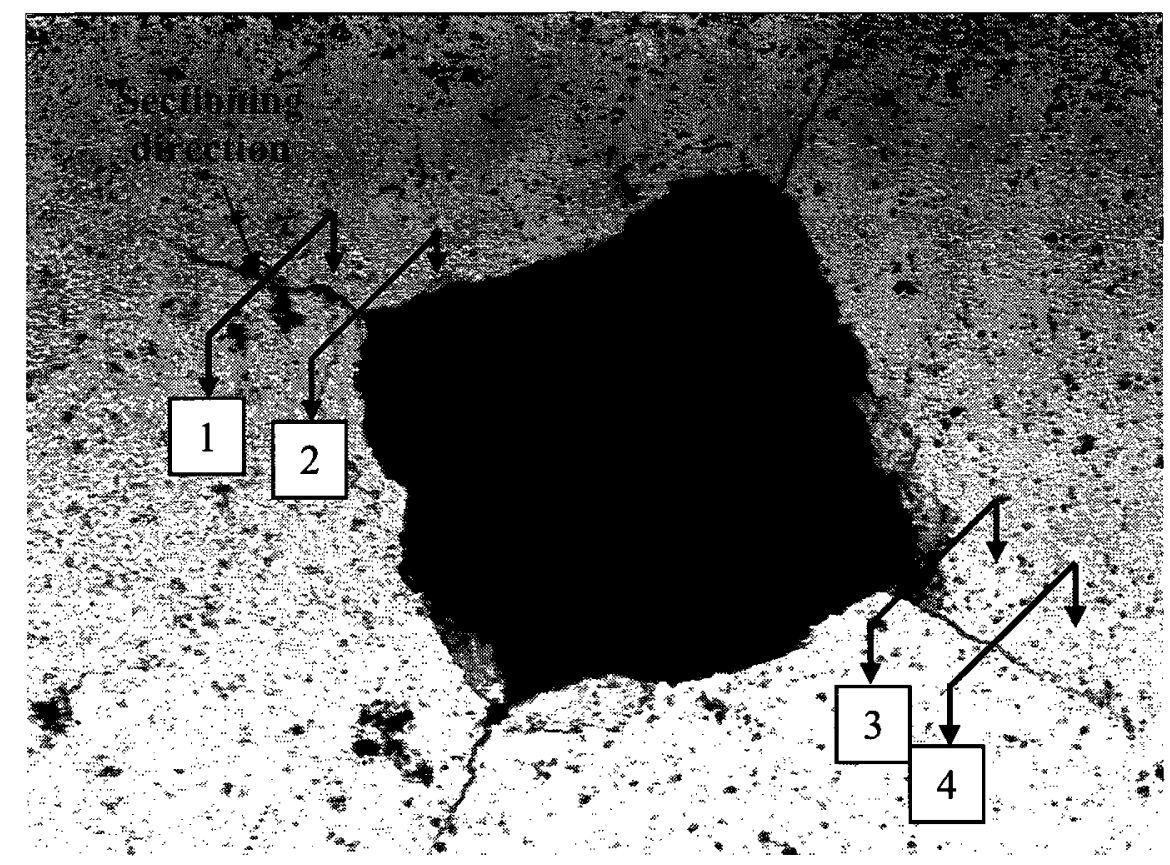

Figure 4-5. Typical radial/median cracks sectioned at four locations along their lengths in the plane normal to the coating surface.

\subsubsection{Post-Indentation Coating Surface Examination}

The indented surfaces were examined from the top using FIB technique at all indentation loads. The radial and edge cracks, as shown in Figure 4-6, were observed in all images. 


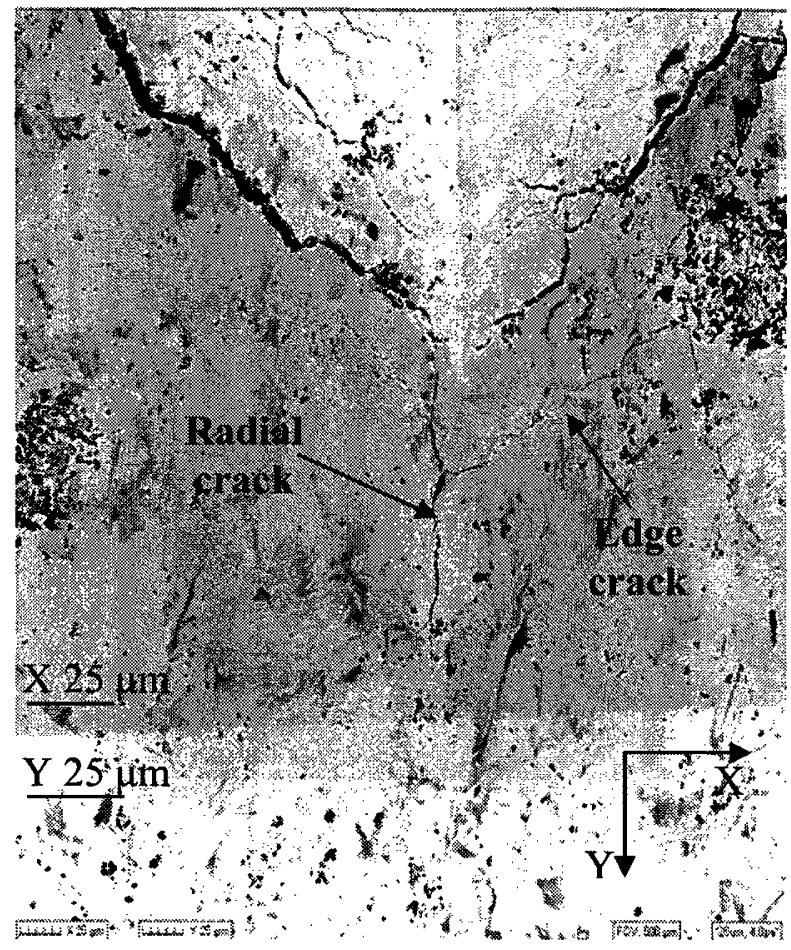

(a) under $P=196 \mathrm{~N}$

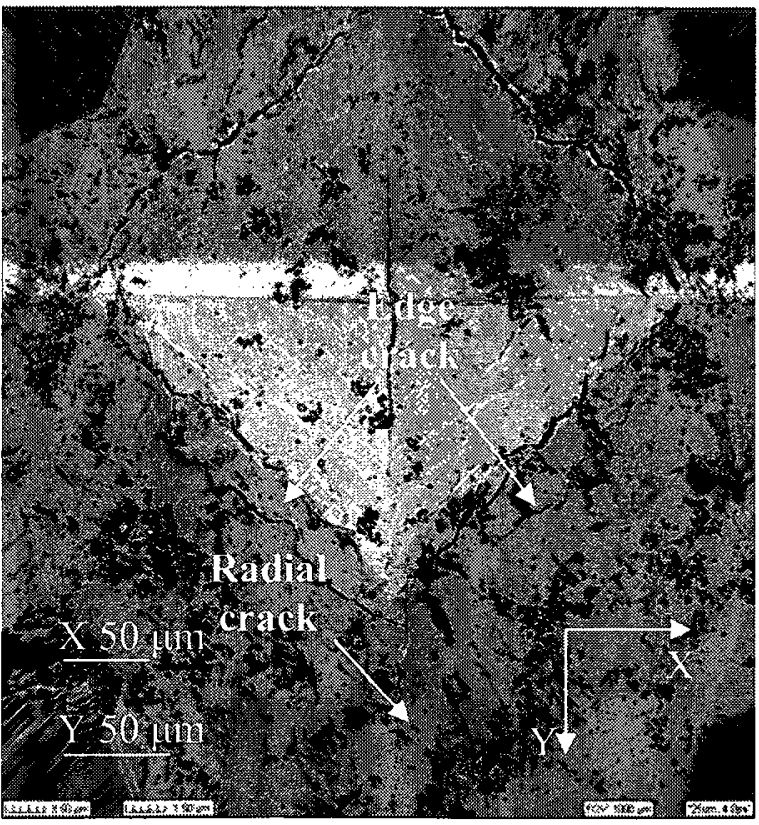

(c) under $P=392 \mathrm{~N}$

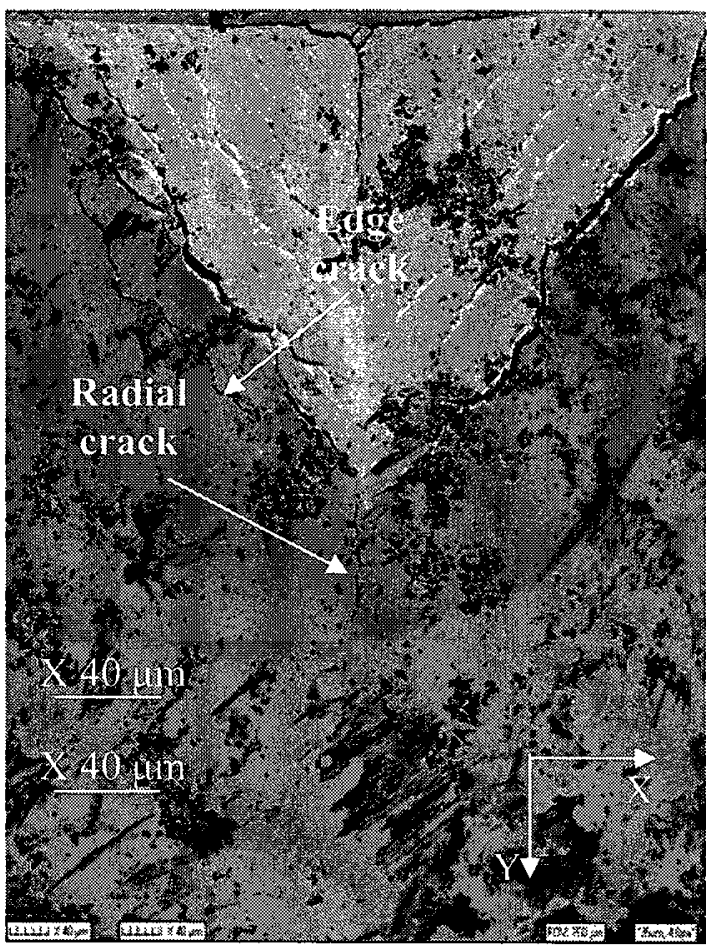

(b) under $P=294 \mathrm{~N}$

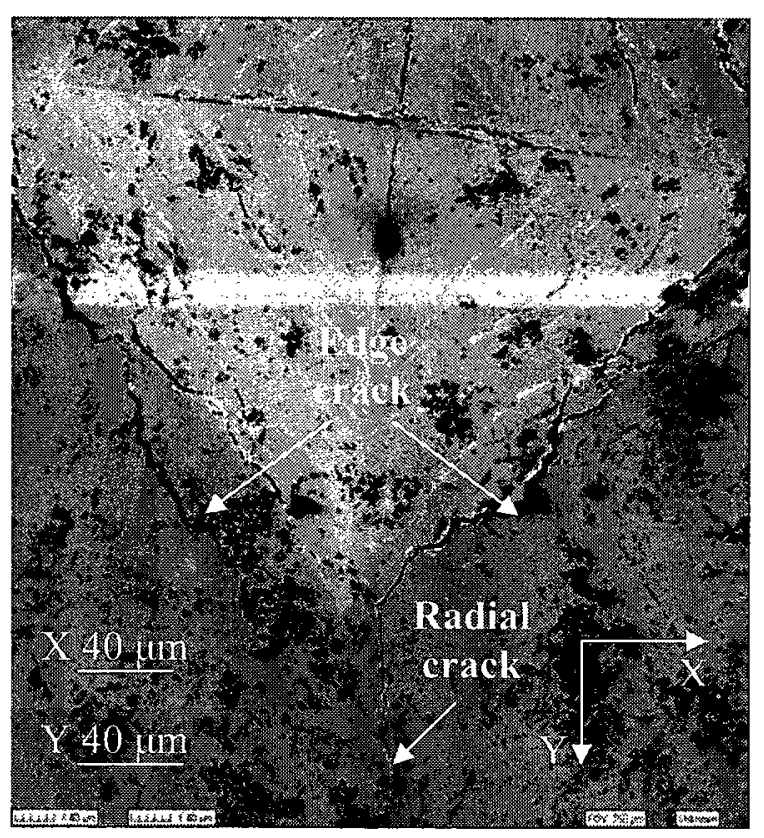

(d) under $P=490 \mathrm{~N}$ 


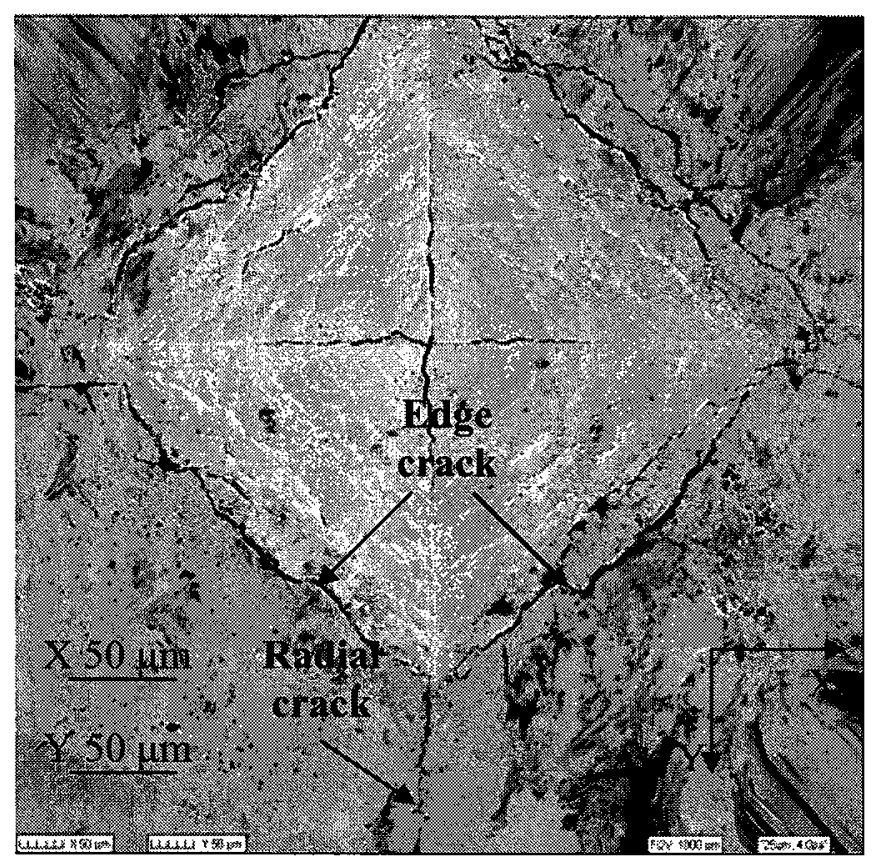

(e) under $P=720 \mathrm{~N}$

Figure 4-6. Top views of the indented $\mathrm{WC} / 10 \mathrm{Co} / 4 \mathrm{Cr}$ coating surface at all indentation loads.

It has been observed that when the $\mathrm{WC} / 10 \mathrm{Co} / 4 \mathrm{Cr}$ coating surfaces were indented with an increasing normal load, it typically resulted in a coating fracture along the indentation edges and cracks emanating from the corners of the indentation marks, i.e., radial cracks. For example, at $P=196 \mathrm{~N}$, which was the lowest among applied indentation loads, the edge cracks developed along the indentation edges; the radial cracks also originated from the corners of the indentation marks, as shown in Figure 4-6 (a). At higher indentation loads (from $P=294 \mathrm{~N}$ to $P=720 \mathrm{~N}$ ), a similar fracture pattern was observed, as shown in Figure 4-6 (b)-(e), although the edge cracks gradually became more pronounced along the indentation edges and eventually coalesced, as shown in Figure 4-6 (e). 


\subsubsection{Crack Profile Images}

The FIB technique was used to obtain the images of the crack profiles in the coating/substrate cross-sections. In all the images at either lower or higher loads, four types of cracks were observed. The median and lateral cracks were observed in the coating/substrate cross-sections; and the radial and edge cracks were observed on the coating surfaces. The correlation between observed cracks is demonstrated in Figure 4-7. The summarized results of the cross-section examinations are presented in Figure 4-8 to Figure 4-12. Each image represents one cut along the radial crack (see Figure 4-5) at its corresponding indentation load. 


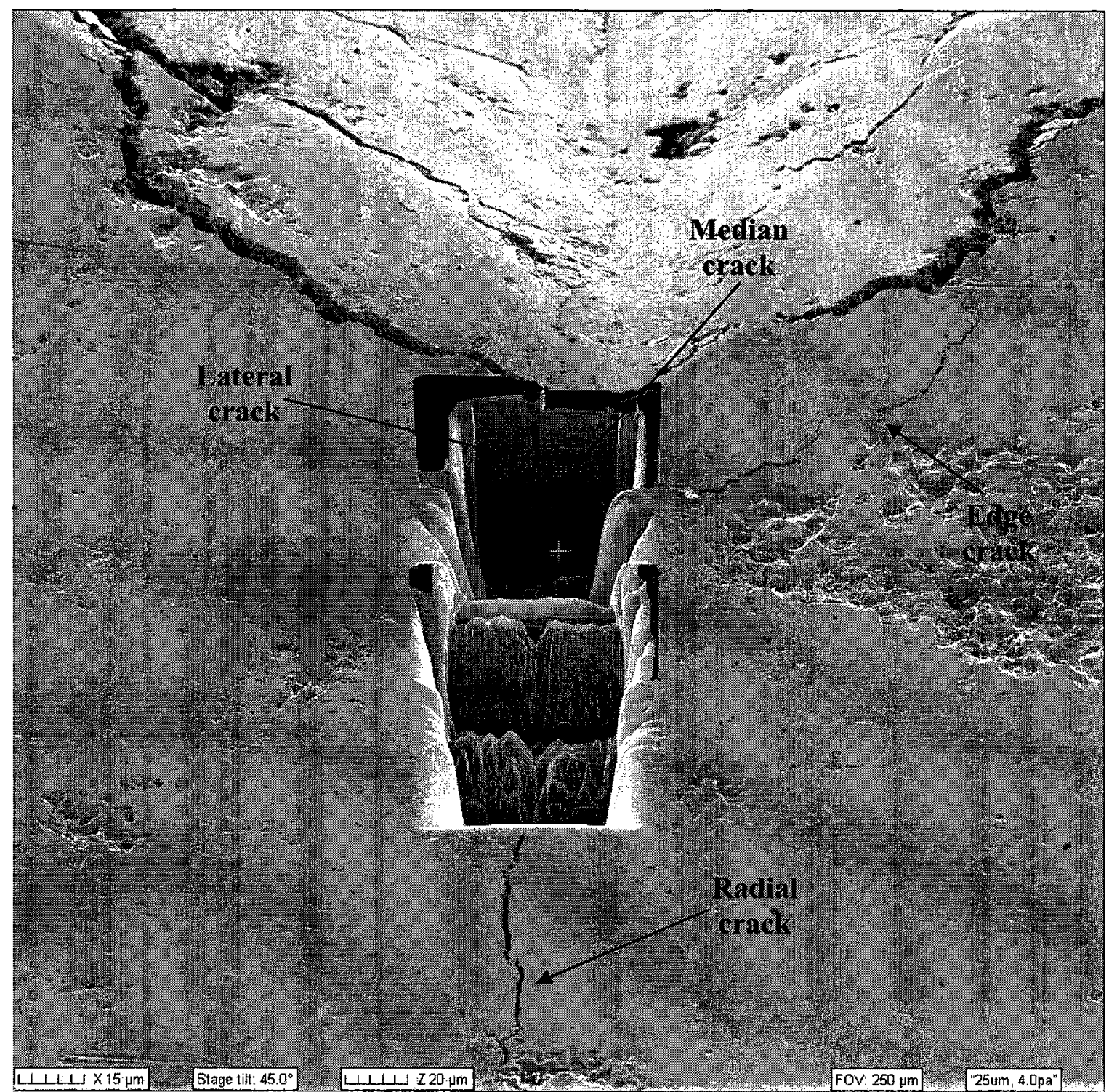

Figure 4-7. Correlation between radial, median, lateral and edge cracks. 


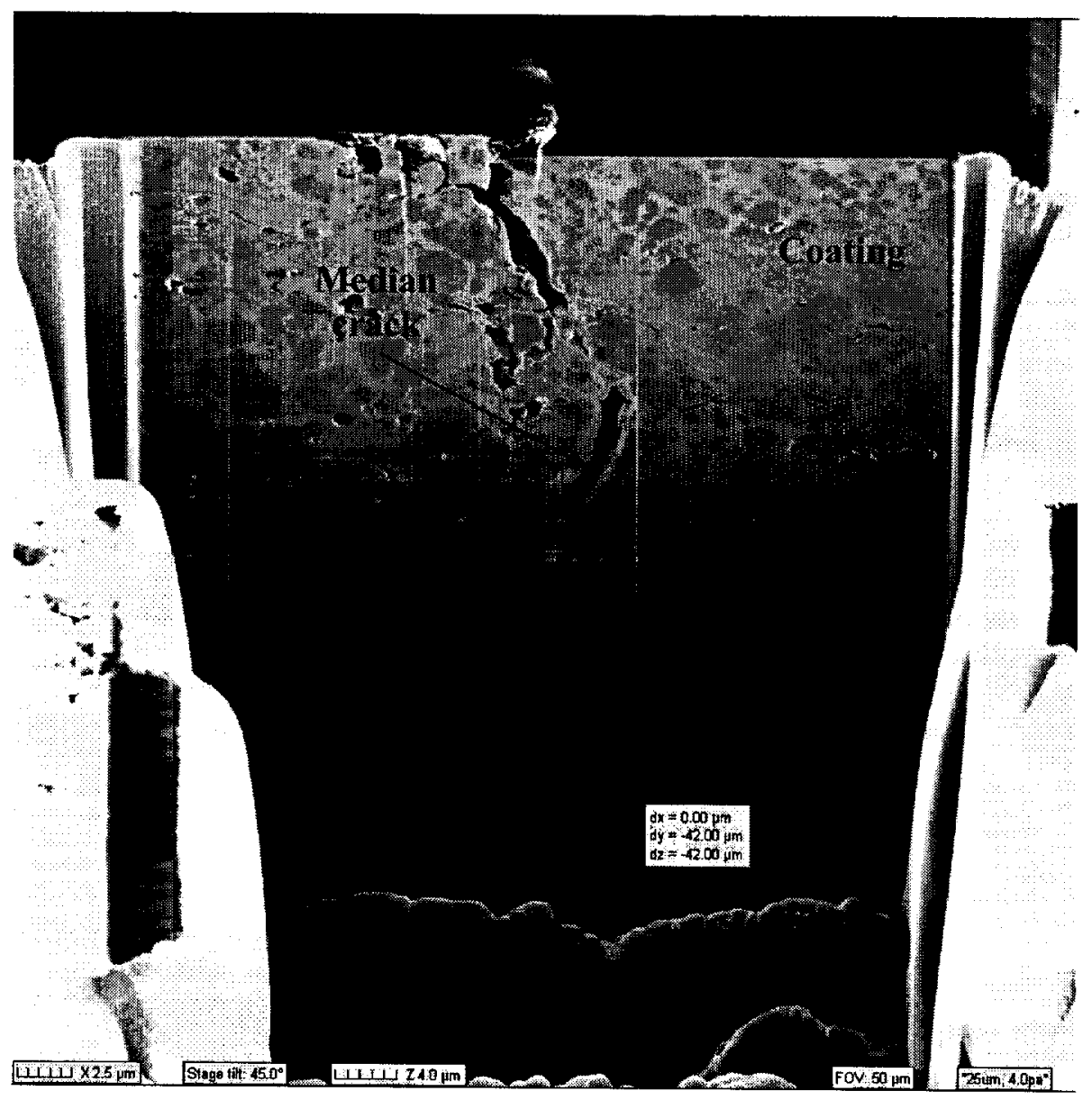

(a) Section 1 . 


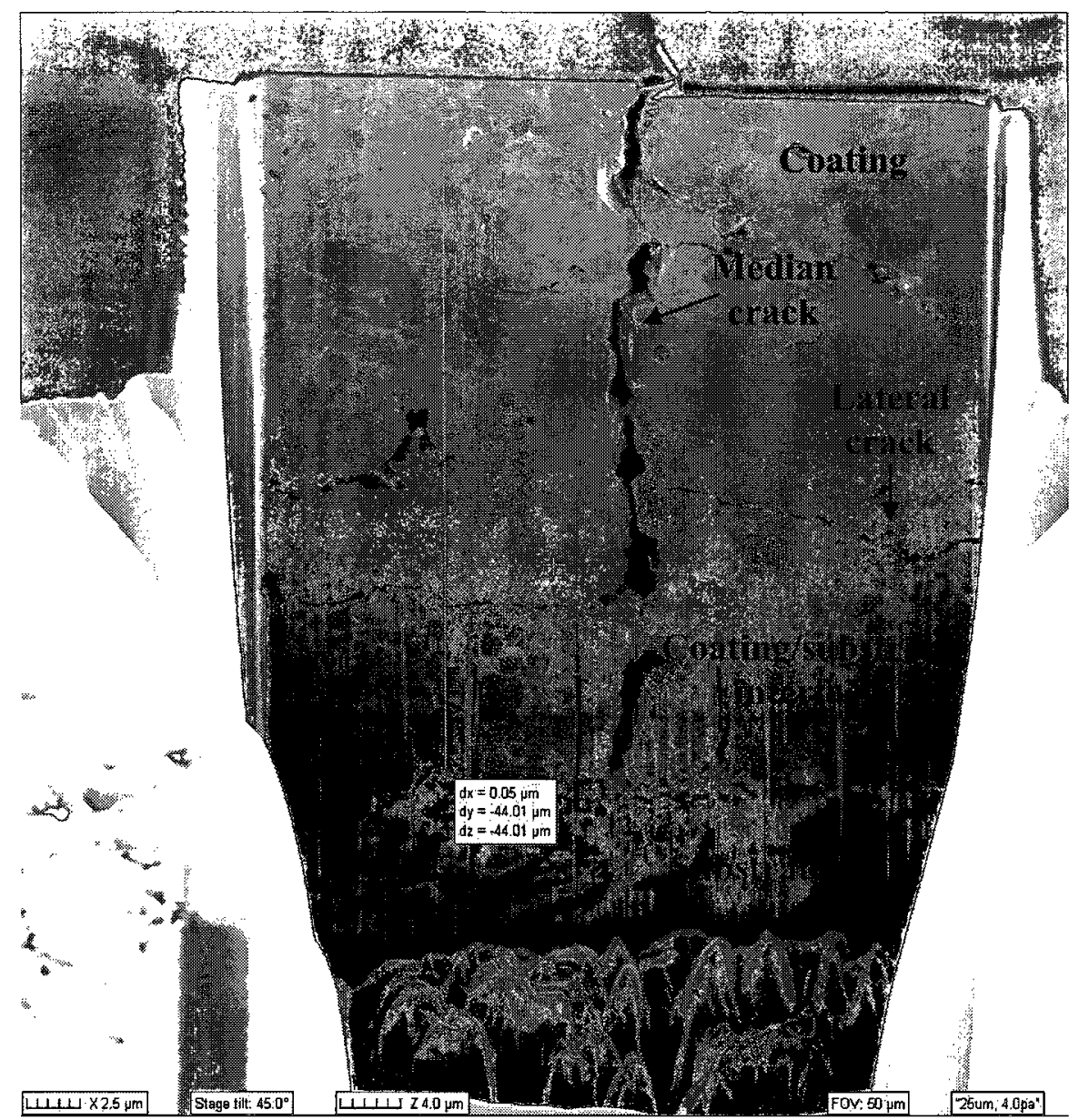

(b) Section 2. 


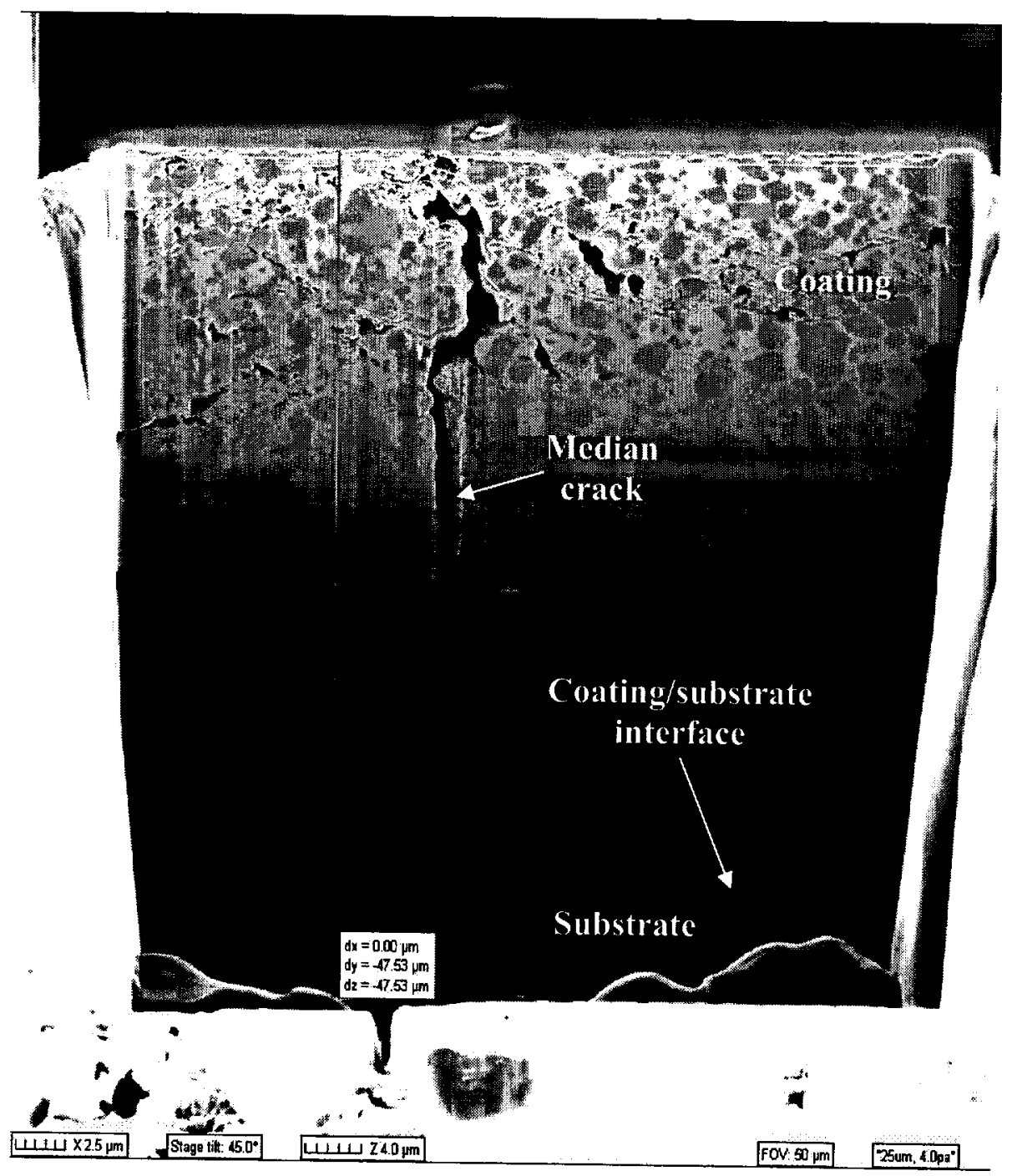

(c) Section 3 . 


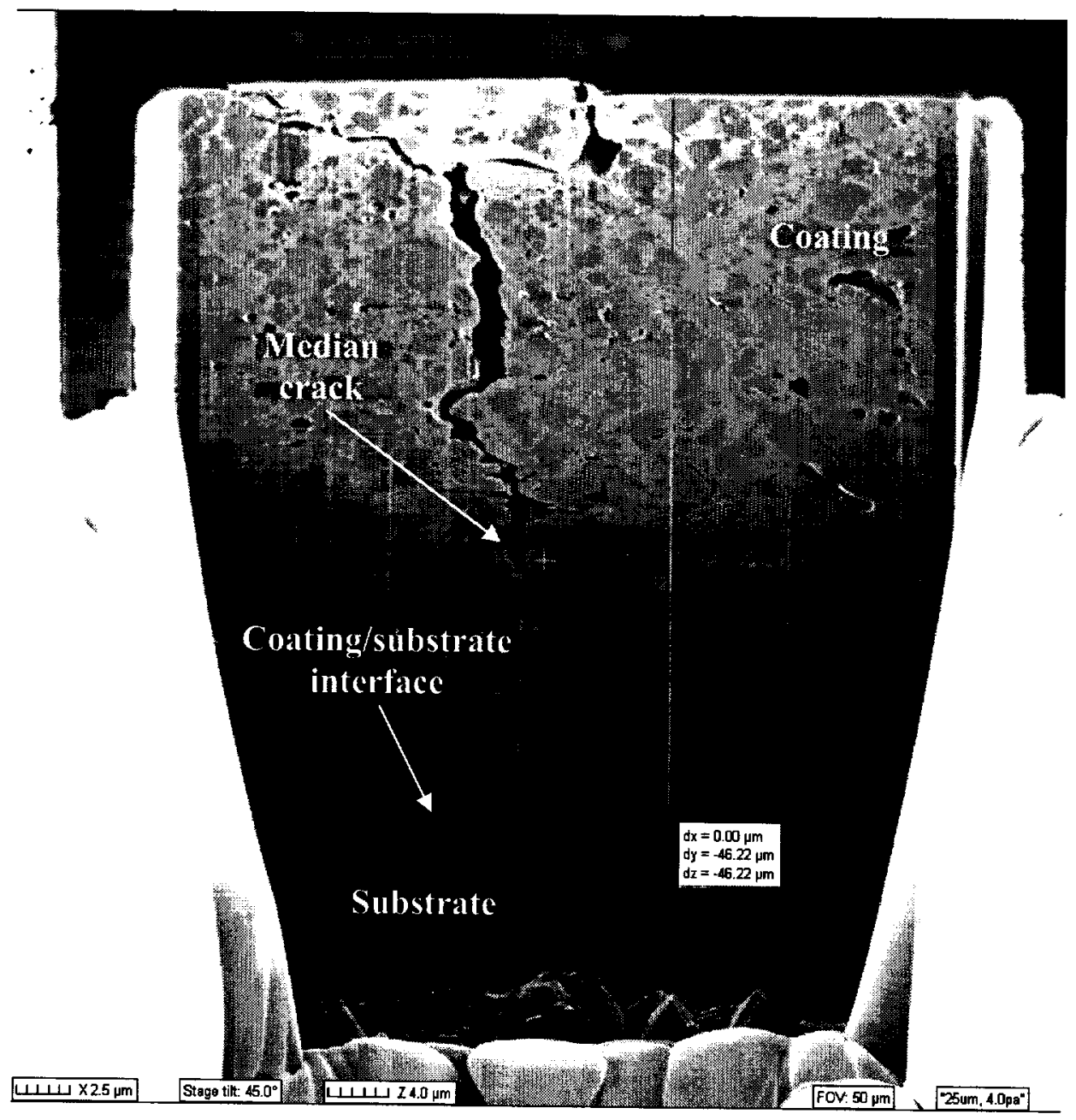

(d) Section 4 .

Figure 4-8. Cross-sectional views of the $\mathrm{WC} / 10 \mathrm{Co} / 4 \mathrm{Cr}$ coating/1080 steel substrate system at $P=196 \mathrm{~N}$. 


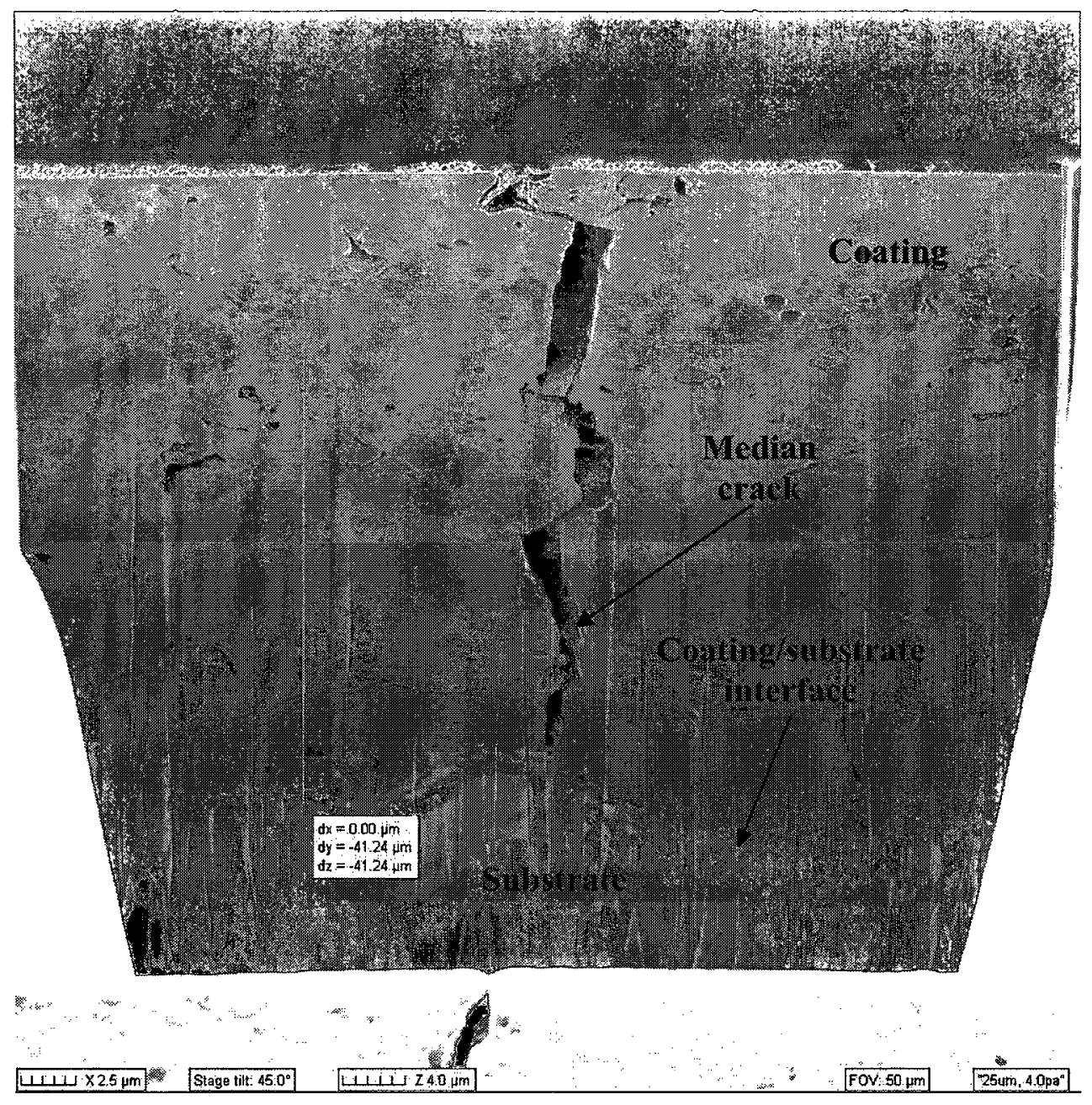

(a) Section 1. 


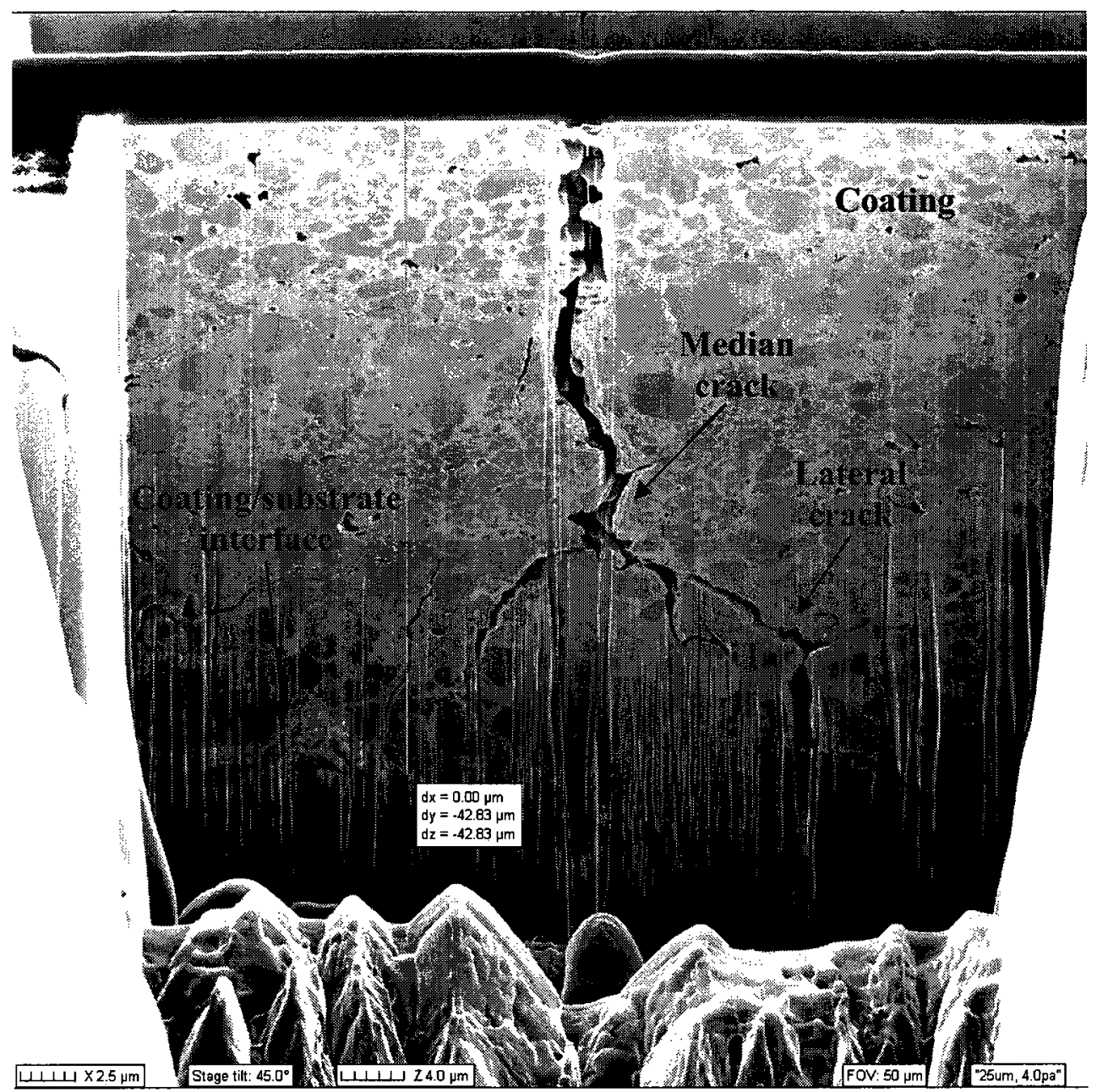

(b) Section 2. 


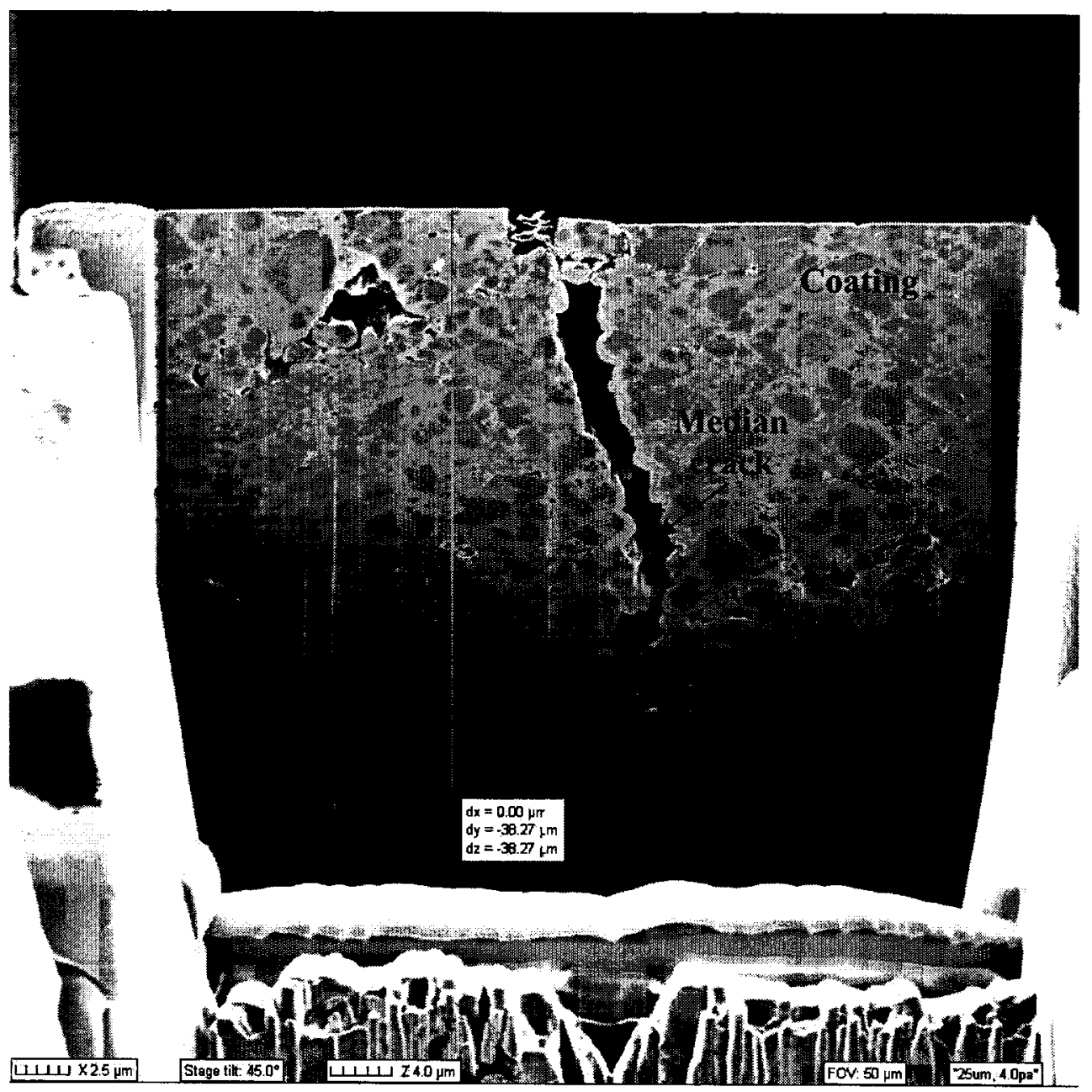

(c) Section 3 . 


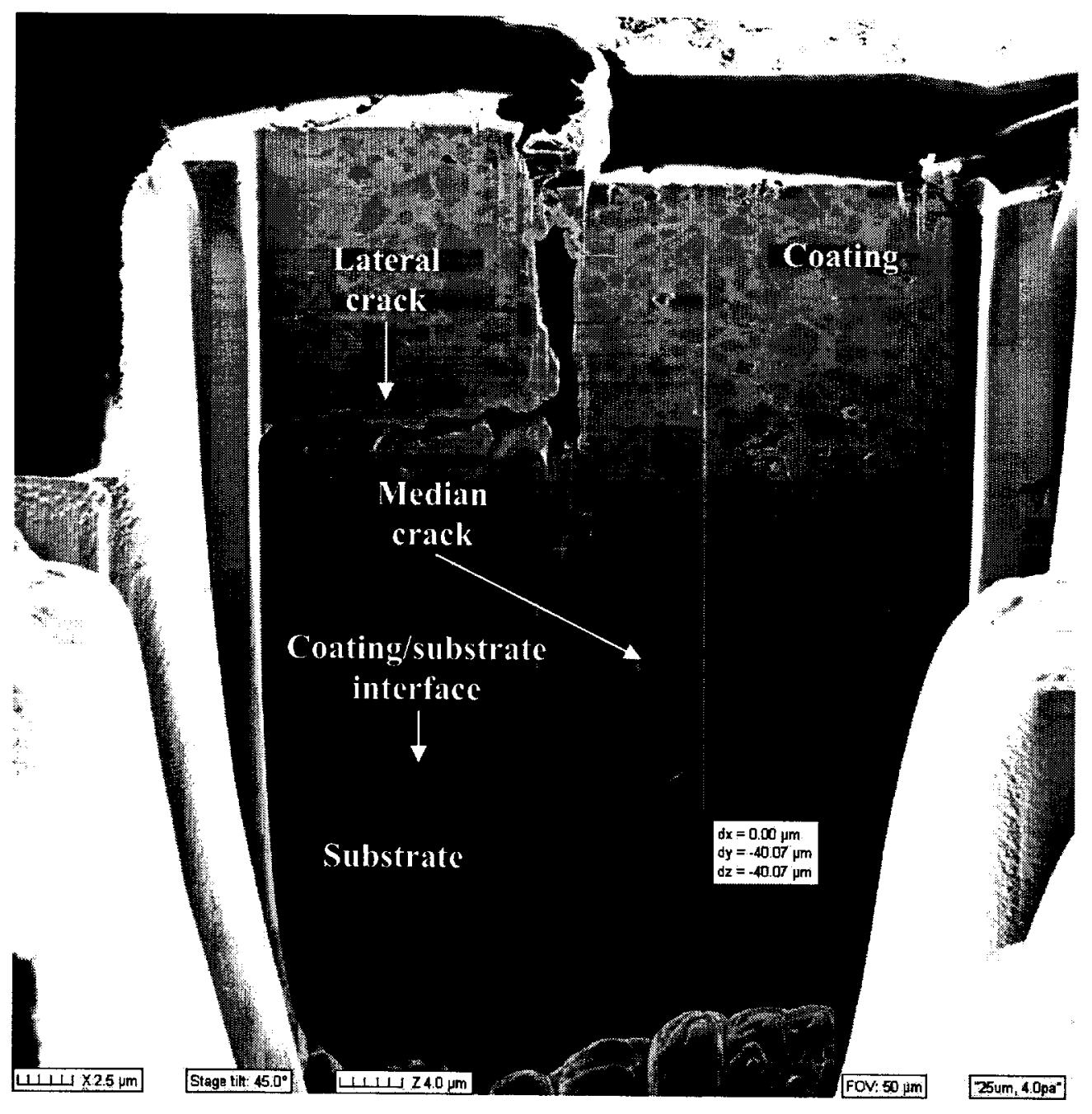

(d) Section 4.

Figure 4-9. Cross-sectional views of the $\mathrm{WC} / 10 \mathrm{Co} / 4 \mathrm{Cr}$ coating/1080 steel substrate system at $P=294 \mathrm{~N}$. 


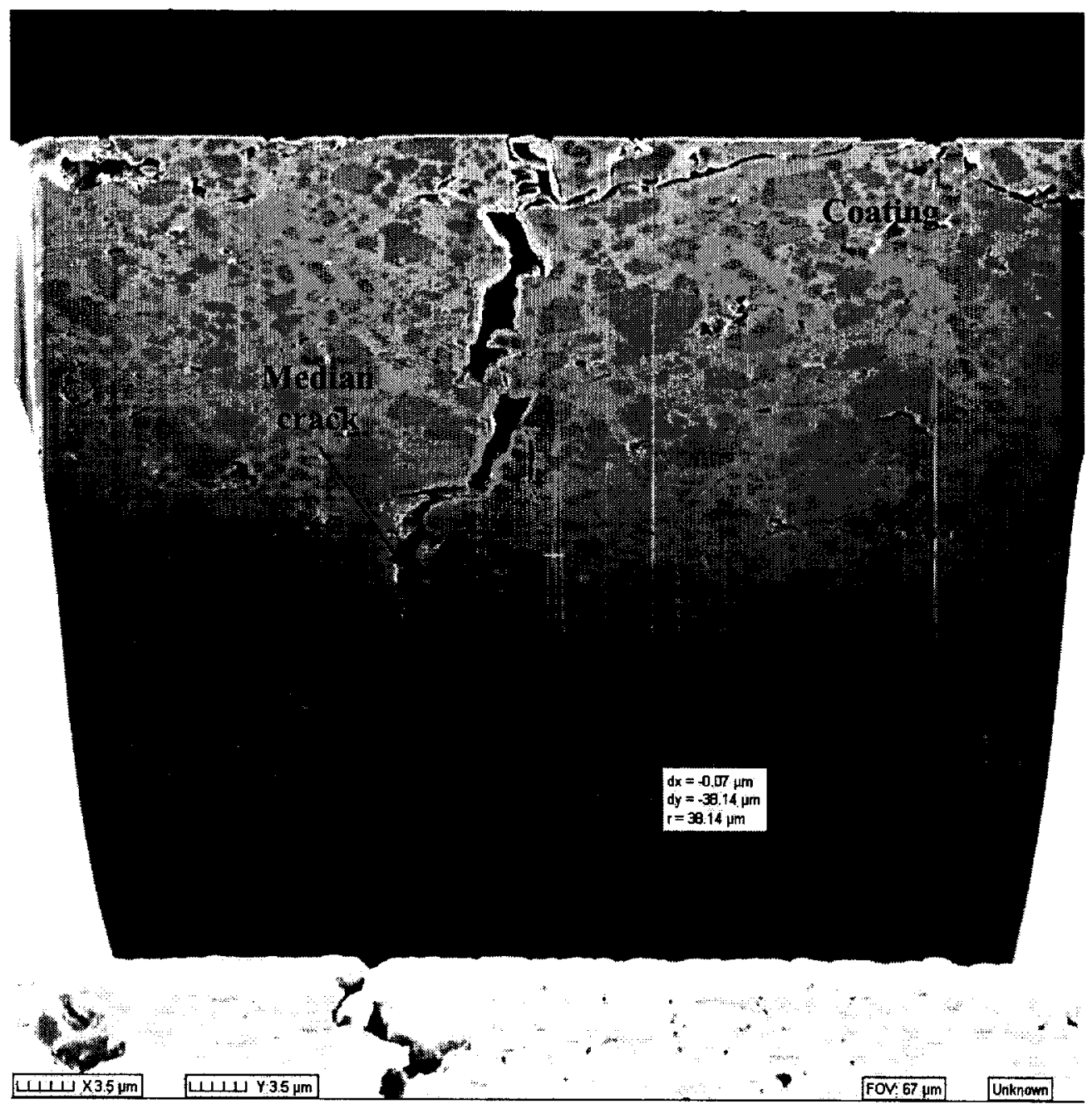

(a) Section 1 . 


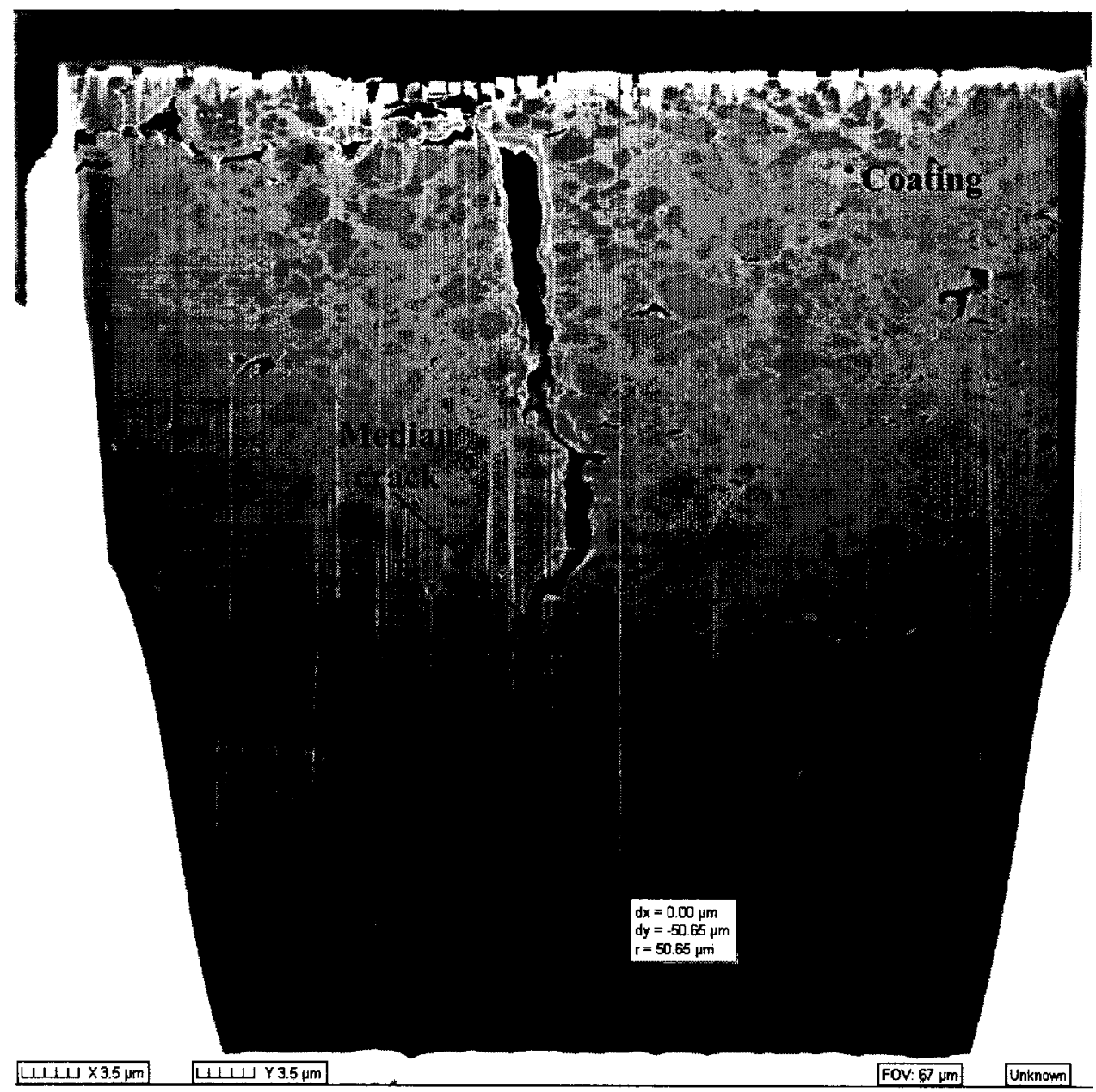

(b) Section 2. 


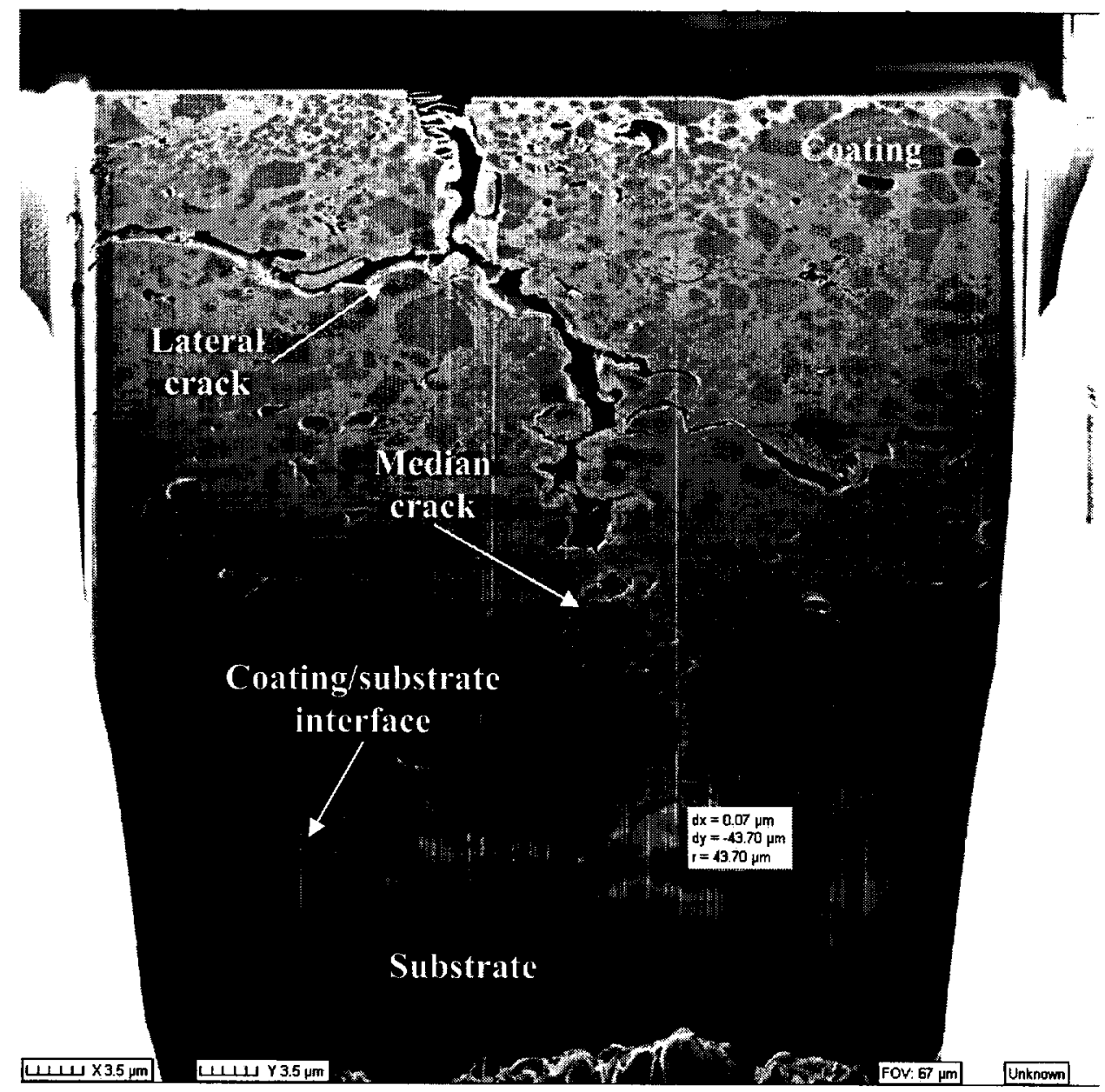

(c) Section 3 . 


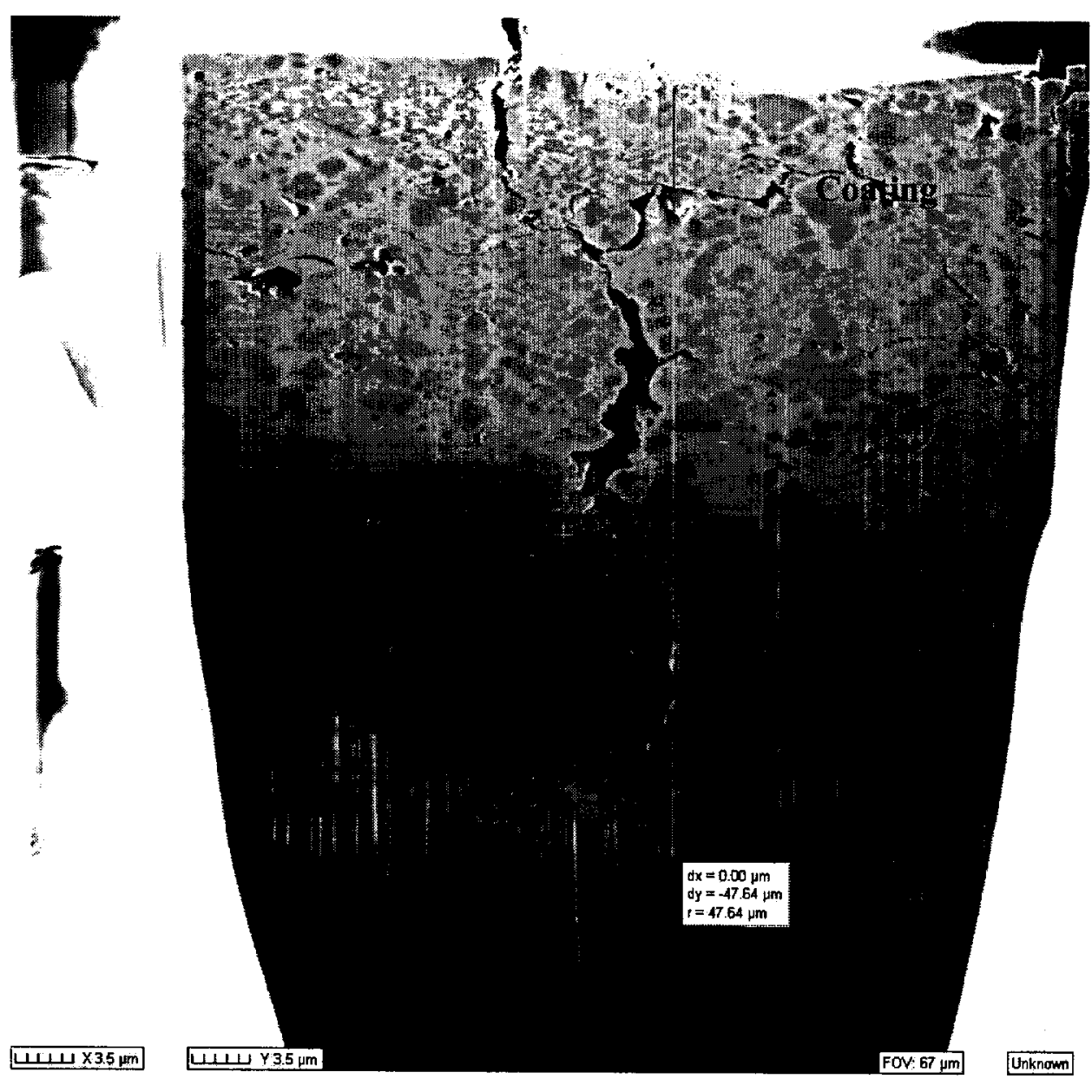

(d) Section 4.

Figure 4-10. Cross-sectional views of the $\mathrm{WC} / 10 \mathrm{Co} / 4 \mathrm{Cr}$ coating/1080 steel substrate system at $P=392 \mathrm{~N}$. 


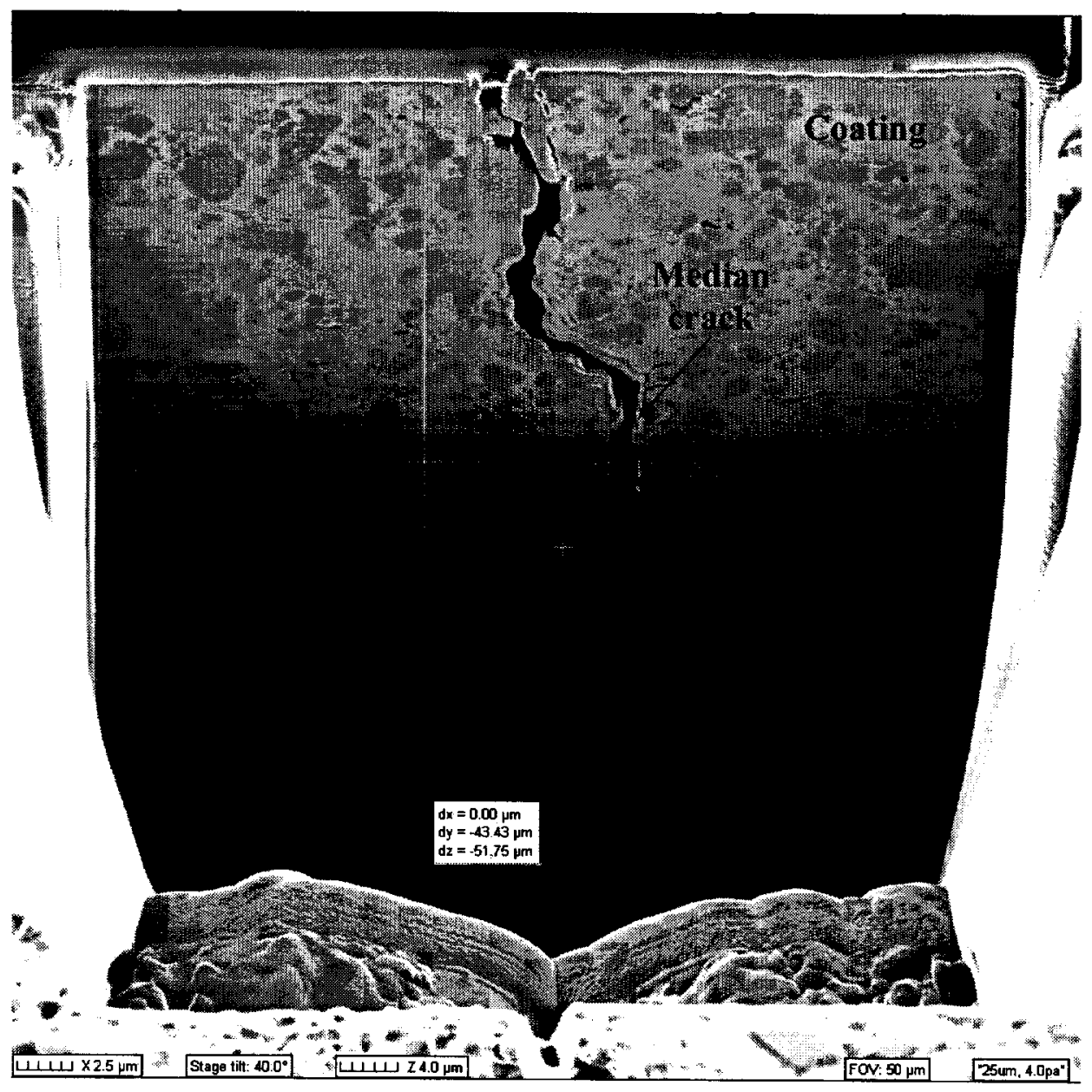

(a) Section 1 . 


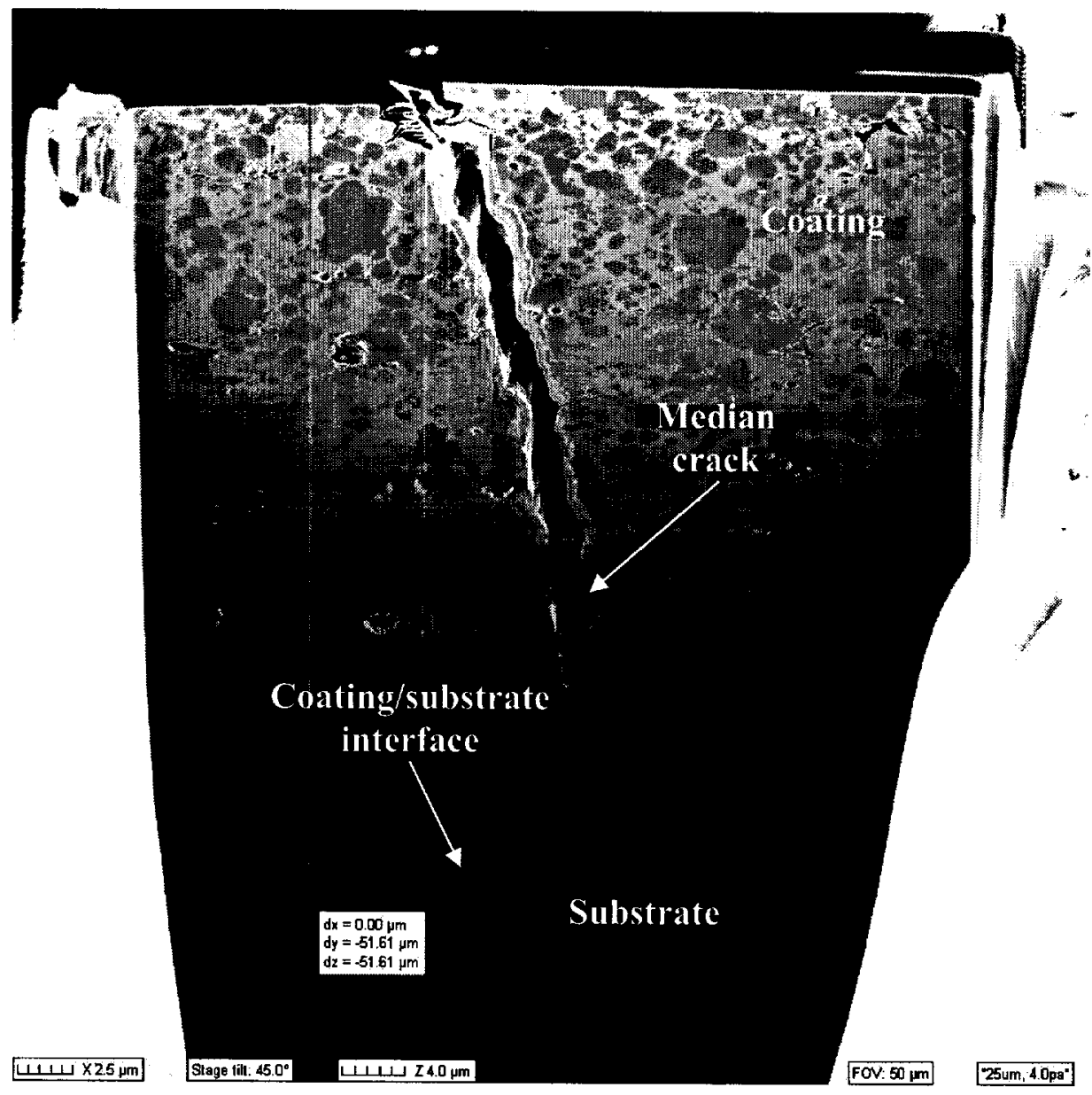

(b) Section 2 . 


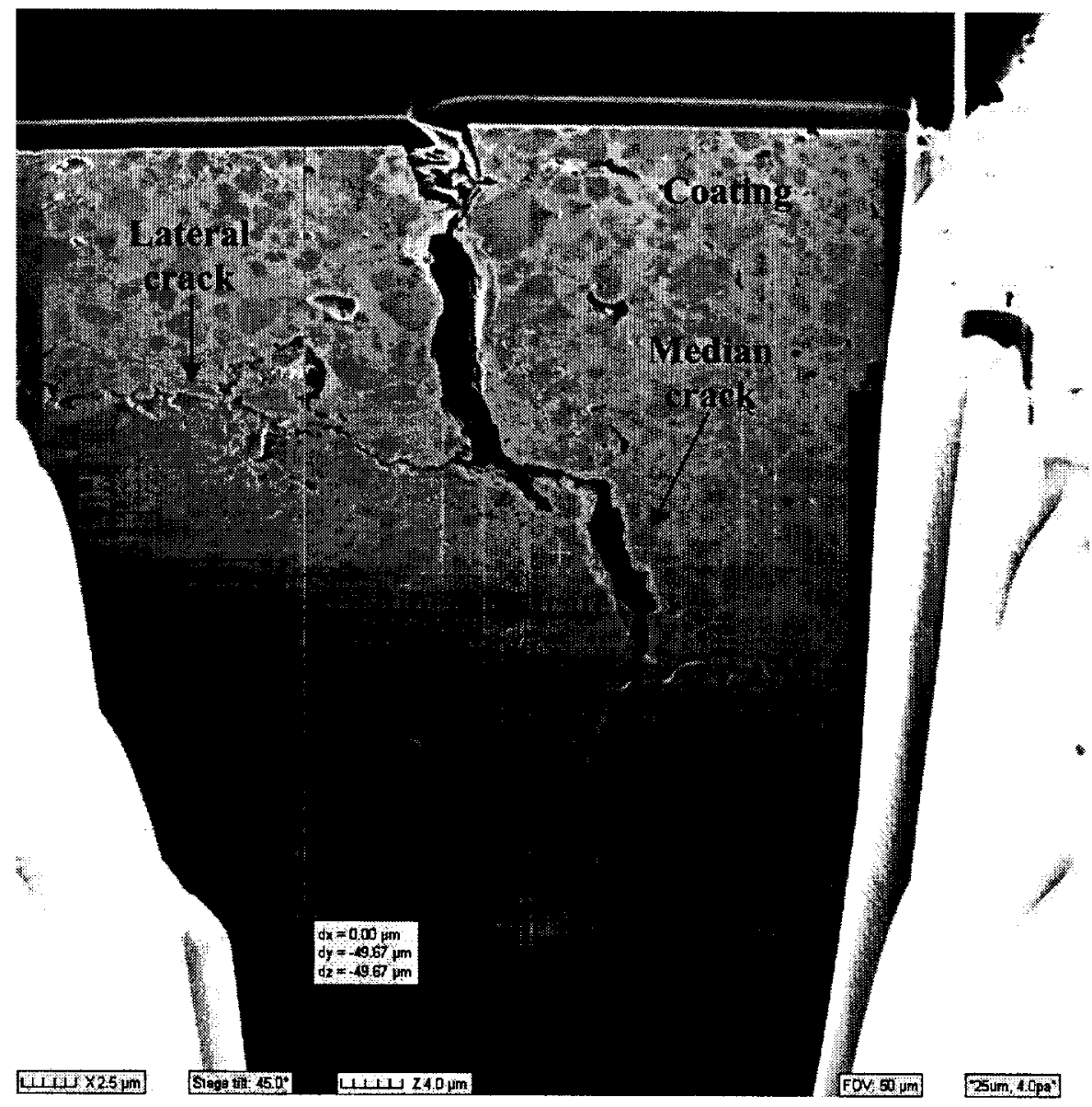

(c) Section 3. 


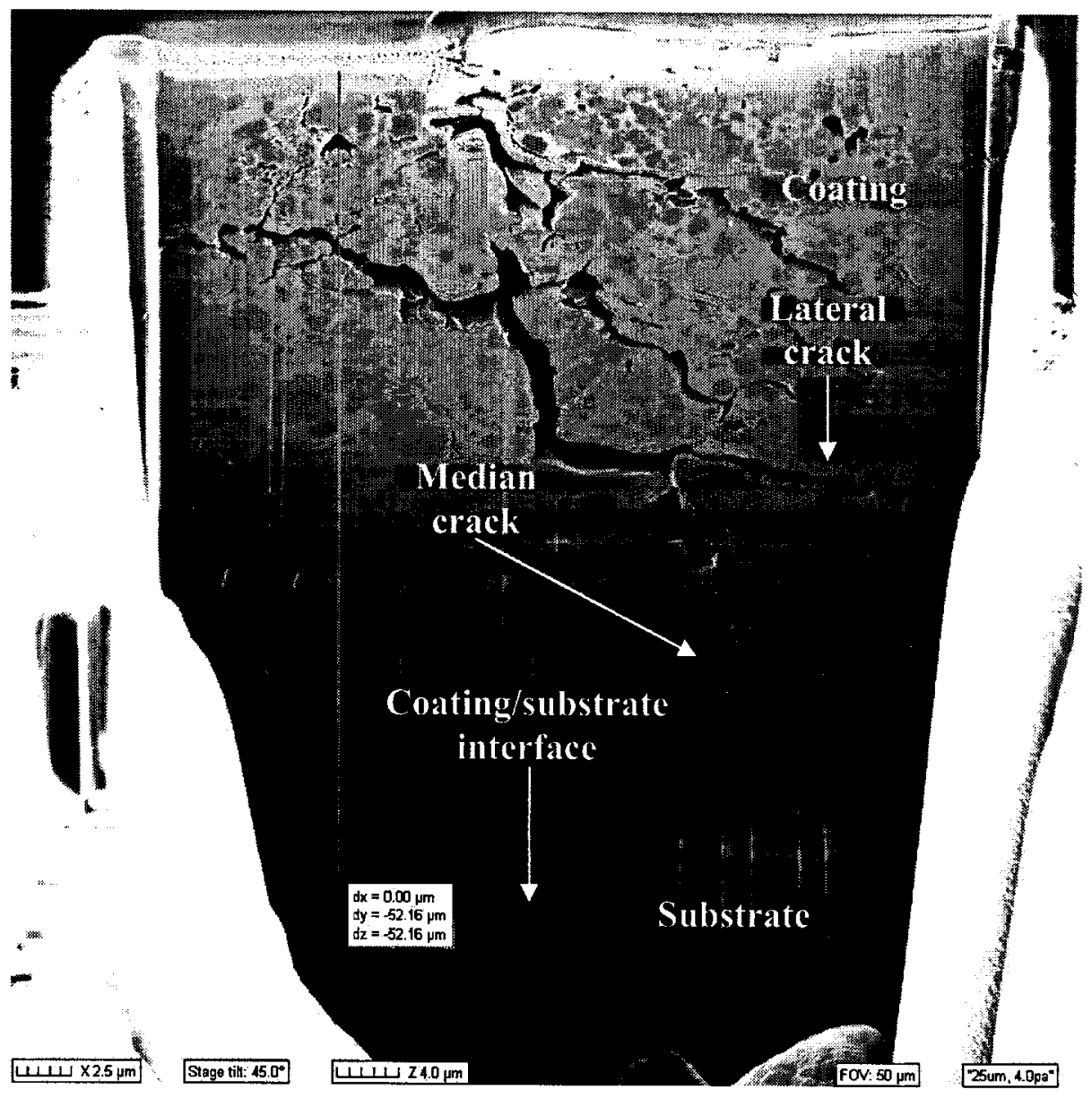

(d) Section 4 .

Figure 4-11. Cross-sectional views of the $\mathrm{WC} / 10 \mathrm{Co} / 4 \mathrm{Cr}$ coating/1080 steel substrate system at $P=490 \mathrm{~N}$. 


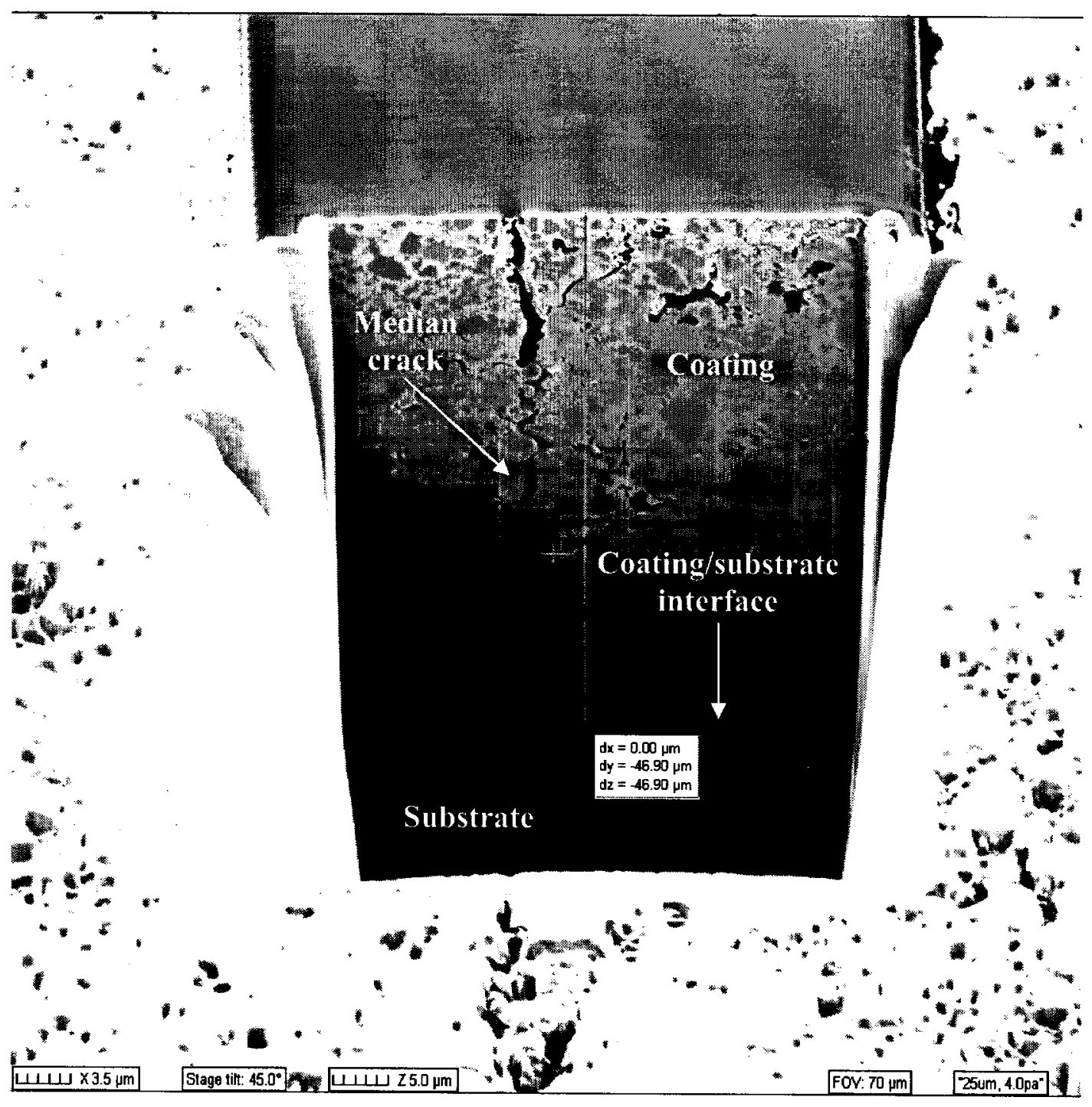

(a) Section 1 . 


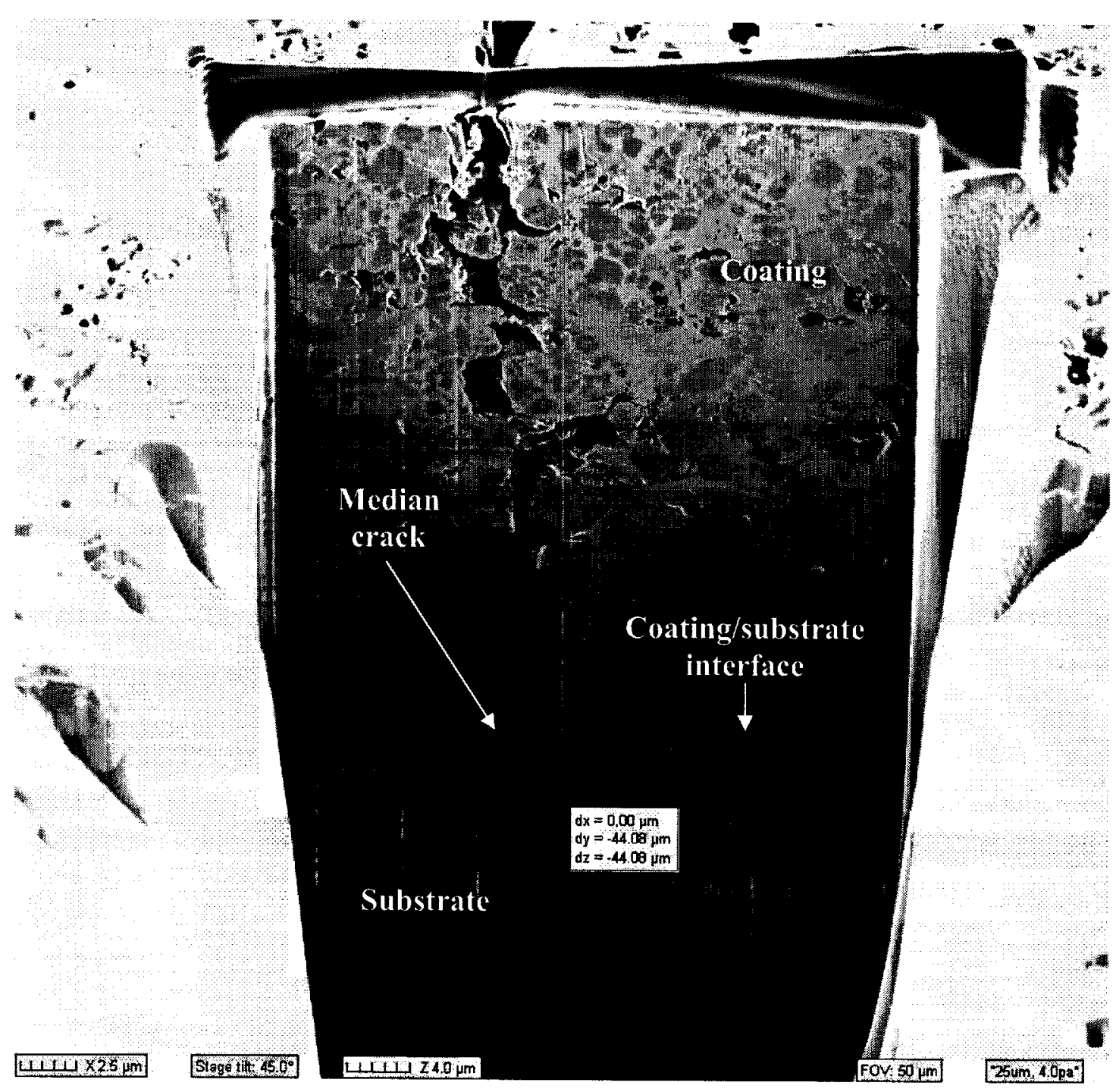

(b) Section 2 . 


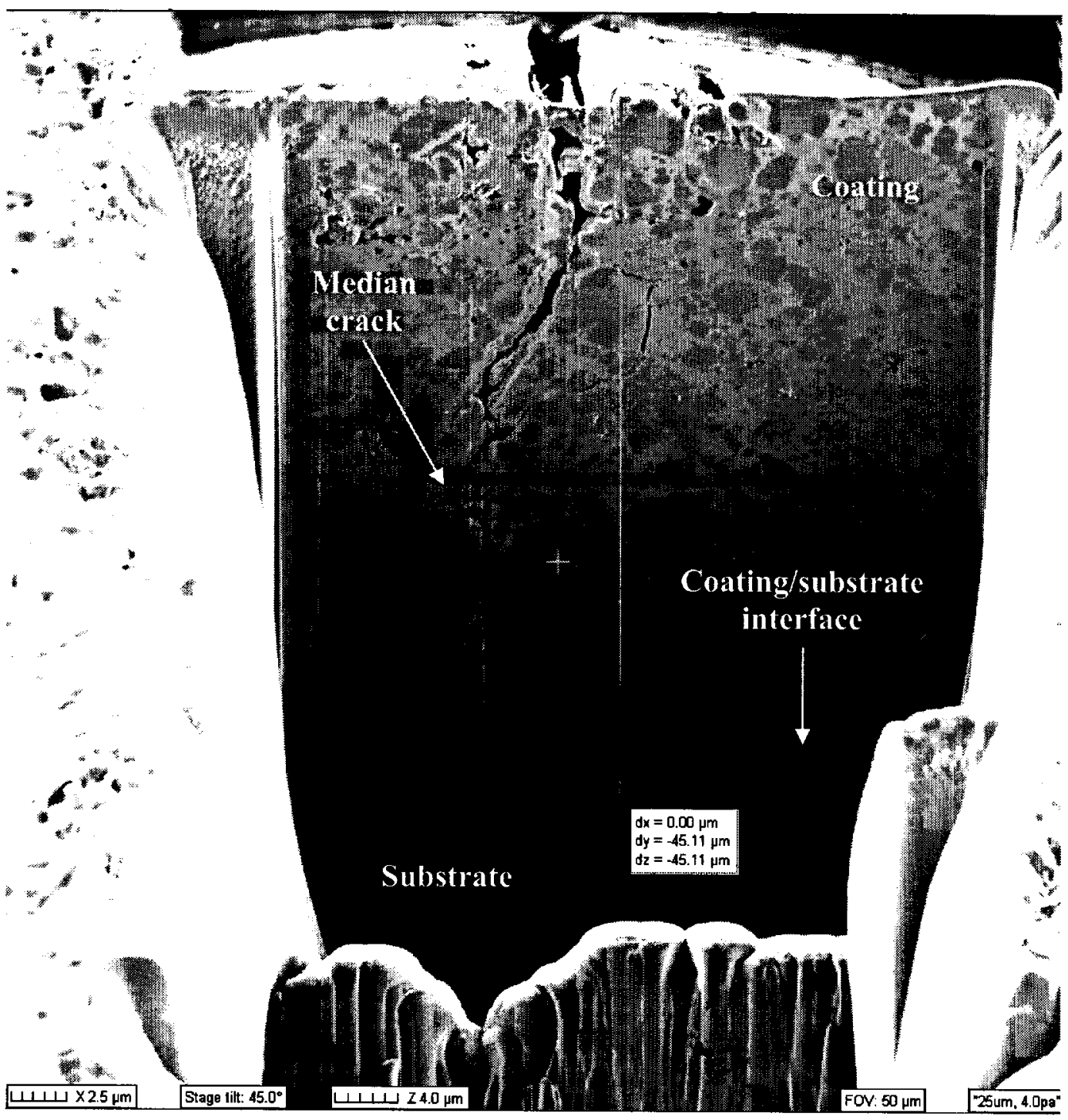

(c) Section 3. 


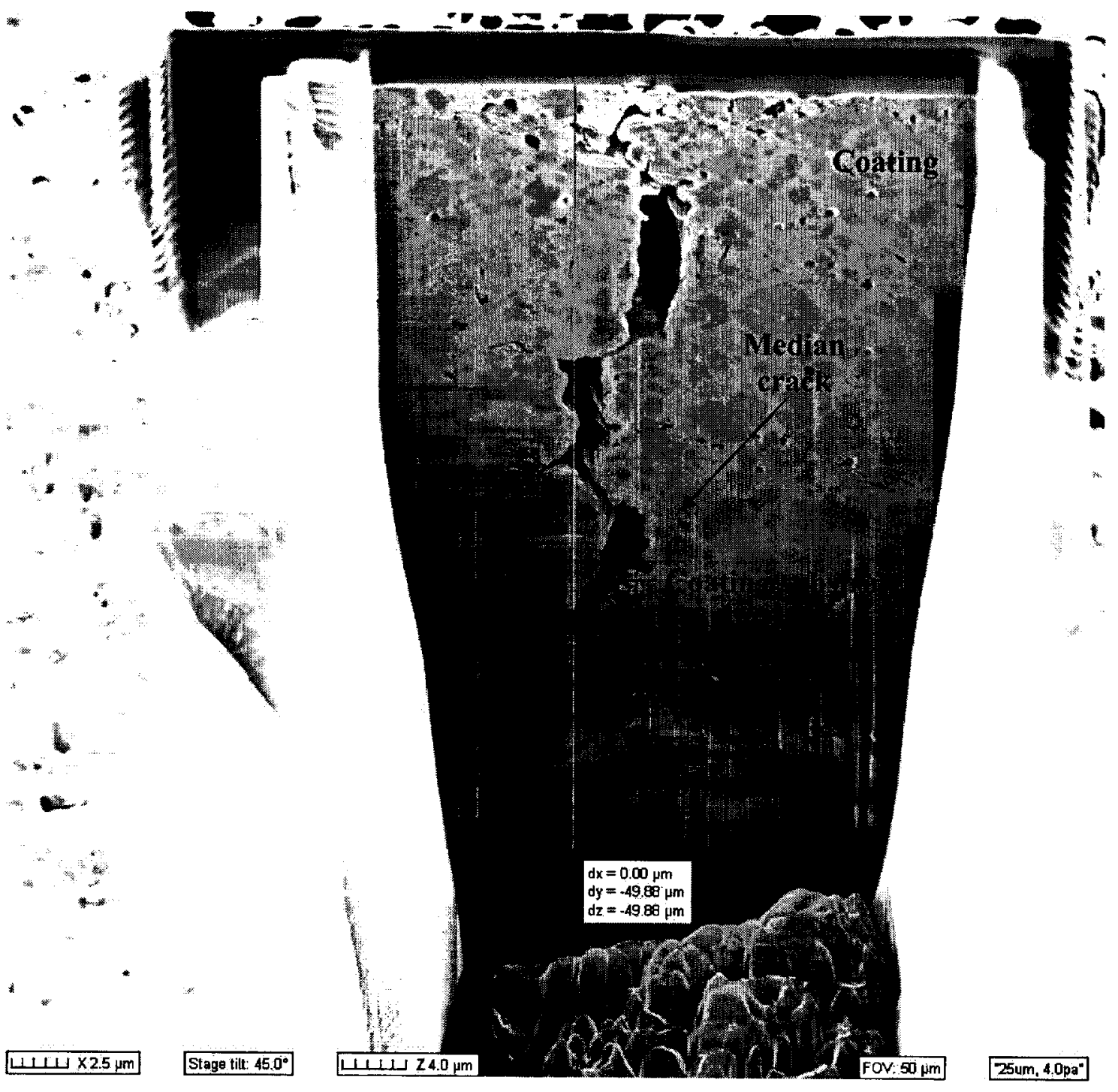

(d) Section 4 .

Figure 4-12. Cross-sectional views of the $\mathrm{WC} / 10 \mathrm{Co} / 4 \mathrm{Cr}$ coating/1080 steel substrate system at $P=720 \mathrm{~N}$. 


\subsubsection{Analysis of Crack Profiles}

As demonstrated in Figure 4-8 to Figure 4-12, the cross-section of the $\mathrm{WC} / 10 \mathrm{Co} / 4 \mathrm{Cr}$ coating/1080 low carbon steel substrate system consists of the coating layer and the substrate with an interface. The darker spots in the microstructure of the coating material (the top part of the cross-section) are tungsten-chromium ( $\mathrm{WC} / \mathrm{Cr})$ and the lighter spots in-between are cobalt $(\mathrm{Co})$ solid solution. A change of microstructure in the bottom part of the cross-section indicates a 1080 low carbon steel substrate.

It is evident that the $\mathrm{WC} / 10 \mathrm{Co} / 4 \mathrm{Cr}$ coating exhibited brittle behavior. The dominant fracture patterns observed in all the cross-sections were radial/median cracks, regardless of whether the coating was subjected to lower or higher indentation loads, as summarized in Table 4-5. The edge cracks were also observed growing along the indentation edges at all load levels, but these cracks were disregarded since our focus was only on the radial/median fracture pattern. Occasionally, a lateral crack intersecting the median crack was also observed. Its presence was sometimes a cause for the median crack arrest. However, in most cases, the coating/substrate interface was a restrictive factor; it remained well-bonded during the indentation tests whether the indentation load was lower of higher. For the purposes of this study, the most important concern is whether the fracture (crack) propagated into the coating from the top surface and then was confined within the coating, which resulted in elliptical shape radial/median crack. An assumption of radial/median crack ellipticity was made in Chapter 3 . The experimental observations have confirmed this assumption. 
Table 4-5. A summary of the observed cracks.

\begin{tabular}{|c|c|c|c|}
\hline $\begin{array}{l}\text { Load P } \\
(\mathrm{N})\end{array}$ & $\begin{array}{l}\text { Section } \\
\#\end{array}$ & $\begin{array}{c}\text { Coating thickness } \\
h_{c}(\mu \mathrm{m})\end{array}$ & Crack behavior \\
\hline \multirow{4}{*}{196} & 1 & 47.53 & $\begin{array}{l}\text { Radial/median crack arrested by interface; } \\
\text { lateral crack present }\end{array}$ \\
\hline & 2 & 46.22 & Radial/median crack arrested by interface \\
\hline & 3 & 42.00 & Radial/median crack arrested by interface \\
\hline & 4 & 44.01 & $\begin{array}{l}\text { Radial/median crack arrested by interface; } \\
\text { lateral crack present }\end{array}$ \\
\hline \multirow{4}{*}{294} & 1 & 41.24 & $\begin{array}{c}\text { Radial/median crack arrested by interface; } \\
\text { lateral crack present }\end{array}$ \\
\hline & 2 & 42.83 & $\begin{array}{c}\text { Radial/median crack arrested by } \\
\text { lateral crack }\end{array}$ \\
\hline & 3 & 38.27 & $\begin{array}{l}\text { Radial/median crack arrested by } \\
\text { lateral crack }\end{array}$ \\
\hline & 4 & 40.04 & $\begin{array}{l}\text { Radial/median crack arrested by interface; } \\
\text { lateral crack present }\end{array}$ \\
\hline \multirow{4}{*}{392} & 1 & 38.14 & Radial/median crack arrested by interface \\
\hline & 2 & 50.65 & $\begin{array}{c}\text { Radial/median crack arrested by } \\
\text { lateral crack }\end{array}$ \\
\hline & 3 & 43.7 & $\begin{array}{c}\text { Radial/median crack arrested by interface; } \\
\text { lateral crack present }\end{array}$ \\
\hline & 4 & 47.64 & $\begin{array}{l}\text { Radial/median crack arrested by interface; } \\
\text { lateral crack present }\end{array}$ \\
\hline \multirow{4}{*}{490} & 1 & 43.43 & $\begin{array}{l}\text { Radial/median crack arrested by interface; } \\
\text { lateral crack present }\end{array}$ \\
\hline & 2 & 51.61 & $\begin{array}{c}\text { Radial/median crack arrested by interface; } \\
\text { lateral crack present }\end{array}$ \\
\hline & 3 & 49.67 & $\begin{array}{c}\text { Radial/median crack arrested by interface; } \\
\text { lateral crack present }\end{array}$ \\
\hline & 4 & 52.16 & $\begin{array}{l}\text { Radial/median crack arrested by interface; } \\
\text { lateral crack present }\end{array}$ \\
\hline \multirow{4}{*}{720} & 1 & 47.11 & $\begin{array}{l}\text { Radial/median crack arrested by interface; } \\
\text { lateral crack present }\end{array}$ \\
\hline & 2 & 44.08 & $\begin{array}{l}\text { Radial/median crack arrested by interface; } \\
\text { lateral crack present }\end{array}$ \\
\hline & 3 & 45.11 & $\begin{array}{l}\text { Radial/median crack arrested by interface; } \\
\text { lateral crack present }\end{array}$ \\
\hline & 4 & 49.88 & $\begin{array}{l}\text { Radial/median crack arrested by interface; } \\
\text { lateral crack present }\end{array}$ \\
\hline
\end{tabular}




\subsection{Summary of Experimental Work}

Chapter 4 presents the experimental details conducted to verify the key assumptions made for developing the fracture toughness model in Chapter 3. As a result of post-indentation examination on the cross-sections of the $\mathrm{WC} / 10 \mathrm{Co} / 4 \mathrm{Cr}$ coating $/ 1080$ low carbon steel substrate system, it was established that the dominant fracture patterns in the coating were the radial/median cracks. It was also visually confirmed that at all indentation loads the radial/median crack remained confined within the coating layer without penetrating into the substrate, hence confirming the assumption of the crack ellipticity. Additionally, at all tests, the coating layer remained well-bonded to the substrate layer.

The indentation tests were also used to collect the data required for further validation of the developed fracture toughness model, as would be presented in Chapter 5. As a result of after-polishing coating measurement with a scanning electron microscope, an average thickness of the $\mathrm{WC} / 10 \mathrm{Co} / 4 \mathrm{Cr}$ coating was determined as $45.3 \mu \mathrm{m}$. The radial crack lengths and the indentation mark diagonals were also collected for the $\mathrm{WC} / 10 \mathrm{Co} / 4 \mathrm{Cr}$ coating under different indentation loads and these data are summarized in Table 4-1 and Table 4-2 respectively. Using the Vickers indenters, the hardness values of the coating material and substrate material were also determined; they are $H_{c}=10.4 \mathrm{GPa}$ and $H_{s}=1.05 \mathrm{GPa}$ respectively. 


\section{Chapter 5. Model Verification and Discussion}

\subsection{Proposed Fracture Toughness Model}

\subsubsection{Outline of Verification Tasks}

It has been demonstrated in the indentation tests described in Chapter 4 that all indentation-generated radial/median cracks developed within the coating layer of the $\mathrm{WC} / 10 \mathrm{Co} / 4 \mathrm{Cr}$ coating/1080 steel substrate system. Since the observed radial crack lengths were much longer than the coating thickness, it is reasonable to assume that radial/median cracks are semi-elliptical in shape.

Chapter 5 presents a verification procedure of the analytical approach, or the fracture toughness model proposed in Chapter 3, which is utilized to assess fracture toughness of brittle coating/ductile substrate systems. To accomplish that, the analytical solution derived in Chapter 3, pertinent to the case of the HVOF $\mathrm{WC} / 10 \mathrm{Co} / 4 \mathrm{Cr}$ coating/1080 low carbon steel substrate system tested in Chapter 4 , is utilized to determine the fracture toughness of the brittle coating/ductile substrate system. The key parameters in the model, including the crack shape ellipticity factor $k$, the crack shape influence factor $\beta$ and the residual factor $\chi_{r}$ are evaluated first and then used to calculate the fracture toughness $K_{c}$ of the coating/substrate system in question. The obtained fracture toughness value is validated against the known fracture toughness value of a similar brittle coating/ductile substrate system (HVOF WC/12Co coating/1020 low carbon steel substrate), determined by Lima et al. [38]. Furthermore, the fracture toughness value determined with the proposed model and the fracture toughness values calculated with the existing models by Lawn et al. [11] and Smith and Scattergood [21] 
are compared to highlight the significant improvement in the fracture toughness assessment of brittle coating/ductile substrate systems made in the proposed model. A detailed discussion regarding the advantages and limitations of the proposed fracture toughness model is also provided in this Chapter.

\subsubsection{Relevant Considerations for the Proposed Model}

In the present analysis, for the case of the $\mathrm{WC} / 10 \mathrm{Co} / 4 \mathrm{Cr}$ coating $/ 1080$ steel substrate system, the fracture toughness is to be evaluated for the radial (surface) cracks observed in the specimen after the complete indenter removal. As confirmed by the FIB examination in Chapter 4, in the $\mathrm{WC} / 10 \mathrm{Co} / 4 \mathrm{Cr}$ coating/1080 steel substrate system, the elliptical crack propagated downwards and was always confined within the coating layer whether the indentation load was lower or higher. Moreover, at all indentation loads a radial crack propagated through almost the entire coating thickness, i.e., a radial/median crack propagated much deeper than $7 \%$ of the coating thickness $h_{c}$ but was still confined within it. This means that as a first approximation for all the following calculations, the crack depth $a$ may be approximated as an average coating thickness $h_{c}$. It also implies that the fracture toughness of the post-indentation radial cracks developed in the $\mathrm{WC} / 10 \mathrm{Co} / 4 \mathrm{Cr}$ coating/1080 steel substrate system is to be evaluated based on the solution to the case II of the crack configuration $\left(0.07 h_{c}<a<h_{c}\right)$, described in Section 3.3.2. These radial cracks are supposed to be driven open by the residual stress intensity factor, as defined by Eq. (3-10 a), which arises from the plastic zone expansion under indentation. Generally, as Eq. (3-10 a) shows, the crack driving force $K_{r}$ decreases with an increasing radial crack length $c$, and the crack arrests when $K_{r}$ drops 
below the fracture toughness $K_{c}$ of the coating. At the surface points, corresponding to locations of the radial cracks, $\varphi=0$ or $\pi$, see Figure 3-1 b. After substituting these values into Eq. (3-9 d), an elliptical function $\Phi(0, k)=1$. It is also important to note that according to Eq. (3-25) at the surface points $\varphi=0$ or $\varphi=\pi$, that is at $\xi=\pi$ or $\xi=2 \pi$ in Eq. (3-25), the interface correction factor $Y_{I a}$ is equal to 1 . This demonstrates that the stress intensity factor evaluated at the surface points of the crack is not influenced by the interface. Thus, the influence of the interface through the interface correction factor derived in Section 3.3.2 is not incorporated into the analysis of the radial cracks in the $\mathrm{WC} / 10 \mathrm{Co} / 4 \mathrm{Cr}$ coating/1080 steel substrate system. Therefore, Eq. (3-10 a) yields to the following relationship for the observed radial cracks in the $\mathrm{WC} / 10 \mathrm{Co} / 4 \mathrm{Cr}$ coating $/ 1080$ steel substrate system:

$$
K_{r}=\beta \cdot \chi_{r} \cdot \frac{P}{c^{3 / 2}} \quad(\text { for } \varphi=0 \text { or } \varphi=\pi)
$$

Since the elastic component diminishes completely at the unloading (i.e., the stress intensity factor corresponding to the elastic stress component diminishes to zero $\left(K_{e}=0\right)$ ), and the residual component persists upon indenter removal, the post-indentation radial cracks are governed by the stress intensity factor corresponding to the residual stress component only. Hence, for post-indentation radial cracks in the coating/substrate system in question, the stress intensity factor corresponding to the residual stress component $K_{r}$ is equal to the fracture toughness $K_{c}$, as per Eq. (3-17). Thus, Eq. (5-1 a) can be re-written as: 


$$
K_{c}=\beta \cdot \chi_{r} \cdot \frac{P}{c^{3 / 2}} \quad(\text { for } \varphi=0 \text { or } \varphi=\pi)
$$

In the meanwhile, the crack shape influence factor $\beta$ is determined by Eqs. (3-9 b) to (3-9 e) as follows:

$$
\begin{aligned}
& \beta=\frac{\pi}{2\left(1-k^{2}\right)^{1 / 2} \cdot L(k)} \\
& L(k)=\int_{0}^{\pi / 2} \Phi(\varphi, k) \cdot\left(1+\frac{k^{2}}{1-k^{2}} \cdot \sin ^{2} \varphi\right)^{1 / 4} d \varphi \\
& \Phi(\varphi, k)=\left(1+\frac{k^{2}}{1-k^{2}} \cdot \sin ^{2} \varphi\right)^{1 / 4} \cdot\left(1-k^{2} \cdot \sin ^{2} \varphi\right)^{-1} \\
& k^{2}=1-\left(\frac{a}{c}\right)^{2}
\end{aligned}
$$

and the residual factor $\chi_{r}$ is defined according to Eq. (3-16) as follows:

$$
\chi_{r}=\frac{1}{3 \pi^{2} \cdot\left(1-2 v_{c}\right)} \cdot\left(\frac{E_{c}}{H_{m}}\right)^{1 / 2} \cdot(\cot \psi)^{2 / 3}
$$

To calculate the fracture toughness $K_{c}$ for the $\mathrm{WC} / 10 \mathrm{Co} / 4 \mathrm{Cr}$ coating/ 1080 steel substrate system according to Eq. (5-1 b), the average values of the radial crack lengths $c_{\text {exp }}$ corresponding to each indentation load level, summarized in Table 4-1 in Chapter 4, are used. The crack shape influence factor $\beta$ values are also required in order to obtain the fracture toughness $K_{c}$. To proceed, firstly, the crack shape ellipticity factor $k$ is calculated with Eq. (3-9 e). Secondly, the $\beta$ values are calculated using the $k$ values in terms of Eqs. (3-9 b) to (3-9 d). Thirdly, the residual factor $\chi_{r}$ values are computed according to Eq. (3-16), but prior to this, the composite hardness values $H_{m}$ are 
determined with Eq. (3-15). Finally, the fracture toughness values $K_{c}$ can be obtained in terms of Eq. (5-1 b). The following sections will describe the details of these calculations.

\subsubsection{Evaluation of Key Parameters in the Proposed Model}

\subsubsection{Crack Shape Ellipticity Factor $k$}

To evaluate the crack shape influence factor $\beta$ in Eq. (5-1 b), the crack shape ellipticity factor $k$ should be calculated first. Using Eq. (3-9 e), $k=\sqrt{1-(a / c)^{2}}$. The $a$ value is equal to the coating thickness $h_{c}$, as explained above, and the average radial crack lengths $c_{\text {exp }}$ are based on test data in Table 4-1 in Chapter 4. The calculated $k$ values are summarized in Table 5-1.

Table 5-1. A summary of the crack shape ellipticity factor $k$ values.

\begin{tabular}{|c|c|c|c|c|}
\hline Load $P(\mathrm{~N})$ & $\begin{array}{c}\text { Average radial crack } \\
\text { length } c_{\exp }(\mu \mathrm{m})\end{array}$ & $\begin{array}{l}\text { Crack depth } \\
a\left(\approx h_{c}\right)(\mu \mathrm{m})\end{array}$ & $k^{2}=1-(a / c)^{2}$ & $k$ \\
\hline 196 & 497.700 & \multirow{5}{*}{45.3} & 0.9917 & 0.9958 \\
\hline 294 & 622.330 & & 0.9947 & 0.9973 \\
\hline 392 & 686.625 & & 0.9956 & 0.9978 \\
\hline 490 & 727.875 & & 0.9961 & 0.9980 \\
\hline 720 & 859.100 & & 0.9972 & 0.9986 \\
\hline
\end{tabular}

It is shown in Table 5-1 that within the scope of the experimental work (i.e., within the range of the indentation loads used in this research), the $k$ values range between 0.9958 and 0.9986 . In addition, since the radial crack length $c$ increases with the indentation load, the crack shape ellipticity factor $k$ values vary for different indentation loads, according to Eq. (3-9 e). 


\subsubsection{Crack Shape Influence Factor $\beta$}

The crack shape influence factor $\beta$ values are calculated using Eqs. (3-9 b) to (3-9 d). These equations were programmed with the engineering calculation software MathCAD. To ensure accuracy of the calculations with this software, the $\beta$ values from MathCAD were compared with the known $\beta$ values from Borodachev's work [18] for $k$ ranging from 0 to 0.9 . An example calculation of the crack shape influence factor $\beta$ in MathCAD for $k=0.1$ is presented below.

For $k=0.1(\varphi=0$ or $\varphi=\pi)$

a) $\Phi(\varphi, k):=\left(1+\frac{0.1^{2}}{1-0.1^{2}} \cdot \sin ^{2} \varphi\right)^{1 / 4} \cdot\left(1-0.1^{2} \cdot \sin ^{2} \varphi\right)^{-1}$

b) $L(k)=\int_{0}^{\pi / 2} \Phi(\varphi, 0.1) \cdot\left(1+\frac{0.1^{2}}{1-0.1^{2}} \cdot \sin ^{2} \varphi\right)^{1 / 4} d \varphi=1.583$

c) $\beta:=\frac{\pi}{2 \cdot\left(1-0.1^{2}\right)^{1 / 2} \cdot 1.583}$

d) $\beta=0.9972$

An example calculation of the $\beta$ value in MathCAD for $k=0.9958$ is demonstrated below.

For $k=0.9958(\varphi=0$ or $\varphi=\pi)$

a) $\Phi(\varphi, k):=\left(1+\frac{0.9958^{2}}{1-0.9958^{2}} \cdot \sin ^{2} \varphi\right)^{1 / 4} \cdot\left(1-0.9958^{2} \cdot \sin ^{2} \varphi\right)^{-1}$

b) $L(k)=\int_{0}^{\pi / 2} \Phi(\varphi, 0.9958) \cdot\left(1+\frac{0.9958^{2}}{1-0.9958^{2}} \cdot \sin ^{2} \varphi\right)^{1 / 4} d \varphi=177.389$

c) $\beta:=\frac{\pi}{2 \cdot\left(1-0.9958^{2}\right)^{1 / 2} \cdot 177.389}$ 
d) $\beta=0.0967$

All the $\beta$ values for $k$ ranging from 0 to 0.9986 are calculated in MathCAD similarly with the calculations presented above and are summarized in Table 5-2, along with the known $\beta$ values from Borodachev's work for $k$ ranging between 0 and 0.9 for comparison.

Table 5-2. A summary of the crack shape influence factor $\beta$ values.

\begin{tabular}{|c|c|c|}
\hline$k$ & $\beta$ (Borodachev's work) & $\beta$ (MathCAD) \\
\hline 0 & 1 & 0.9972 \\
\hline 0.1 & 0.998 & 0.9754 \\
\hline 0.3 & 0.976 & 0.9221 \\
\hline 0.5 & 0.922 & 0.8054 \\
\hline 0.7 & 0.806 & 0.6966 \\
\hline 0.8 & 0.697 & 0.5123 \\
\hline 0.9 & 0.512 & 0.0970 \\
\hline 0.9958 & N/A & 0.0810 \\
\hline 0.9970 & N/A & 0.0710 \\
\hline 0.9978 & N/A & 0.0657 \\
\hline 0.9980 & N/A & 0.0540 \\
\hline 0.9986 & N/A & 0.06 \\
\hline
\end{tabular}

It is evident from Table 5-2 that the $\beta$ values calculated by Borodachev (for the $k$ values ranging between 0 and 0.9 ) and with MathCAD software for the same range of $k$ values are very close (a percent error between $\beta$ values calculated with MathCAD and by Borodachev is less than $1 \%$ for $k$ values ranging between 0 and 0.9 ). This confirms accuracy of the MathCAD calculations. Thus, the obtained with MathCAD software $\beta$ values for $k$ values ranging from 0.9958 to 0.9986 are proved to be accurate. 
The crack shape influence factor $\beta$ values, calculated with MathCAD software, are further plotted as a function of the crack shape ellipticity factor $k$, as shown in Figure 5-1. Since the $k$ parameter varies with the indentation load, the $\beta$ parameter is a function of the indentation load as well.

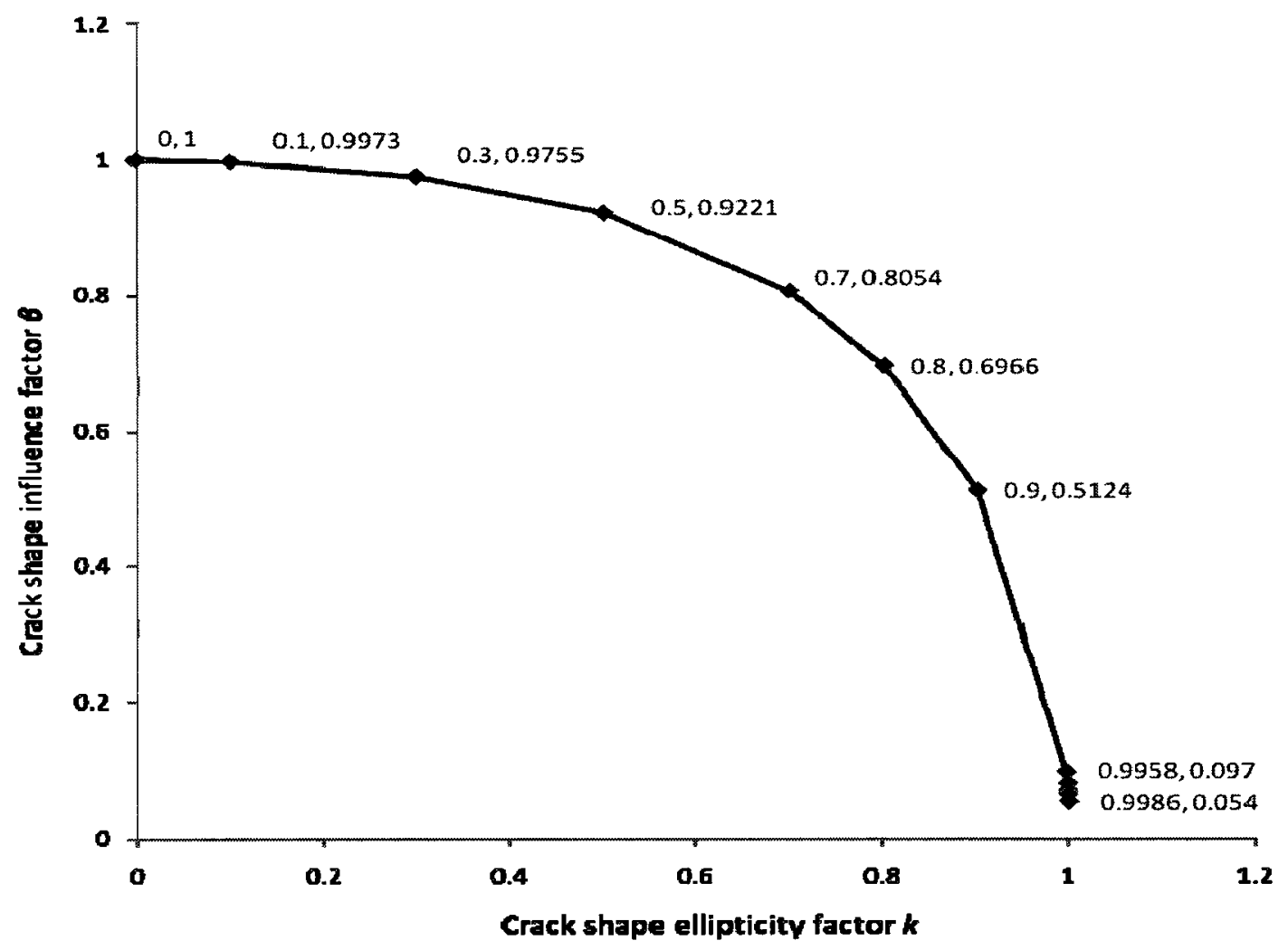

Figure 5-1. Crack shape influence factor $\beta$ vs. the crack shape ellipticity factor $k$.

It is evident from Figure 5-1 that for all average radial crack lengths $c_{\text {exp }}$ developed during the indentation tests in $\mathrm{WC} / 10 \mathrm{Co} / 4 \mathrm{Cr}$ coating/1080 steel substrate system, the crack shape ellipticity factor $k$ approaches 1 . This can be attributed to the average radial crack lengths $c_{\text {exp }}$ being much longer than the coating depth $h_{c}$ (which is used as an approximation to the crack depths $a$ ), according to Eq. (3-9 e). 


\subsubsection{Residual Factor $\chi_{r}$ Corresponding to Residual Stress Component}

As described in Section 3.3.2, the residual factor $\chi_{r}$ is defined with Eq. (3-16) as:

$\chi_{r}=\frac{1}{3 \pi^{2}\left(1-2 v_{c}\right)} \cdot\left(\frac{E_{c}}{H_{m}}\right)^{1 / 2} \cdot(\cot \psi)^{2 / 3}$

where the composite hardness $H_{m}$ of the coating/substrate system may be derived according to Eq. (3-15) as [55]:

$H_{m}=3\left(\frac{h_{c}}{2 l}\right)\left(\frac{H_{c}}{E_{c}}\right)^{1 / 2} \cdot\left(H_{c}-H_{s}\right)(\tan \psi)^{1 / 3}+H_{s}$

Therefore, to calculate the residual factor $\chi_{r}$ values, the composite hardness $H_{m}$ values should be determined first. In Eq. (3-15), the indentation diagonals $l$ have been obtained for the $\mathrm{WC} / 10 \mathrm{Co} / 4 \mathrm{Cr}$ coating/1080 steel substrate system from the indentation tests, as reported in Table 4-2 in Chapter 4. Taking the average values of the indentation diagonals $l$ of each indentation mark and utilizing the coating and substrate material properties as well as the indenter angle summarized in Table 5-3, the composite hardness values $H_{m}$ of the $\mathrm{WC} / 10 \mathrm{Co} / 4 \mathrm{Cr}$ coating/1080 steel substrate system were calculated for each indentation test at different indentation loads. These values are summarized in Table 5-4.

Table 5-3. A summary of the material parameters of the

WC/10Co/4Cr coating/1080 steel substrate system.

\begin{tabular}{|c|c|c|c|c|c|c|}
\hline$h_{c}(\mu \mathrm{m})$ & $H_{c}(\mathrm{GPa})$ & $E_{c}(\mathrm{GPa})$ & $\nu_{c}$ & $E_{s}(\mathrm{GPa})$ & $H_{s}(\mathrm{GPa})$ & $\psi(\mathrm{rad})$ \\
\hline 45.3 & 10 & 310 & 0.3 & 250 & 1.056 & 1.1868 \\
\hline
\end{tabular}


Table 5-4. A summary of the composite hardness $H_{m}$ values.

\begin{tabular}{|c|c|c|}
\hline Load $P(\mathrm{~N})$ & $\begin{array}{c}\text { Average indentation diagonal } l \\
(\mu \mathrm{m})\end{array}$ & $\begin{array}{c}\text { Composite hardness } H_{m} \\
\text { (GPa) }\end{array}$ \\
\hline \multirow{3}{*}{196} & 616.6 & 1.529 \\
\hline & 73.5 & 1.450 \\
\hline & 628.5 & 1.520 \\
\hline \multirow{3}{*}{294} & 813.0 & 1.413 \\
\hline & 831.5 & 1.405 \\
\hline & 831.0 & 1.405 \\
\hline \multirow{3}{*}{392} & 852.5 & 1.396 \\
\hline & 881.5 & 1.385 \\
\hline & 877.0 & 1.387 \\
\hline \multirow{3}{*}{490} & 918.0 & 1.371 \\
\hline & 921.5 & 1.370 \\
\hline & 96.5 & 1.372 \\
\hline \multirow{3}{*}{720} & 956.5 & 1.359 \\
\hline & 949.0 & 1.361 \\
\hline & 959.5 & 1.357 \\
\hline
\end{tabular}

Taking the average composite hardness value $H_{m}$ and utilizing Table 5-3 for each indentation load $P$, the residual factor $\chi_{r}$ values for the $\mathrm{WC} / 10 \mathrm{Co} / 4 \mathrm{Cr}$ coating/1080 steel substrate system were calculated in terms of Eq. (3-16), as summarized in Table 5-5. Since the composite hardness $H_{m}$ varies with the 
indentation load, the $\chi_{r}$ parameter is a function of the indentation load $P$, according to Eq. (3-16).

Table 5-5. A summary of the residual factor $\chi_{r}$ values.

\begin{tabular}{|c|c|c|}
\hline Load $P(\mathrm{~N})$ & $\begin{array}{c}\text { Average composite hardness } \\
H_{m} \text { per load } P(\mathrm{GPa})\end{array}$ & Residual factor $\chi_{r}$ \\
\hline 196 & 1.500 & 0.781 \\
\hline 294 & 1.408 & 0.806 \\
\hline 392 & 1.389 & 0.811 \\
\hline 490 & 1.371 & 0.816 \\
\hline 720 & 1.359 & 0.820 \\
\hline
\end{tabular}

\subsubsection{Determination of Fracture Toughness with the Proposed Model}

Upon the parameters $\beta$ and $\chi_{r}$ available in Table 5-2 and Table 5-5 respectively, the fracture toughness of the $\mathrm{WC} / 10 \mathrm{Co} / 4 \mathrm{Cr}$ coating/1080 steel substrate system is determined using Eq. (5-1 b), based on the experimental data of the average radial crack lengths $c_{\text {exp }}$ corresponding to the indentation loads $P$ in Table 5-1. The calculated fracture toughness $K_{c}$ values and all the values that are used to calculate the fracture toughness with the proposed model are summarized in Table 5-6. 
Table 5-6. Fracture toughness values based on the proposed model.

\begin{tabular}{|c|c|c|c|c|}
\hline Load $P(\mathrm{~N})$ & $\beta$ & $\begin{array}{c}\text { Radial crack length } \\
c_{\text {exp }}^{3 / 2}(\mathrm{~m})^{3 / 2}\end{array}$ & $\begin{array}{c}\text { Residual } \\
\text { factor } \chi_{r}\end{array}$ & $\begin{array}{c}\text { Fracture toughness } \\
K_{c}\left(\mathrm{MPa} \mathrm{m}^{1 / 2}\right)\end{array}$ \\
\hline 196 & 0.0970 & $1.11 \mathrm{E}-05$ & 0.781 & 1.34 \\
\hline 294 & 0.0810 & $1.55 \mathrm{E}-05$ & 0.806 & 1.24 \\
\hline 392 & 0.0710 & $1.80 \mathrm{E}-05$ & 0.811 & 1.26 \\
\hline 490 & 0.0657 & $1.96 \mathrm{E}-05$ & 0.816 & 1.34 \\
\hline 720 & 0.0540 & $2.52 \mathrm{E}-05$ & 0.820 & 1.27 \\
\hline
\end{tabular}

The fracture toughness values $K_{c}$ from Table 5-6 are plotted against the indentation load $P$ in Figure 5-2.

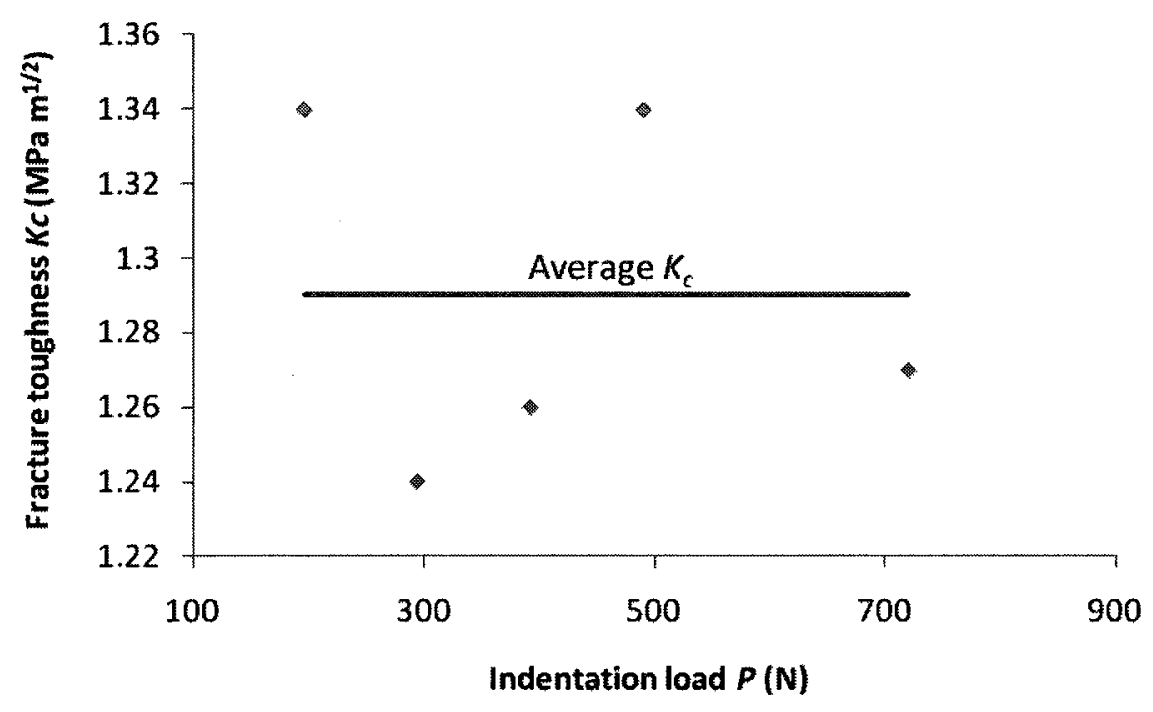

Figure 5-2. Fracture toughness values based on the proposed model.

It is obvious from the graph that the fracture toughness values calculated at five load levels are close to a constant (an average value), which is $K_{c}=1.29 \mathrm{MPa} \mathrm{m}{ }^{1 / 2}$. The variation range of the fracture toughness is about $\pm 0.05 \mathrm{MPa} \mathrm{m}^{1 / 2}$. This variation range is very small, compared with the wide range of the indentation 
load $P(196 \mathrm{~N} \sim 720 \mathrm{~N})$; hence, the fracture toughness is almost constant. It is well known that the fracture toughness is a material property or a property of material systems, such as coating/substrate systems. Hence, it is independent of the indentation load. The fracture toughness for the $\mathrm{WC} / 10 \mathrm{Co} / 4 \mathrm{Cr}$ coating/1080 steel substrate system, determined with the proposed fracture toughness model, exhibits such a behavior.

As reported in 2.2.1.1, Lima et al. determined the fracture toughness $K_{c}=1.6 \pm 0.9 \mathrm{MPa} \mathrm{m}{ }^{1 / 2}$ for a high-velocity oxy-fuel (HVOF) WC/12Co coating on a 1020 low carbon steel substrate system [38]. It is apparent that (HVOF) WC/12Co coating/1020 steel substrate system is very similar to (HVOF) $\mathrm{WC} / 10 \mathrm{Co} / 4 \mathrm{Cr}$ coating/1080 steel substrate system due to similarity in coating chemical composition and deposition technique as well as similarity between the substrate materials. Furthermore, if the $H / E$ ratios (where $H$ is hardness and $E$ is Young's modulus) are compared for each coating/substrate system (these ratios are typically used in design of the coating/substrate systems that are resilient to impact wear [38]), they are also found to be very close, as shown in Table 5-7. In general, the higher the $H / E$ ratio, the higher is the resilience (or an ability to absorb elastic energy) of the coating/substrate system to impact wear. 
Table 5-7. Ratios of hardness to Young's modulus of the

two similar coating/substrate systems.

\begin{tabular}{|c|c|c|}
\hline Ratio & $H_{c} / E_{c}$ & $H_{s} / E_{s}$ \\
\hline WC/10Co/4Cr coating/1080 steel substrate & 0.033 & 0.004 \\
\hline WC/12Co coating/1020 steel substrate & 0.044 & 0.005 \\
\hline
\end{tabular}

Therefore, it is reasonable to assume that the fracture toughness value determined by Lima et al. for the (HVOF) WC/12Co coating/1020 steel substrate system is comparable to that of the (HVOF) $\mathrm{WC} / 10 \mathrm{Co} / 4 \mathrm{Cr}$ coating/1080 steel substrate system. In other words, the fracture toughness of $1.29 \pm 0.05 \mathrm{MPa} \mathrm{m}^{1 / 2}$ calculated with the proposed model is a realistic value for the $\mathrm{WC} / 10 \mathrm{Co} / 4 \mathrm{Cr}$ coating/1080 steel substrate system, because it is within the same order of magnitude with the value of $1.60 \pm 0.90 \mathrm{MPa} \mathrm{m}{ }^{1 / 2}$ provided by Lima et al. The percent error between the average fracture toughness value estimated using Eq. (5-1 b), i.e., $1.29 \mathrm{MPa} \mathrm{m}^{1 / 2}$, and the value of $1.60 \mathrm{MPa} \mathrm{m}^{1 / 2}$ provided by Lima et al., yields about $19 \%$. This difference is acceptable since the compared coating/substrate systems are similar but not identical.

\subsubsection{Response Behaviour of Crack Length to Indentation Load}

In terms of Eq. (5-1 b), we can write:

$$
c^{3 / 2}=\beta \cdot \frac{\chi_{r}}{K_{c}} P
$$


Based on Eqs. (3-9 b) - (3-9 e) and Eq. (3-16), both values $\beta$ and $\chi_{r}$ vary with the indentation load; that is, $\beta \cdot \frac{\chi_{r}}{K_{c}}$ is a variable, not a constant. This means that $c^{3 / 2}$ vs. $P$ exhibits a non-linear relationship according to Eq. (5-1 c).

To confirm this behavior, radial crack lengths $c^{3 / 2}$ vs. applied indentation load $P$, are plotted in Figure 5-3 in terms of the proposed model (see Eq. (5-1 c)). To obtain values of the radial crack lengths $c^{3 / 2}, \beta$ and $\chi_{r}$ values are adopted from Table 5-6 and an average fracture toughness of $1.29 \mathrm{MPa} \mathrm{m}^{1 / 2}$ is assumed for the $\mathrm{WC} / 10 \mathrm{Co} / 4 \mathrm{Cr}$ coating/1080 steel substrate system, as validated in Section 5.1.4. Experimental data points in terms of the radial crack lengths $c^{3 / 2}$ from Table 4-1 are also plotted on the same graph (Figure 5-3) to outline a non-linear trend in radial crack length $c^{3 / 2}$ behavior vs. applied indentation load $P$.

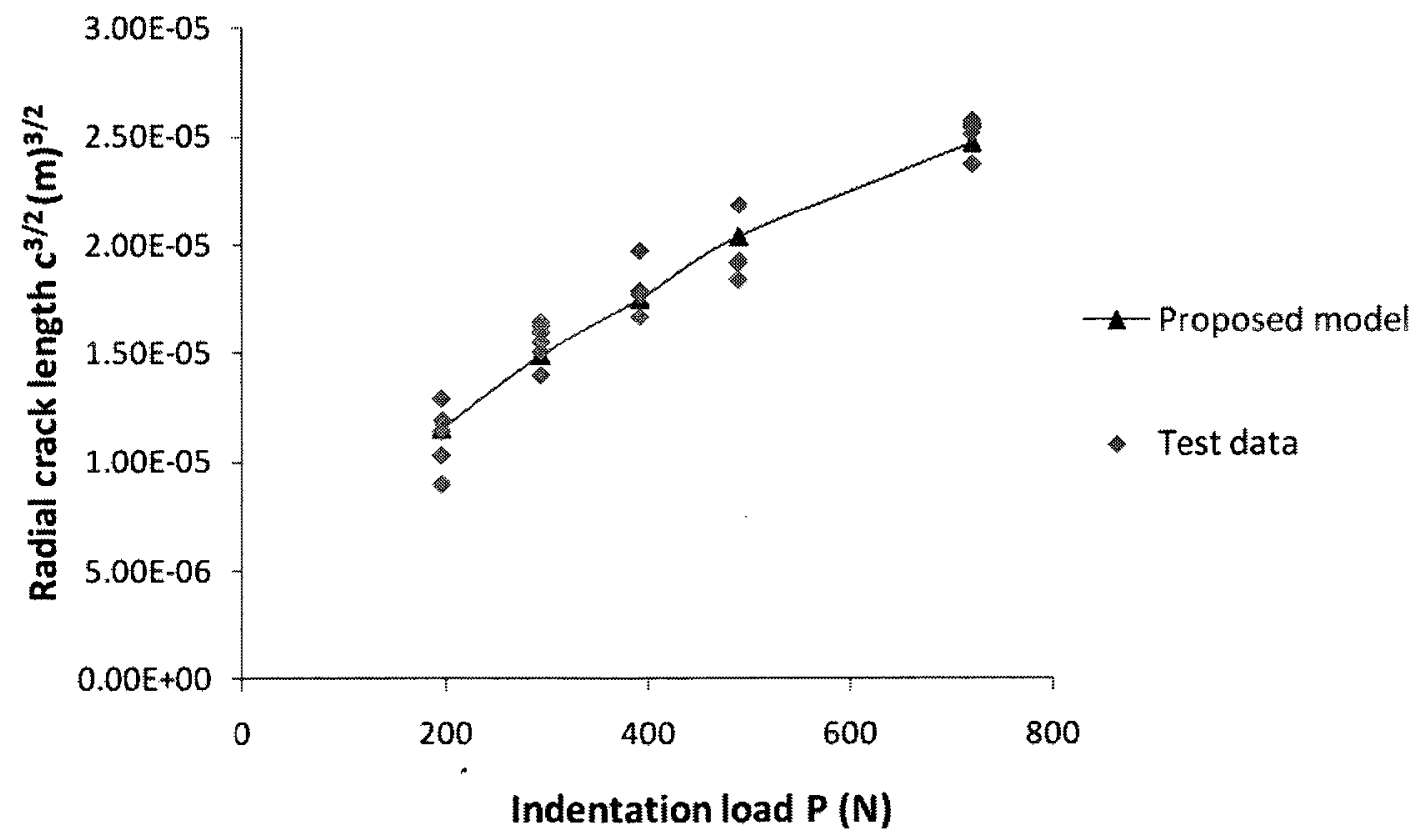

Figure 5-3. Non-linear behavior of radial crack length $c^{3 / 2}$ vs. indentation load $P$. 
It is evident from Figure 5-3 that the indentation tests demonstrate the non-linear behavior between the radial crack length $c^{3 / 2}$ vs. the indentation load $P$, which is consistent with the proposed analytical model in terms of Eq. (5-1 c). The radial crack length $c^{3 / 2}$ increases with the indentation load $P$ non-linearly. This may be attributed to the change in the crack shape, i.e., an increase in the crack ellipticity as the indentation load $P$ increases.

\subsection{Comparison between the Proposed Model and Other}

\section{Existing Models}

\subsubsection{Smith-Scattergood Fracture Toughness Model}

As detailed in Section 2.2.1.1, Smith and Scattergood considered the crack shape as an influential factor on the indentation fracture toughness for monolithic brittle materials under indentation [21]. The authors assumed that both the radial/median and Palmqvist cracks might form in a semi-elliptical shape; thus, the authors derived a correction factor for the radial cracks based on the empirical weight function method [21] such that for the residual component,

$K_{c}=F_{\text {res }} \cdot \frac{\chi_{r} P}{c^{3 / 2}}$ (neglecting free surface effect)

where $c$ is a radial crack length.

$F_{\text {res }}=f_{\text {res }} \cdot g_{\text {res }}$

$f_{\text {res }}=\left(\frac{1}{\pi} \int_{0}^{\pi} \frac{\left(1-k^{2} \cos ^{2} \varphi\right)^{1 / 2}}{1-k^{2} \sin ^{2} \varphi} d \varphi\right)^{-1 / 2}(\varphi=0$ or $\varphi=\pi)$

and $g_{\text {res }} \approx 1.22$ according to the work of Smith and Scattergood [21]. 
To compare the proposed fracture toughness model to Smith-Scattergood model, the $f_{\text {res }}$ values corresponding to the same range of indentation loads $P$ (Table 5-6) and thereby, the same values of the crack shape ellipticity factor $k$ (Table 5-1) had to be calculated first. To accomplish that, the $f_{\text {res }}$ values were programmed with the engineering software MathCAD. An example calculation of the $f_{\text {res }}$ factor for the $k$ value of 0.0958 is demonstrated below.

For $k=0.9958(\varphi=0$ or $\varphi=\pi)$

$$
f_{\text {res }}=\left[\frac{1}{\pi} \int_{0}^{\pi} \frac{\left(1-0.9958^{2} \cdot \cos ^{2} \varphi\right)^{1 / 2}}{1-0.9958^{2} \cdot \sin ^{2} \varphi} d \varphi\right]^{-1 / 2}=0.311
$$

A summary of the $f_{\text {res }}$ values (calculated in MathCAD similarly with the calculation presented above) corresponding to the crack shape ellipticity factor $k$ values ranging from 0.0958 to 0.9986 is provided in Table 5-8.

Table 5-8. A summary of $f_{\text {res }}$ values corresponding to $k$ values.

\begin{tabular}{|c|c|}
\hline$k$ & $f_{\text {res }}$ \\
\hline 0.0958 & 0.311 \\
\hline 0.9970 & 0.285 \\
\hline 0.9978 & 0.263 \\
\hline 0.9980 & 0.256 \\
\hline 0.9986 & 0.234 \\
\hline
\end{tabular}

To calculate the fracture toughness of the $\mathrm{WC} / 10 \mathrm{Co} / 4 \mathrm{Cr}$ coating/1080 steel substrate with Smith-Scattergood model, i.e., with Eq. (5-2 a), the residual factor $\chi_{r}$ values calculated previously in Section 5.1.3.3 and summarized in Table 5-6, are re-used (in the original work of Smith-Scattergood [21], the residual factor $\chi_{r}$ is 
determined purely from tests as a calibration constant to the experimental data; there is no analytical expression for this constant). Since the $f_{\text {res }}$ values and the residual factor $\chi_{r}$ values are available, and the average radial crack lengths $c_{\text {exp }}$ corresponding to the indentation loads $P$ were determined from the indentation tests (Table 5-1), the fracture toughness $K_{c}$ values can be established according to Eq. (5-2 a). The calculated fracture toughness $K_{c}$ values and all the values that are used to calculate these fracture toughness values are summarized in Table 5-9.

Table 5-9. Fracture toughness values based on Smith-Scattergood model.

\begin{tabular}{|c|c|c|c|c|c|c|}
\hline Load $P(\mathrm{~N})$ & $k$ & $f_{\text {res }}$ & $g_{\text {res }}$ & $\begin{array}{l}\text { Radial crack } \\
\text { length } c_{\text {exp }}^{3 / 2} \\
(\mathrm{~m})^{3 / 2}\end{array}$ & $\begin{array}{c}\text { Residual } \\
\text { factor } \\
\chi_{r}\end{array}$ & $\begin{array}{c}\text { Fracture } \\
\text { toughness } \\
K_{c}\left(\mathrm{MPa} \mathrm{m}^{1 / 2}\right)\end{array}$ \\
\hline 196 & 0.0958 & 0.311 & \multirow{5}{*}{1.22} & $1.11 \mathrm{E}-05$ & 0.781 & 5.23 \\
\hline 294 & 0.9970 & 0.285 & & $1.55 \mathrm{E}-05$ & 0.806 & 5.31 \\
\hline 392 & 0.9978 & 0.263 & & $1.80 \mathrm{E}-05$ & 0.811 & 5.67 \\
\hline 490 & 0.9980 & 0.256 & & $1.96 \mathrm{E}-05$ & 0.816 & 6.37 \\
\hline 720 & 0.9986 & 0.234 & & $2.52 \mathrm{E}-05$ & 0.820 & 6.70 \\
\hline
\end{tabular}

The fracture toughness values $K_{c}$ from Table 5-9 are plotted against the indentation load $P$ in Figure 5-4. 


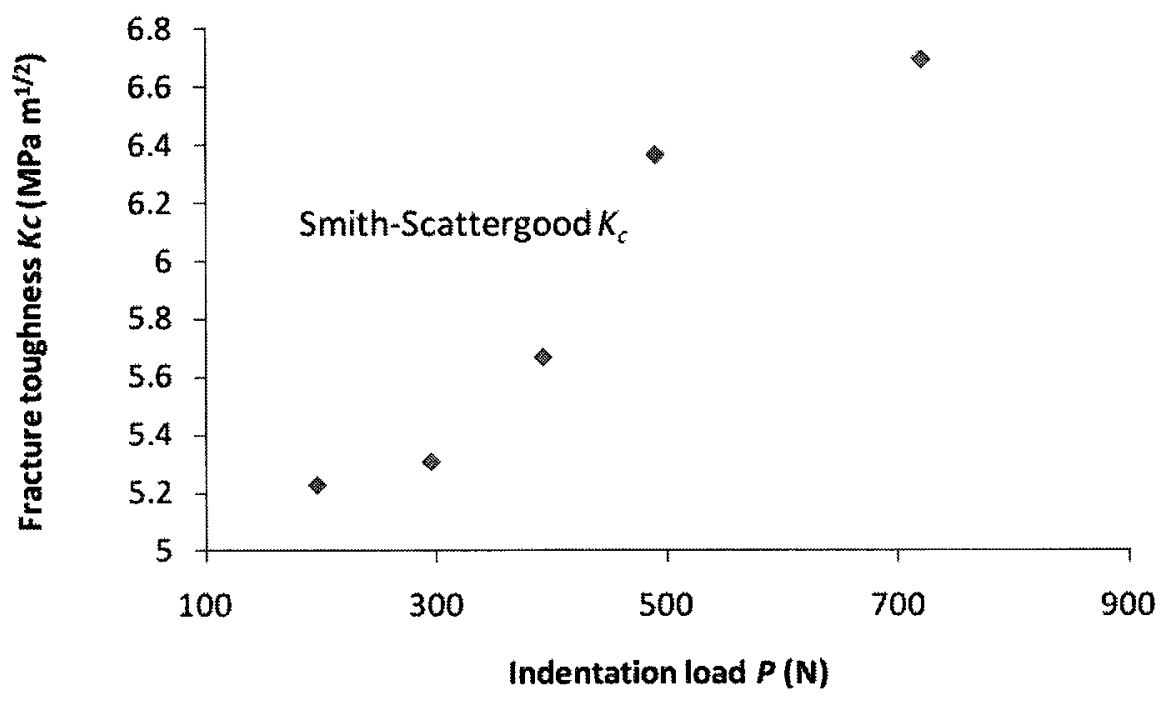

Figure 5-4. Fracture toughness values based on Smith-Scattergood model.

It is obvious from Figure 5-4 that based on Smith-Scattergood model, the fracture toughness $K_{c}$ increases with the indentation load and seems not to approach a constant value. Based on Smith-Scattergood model, the fracture toughness varies as $K_{c}=5.965 \pm 0.735 \mathrm{MPa} \mathrm{m}^{1 / 2}$ at indentation load $P$ ranging from 196 to $720 \mathrm{~N}$ for the WC/10Co/4Cr coating/1080 steel substrate system. Evidently, the fracture toughness predictions made with Smith-Scattergood model at different indentation loads $P$ are more scattered in comparison to the fracture toughness values predicted with the proposed model, i.e., $K_{c}=1.29 \pm 0.05 \mathrm{MPa} \mathrm{m} \mathrm{m}^{1 / 2}$. In the viewpoint of fracture mechanics, the fracture toughness is a material parameter or a material system property (such as coating/substrate system or a joint system), which is independent of the indentation load. Therefore, the reliability of the predictions made with Smith-Scattergood model is doubtful.

Since the fracture toughness values predicted with Smith-Scattergood model increase with the indentation load, it is unreasonable to determine an average fracture 
toughness value. However, even if the lowest value of the fracture toughness range is adopted for comparison purposes, i.e., $K_{c}=5.23 \mathrm{MPa} \mathrm{m}^{1 / 2}$ for the $\mathrm{WC} / 10 \mathrm{Co} / 4 \mathrm{Cr}$ coating/1080 steel substrate system, the fracture toughness value calculated with Smith-Scattergood model is 3.3 times higher than that determined by Lima et al. (i.e., an average fracture toughness value of $K_{c}=1.6 \mathrm{MPa} \mathrm{m}^{1 / 2}$ for the (HVOF) WC/12Co coating/1020 steel substrate system). Moreover, when compared with the average fracture toughness value calculated based on the proposed model, i.e., $K_{c}=1.29 \mathrm{MPa} \mathrm{m}{ }^{1 / 2}$, Smith-Scattergood model over predicts the fracture toughness value by 4 times.

\subsubsection{Lawn et al. Fracture Toughness Model}

The model of Lawn et al. was originally developed for monolithic brittle materials containing half-penny cracks under indentation. According to the model provided by Lawn et al., the fracture toughness $K_{c}$ has the following relationship with the crack size $c_{m}$ and the indentation load $P[11]$ :

$$
K_{c}=\chi_{r} \cdot\left(\frac{P}{c_{m}^{3 / 2}}\right)
$$

To calculate the fracture toughness of the $\mathrm{WC} / 10 \mathrm{Co} / 4 \mathrm{Cr}$ coating $/ 1080$ steel substrate with Lawn et al. model, i.e., with Eq. (5-3), the residual factor $\chi_{r}$ values calculated previously in Section 5.1.3.3 and summarized in Table 5-6 are used again.

Since the average radial crack lengths $c_{\text {exp }}$ corresponding to the indentation loads $P$ are available from the indentation tests, the fracture toughness $K_{c}$ values can be 
computed according to Eq. (5-3). The calculated fracture toughness $K_{c}$ and all the values that are used to calculate these fracture toughness values are summarized in Table 5-10.

Table 5-10. Fracture toughness values based on Lawn et al. model.

\begin{tabular}{|c|c|c|c|}
\hline$P(\mathrm{~N})$ & $\begin{array}{c}\text { Radial crack length } \\
c_{\exp }^{3 / 2}(\mathrm{~m})^{3 / 2}\end{array}$ & Residual factor $\chi_{r}$ & $\begin{array}{c}\text { Fracture toughness } \\
K_{c}\left(\mathrm{MPa} \mathrm{m}^{1 / 2}\right)\end{array}$ \\
\hline 196 & $1.11 \mathrm{E}-05$ & 0.781 & 13.8 \\
\hline 294 & $1.55 \mathrm{E}-05$ & 0.806 & 15.3 \\
\hline 392 & $1.80 \mathrm{E}-05$ & 0.811 & 17.7 \\
\hline 490 & $1.96 \mathrm{E}-05$ & 0.816 & 20.4 \\
\hline 720 & $2.52 \mathrm{E}-05$ & 0.820 & 23.5 \\
\hline
\end{tabular}

The fracture toughness values $K_{c}$ from Table 5-10 are plotted against the indentation load $P$ in Figure 5-5.

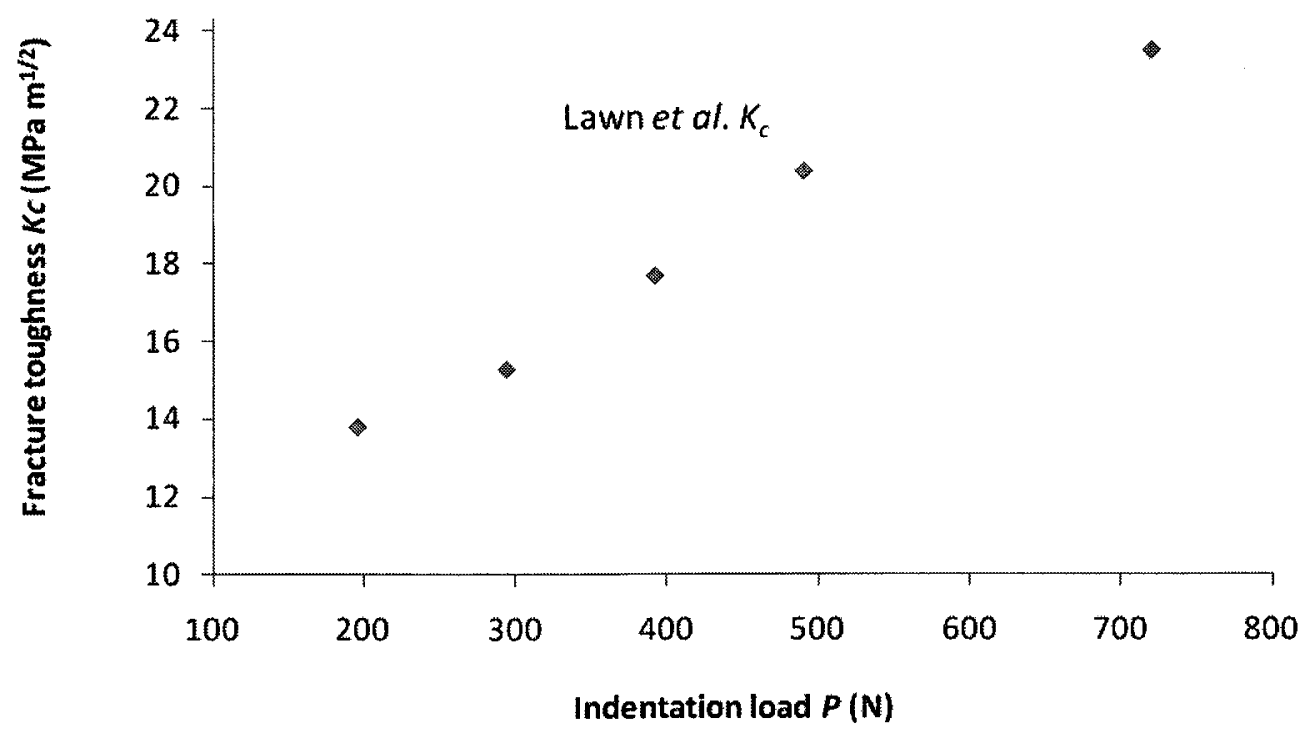

Figure 5-5. Fracture toughness values based on Lawn et al. model. 
It is evident from Figure 5-5 that based on Lawn et al. model, similar to Smith-Scattergood model, the fracture toughness $K_{c}$ increases with the indentation load and seems not to approach a constant value. Based on Lawn et al. model, the fracture toughness varies as $K_{c}=18.7 \pm 4.85 \mathrm{MPa} \mathrm{m}^{1 / 2}$ for the $\mathrm{WC} / 10 \mathrm{Co} / 4 \mathrm{Cr}$ coating/1080 steel substrate system. Evidently, the fracture toughness predictions made with Lawn et al. model at different indentation loads $P$ are largely scattered than those made with the proposed model, i.e., $K_{c}=1.29 \pm 0.05 \mathrm{MPa} \mathrm{m} \mathrm{m}^{1 / 2}$. Again, in the point of fracture mechanics, the fracture toughness is a material parameter or a material system property (such as coating/substrate system or a joint system), which is independent of the indentation load. Hence, the reliability of the predictions made with Lawn et al. model is questionable.

Since the fracture toughness values predicted with Lawn et al. model vary with the indentation load, similar to Smith-Scattergood model, it is unreasonable to determine an average fracture toughness value based on Lawn et al. model. However, even if the lowest value from the range of the fracture toughness values is adopted for comparison purposes, i.e., $K_{c}=13.8 \mathrm{MPa} \mathrm{m} \mathrm{m}^{1 / 2}$ for the $\mathrm{WC} / 10 \mathrm{Co} / 4 \mathrm{Cr}$ coating $/ 1080$ steel substrate system, the fracture toughness value calculated with Lawn et al. model is 8.6 times higher than that determined by Lima et al. (i.e., an average fracture toughness value of $K_{c}=1.6 \mathrm{MPa} \mathrm{m}{ }^{1 / 2}$ for the (HVOF) WC/12Co coating/1020 steel substrate system). Moreover, when compared with the fracture toughness value calculated based on the proposed model, i.e., $K_{c}=1.29 \mathrm{MPa} \mathrm{m}{ }^{1 / 2}$, Lawn et al. model significantly over predicts the fracture toughness value by 11 times. 


\subsubsection{Comparison between the Three Models}

The fracture toughness values estimated with the proposed model, i.e., Eq. (5-1 b), Smith-Scattergood model, i.e., Eq. (5-2 a), and Lawn et al. model, i.e., Eq. (5-3), for the $\mathrm{WC} / 10 \mathrm{Co} / 4 \mathrm{Cr}$ coating/1080 steel low carbon substrate system, are plotted on the same graph (Figure 5-6) for comparison against applied indentation load $P$.

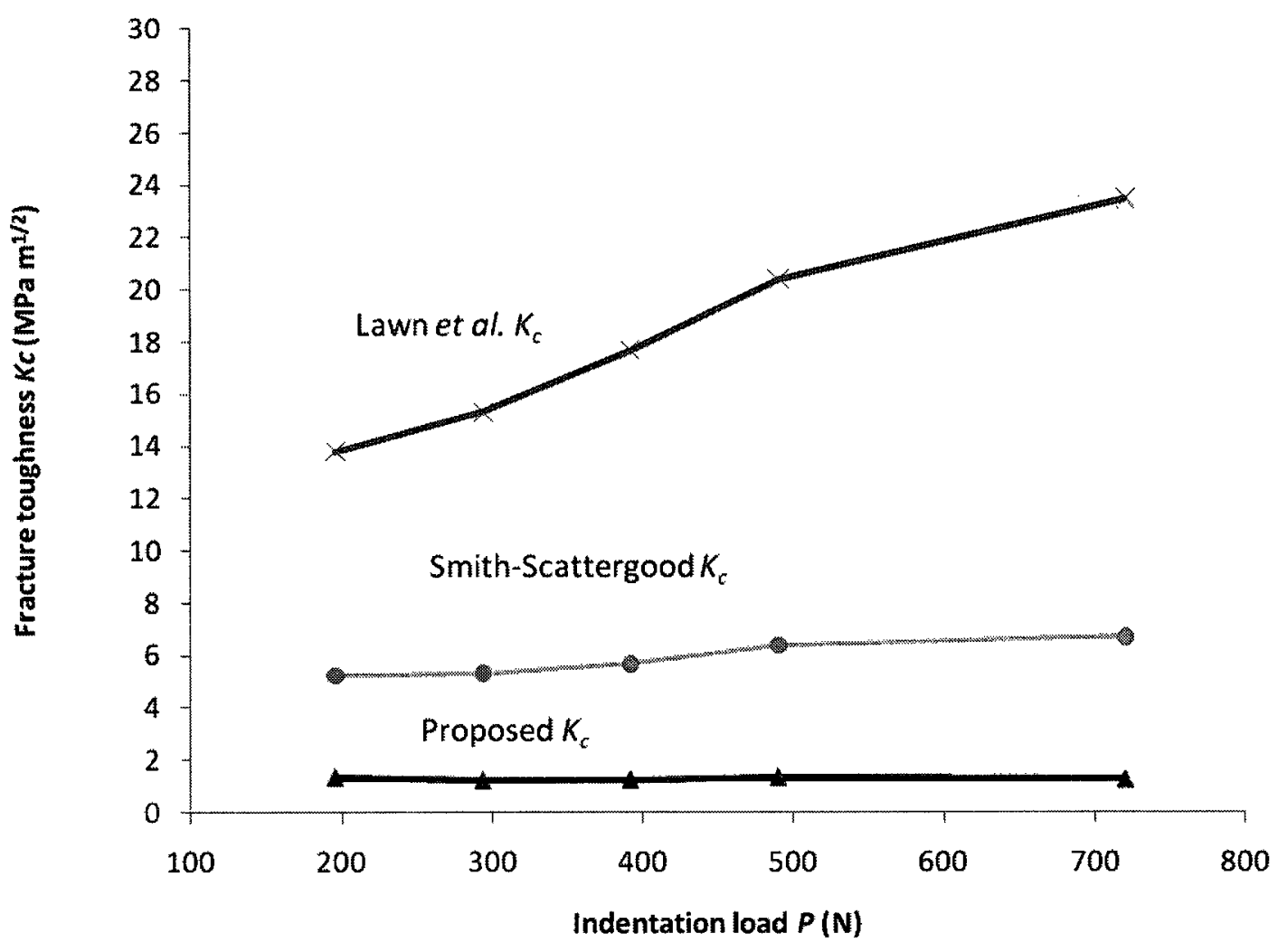

Figure 5-6. Comparison of the fracture toughness values predicted with the proposed model, Smith-Scattergood model and Lawn et al. model.

It is clear from Figure 5-6 that both Smith-Scattergood model and Lawn et al. model predict much higher fracture toughness values for the $\mathrm{WC} / 10 \mathrm{Co} / 4 \mathrm{Cr}$ coating/1080 steel low carbon substrate system than those predicted with the proposed fracture toughness model. It is also evident from Figure 5-6 that the 
predictions made with the proposed model do not vary much with the indentation load. The fluctuations in fracture toughness predictions are within finite bounds forming almost a straight line within the scale of the graph. On the contrary, the predictions made with both Smith-Scattergood model and Lawn et al. model vary or increase with the indentation load. In other words, the proposed model enables a reasonable prediction of fracture toughness for brittle coating/ductile substrate systems, while both Smith-Scattergood model and Lawn et al. model exhibit deficiencies in fracture toughness assessment of these systems. A more detailed discussion about advantages and limitations of the proposed model is provided in subsequent sections as follows.

\subsection{Discussion}

As demonstrated previously, the proposed elastic-plastic fracture mechanics model provides a quantitative basis for fracture toughness analysis of brittle coating/ductile substrate systems with special features. It exhibits many advantages over the existing models, as discussed below.

The proposed approach to fracture toughness assessment is specifically developed for brittle coatings on ductile substrates and can be applied to coatings of any thickness. The salient features of this model can be summarized as: 1) consideration of an elliptical crack profile; 2) incorporation of the substrate material properties in terms of composite modulus $E_{m}$ and composite hardness $H_{m}$, and 3) introduction of an interface correction factor to account for the presence of the coating/substrate interface. These effects are discussed individually in the following sections. 


\subsubsection{Crack Shape Effects}

To account for the crack shape effects, an analytical treatment to semi-elliptical crack profiles is developed using Borodachev's solution [18] for an embedded elliptical crack in an infinite body subjected to a point force load. Here, the "point force" represents the residual plastic component resulting from the expansion of the plastic zone under indentation. Henceforth, the residual stress intensity factor is derived for the well-developed radial/median cracks. The scaling relationship between the indentation load $P$ and a point force that approximates the residual stress driving force acting on the crack is defined as $\pi^{-2} P_{r}=\chi_{r} P$ [21]. The residual factor $\chi_{r}$ is a function of material parameters such as Young's modulus $E_{c}$ and Poisson's ratio $v_{c}$ of the coating, a composite hardness $H_{m}$ and the indenter geometry $\psi$. It can be estimated from Eq. (3-16) based on the known material properties of the system in question and an indenter half-angle $\psi$. Overall, depending on the depth of the radial/median crack in the coating, the residual parameter $\chi_{r}$ is determined mostly by the material properties of the coating/substrate system, rather than just the coating material properties.

Borodachev's solution allows for the incorporation of the crack shape influence factor $\beta$ into the analysis of the radial/median cracks in brittle coating/ductile substrate systems. This crack shape influence factor $\beta$ relates to the crack shape ellipticity factor $k^{2}=1-(a / c)^{2}$. It is a distinct feature of the proposed approach in comparison with the relationship developed by Lawn et al. [11] for monolithic brittle materials. Smith and Scattergood [21] also considered the effect of elliptical crack shape in their studies of bulk soda-lime glass under indentation. To address the crack shape ellipticity, Smith and 
Scattergood used an approximate weight function approach [21], the so-called "0 integral", proposed by Oore and Burns [33]. However, the crack shape correction factor, as defined in Eq. (5-2 b), did not entirely account for the crack shape effect for the cases presented in this work, perhaps because the " 0 integral" solution has limitations in the case of a low aspect ratio $(a / c)$ crack, which is pertinent to the case considered in this research.

\subsubsection{Substrate Material Effects}

In general, analysis of the fracture behavior of brittle coating/ductile substrate systems under indentation is always more involved than that for brittle monolithic materials. When examining thin brittle coatings, the response of coating failure to the indentation load is a synthesized effect of the coating and substrate behavior. This means that the coating material properties will dominate the cracking/failure behavior of the coating for shallow indenter penetration and shallow in-depth cracks, while the substrate presence will prevail for deeper indentation. The latter manifests itself not only through a change in material properties (such as hardness) but also through a change in a crack shape and stress redistribution. As a rule of thumb, when it is desirable to avoid influence of the substrate, the indentation depth should be less than $7 \%$ of the coating thickness $[20,30]$. Otherwise, the presence of the substrate starts affecting the response of the coating/substrate system to the indentation load.

To take the substrate material effect into consideration, the composite hardness is included in the analytical solution. A coating/substrate system reacts as a whole due to the expansion of the plastic zone underneath the indenter at a certain applied indentation load and subsequent crack propagation into the coating closer to the substrate interface. 
Eq. (3-15), proposed by Ichimura et al. and used in the present analysis, is based on the relative volume deformation of a film/substrate system [55]. The composite hardness, represented with Eq. (3-15), depends on an average indentation diagonal length and as such, varies at different indentation loads. This variation contributes to a change in the residual factor $\chi_{r}$ value corresponding to the indentation load. The composite hardness, represented with Eq. (3-15), also confirms a dependence of the residual factor $\chi_{r}$ on the material properties of the coating/substrate system through Eq. (3-16), rather than just the coating material properties.

\subsubsection{Coating/Substrate Interface Effects}

When a crack develops in the coating layer under increasing indentation load and propagates towards the coating/substrate interface, the substrate material would have a progressively strong influence on the crack driving force. To determine the interface correction factor for an elliptical crack near a bi-material interface under an arbitrary loading condition, the stress intensity factor (SIF) behavior of an embedded elliptical crack in the infinite medium under remote tensile loading is analyzed based on the analytical solution provided by Noda et al. [58]. By normalizing the SIF with the solution for an elliptical crack in an infinite body under uniform stress so that the load and crack geometry effects are excluded, the influence of the bi-material interface for a wide range of $h / a=1.05 \sim \infty$ and $\mu_{s} / \mu_{c}=0 \sim \infty$, is condensed into an explicit interface correction factor. This is described with Eq. (3-24) for the deepest crack front point, i.e., the point closest to the interface. Then, it is further extrapolated to an entire crack front $(0<\varphi<\pi)$, i.e., Eq. (3-25). To reflect the representation of the interface correction factor, this parameter is fitted to the numerical data of Noda et al. for comparison. In 
contrast to the interface correction factor provided by Noda et al., this new interface correction factor has the following two improvements:

I. It represents directly the interface effect related to the distance of the crack front to the interface.

II. It yields great convenience for engineering analysis by avoiding the cumbersome numerical procedure which is necessarily taken in calculating the interface correction factor by Noda et al.

By normalization, it has been demonstrated earlier that the proposed interface correction factor is independent of loading conditions and can be utilized for an elliptical crack embedded in a bi-material medium under a point load which is pertinent to the case of a sharp (Vickers) indenter considered in the present study. Incorporation of the interface correction factor has significant implications in the analysis of crack propagation in brittle coating/ductile substrate systems. Provided that shear modulus is proportional to Young's modulus through the expression $\mu \propto E$ [65] (where $\mu$ is shear modulus and $E$ is Young's modulus), one can infer from Eq. (3-25) that when the substrate is softer (having a lower Young's modulus) than the coating material, the interface tends to raise the SIF of the approaching median crack, thus pulling the crack to propagate toward the interface, which was, indeed, true for the present case of $\mathrm{WC} / 10 \mathrm{Co} / 4 \mathrm{Cr}$ coating/1080 steel substrate. On the contrary, if the substrate is stiffer (having a higher Young's modulus) than the coating material, the interface will lower the SIF of the approaching crack, thus retarding the crack propagation towards the interface. Alternatively, if the crack driving force is greater than the fracture toughness of the substrate material, the crack may propagate into the substrate. Otherwise, a crack will be 
confined in the coating layer. In the present case, the fracture toughness of the substrate material (1080 low carbon steel) is about $75 \mathrm{MPa}^{1 / 2}$ [66], which is much higher than the coating fracture toughness estimated in this study. Therefore, the radial/median cracks, observed in the indentation tests, were all confined in the coating layer. In this regard, other existing models such as Lawn et al. model [11] and Smith-Scattergood model [21] have substantial limitations.

\subsubsection{Prediction of Fracture Toughness and Fracture Behaviour}

Using the proposed approach to fracture toughness assessment of brittle coating/ductile substrate systems, a value of fracture toughness $K_{c}=1.29 \pm 0.05 \mathrm{MPa} \mathrm{m}^{1 / 2}$ was obtained for the $\mathrm{WC} / 10 \mathrm{Co} / 4 \mathrm{Cr}$ coating/1020 steel substrate system. This value was found reasonable (within 19\% error) in comparison with the already known fracture toughness value of $K_{c}=1.6 \pm 0.9 \mathrm{MPa} \mathrm{m}^{1 / 2}$ for the similar system of WC/12Co coating/1020 steel substrate system, estimated by Lima et al. [38]. In other words, the fracture toughness of $1.29 \pm 0.05 \mathrm{MPa} \mathrm{m} \mathrm{m}^{1 / 2}$ calculated with the proposed model is a realistic value for the $\mathrm{WC} / 10 \mathrm{Co} / 4 \mathrm{Cr}$ coating/1080 steel substrate system, as it is within the same order of magnitude with Lima et al. value of $1.6 \pm 0.9 \mathrm{MPa} \mathrm{m} \mathrm{m}^{1 / 2}$. Moreover, the predictions of fracture toughness made with the proposed model are scattered within a small finite range (i.e., $\pm 4 \%$ ) around the average value of the fracture toughness of $K_{c}=1.29 \mathrm{MPa} \mathrm{m}^{1 / 2}$ for the $\mathrm{WC} / 10 \mathrm{Co} / 4 \mathrm{Cr}$ coating/1080 steel substrate system. This also verifies the reliability of the proposed model in terms of accuracy of the fracture toughness prediction.

It was also demonstrated that the experimentally observed radial crack length exhibited an apparent non-linear behavior as indentation load increased. Such behavior 
can be attributed to the effect of the crack shape deviating from the ideal penny-shape geometry over the entire growth path. The proposed model, in terms of Eq. $(5-1 \mathrm{c})$, incorporates a crack shape influence factor $\beta$ and the residual factor $\chi_{r}$ into the analysis. These factors vary with the indentation load, while the fracture toughness $K_{c}$ remains invariable being a coating/substrate material parameter. As a result, it was found that the proposed fracture toughness model was sensitive to a non-linear change in radial crack behavior with the indentation load (i.e., it would predict a variable slope along the curve of the radial crack length $c^{3 / 2}$ vs. the indentation load $P$ ). Hence, the proposed model can interpret well the experimental observations or data with regards to the non-linear relationship between the radial crack length $c^{3 / 2}$ vs. the indentation load $P$.

Furthermore, the fracture toughness predicted with one of the existing fracture toughness models by Smith and Scattergood [21] provides a different estimate of $K_{c}=5.965 \pm 0.735 \mathrm{MPa} \mathrm{m}^{1 / 2}$ over a wide load range $(196 \leq P \leq 720 \mathrm{~N})$ for the $\mathrm{WC} / 10 \mathrm{Co} / 4 \mathrm{Cr}$ coating/1080 steel substrate system. Smith-Scattergood model over predicts the fracture toughness by at least 3 times in comparison with Lima et al. fracture toughness value of $K_{c}=1.6 \mathrm{MPa} \mathrm{m} \mathrm{m}^{1 / 2}$ for the $\mathrm{WC} / 12 \mathrm{Co}$ coating/1020 steel substrate system. Moreover, in comparison with the fracture toughness estimated with the proposed model (i.e., 1.29 MPa m ${ }^{1 / 2}$ ), the prediction made with Smith-Scattergood model is higher by 4 times. Smith-Scattergood model fracture toughness values are widely scattered. This exposes a deficiency of Smith-Scattergood model in predicting the fracture toughness for brittle coating/ductile substrate systems. In the case of Lawn et al. model, the fracture toughness is predicted as $K_{c}=18.7 \pm 4.85 \mathrm{MPa} \mathrm{m}^{1 / 2}$, which over predicts the fracture toughness for the $\mathrm{WC} / 10 \mathrm{Co} / 4 \mathrm{Cr}$ coating/1080 steel substrate system 
by 11 times in comparison with the value of $K_{c}=1.29 \mathrm{MPa} \mathrm{m}^{1 / 2}$ determined with the proposed model. The fracture toughness predictions made with Lawn et al. model are also largely scattered. This indicates the inability of Lawn et al. model in predicting accurately the fracture toughness for brittle coating/ductile substrate systems.

In conclusion, it is evident that the fracture toughness values predicted with both Lawn et al. model and Smith-Scattergood model vary with an increasing indentation load. Hence, these models could not really predict an average value for the fracture toughness of brittle coating/ductile substrate systems. Provided that fracture toughness is a material or coating/substrate system parameter, which is independent of load, the reliability of predictions made with both Lawn et al. model and Smith-Scattergood model is doubtful. In this regard, the developed model from the present research has made significant improvement in comparison with the existing fracture toughness models for brittle coating/ductile substrate systems.

\subsubsection{Implications for the Coating System Design}

Two main outcomes have been achieved from the proposed fracture toughness model that may have significant implications on the coating system design. Firstly, when a coating is deposited on a ductile substrate with significantly higher fracture toughness, in most cases, the substrate would arrest the median crack propagation (provided that the interfacial adherence is good). Secondly, the ductile substrate seems to have a "holding" effect on the radial crack propagation such that it results in a shorter crack length than would be predicted by Lawn et al. theory. Therefore, it is reasonable to believe that formation of elliptical radial/median cracks is a dominant fracture mode in brittle coating/ductile substrate systems under indentation, if debonding does not occur. 
Moreover, the crack depth can be approximated by the average coating thickness which yields a great convenience in terms of engineering applications. Hence, Eq. (3-10 a) can be used as a convenient tool to analyze the fracture behavior of brittle coating/ductile substrate systems. However, a drawback of this approach, similarly to the treatment of monolithic brittle materials provided by Lawn et al. [11], is that the residual factor $\chi_{r}$ has to be estimated based on a coating/substrate system with known material properties.

Another prospective departure from the idealized situation may occur regarding the size of the residual field. In the present work the author follows an approach that is well-established in indentation mechanics $[1,5]$; as such, the residual field is treated as being far from the actual elliptical crack profile (crack front). Hence, the residual field can be approximated as a point that acts as a driving force on the crack. Consequently, the fracture toughness relationship for the elliptical crack under the centralized point force load can be utilized in the analysis. The radial cracks in the $\mathrm{WC} / 10 \mathrm{Co} / 4 \mathrm{Cr}$ coating/1080 low carbon steel system, that are investigated in this research, are much longer than the coating thickness and as such could be regarded as well-developed elliptical cracks under a point force load. In practice, there could be an obvious departure from this assumption, especially for brittle thin coatings under small loads containing short cracks. In such circumstances, the residual field may be of comparable size to a well-developed radial/median crack. Investigation of significance of this phenomenon on the proposed fracture toughness relationship is a subject of future work.

In summary, an improved approach to the fracture toughness assessment of brittle coating/ductile substrate systems is proposed in the present research. This approach has 
been verified by experiment and analysis on the $\mathrm{WC} / 10 \mathrm{Co} / 4 \mathrm{Cr}$ coating/1080 low carbon steel substrate system. The distinct features of the proposed model are incorporation of i) the crack shape effect, ii) the substrate material influence, and iii) the coating/substrate interface correction factor. Overall, the proposed approach to the fracture toughness assessment of brittle coating/ductile substrate systems has been proven to have a distinct advantage over other fracture toughness models such as by Lawn et al. [11] and Smith and Scattergood [21], which are presently utilized for the fracture toughness assessment of coating/substrate systems. 


\section{Chapter 6. Conclusions and Future Work}

\subsection{Conclusions}

In the present study, an analytical fracture toughness model was developed for brittle coating/ductile substrate systems, and a series of indentation tests were performed on a $\mathrm{WC} / 10 \mathrm{Co} / 4 \mathrm{Cr}$ coating/ 1080 low carbon steel substrate system for validation of the model. The following conclusions can be drawn from the analytical and experimental results as well as from the model verification and discussion:

1. The radial/median cracks developing in brittle coating/ductile substrate systems under indentation are most likely to form in elliptical shape with the coating thickness as the upper limit of the crack depth, when the substrate material has significantly higher fracture toughness than the coating material.

2. The proposed model provides a sound estimate of the fracture toughness of the $\mathrm{WC} / 10 \mathrm{Co} / 4 \mathrm{Cr}$ coating/1080 low carbon steel substrate system.

3. The well-developed radial/median crack exhibits distinguishably non-linear dependency in terms of the relation between the radial crack length $c^{3 / 2}$ and the applied indentation load $P$.

4. The substrate material has a strong influence on the coating fracture behavior of the coating/substrate system.

5. A proposed analytical model developed in this research incorporates three distinct features, which improve Lawn et al. original formulation:

I. An elliptical crack shape influence factor $\beta$ introduced by adopting Borodachev's solution for an elliptical crack under a concentrated force. 
II. An improved interface correction factor, derived from analyzing the results of Noda et al. for an elliptical crack near a bi-material interface, which expresses explicitly its dependence on the $h / a$ ratio and shear moduli ratio $\mu_{s} / \mu_{c}$;

III. A composite hardness formula that takes into account the influence of the substrate material properties on the coating/substrate system hardness.

\subsection{Summary and Future Work}

The considerations in the present model for the fracture toughness assessment of brittle coating/ductile substrate systems would have eliminated the previous uncertainties regarding the influence of the coating/substrate interface and the substrate material associated with the indentation depth exceeding $7 \%$ of the coating thickness [5]. It has been shown that the proposed model predicts well the fracture toughness of the brittle coating/ductile substrate system ( $\mathrm{WC} / 10 \mathrm{Co} / 4 \mathrm{Cr}$ coating/1080 low carbon steel substrate) in question and is also capable of predicting the non-linear crack propagation behavior. Based on the experimental observation in the present study, the proposed model predicts a fracture toughness value of $1.29 \mathrm{MPa} \mathrm{m}^{1 / 2}$ for the $\mathrm{WC} / 10 \mathrm{Co} / 4 \mathrm{Cr}$ coating/1080 steel substrate, which is very close to the value of $1.6 \mathrm{MPa} \mathrm{m}^{1 / 2}$ for the similar WC/12Co coating/1020 steel substrate system, obtained by Lima et al. [38].

Since the key parameters included in the proposed model are the parameters that may be determined by means of the well-established indentation technique, it imposes no limitations on the type of the system in question. As it stands, the proposed model can be directly applied to analysis of the fracture toughness and fracture behavior of brittle coating/ductile substrate systems with indentation tests, similar to Lawn et al. model developed for monolithic brittle materials. 
To further improve this model and clarify the uncertainties, the scope of the future work should include testing of this approach against brittle coating/ductile substrate systems with different coating thicknesses and different material properties. It is also advisable to test the coating/substrate systems with the radial/median cracks of various depths to fully verify the range of the proposed approach. The necessary modifications to the analysis can be made to develop similar fracture mechanics models for the brittle coating/ductile substrate systems containing well-developed radial/median crack induced by either Berkovich or cube indenters. 


\section{References}

[1] R. Lima, B. Marple, Thermal Spray Coatings Engineered from Nanostructured Ceramic Agglomerated Powders for Structural, Thermal Barrier and Biomedical Applications: A review, JTST, Vol. 16, Issue 1, 2007, pp. 40-67

[2] J. DeMasi-Marcin, D. Gupta, Protective Coatings in the Gas Turbine Engine, Surf. Coat. Technol., 68/69 (1994) 1-9

[3] Y. Rhee, H. Kim, Y. Deng, B. Lawn, Contact-induced Damage in Ceramic Coatings on Compliant Substrates: Fracture Mechanics and Design, J. Am. Ceram. Soc. 84 [5] 1066-72 (2001)

[4] B. Lawn, A. Pajares, P. Miranda, Y. Deng, Ceramic-based Layer Structures for Biomechanical Applications, EMSAT, pp. 1-8

[5] S. Zhang, D. Sun, Y. Fu, H. Du, Toughness Measurement of Thin Films: A Critical Review, Surf. Coat. Technol. 198 (2005) 74-84

[6] I. Campos, R. Rosas, U. Figueroa, C. Velazquez, A. Meneses, A. Guevara, Fracture Toughness Evaluation using Palmqvist Crack Models on AISI 1045 Borided Steels, Mater. Sci. Eng., A 488 (2008) 562-568

[7] J. Chen, S. Bull, Indentation Fracture and Toughness Assessment for Thin Optical Coatings on Glass, J. Phys. D: Appl. Phys. 40 (18), 5401-5417

[8] D. Shetty, I. Wright, N. Mincer, A. Clauer, Indentation Fracture of WC/Co Cermets, J. Mater. Sci. 20 (1985) 1873-1882

[9] D. Marshall, B. Lawn, An Indentation Technique for Measuring Stresses in Tempered Glass Surfaces, J. Am. Ceram. Soc. - Discussions and Notes, Vol. 60, No. 1-2, pp. 86-87

[10] K. Niihara, A Fracture Mechanics Analysis of Indentation-induced Palmqvist Crack in Ceramics, J. Mater. Sci. Lett. 2 (1983) 221-223

[11] B. Lawn, A. Evans, D. Marshall, Elastic/plastic Indentation Damage in Ceramics: The Median/Radial Crack System, J. Am. Ceram. Soc. 63 (1980) 574

[12] B. Beake, M. Garcia, J. Smith, Micro-impact Testing: A New Technique for Investigating Fracture Toughness, Thin Solid Films 398-399 (2001) pp. 438-443

[13 ] A. Fischer-Cripps, B. Lawn, A. Pajares, and L. Wei, Stress Analysis of Elastic-plastic Contact Damage in Ceramic Coatings on Metal Substrates, J. Am. Ceram. Soc., 79 (10) (1996) pp. 2619-25 
[14] H. Chai and B. Lawn, Cracking in Brittle Laminates from Concentrated Loads, Acta Mater., 50 (2002) pp. 2613-2625

[15] D. Marshall, B. Lawn, and A. Evans, Elastic/plastic Indentation Damage in Ceramics: The Lateral Crack System, J. Am. Ceram. Soc., Vol. 65, No. 11, pp. 561-566

[16] A. Fischer-Cripps and B. Lawn, Stress Analysis of Contact Deformation in Quasi-plastic Ceramics, J. Am. Ceram. Soc., 79 [10] (1996) pp. 2609-18

[17] Y. Rhee, H. Kim, Y. Deng, and B. Lawn, Brittle Fracture versus Quasi Plasticity in Ceramics: A Simple Predictive Index, J. Am. Ceram. Soc., 84 [3] (2001) pp. 561-566

[18] N. Borodachev, An Elliptical Crack under Point Forces Applied to its Surfaces, J. Appl. Math. Mech., Vol. 64, No. 3, pp. 477-483

[19] X. Lambert, G. Glinka, Approximate Weight Functions for Embedded Elliptical Cracks, Eng. Fract. Mech., Vol. 59, No. 3, pp. 381-392, 1998

[20] E. Puchi-Cabrera, A New Model for the Computation of the Composite Hardness of Coated Systems, Surf. Coat. Technol., 160 (2002) 177-186

[21] S. Smith, R. Scattergood, Crack-shape Effects for Indentation Fracture Toughness Measurements, J. Am. Ceram. Soc., 75 [2] 305-15 (1992)

[22] T. Hiroshi, P. Paris, G. Irwin (February 2000) The Stress Analysis of Cracks Handbook, $A S M E$

[23] R. Hill, The Mathematical Theory of Plasticity, Oxford University Press Inc., New York, USA, 1950

[24] G. Anstis, P. Chantikul, B. Lawn, D. Marshall, A Critical Evaluation of Indentation Techniques for Measuring Fracture Toughness: I, Direct Crack Measurements, J. Am. Ceram. Soc., Vol. 64, No. 9, pp. 533-538

[25] H. Li, K. Khor, P. Cheang, Young's Modulus and Fracture Toughness Determination of High Velocity Oxy-Fuel-Sprayed Bioceramic Coatings, Surf. Coat. Technol. 155 (2002) 21-32

[26] P. Kodali, K. Walter, M. Nastasi, Investigation of Mechanical and Tribological Properties of Amorphous Diamond-like Carbon Coatings, Tribol. Int. Vol. 30, No. 8, pp. 591-598, 1997

[27] J. Mecholsky, Y. Tsai, W. Drawl, Fracture Studies of Diamond Films on Silicon, J. Appl. Phys. 1992, 71, 4875-4881 
[28] P. Jedrzejowski, J. Klemberg-Sapieha, L. Martinu, Relationship Between the Mechanical Properties and the Microstructure of Nanocomposite TiN/SiN ${ }_{1.3}$ Coatings Prepared by Low Temperature Plasma Enhanced Chemical Vapor Deposition, Thin Solid Films 426 (2003) 150-159

[29] X. Li, B. Bhushan, Micro/nanomechanical Characterization of Ceramic Films for Microdevices, Thin Solid Films 340 (1999) 210-217

[30] J. Malzbender, G. de With, J. den Toonder, Elastic Modulus, Indentation Pressure and Fracture Toughness of Hybrid Coatings on Glass, Thin Solid Films 366 (2000) 139-149

[31] J. Malzbender, J. den Toonder, A. Balkenende, G. de With, Measuring Mechanical Properties of Coatings: A Methodology Applied to Nano-particle-filled Sol-gel Coatings on Glass, Mater. Sci. Eng., R 36 (2002) 47-103

[32] J. Malzbender, G. de With, Elastic Modulus, Hardness and Fracture Toughness of $\mathrm{SiO}_{2}$-filled Methyltrimethoxysilane Coatings on Glass Substrates, J. Non-Cryst. Solids, 265 (2000) 51-60

[33] M. Oore, D. Burns, Estimation of Stress Intensity Factors for Embedded Irregular Cracks Subjected to Arbitrary Normal Stress Fields, J. Pressure Vessel Technol., 102, 202-11 (1980)

[34] X. Wang, S. Lambert, Stress Intensity Factors for Low Aspect Ratio Semi-Elliptical Surface Cracks in Finite-Thickness Plates Subjected to Non-uniform Stresses, Eng. Fract. Mech. Vol. 51, No. 4, pp. 517-532, 1995

[35] X. Wang, S. Lambert, Stress Intensity Factors and Weight Functions for High Aspect Ratio Semi-Elliptical Surface Cracks in Finite-Thickness Plates, Eng. Fract. Mech. Vol. 57, No. 1, pp. 13-24, 1997

[36] A. Evans, T. Wilshaw, Quasi-static Solid Particle Damage in Brittle Solids I. Observations. Analysis and Implications, Acta Metall. 24 (1976) 939

[37] K. Niihara, R. Morena, D. Hasselman, Evaluation of $\mathrm{K}_{\mathrm{Ic}}$ of Brittle Solids by the Indentation Method with Low Crack-to-indent Ratios, J. Mater. Sci. Letters 1 (1982) 13-16

[38] M. Lima, C. Godoy, P. Modenesi, J. Avelar-Batista, A. Davison, A. Matthews, Coating Fracture Toughness Determined by Vickers Indentation: An Important Parameter in Cavitation Erosion Resistance of WC/Co Thermally Sprayed Coatings, Surf. Coat. Technol. 177-178 (2004) 489-496

[ 39 ] A. Evans, E. Charles, Fracture Toughness Determination by Indentation, J. Am. Ceram. Soc., 59 [7-8] 371-72 (1976) 
[ 40 ] K. Niihara, R. Morena, D. Hasselman, Further Reply to "Comment on 'Elastic/Plastic Indentation Damage in Ceramics: The Median/Radial Crack System"', Commun. Am. Ceram. Soc., July 1982, C-116

[41] J. Lankford, Indentation Microfracture in the Palmqvist Crack Regime: Implications for Fracture Toughness Evaluation by the Indentation Method, J. Mater. Sci. Lett. 1 (1982) 493-495

[42] A. Roman, D. Chicot, J. Lesage, Indentation Test to Determine the Fracture Toughness of Nickel Phosphorus Coatings, Surf. Coat. Technol., 155 (2002) 161-168

[43] X. Li, D. Diao, B. Bhushan, Fracture Mechanisms of Thin Amorphous Carbon Films in Nanoindnentation, Acta Mater., (1997) 45, 4453-4461

[44] S. Neralla, D. Kumar, S. Yarmolenko, J. Sankar, Mechanical Properties of Nanocomposite Metal-ceramic Thin Films, Compos. Eng., Vol. 35, Issue 2, March 2004, pp. 157-162

[45] X. Li, B. Bhushan, Evaluation of Fracture Toughness of Ultra-thin Amorphous Carbon Coatings Deposited by Different Deposition Techniques, Thin Solid Films 355-356 (1999) 330-336

[46] J. Ding, Y. Meng, S. Wen, Mechanical Properties and Fracture Toughness of Multilayer Hard Coatings Using Nanoindentation, Thin Solid Films 371 (2000) 178

[ 47 ] A. Evans, E. Charles, Fracture Toughness Determinations by Indentation, J. Am. Ceram. Soc, Vol. 59, No. 7-8, p. 371

[48] Z. Jiang, F. Lu, W. Tang, S. Wang, Y. Tong, T. Huang, J. Liu, Accurate Measurement of Fracture Toughness of Free Standing Diamond Films by Three-point Bending Tests with Sharp Pre-cracked Specimens, Diamond Relat. Mater. 9 (2000) 1734-1738

[49] B. Cotterell, Z. Chen, Buckling and Cracking of Thin Films on Compliant Substrates under Compression, Int. J. Fract. 104:169-179, 2000

[50] S. Britvee, The Stability of Elastic Systems, Pergamon Press, New York, 1973

[51] K. Holmberg, A. Laukkanen, H. Ronkainen, K. Wallin, S. Varjus, A Model for Stresses, Crack Generation and Fracture Toughness Calculation in Scratched TiN-coated Steel Surfaces, Wear 254 (2003) 278-291

[52] U. Wiklund, M. Bromark, M. Larsson, P. Hedenquist, S. Hogmark, Cracking Resistance of Thin Hard Coatings Estimated by Four-point Bending, Surf. Coat. Technol. 91 (1997) 57-63 
[53] A. Laukkanen, K. Holmberg, J. Korkinen, H. Ronkainen, K. Wallin, S. Varjus, Tribological Contact Analysis of a Rigid Ball Sliding on a Hard Coated Surface, Part III: Fracture Toughness Calculation and Influence of Residual Stresses, Surf. Coat. Technol., $\mathrm{xx}$ (2005) $\mathrm{xxx}-\mathrm{xxx}$. Available online at www.sciencedirect.com

[54] K. Holmberg, A. Laukkanen, H. Ronkainen, K. Wallin, Tribological Analysis of Fracture Conditions in Thin Surface Coatings by 3D FEM Modeling and Stress Simulations, Tribol. Int. 38 (2005) 1035-1049

[55] H. Ichimura, F. Rodriguez, A. Rodrigo, The Composite and Film Hardness of TiN Coatings Prepared by Cathodic Arc Evaporation, Surf. Coat. Technol. 127 (2000) 138

[56] J. Newman, Jr., "Fracture Mechanics Parameters for Small Fatigue Cracks," in Small-Crack Test Methods, ASTM STP 1149, J. Larson and J. E. Allison, Eds. Philadelphia: American Society for Testing and Materials, 1992, pp. 6-33.

[57] B. Nuller, E. Karapetian, M. Kachanov, On the Stress Intensity Factor for the Elliptical Crack, Int. J. Fract. 92:L17-L20, 1998

[58] N. Noda, T. Kouyama, Y. Kinoshita, Stress Intensity Factors for an Inclined Elliptical Crack Near a Bimaterial Interface, Eng. Fract. Mech. 73 (2006) 1292-1320

[59] H. Tada, P. Paris, G. Irwin (2000). The Stress Analysis of Cracks Handbook (3 ed.). American Society of Mechanical Engineers

[60] A. Buang, Master thesis, Cracking and Fracture Analysis of HVOF Coatings under Vickers Indentation, Carleton University, Canada, 2008

[61] M. Birkholz, Thin Film Analysis by X-Ray Scattering, ISBN:3-527-31052-5, Hardcover, 378 pages, February 2006

[62] E 384-08a, Standard Test Method for Micro-indentation Hardness of Materials, ASTM International, May 23, 2008

[63] http://www.ultrahardmaterials.co.uk/CeTZPvsSteels.html. Last visited, April 2010

[64] http://www.fibics.com/fib/tutorials/introduction-focused-ion-beam-systems/4/

[65] H. McSkimin, P. Andreatch, Elastic Moduli of Diamond as a Function of Pressure and Temperature, J. Appl. Phys., 43, 2944-2948 (1972)

[66] H. Roy, N. Parida, S. Sivaprasad, S. Tarafder, K. Ray, Acoustic Emissions During Fracture Toughness Tests of Steels Exhibiting Varying Ductility, Mater. Sci. Eng. A 486 (2008) 562-571 